\title{
MECHANISMS LEADING TO CO-EXISTENCE OF GAS HYDRATE IN OCEAN SEDIMENTS
}

\author{
Final Report
}

1 October 2006 to 31 December 2011

\author{
by \\ Steven Bryant \\ and \\ Ruben Juanes \\ 31 March 2012
}

Work Performed under Contract No. DE-FC26-06NT43067

Prepared for U.S. Department of Energy

Robert Vagnetti, Project Manager

\author{
U.S. Department of Energy \\ National Energy Technology Laboratory \\ P.O. Box 880 \\ Morgantown, WV 26507-0880
}

Prepared by

Center for Petroleum and Geosystems Engineering

The University of Texas at Austin

Austin, Texas 78712

and

Department of Civil and Environmental Engineering

MIT

Cambridge, Massachusetts 02139 


\section{DISCLAIMER}

This report was prepared as an account of work sponsored by an agency of the United States Government. Neither the United States Government nor any agency thereof, nor any of their employees, makes any warrantee, express or implied, or assumes any legal liability or responsibility for the accuracy, completeness, or usefulness of any information, apparatus, product, or process disclosed, or represents that its use would not infringe privately owned rights. Reference herein to any specific commercial product, process, or service by trade name, trademark, manufacturer, or otherwise does not necessarily constitute or imply its endorsement, recommendation, or favoring by the United States Government or any agency thereof. The views and opinions of authors expressed herein to not necessarily state or reflect those of the United States Government or any agency thereof. 


\begin{abstract}
In this project we have sought to explain the co-existence of gas and hydrate phases in sediments within the gas hydrate stability zone. We have focused on the gas/brine interface at the scale of individual grains in the sediment. The capillary forces associated with a gas/brine interface play a dominant role in many processes that occur in the pores of sediments and sedimentary rocks. The mechanical forces associated with the same interface can lead to fracture initiation and propagation in hydrate-bearing sediments. Thus the unifying theme of the research reported here is that pore scale phenomena are key to understanding large scale phenomena in hydrate-bearing sediments whenever a free gas phase is present.

Our analysis of pore-scale phenomena in this project has delineated three regimes that govern processes in which the gas phase pressure is increasing: fracturing, capillary fingering and viscous fingering. These regimes are characterized by different morphology of the region invaded by the gas. On the other hand when the gas phase pressure is decreasing, the corresponding regimes are capillary fingering and compaction. In this project, we studied all these regimes except compaction. Many processes of interest in hydrate-bearing sediments can be better understood when placed in the context of the appropriate regime. For example, hydrate formation in sub-permafrost sediments falls in the capillary fingering regime, whereas gas invasion into ocean sediments is likely to fall into the fracturing regime.

Our research provides insight into the mechanisms by which gas reservoirs are converted to hydrate as the base of the gas hydrate stability zone descends through the reservoir. If the reservoir was no longer being charged, then variation in grain size distribution within the reservoir explain hydrate saturation profiles such as that at. Elbert, where sand-rich intervals containing little hydrate are interspersed between intervals containing large hydrate saturations. Large volumes (of order one pore volume) of gaseous and aqueous phases must be transported into the gas hydrate stability zone. The driver for this transport is the pressure sink induced by a reduction in occupied pore volume that accompanies the formation of hydrate from gas and water. Pore-scale imbibition models and bed-scale multiphase flow models indicate that the rate-limiting step in converting gas to hydrate is the supply of water to the hydrate stability zone. Moreover, the water supply rate is controlled by capillarity-driven flux for conditions typical of the Alaska North Slope. A meter-scale laboratory experiment confirms that significant volumes of fluid phases move into the hydrate stability zone and that capillarity is essential for the water flux. The model shows that without capillarity-driven flux, large saturations of hydrate cannot form. The observations of thick zones of large saturation at Mallik and Mt Elbert thus suggest that the primary control on these systems is the rate of transport of gaseous and aqueous phases, driven by the pressure sink at the base of the gas hydrate stability zone.

A key finding of our project is the elucidation of "capillary fracturing" as a dominant gas transport mechanism in low-permeability media. We initially investigate this phenomenon by means of grain-scale simulations in which we extended a discrete element mechanics code (PFC, by Itasca) to incorporate the dynamics of first singlephase and then multiphase flow. A reductionist model on a square lattice allows us to determine some of the fundamental dependencies of the mode of gas invasion (capillary
\end{abstract}


fingering, viscous fingering, and fracturing) on the parameters of the system. We then show that the morphology of the gas-invaded region exerts a fundamental control on the fabric of methane hydrate formation, and on the overpressures caused by methane hydrate dissociation. We demonstrate the existence of the different invasion regimes by means of controlled laboratory experiments in a radial cell. We collapse the behavior in the form of a phase diagram fully characterized by two dimensionless groups: a modified capillary number and a "fracturing number" that reflects the balance between the pressure forces that act to open conduits in the granular pack, and frictional forces that resist it. We use all this small-scale knowledge to propose simple mechanistic models of gas migration and hydrate formation at the geologic bed scale. We propose that methane transport in lake and oceanic sediments is controlled by dynamic conduits, which dilate and release gas as the falling hydrostatic pressure reduces the effective stress below the tensile strength of the sediments. We test our model against a four-month record of hydrostatic load and methane flux in Upper Mystic Lake, Mass., USA, and show that it captures the complex episodicity of methane ebullition. Our quantitative conceptualization opens the door to integrated modeling of methane transport to constrain global methane release from lakes and other methane-rich sediment systems, and to assess its climate feedbacks. 


\section{TABLE OF CONTENTS}

DISCLAIMER

Page

ABSTRACT.

TABLE OF CONTENTS.

iii

EXECUTIVE SUMMARY

Unifying Theme

Key Findings

Development and Application of Bed Scale Models.....

Capillarity Dominated Behavior.

Geologic Context for Capillarity-Dominated Processes in Sub-Permafrost

Hydrate-Bearing Sediments

Physical Phenomena Critical to the Proposed Model

Gas reservoir Conversion to Hydrate: Bed-Scale Volumetric

Considerations

Gas Reservoir Conversion to Hydrate: Role of Pore-Scale Imbibition

Summary of Influence of Pore-Scale Imbibition on Hydrate Saturation Profiles

Gas Reservoir Conversion to Hydrate: Role of Pressure-Driven Fluid

Phase Transport at Bed-Scale

Summary of Applicability of Pressure-Driven Transport Model for Conversion of Gas Reservoirs to Hydrate

Gas Reservoir Conversion to Hydrate: Role of Capillarity-Driven Fluid Phase Transport at Bed-Scale

Summary of Applicability of Capillarity-Driven Transport Model for Conversion of Gas Reservoirs to Hydrate

Gas Reservoir Conversion to Hydrate: Experimental Validation of Fluid Transport in Response to Hydrate Formation

References for Capillarity Dominated Behavior in Hydrate-Bearing Sediments

Fracturing Dominated Behavior

Preferential mode of gas invasion in sediments: Grain-scale mechanistic model of coupled multiphase fluid flow and sediment mechanics

Crossover from fingering to fracturing in deformable disordered media ......

Thermodynamic and hydrodynamic constraints on overpressure caused by hydrate dissociation: A pore-scale model ..............................................

Capillary fracturing in granular media.

A conduit dilation model of methane venting from lake sediments

Numerical modeling of hydrate formation and methane gas transport through dynamic conduits

References for Fracturing Dominated Behavior.....

Appendix - Description of Underlying Models.................................................. 222

Model Sediments

Advanced Methods for Simulating Drainage and Imbibition 
Coupled Capillarity-Controlled Fluid Displacement with Mechanics.......... 223

Publications on Hydrate-Bearing Sediments ............................................. 223

Theses and Dissertations .......................................................................... 224

Progress Reports ............................................................................ 224 


\section{EXECUTIVE SUMMARY}

\section{Unifying Theme}

A remarkable feature of many naturally occurring gas hydrates is the presence of a free gas phase migrating through sediment that also contains stable hydrate. The long term persistence of these features defies expectations based on thermodynamics. In this project we have sought to explain the co-existence of gas and hydrate phases by focusing on the gas/brine interface. This interface is present when a gas phase occupies any part of a sediment, from the gas/water contact at the base of a free gas accumulation to the meniscus at the leading edge of a fracture induced by gas pressure. The capillary forces associated with a gas/brine interface play a dominant role in many processes that occur in the pores of sediments and sedimentary rocks. Thus the unifying theme of the research reported here is that pore scale phenomena are key to understanding large scale phenomena in hydrate-bearing sediments whenever a free gas phase is present. Pore scale phenomena of particular interest include drainage, imbibition, grain displacement, and growth/breakage of hydrate skins on gas/water interfaces. Large scale phenomena notably include the spatial distribution of hydrate saturation, especially when hydrate formation involves a free gas phase, and the morphology of hydrate, from lenses and filled fractures to veins and filled pores.

We developed a set of new models of relevant pore scale phenomena in this project, and these have been described in progress reports and a series of papers. This report focuses not on these models but on their application at the bed-scale to certain processes in hydrate-bearing sediments. One application is to sub-permafrost accumulations of hydrate, created as the base of the gas hydrate stability zone descended through previously established gas reservoirs. Another application is to sediments in deep water into which gas invasion occurs. The results provide useful insight into the factors governing the bed-scale behavior.

A major scientific contribution of this project is our identification of two broad regimes for gas displacing brine within sediments: a fracturing regime, which applies for low permeability sediments, and a fingering regime, which applies in higher permeability sediments. The fingering regime is subdivided into capillary fingering (classical invasion percolation) at small flow rates and viscous fingering at large flow rates. Many processes of interest in hydratebearing sediments can be better understood when placed in the context of the appropriate regime. For example, hydrate formation in sub-permafrost sediments falls in the capillary fingering regime, whereas gas invasion into ocean sediments is likely to fall into the fracturing regime.

The capillarity-controlled regime for sub-permafrost hydrate-bearing sediments means that certain parameters play a particularly important role. These include

- petrophysical properties for the sediment

$\bigcirc$ the characteristic drainage and imbibition curves (capillary pressure vs. saturation)

$\circ$ relative permeability curves for the gas and brine phases

- geological properties

$\circ$ grain size distribution and its variation within the sediment,

- driving forces

$\circ$ capillary pressure of the gas phase and its variation with position and with gas column height 
- gradients in saturation and capillary pressure.

\section{Key Findings}

Key findings for sub-permafrost hydrate-bearing sediments include:

- Variation in grain size distribution within a sediment layer can give rise to a hydrate saturation profile where sand-rich intervals containing little hydrate are interspersed between intervals containing large hydrate saturations.

- Viewed from the pore scale, conversion of a gas reservoir to hydrate is an imbibition process because the gas phase pressure decreases as the gas column height decreases. A pore-scale simulation of imbibition that assumes water reaches the gas-water interfaces very slowly yields an upper bound on final hydrate saturation of $S_{h, \text { final }}=S_{\text {g, initial }}$. Repeating the imbibition simulation with the assumption that water reaches interfaces very rapidly yields a lower bound on $S_{h, f i n a l}$. These bounds bracket saturation profiles reported for Mt Elbert and for Mallik.

- Viewed from the bed scale, conversion of a gas reservoir to hydrate is a transport problem: Large volumes of gas phase and brine phase (about two thirds of a pore volume of each phase) must enter the hydrate-bearing sediment during hydrate formation. A meter-scale laboratory experiment confirms that significant volumes of fluid phases move into the hydrate stability zone.

- Of the fluids that enter the hydrate-bearing sediment, the fraction which is gas determines the final hydrate saturation.

- The rate of hydrate formation, which is related to the rate of descent of the base of gas hydrate stability zone, and the relative permeabilities of gas and aqueous phases determine the gas phase fraction in the total fluids moving to the hydrate-bearing sediment.

- The accumulation of large hydrate saturations, like those reported at Mt Elbert and Mallik, require gas and brine phase transport from below the hydrate stability zone. This transport can be driven by capillarity along saturation gradients.

- Capillarity-controlled transport in turn requires relatively slow descent of the base of the gas hydrate stability zone. Otherwise, the rate of hydrate formation demands faster transport, which requires viscous forces to play a stronger role. Transport dominated by viscous forces approaches the solution given by fractional flow theory, in which a gas bank forms and aqueous phase cannot reach the hydrate stability zone, stopping the generation of hydrate at saturations much smaller than observed at Mt Elbert and Mallik.

Key findings for deep-water hydrate-bearing sediments include:

- "Capillary fracturing" is a dominant gas transport mechanism in low-permeability media.

- This phenomenon has been proposed based on grain-scale simulations in which we extended a discrete element mechanics code (PFC, by Itasca) to incorporate the dynamics of first single-phase and then multiphase flow, but was then confirmed experimentally in a set of laboratory experiments that demonstrate the different regimes of gas invasion in deformable sediments (capillary fingering, viscous fingering, and fracturing). 
- Gas invasion regime is characterized by two dimensionless groups: a modified capillary number and a "fracturing number" that reflects the balance between the pressure forces that act to open conduits in the granular pack, and frictional forces that resist it.

- The morphology of the gas-invaded region exerts a fundamental control on the fabric of methane hydrate formation, and on the overpressures caused by methane hydrate dissociation.

- Methane transport in lake and oceanic sediments is controlled by dynamic conduits, which dilate and release gas as the falling hydrostatic pressure reduces the effective stress below the tensile strength of the sediments. This conceptual and mathematical model was tested against a four-month record of hydrostatic load and methane flux in Upper Mystic Lake, Mass., USA, and show that it captures the complex episodicity of methane ebullition.

- Our quantitative conceptualization opens the door to integrated modeling of methane transport to constrain global methane release from lakes and other methane-rich sediment systems, and to assess its climate feedbacks. 


\section{Development and Application of Bed Scale Models}

Our analysis of pore-scale phenomena in this project has delineated three regimes that govern processes in which the gas phase pressure is increasing: fracturing, capillary fingering and viscous fingering. These regimes are characterized by different morphology of the region invaded by the gas (see Figure below).

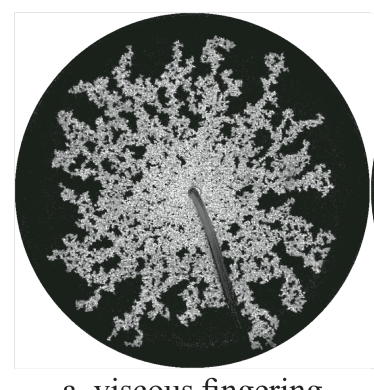

a. viscous fingering

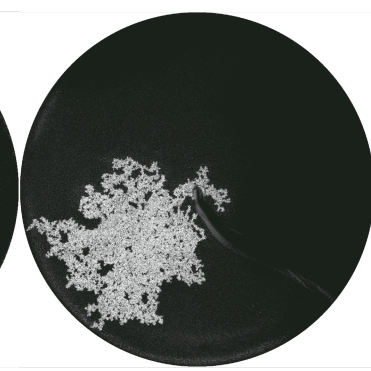

b. capillary fingering

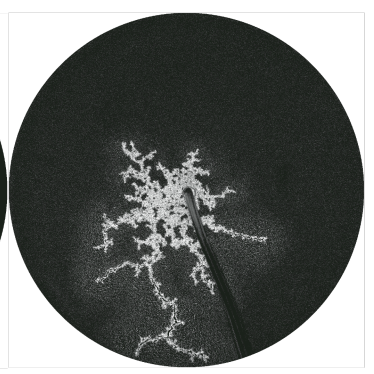

c. fracturing

Figure. Different gas invasion regimes, observed experimentally [Holtzman et al., 2012]

The capillary fingering regime corresponds to a drainage displacement. On the other hand when the gas phase pressure is decreasing, the corresponding regimes are capillary fingering and compaction. In this case, the fingering corresponds to an imbibition displacement. Compaction corresponds to closure of fractures and tighter packing of grains. In this project, we studied all these regimes except compaction.

This section is the body of this report. It comprises two large subsections, one on Capillarity Dominated Behavior, the other on Fracturing Dominated Behavior.

The application focus of the Capillarity Dominated Behavior subsection is on subpermafrost accumulations of hydrate. Large saturations of hydrate in sand-rich sediments in the Arctic have drawn great interest. The leading hypothesis for the creation of these accumulations that they are converted from existing gas reservoirs. The conversion to hydrate occurred when the base of gas hydrate stability descended through the reservoirs. The detailed mechanism by which this conversion occurs has not been elucidated. A major contribution of the research conducted in this project is the first quantitative and mechanistic model of the conversion process. We describe this process and several possible models. We show that field observations at Mt Elbert can be represented well by the limiting case of a closed system in which gas phase pressure decreases.

In the section on Fracturing Dominated Behavior we turn to cases in which gas phase pressure is increasing. The application focus is quite broad: relatively shallow sediments of relatively small permeability which are highly susceptible to fracturing as gas phase pressure increases. We demonstrate the existence of the different invasion regimes by means of controlled laboratory experiments in a radial cell. We collapse the behavior in the form of a phase diagram fully characterized by two dimensionless groups: a modified capillary number and a "fracturing number" that reflects the balance between the pressure forces that act to open conduits in the granular pack, and frictional forces that resist it. We use all this small-scale knowledge to propose simple mechanistic models of gas migration and hydrate formation at the geologic bed scale. We propose that methane transport in lake and oceanic sediments is controlled by dynamic conduits, which dilate and release gas as the falling hydrostatic pressure reduces the effective stress below the tensile strength of the sediments. 


\section{Capillarity Dominated Behavior}

\section{Geologic Context for Capillarity-Dominated Processes in Sub-Permafrost Hydrate-Bearing Sediments}

Prospecting for gas hydrates as a resource has only recently begun to adopt the perspective of exploring for petroleum systems (Collett et al., 2009). Gas hydrates are formed at low temperatures and generally high pressures (e.g. temperatures below $25^{\circ} \mathrm{C}$ and pressures greater than 1.5 MPa for natural gas hydrates) which are typical of relatively shallow depths in oceanic sediments (Kvenvolden, 1988; Koh et al., 2002) or deeper sediments in the Arctic (Collett, 1993).

In deep ocean sediments hydrates occur in various morphologies (Sloan, 1998; Waite et al., 2009). Hydrates have been observed as disseminated grains filling pores (Dallimore and Collett, 2005; Fujii et al., 2008) and as complex networks of filled fractures and veins in fine grained sediments (Hadley et al., 2008; Holland et al., 2008). Beneath the Indian Ocean (offshore India) most of the recovered gas hydrate was found to exist in "combination reservoirs", characterized as either pore-filling grains or particles disseminated (in coarser grain sediment) or as fracturefilling (in clay dominated sediments) (Collett et al., 2009).

In the Arctic such as Alaska's North Slope (ANS), strata several meters thick, containing large saturations $(65 \%-75 \%)$ of gas hydrate are often separated by layers of varying thickness that contain little or no hydrate (Boswell et al., 2011). In addition, gas hydrates in permafrost regions have been commonly reported to occur with pore-filling morphology in sand-rich sediments (Dallimore and Collett, 2005).

Hydrate formation in sediments, especially in marine environments, has been subject to series of analyses. Predictive models of the process can be divided into two categories: (A) Models assuming formation of hydrate from methane dissolved in water in which accumulation is driven by methane-saturated water entering the gas hydrate stability zone (GHSZ) or by providing methane from a biogenic source (Hyndman and Davis, 1992; Ginsburg, 1998; Xu and Ruppel, 1999; Buffett, 2000; Hensen and Wallmann, 2005; Bhatnagar et al., 2007) and (B) models assuming formation of hydrate at the interface between gaseous and aqueous phases in which accumulation is driven by methane gas phase entering the GHSZ (Torres et al., 2004; Liu and Flemings, 2006, 2007). One motivation for the latter class of models was that observed chloride concentrations and gas hydrate distributions could not be explained without assuming transport of free gas through GHSZ (Torres et al., 2004).

The model categories (A) and (B), address marine hydrate reservoirs. While there is no comparably mechanistic model of hydrate formation for terrestrial hydrate accumulations, i.e. subpermafrost hydrate reservoirs, in the literature, the overall process has been delineated. Boswell et al. (2011) listed several aspects of gas hydrate accumulations in the Arctic that support the interpretation of conversion of free gas accumulation to gas hydrate accumulations. The accepted scenario for Arctic hydrate reservoirs such as those of the Prudhoe Bay and Kuparuk River area in ANS is that gas first accumulated in shallow traps when those traps were below the GHSZ. The gas reservoirs moved into the GHSZ in response to ancient climate cooling (Collett, 1993). 
In this report a more detailed model of the process of hydrate accumulation is proposed, based on the latter Arctic scenario. A major difference between this model and the marine models (A) and (B) is that instead of presuming fluxes of fluid(s) to the GHSZ, we presume that the base of GHSZ moves down to (and through) an existing petroleum system. Fluid flux occurs in our model (and plays an important role in determining saturation) but only as a response to BGHSZ motion. This downward movement of the BGHSZ occurred in the ANS roughly $1.8 \mathrm{Ma}$ (Collett, 1993; Dai et al., 2011). For simplicity we also assume that $\mathrm{CH}_{4}$ is the only constituent of the gas phase and the only guest molecule in the hydrate.

One motivation for a detailed process model is the observation in the well-characterized Milne Point Unit (MPU) hydrate accumulations, e.g. in Mount Elbert well, that hydrates are often restricted to the upper part of the sand units (Fig. 1b). Several explanations are possible. In the upper hydrate-bearing sand unit of Mount Elbert well, known as Unit D (Collett, 1993), this observation could be interpreted as the occurrence of high quality (large porosity and large intrinsic permeability) sand at the top of the unit. However, in the lower hydrate-bearing sand unit, known as Unit C (Collett, 1993), this is not the case (Boswell et al., 2011). The top section of Unit $\mathrm{C}$ contains the major hydrate saturation (Fig. 1b) while it possesses a significantly lower porosity and intrinsic permeability than the lower section containing little or no hydrate (Boswell et al., 2011). Moreover, reservoir sands in the MPU are only partially filled with hydrate and often exhibit a sharp basal contact. For example within the D Unit log and core data exhibit an abrupt decrease in hydrate saturation, $S_{h}$, from greater than $65 \%$ to less than $10 \%$ despite the gradational decrease in reservoir quality, reduced porosity and increased shale content (Boswell et al., 2011). Hence it is difficult to postulate a straightforward relation between reservoir quality and hydrate saturation in the Mt. Elbert well. 


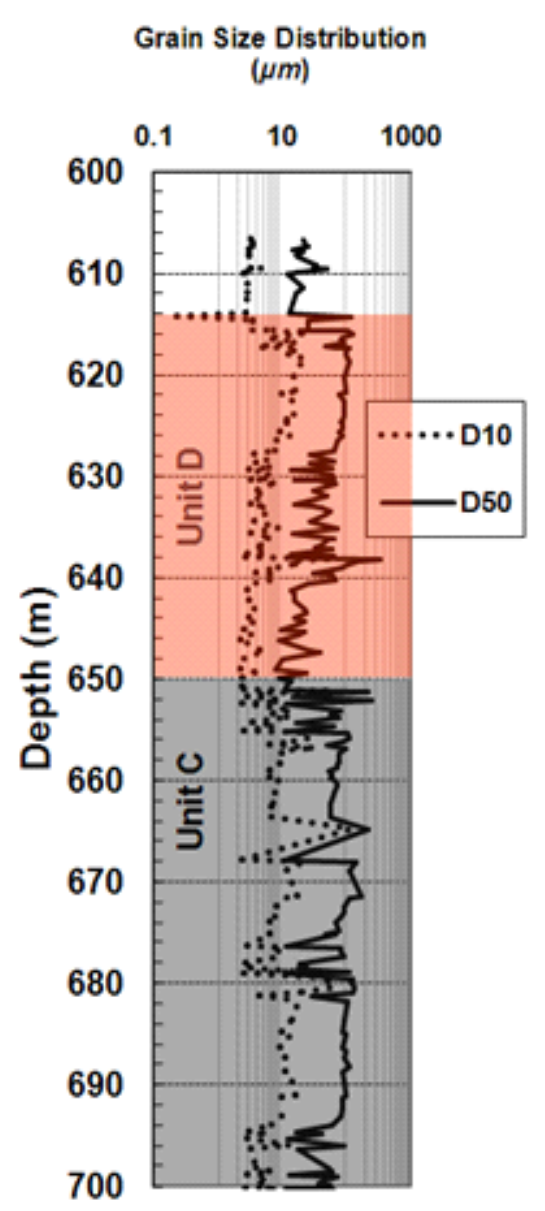

(a)

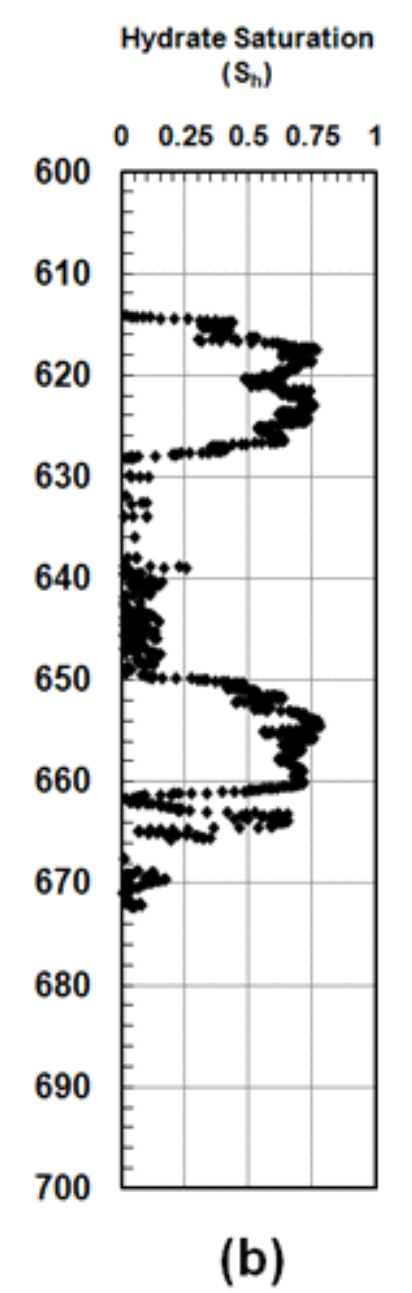

Fig. 1. Data from Mount Elbert stratigraphic test well, drilled in the Milne Point unit of Alaska North Slope: (a) $10^{\text {th }}$ percentile, $D_{10}$, and $50^{\text {th }}$ percentile, $D_{50}$, of grain size distribution versus depth determined from laser-grain-size analyses (Rose et al., 2011); (b) Gas hydrate saturation, $S_{h}$, determined from the TCMR-repeat-pass-plus-density log based on NMR-DEN POR method (Lee and Collett, 2011). The shown interval of interest includes the informally labeled C and D units of Collett (1993).

Another possible explanation of these variations in hydrate saturation is that they simply reflect the variation of the original gas saturation distribution. Unit D may have been only partially filled with gas for a variety of reasons - a shallow spill point, the stratigraphic seal could hold only a modest gas column, or an interruption in the gas migration path caused insufficient charge, etc. But partial charge is not a necessary condition for variable hydrate saturation in an individual reservoir: the volume changes associated with hydrate formation mean that hydrate will occupy only the upper portion even of a fully charged reservoir. Whatever the extent of charge, this explanation assumes that the conversion to hydrate occurs after the reservoir is no longer connected to the source of gas.

The partial charge explanation becomes more critical in the case of a vertical sequence of partially filled hydrate reservoirs. This explanation would require Units $\mathrm{D}$ and $\mathrm{C}$ to have been charged separately, for example by migration along the individual layers from a conductive downdip fault connected to the gas source. It would also require no vertical communication between the gas accumulations, i.e. a sufficiently large capillary entry pressure for the sediment 
at the base of Unit D. The requisite entry pressure depends in turn on the height of gas column resulting from the charge of Unit $\mathrm{C}$.

Because the conversion to hydrate drives substantial fluid migration, the structural setting of the gas accumulations also affects the final hydrate distribution. The model analyzed here is for the limiting case of horizontal layers, in which fluid migration is only in the vertical direction. In this case the process is essentially one-dimensional. This is a reasonable approximation for the Mount Elbert prospect. The C and D Units occur within the non-marine Tertiary Sagavanirktok Formation and are laterally extensive throughout the Milne Point Unit (MPU) and beyond (Boswell et al., 2011) covering approximately $360 \mathrm{~km}^{2}$ (Collett, 1993; Winters et al., 2011). A seismic survey (Inks et al., 2009) suggests that hydrate-bearing intervals in the Mt. Elbert prospect are quite extensive in terms of areal distribution. The Mt. Elbert well is located on a monoclinal structure that dips less than $2^{\circ}$ to the NNE (Collett, 1993; Inks et al., 2009; Boswell et al., 2011). The BGHSZ itself can be approximated as a horizontal surface, so its descent through laterally extensive gas accumulations within a nearly horizontal structure is also an approximately one-dimensional process. In this limiting case hydrate will form uniformly within the GHSZ, and any gas flow induced during hydrate formation must come from the accumulation still below the GHSZ. As the dip angle increases, gas can migrate along dip as well as vertically; the limiting case treated here provides a lower bound on the saturation profile for dipping beds. Conductive faults provide another possible route for fluid migration at Mt. Elbert well on the upthrown side of a large normal fault (Boswell et al., 2011) which may have contributed to the flow at the time of hydrate formation, but we assume all faults are sealing in this work.

Thus the timing, the pathway, the extent of charge and the dip angle are key controls on final hydrate distribution in a multilayer column of sediment. The model presented here makes the partial charge explanation quantitative for noncommunicating layers, and more importantly it generalizes the partial charge model to show how a stack of partially filled hydrate reservoirs can result even from initially communicating layers that host a single continuous column of gas.

To summarize, applying the proposed 1-D model on field data from Mount Elbert well, we show that certain characteristic features associated with hydrate occurrence in the Arctic are consistent with three conditions: i) the establishment of gas phase saturation within the sediment when the base of gas hydrate stability zone (BGHSZ) was located above the sediment package; ii) sufficient variation of grain size distribution with depth and iii) volume reduction during hydrate formation, i.e. hydrate occupies less volume than its constituents $\left(\mathrm{CH}_{4}, \mathrm{H}_{2} \mathrm{O}\right)$ in their respective phases. The latter is presumably true in all natural hydrate-bearing sediments, including Arctic sediments. The first condition is also commonly satisfied in the Arctic; the second depends on local depositional history.

\section{Physical Phenomena Critical to the Proposed Model}

Grain size varies with depth in most depositional environments. When gas accumulates in a sediment, these variations play an important role on the gas/water saturation profile. Fig. 2a shows schematically a stack of four distinct sediment layers with different grain size distributions. Each layer thus has a different characteristic curve of capillary pressure versus saturation, as shown in Fig. 2b. Capillary pressure, $P_{c}$, is defined as the difference between the non-wetting phase pressure, in this case gas pressure $P_{g}$, and wetting phase, aqueous phase pressure $P_{w}$. Capillary pressure curve shows the relationship describing the capillary pressure required for establishing a given gas phase saturation in a sediment originally filled with water. 
The top layer (layer 1) has the smallest grains, and the corresponding capillary pressure curve shows a much larger capillary entry pressure than other layers. If gas enters this stack of sediments from the bottom of layer 4 (Fig. 2a) and begins to accumulate, the capillary pressure increases with gas column height above the entry point as shown in Fig. 2c. The capillary pressure at any height combined with the corresponding drainage curve (Fig. 2b) yields the gas/water saturation profile shown in Fig. 2d. The fine-grained layer 1 acts as a seal for the gas accumulation in Fig. 2d. Note that the BGHSZ is above the gas column and thus no hydrate is present in Fig. 2d. Fig. 2e shows the situation when the BGHSZ has descended all the way down the gas column and thus the gas accumulation (Fig. 2d) has turned into hydrate. Note that the initially single gas column (Fig. 2d) turned into discrete, concentrated hydrate deposits at the top section of two of the formations (Fig. 2e). The resulting hydrate saturation profile in Fig. 2e exhibits a similar distribution to the Mount Elbert well (Fig. 1b). More detailed explanation of how vertical heterogeneity, i.e. grain size variation versus depth, can lead to such discrete, concentrated hydrate deposits is provided in a later section.

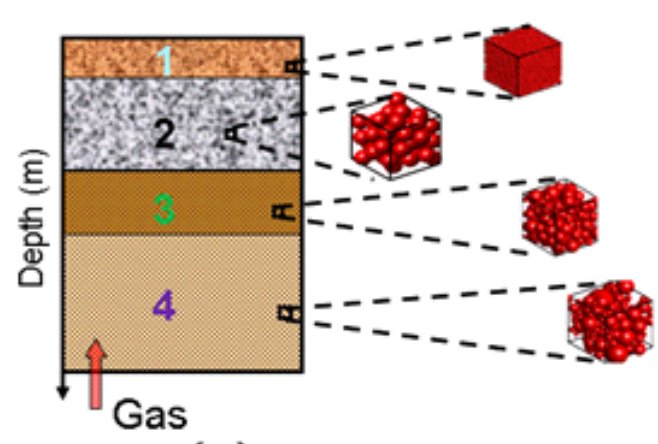

(a)

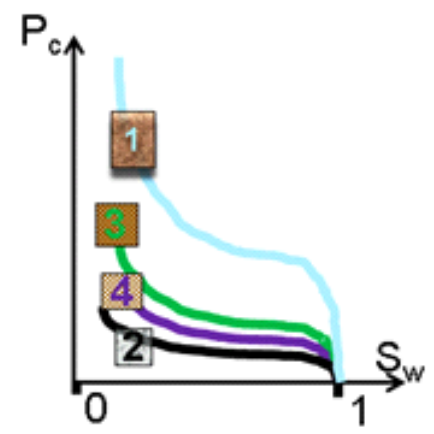

(b)

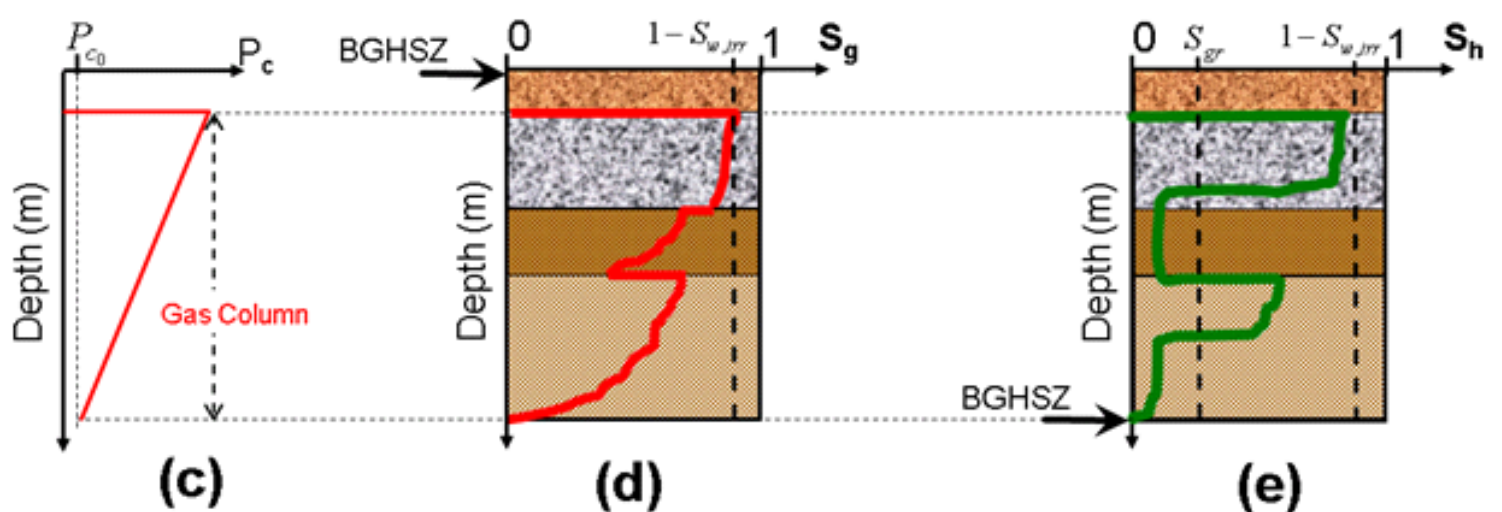

Fig. 2. (a) Sediment layers with different grain size distributions. The characteristic capillary pressure for each layer is shown in (b). Gas enters the bottom layer and accumulates below the fine-grained layer at top of sediment package. (c) Capillary pressure profile within the gas column, combined with the characteristic curves of (b), determines the gas saturation profile (d) through the sediments. Note that gas accumulation has occurred while BGHSZ is shallower than the gas column and thus no hydrate is being formed so far. (e) As BGHSZ descends along the sediment column, a hydrate saturation profile is established which can be very different from the initial gas saturation profile and need not correlate with sediment layering.

The other physical phenomenon essential to our model is that the volume occupied by a mole of methane hydrate, i.e. the amount of hydrate containing one mole of gas, is smaller than the 
volume occupied by gas and aqueous phases containing the same number of moles of $\mathrm{CH}_{4}$ and $\mathrm{H}_{2} \mathrm{O}$ as the hydrate. This reduction in volume accompanying hydrate formation follows from hydration number, $N$, in the hydrate lattice, $\mathrm{CH}_{4} \cdot N\left(\mathrm{H}_{2} \mathrm{O}\right)$, and typical densities of the respective phases. Hydrates are known to be non-stoichiometric, meaning that $N$ varies with pressure, temperature and overall gas composition (Sloan, 2003), with values of $N=6.05 \pm 0.6$ (Handa, 1986; Ripmeester and Ratcliffe, 1988) for methane hydrate. For simplicity we use a constant value of $N=6$, which is consistent with an average density of $914 \mathrm{~kg} / \mathrm{m}^{3}$ for methane hydrate (Sloan, 1998). As a numerical example of the volume reduction associated with hydrate formation, consider a vessel containing $0.5 \mathrm{~m}^{3}$ of methane and $0.5 \mathrm{~m}^{3}$ of pure water held at a constant pressure of $6.5 \mathrm{MPa}$ and temperature of $2{ }^{\circ} \mathrm{C}$ during hydrate formation. At these conditions the phase densities are about $55 \mathrm{~kg} / \mathrm{m}^{3}$ (gas) and $1000 \mathrm{~kg} / \mathrm{m}^{3}$ (aqueous). For $N=6$, the molecular weight of hydrate would be 124 . All the gas will be consumed to form hydrate, and the hydrate volume is $V_{h}=0.5 \times \frac{55}{16} \times \frac{124}{914} \approx 0.23 \mathrm{~m}^{3}$. It can be shown that almost $0.18 \mathrm{~m}^{3}$ of water is used to form the latter amount of hydrate. Therefore, at the current thermodynamic condition the final hydrate volume, $0.23 \mathrm{~m}^{3}$, is almost one third of the consumed volumes of methane and water combined.

Conceptually, as hydrate forms from a fixed volume of gas phase in a sediment, void space becomes available. Here "void space" is shorthand for the volume change accompanying hydrate formation. The void space will either be filled by influx of fluid phase(s) or accommodated by compaction of the sediment. Here, we assume no compaction occurs during conversion to hydrate. We will see that in any case the volume changes are too large to be accommodated only by compaction. Methane which is transported to the GHSZ to help fill the void space is then converted to hydrate inside the GHSZ. As discussed below, the relative ratio of transported gaseous and aqueous phases affects the resulting hydrate saturation profile.

The rate of salinity transport can also affect the hydrate saturation profile. The inclusion of $\mathrm{H}_{2} \mathrm{O}$ into the hydrate causes salinity to build up in the aqueous phase, potentially reaching the critical value at which hydrate is no longer stable at prevailing temperature and pressure. Here we assume that the aqueous phase has a small initial salinity. Furthermore, the descent of BGHSZ is assumed to occur over geologic time scales and thus any salinity buildup is dissipated into an unlimited reservoir of brine. Considering that heat diffusion (typically $10 \mathrm{~m}^{2} / \mathrm{yr}$ ) is about 500 times faster than salinity transfer (typically $0.02 \mathrm{~m}^{2} / \mathrm{yr}$ ) (Iversen and Jorgensen, 1993; Clennell et al., 1999), we also neglect the effect of heat transfer.

We focus on the conversion of an accumulated gas saturation to hydrate as the BGHSZ moves down. Subsequently, hydrate dissociates if the BGHSZ moves upwards, leaving the gas bearing region out of GHSZ. Cycles of hydrate formation and dissociation could take place during geological time. For example, such movements of BGHSZ are reported in MacKenzie delta region (Majorowicz et al., 2008; Torres et al., 2011). Similar oscillations of the BGHSZ in Mount Elbert well were reported by Dai et al. (2011); however, the hydrate-bearing section of the Mount Elbert well remained well inside the GHSZ and not disturbed by the oscillations of the BGHSZ.

\section{Volume Change for Hydrate Formation from Gas and Water}

A box model is used to compute the volume change as hydrate forms. The box (with a volume $V_{t o t}$ ) is originally filled by methane and water existing as two pure phases (Fig 3a). The initial gas/water volumes are prescribed and no hydrate is present initially. Temperature and 
pressure are then adjusted to values at which hydrate is stable and held constant during hydrate formation. Phase densities are thus constant.

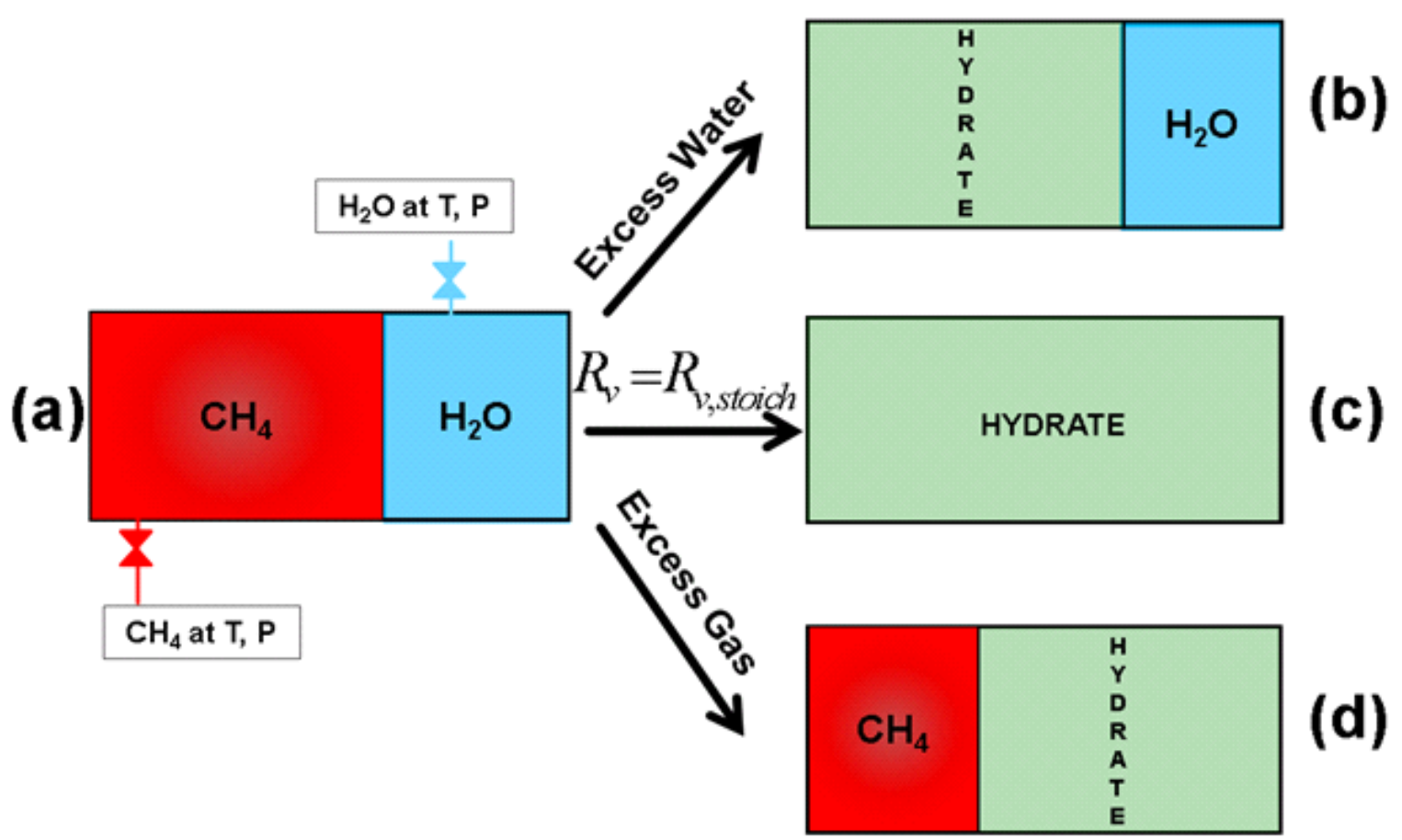

Fig. 3. (a) Hydrate formation in an open system: prescribed volumes of $\mathrm{CH}_{4}$ and $\mathrm{H}_{2} \mathrm{O}$ phases are initially present, and $\mathrm{CH}_{4}$ and $\mathrm{H}_{2} \mathrm{O}$ can enter so that $\mathrm{T}, \mathrm{P}$ are constant during hydrate formation; (b) final state when the total amount of water (initial amount + the amount entered) is more than the stoichiometric requirement (excess water); (c) final state when the total amount of gas and water are at the exact stoichiometric ratio; (d) final state of the open system when the total amount of gas (initial amount + amount entered) is more than the stoichiometric gas requirement (excess gas).

Suppose an incremental volume of hydrate $\delta V_{h}$ forms at the interface between gas and water phases as the result of stoichiometric, assuming constant hydration number $N=6$, conversion of the corresponding increments of methane and water, $\delta V_{g}$ and $\delta V_{w}$. For typical hydrate-stability subsurface conditions the phase densities are such that $\delta V_{h}<\delta V_{g}+\delta V_{w}$, and we write:

$\delta V_{h}+\delta V=\delta V_{g}+\delta V_{w}$

Here $\delta V$ denotes the net change in volume occupied by fluids and hydrate. The consequence of this volume change depends on whether the box is closed or open.

For a closed box the only way to keep the pressure and temperature constant is by changing the volume of the box, i.e. $V_{\text {tot }}$ decreases by $\delta V$. This would correspond to compaction (grain rearrangement to reduce porosity) in a natural system.

The sediments of interest behave more like open systems (fluid phases can leave or enter). In the model of Fig. 3, both water and methane can enter the box as needed to compensate for the volume reduction during hydrate formation and thus maintain the pressure. Hydrate will keep forming until one of the components (methane, water) inside the box is fully consumed. We define $R_{n}$ as the ratio of number of gas phase moles, $\Delta n_{g}$, to the total number of moles of gas and aqueous phase, $\Delta n_{g}+\Delta n_{w}$, transported into the box. Two cases can be considered in terms of the initial and transported amounts of water, $n_{w, i}$ and $\Delta n_{w}$, and of methane, $n_{g, i}$ and $\Delta n_{g}$ : 


\section{Excess Water: Total Water Exceeds Stoichiometric Requirement}

In this case the total number of moles of water, $n_{w, i}+\Delta n_{w}$, is more than the stoichiometric amount of water required to convert the total amount of methane into hydrate, $N\left(n_{g, i}+\Delta n_{g}\right)$. Therefore, all the gas is consumed to form $n_{h}$ moles, equivalent to a volume $V_{h}$, of methane hydrate and thus,

$$
V_{w, f}=V_{t o t}-V_{h}
$$

where, $V_{\text {tot }}$ denotes the total volume of the box and $V_{w, f}$ is the final water volume inside the box. Furthermore,

$$
n_{h}=\frac{V_{h}}{\bar{V}_{h}}=\left(n_{g, i}+\Delta n_{g}\right)=\frac{V_{g, i}+\Delta V_{g}}{\bar{V}_{g}}
$$

where $V_{g, i}$ denotes the initial gaseous phase volume in the box and $\Delta V_{g}$ is the gaseous phase volume transported into the box. $\bar{V}_{h}$ and $\overline{V_{g}}$ are the molar volumes of hydrate and methane, respectively. Molar volume of a component is equal to the molecular weight of the component divided by its density, i.e.:

$\bar{V}_{c}=\frac{M W_{c}}{\rho_{c}} ; c=w, g, h$

The final volume occupied by aqueous phase would be:

$$
V_{w, f}=V_{w, i}+\Delta V_{w}-\underbrace{N \times V_{h} \frac{\bar{V}_{w}}{\bar{V}_{h}}}_{{\text {Consumed } H_{2} O}^{O}}
$$

where $V_{w, i}$ and $V_{w, f}$ denote the initial and final water volume inside the box, respectively. $\Delta V_{w}$ is the volume of the aqueous phase transported into the box and $N$ is the hydration number. Rewriting the right hand side of Eq. (5) in terms of moles yields:

$$
V_{w, f}=\left[n_{w, i}+\Delta n_{w}-N\left(n_{g, i}+\Delta n_{g}\right)\right] \bar{V}_{w}
$$

The hydrate volume can be written in terms of the initial moles of gas in the box and the molar ratio of gas transported, $R_{n}=\Delta n_{g} /\left(\Delta n_{g}+\Delta n_{w}\right)$, into the box:

$V_{h}=\left[\frac{R_{n}\left(\bar{V}_{g}-\bar{V}_{w}\right)+\bar{V}_{w}}{R_{n}\left(\bar{V}_{h}-(N+1) \bar{V}_{w}\right)+\bar{V}_{w}}\right] n_{g, i} \bar{V}_{h}$

Figure $3 b$ illustrates a typical final state of the system in the case of excess water. Equations (2) and (5) give the total volume of water that entered the box, in terms of the final hydrate volume:

$$
\Delta V_{w}=V_{t o t}-V_{w, i}+V_{h}\left(N \frac{\bar{V}_{w}}{\bar{V}_{h}}-1\right)
$$


Similarly, Eq. (3) gives the total volume of gas that entered the box:

$\Delta V_{g}=\frac{\bar{V}_{g}}{\bar{V}_{h}} V_{h}-V_{g, i}$

Equations (8) and (9) give the total phase volume transported into the box, $\Delta V_{w}+\Delta V_{g}$, as a function of the final hydrate volume, $V_{h}$ :

$\Delta V_{w}+\Delta V_{g}=K_{\text {trans }} V_{h}$

where $K_{\text {trans }}=\frac{N \bar{V}_{w}+\bar{V}_{g}}{\bar{V}_{h}}-1$. In fact, $K_{\text {trans }}$ is the total phase (gaseous + aqueous) volume transported into the box (or any hydrate formation zone) per unit volume of hydrate formed, so that the total system volume is fixed. Note that $K_{\text {trans }}$ is independent of the initial amount of gaseous and aqueous phase.

\section{Excess Methane: Total Methane Exceeds Stoichiometric Requirement}

In this case, there is more methane than the amount required to consume the total amount of water to form hydrate, i.e. $n_{g, i}+\Delta n_{g}>\left(n_{w, i}+\Delta n_{w}\right) / N$. Therefore, all the water is consumed to form $n_{h}$ moles, equivalent to a volume $V_{h}$, of methane hydrate. Figure $3 d$ illustrates a typical final state of the box in the case of excess methane. A development analogous to that in Section 2.1.1 yields the final hydrate volume as a function of $R_{n}$ and the volumes of methane and water that enter the box:

$$
\begin{aligned}
& V_{h}=\left[\frac{R_{n}\left(\bar{V}_{g}-\bar{V}_{w}\right)+\bar{V}_{w}}{R_{n}\left((N+1) \bar{V}_{g}-\bar{V}_{h}\right)+\left(\bar{V}_{h}-\bar{V}_{g}\right)}\right] n_{w, i} \bar{V}_{h} \\
& \Delta V_{g}=V_{t o t}-V_{g, i}+V_{h}\left(\frac{\bar{V}_{g}}{\bar{V}_{h}}-1\right) \\
& \Delta V_{w}=N \frac{\bar{V}_{w}}{\bar{V}_{h}} V_{h}-V_{w, i}
\end{aligned}
$$

Equations (12) and (13) show that the total phase volume transported into the box, $\Delta V_{w}+\Delta V_{g}$, is governed by Eq. (10) regardless of having an excess water or excess methane case.

Figure 3c shows a special case where the final state of the system is having hydrate only. This happens only if

$$
\frac{n_{w, i}+\Delta n_{w}}{n_{g, i}+\Delta n_{g}}=N
$$


Combining Eqs. (3) and (14) gives $\Delta n_{g}=\Delta n_{g, s t o i c h}$, required for the special case to happen, as:

$\Delta n_{g, \text { stoich }}=\frac{V_{\text {tot }}}{\bar{V}_{h}}-n_{g, i}$

Equations (14) and (15) give:

$\Delta n_{w, \text { stoich }}=N \frac{V_{\text {tot }}}{\bar{V}_{h}}-n_{w, i}$

Equations (15) and (16) show that the value of $R_{n}$ for this special case, i.e. $R_{n, \text { stoich }}=\Delta n_{g, \text { stoich }} /\left(\Delta n_{g, \text { stoich }}+\Delta n_{w, \text { stoich }}\right)$, depends on the initial state, $n_{g, i}$ and $n_{w, i}$. For a given initial phase content, $R_{n}<R_{n \text {,stoich }}$ represents an excess aqueous phase case while $R_{n}>R_{n, \text { stoich }}$ shows an excess gas case.

Based on the formulations above and assuming $V_{t o t}$ is fixed, two independent parameters exist. For example, one choice of independent parameters is $n_{g, i}$ and $\Delta n_{g}$, from which a final $V_{h}$ and $V_{g, f}$ (or $V_{h}$ and $V_{w, f}$ depending on values of independent parameters) is obtained. Another choice is $n_{g, i}$ and $V_{h}$ from which $\Delta n_{g}$ and $\Delta n_{w}$ and thus $R_{n}$ is obtained.

We now write the box model in terms of phase saturations in a volume of sediment. To do so, one pore volume of the sediment (porosity times total sediment volume) can be considered as the open box in the model. Dividing the preceding expressions by $V_{t o t}\left(=1\right.$ pore volume), $V_{g, i}, V_{g, f}$, $V_{w, i}, V_{w, f}$ and $V_{h}$ are replaced by phase saturations, $S_{g, i}, S_{g, f}, S_{w, i}, S_{w, f}$ and $S_{h}$, respectively. Similarly, the transported phase volumes $\Delta V_{w}$ and $\Delta V_{g}$ are replaced by dimensionless volumes, $\Delta V_{w, d}$ and $\Delta V_{g, d}$. For instance, Eq. (10) can be extended to calculate the total pore volumes of gas and aqueous phase transported into a sediment as a function of hydrate saturation, as in Eq. (17).

$\Delta V_{g, d}+\Delta V_{w, d}=K_{\text {trans }} S_{h}$

We define $R_{v}$ as a volumetric version of $R_{n}$, convenient for calculations in sediments, as $R_{v}=\Delta V_{g} /\left(\Delta V_{g}+\Delta V_{w}\right)=\Delta n_{g} \bar{V}_{g} /\left(\Delta n_{g} \bar{V}_{g}+\Delta n_{w} \bar{V}_{w}\right)$. It can be shown that $R_{v}$ and $R_{n}$ are related as: $R_{v}=\frac{R_{n} \bar{V}_{g}}{R_{n}\left(\bar{V}_{g}-\bar{V}_{w}\right)+\bar{V}_{w}}$

When the model is applied to gas accumulations in sediment, two independent parameters are constrained accordingly. For example, one constrained parameter is the initial gas saturation inside the sediment, $S_{g, i}$. However, constraining $\Delta V_{g}$ (or $R_{v}$ ) requires more study. Here we treat $R_{v}$ as a free parameter and examine how system behavior changes with $R_{v}$. An alternative is to consider $S_{g, i}$ and $S_{h}$ as constrained parameters and determine $R_{v}$ (or $\Delta V_{g}$ and $\Delta V_{w}$ ).

Combining Eqs. (7) and (18) and replacing volumes with saturations, gives the final hydrate saturation for the excess water case as a function of $R_{v}$ and initial gas saturation, $S_{g, i}$, as: 
$S_{h}=\frac{\bar{V}_{h}}{R_{v}\left(\bar{V}_{h}-\bar{V}_{g}-N \bar{V}_{w}\right)+\bar{V}_{g}} S_{g, i}$

Similarly, for the excess methane case Eq. (20) holds.

$$
S_{h}=\frac{\bar{V}_{h}}{R_{v}\left(-\bar{V}_{h}+\bar{V}_{g}+N \bar{V}_{w}\right)+\bar{V}_{h}-\bar{V}_{g}}\left(1-S_{g, i}\right)
$$

Figure 4 shows the corresponding curves for a set of values for initial gas/water saturation within a control volume of sediment. Temperature and pressure were assumed $T=2{ }^{\circ} \mathrm{C}$ and $P=6.5 \mathrm{MPa}$ (averaged temperature and averaged hydrostatic pressure over depth along the hydrate-bearing interval in the Mount Elbert well). In fact, the change in gas density due to change in pressure, with depth along the hydrate-bearing interval, is less than 5\%; therefore, for simplicity the density of gas phase was assumed constant and corresponding to the averaged temperature and pressure. Using Peng-Robinson equation of state, methane density was calculated to be $55 \mathrm{~kg} / \mathrm{m}^{3}$. Density of aqueous and hydrate phases were taken as $1000 \mathrm{~kg} / \mathrm{m}^{3}$ and $914 \mathrm{~kg} / \mathrm{m}^{3}$ (Sloan, 1998), respectively. Each curve in Fig. 4 passes through $S_{h}=1$ when $R_{v}=R_{v, \text { stoich }}$. Regardless of the initial phase saturations, for values of $R_{v}<R_{v \text {,stoich }}$ the final state has only hydrate and water phases present (excess water) and for $R_{v}>R_{v, \text { stoich }}$ the final state has hydrate and gas phases only (excess gas).

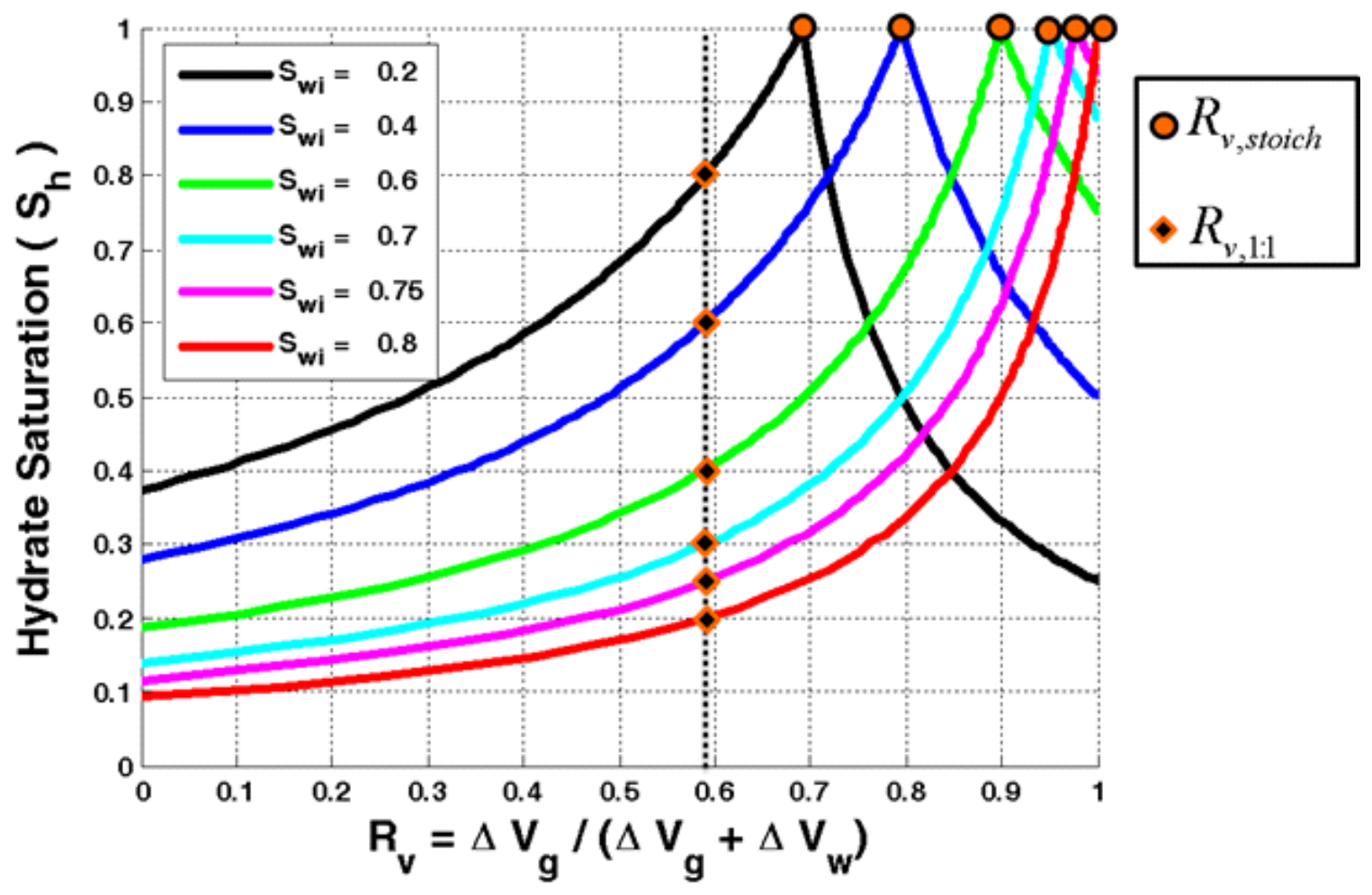

Fig. 4. Final hydrate saturation in an open volume of sediment at $2{ }^{\circ} \mathrm{C}, 6.5 \mathrm{MPa}$ depends strongly on initial water saturation and $R_{v}$, Special points at which $S_{h}$ is the maximum possible value are shown as $R_{v, \text { stoich }}$ on the curves. To the left of $R_{v, \text { stoich }}$ the final state consists of hydrate and water; to the right, of hydrate and gas. Neutral points at which $S_{h}=S_{g, i}$ are shown as $R_{v, 1: 1}$ on the curves, which is equal to 0.59 at this temperature and pressure. 
The value of $R_{v}$ at which $S_{h}=S_{g, i}$ is denoted $R_{v, 1: 1}$, shown as diamonds in Fig. 4 . If the final hydrate saturation $S_{h}$ is larger than initial gas saturation $S_{g, i}$, then the final water saturation $S_{w, f}$ must be less than $S_{w, i}$. In this case the hydrate formation process must have involved drainage (reducing the wetting phase saturation). Conversely, if $S_{h}<S_{g, i}$, thus $S_{w, f}>S_{w, i}$, then the conversion required imbibition. The value of $R_{v, 1: 1}$ thus represents a neutral point between imbibition occurring during hydrate formation for $R_{v}<R_{v, 1: 1}$ and drainage occurring for $R_{v}>R_{v, 1: 1}$. It can be shown that the value of $R_{v, 1: 1}$ depends only on the phase densities and hydration number, $N$ and is independent of initial saturations:

$$
R_{v, 1: 1}=\frac{\bar{V}_{g}-\bar{V}_{h}}{N \bar{V}_{w}+\bar{V}_{g}-\bar{V}_{h}}
$$

\section{Gas reservoir Conversion to Hydrate: Bed-Scale Volumetric Considerations}

Effect of Volume Change During Hydrate Formation on the Resulting Hydrate Saturation Profile from a Finite Column of Gas in a Homogeneous Sediment Column

The above analysis applies to a box of sediment with known initial gas/water saturation. By treating a sediment interval as a column of many such boxes, a given gas/water saturation distribution along the column and a chosen value for $R_{v}$ uniquely determine the hydrate saturation profile. Accumulations are considered as a vertical stack of sediment boxes here since the model is 1-D. However, the latter consideration would not be appropriate for 3-D modeling because the box model does not account for lateral variations in sediment properties.

Consider a column of methane gas and water, in which the gaseous phase is no longer connected to original source of charge, established below a seal in a homogeneous sediment. Figure 5a shows gas saturation versus depth when BGHSZ is at the top of the gas column. As the BGHSZ descends, Fig. 5b, the gas and water above BGHSZ achieve the thermodynamic condition suitable for hydrate formation, hydrate forms, and the model of the preceding section applies.

Figure 5b shows the case in which both gaseous (red arrow) and aqueous (blue arrow) phases enter the vacant space created during hydrate formation. The phase volumes that enter depend on $S_{g, i}$ and $R_{v}$. The methane that enters is then converted to hydrate. As mentioned above, the gas column is assumed disconnected from the original source of charge; therefore, the transported methane is provided from below the hydrate formation zone within the same column of gas. Consequently, a volume of water $\Delta V_{w}^{G W C}$ imbibes from below the gas column to replace the transported gas. This causes the gas-water contact (GWC) to rise, establishing a residual gas saturation at the base of the accumulation, as shown in Fig. 5c. As the BGHSZ descends and the GWC rises, these two levels will meet at a certain depth and thereafter the BGHSZ enters the residual gas zone below the GWC. When the BGHSZ enters the residual gas zone, $R_{v}$ is zero during hydrate formation since no gas can flow at residual gas saturation. The continuation of this process as the BGHSZ descends ultimately yields a hydrate profile with large saturations in the upper portion of the column and small saturations below (Fig. 5d). This profile differs qualitatively from the initial gas saturation profile (Fig. 5a) and is a characteristic result of the model. 


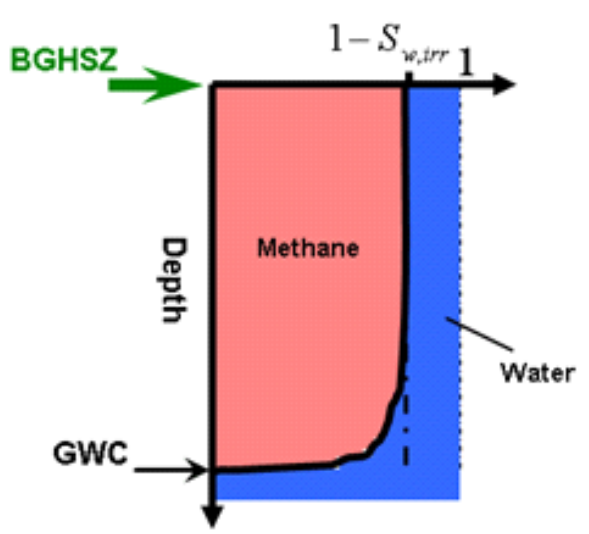

(a)

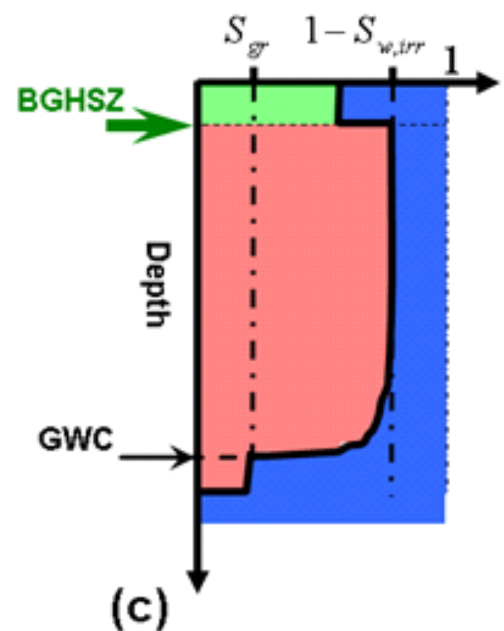

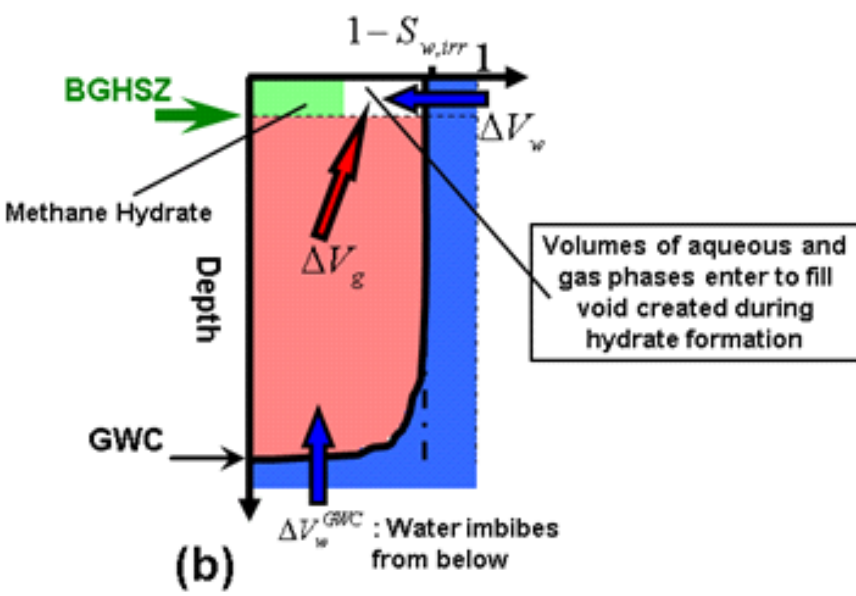

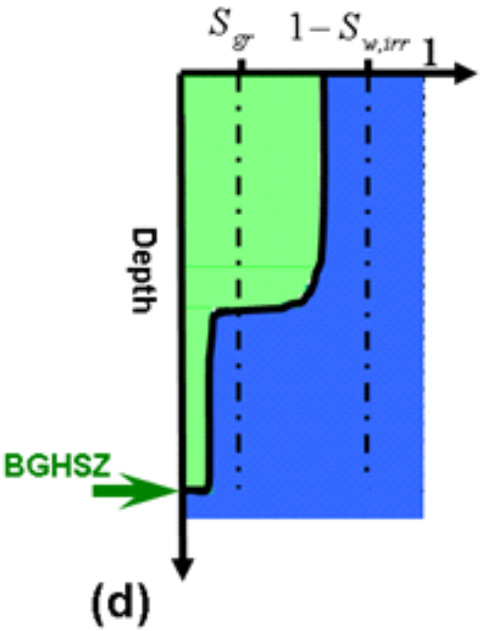

Fig. 5. (a) The initial state of a gas accumulation below a seal in a homogeneous sediment which is below the BGHSZ. (b) After little descent of the BGHSZ. The volume reduction during hydrate formation is shown in white. Gaseous and aqueous phases move into GHSZ to compensate for the volume change. (c) The migrated gas forms more hydrate; the final hydrate saturation in the sediment layer newly located in GHSZ can be estimated from the box model of Figs. 2 and 3 if a value of $R_{v}$ is assumed. (d) The hydrate saturation profile $S_{h}(z)$ after the BGHSZ has descended to the lowermost part of the gas column differs significantly from $S_{g, i}(z)$ of (a). This is a consequence of the volume change during hydrate formation in a finite vertical column of gas.

\section{Effect of Heterogeneity (Grain Size Variation along Depth) on Saturation Distribution}

In contrast to the homogeneous sediment column shown in Fig. 5, heterogeneity is expected in natural sediments. Grain size variation with depth causes variation in capillary entry pressure along the sediment column.

The reduction in gas-water capillary pressure along the gas column, due to the rise of GWC, will have two implications in heterogeneous sediments. First, the gas phase saturation at each depth decreases, with the amount of decrease depending on the characteristic capillary curve for the sediment at each depth. Second, the capillary pressure at one or more depths may fall below the capillary entry pressure for sediment at that depth. If this occurs the initially single column of gas breaks into two (or more) columns of gas no longer in communication with each other. 
Therefore, the capillary entry pressure profile is crucial in our model. Figure 6 illustrates hydrate formation from a pre-established gas accumulation below a seal in a heterogeneous sediment composed of four distinct homogeneous layers (Fig. 6a). The gas accumulation is cooled from the top, i.e. the BGHSZ descends, and consequently converted into hydrate. As the BGHSZ descends, the gas above the BGHSZ as well as some gas migrated from below would form hydrate above the BGHSZ. Similar to that for a homogeneous case, the migrated gas from below is replaced by aqueous phase, resulting in ascent of GWC. The gas-water capillary pressure decreases and intersects the capillary entry pressure at the top of the bottom-most layer and thus the initially single gas column breaks into two non-communicating gas columns: gas columns I and II (Fig. 6b). Therefore, gas column II would not contribute in further hydrate formation within gas column I. When the BGHSZ reaches the top of gas zone II, all the gas above this point is converted into hydrate (Fig. 6c). The final hydrate saturation profile (Fig. 6d), after the BGHSZ has descended to the lowermost part of the gas column, differs significantly from the initial gas saturation profile shown in Fig. 6a. This is a consequence of the volume change during hydrate formation in a finite column of gas, combined with the vertical variation in capillary entry pressure of the host sediment. 


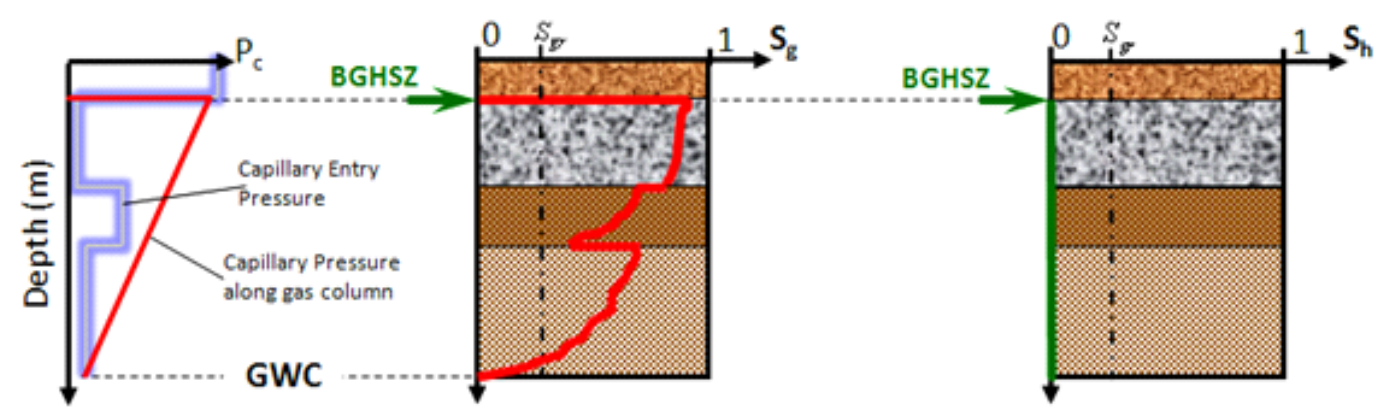

(a)
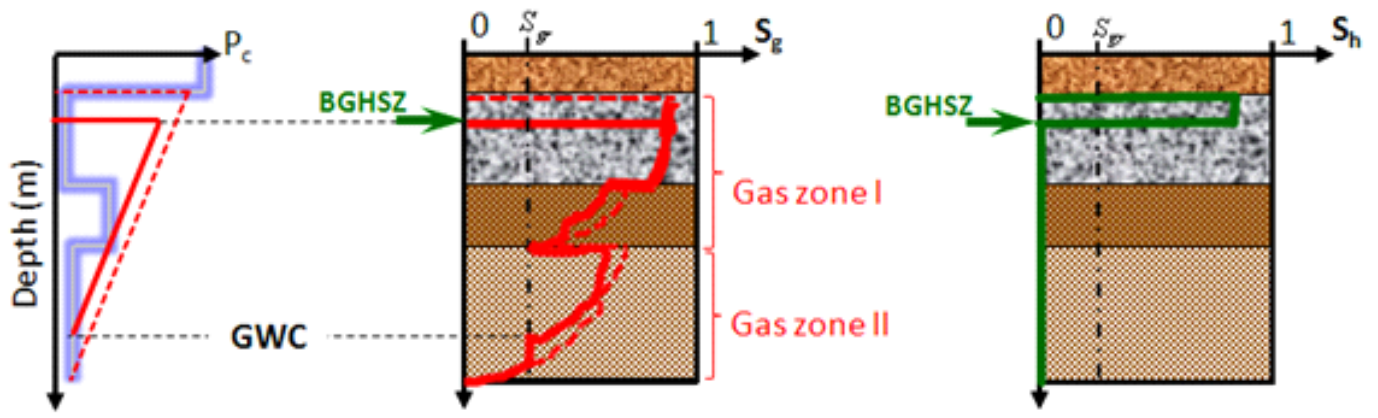

(b)
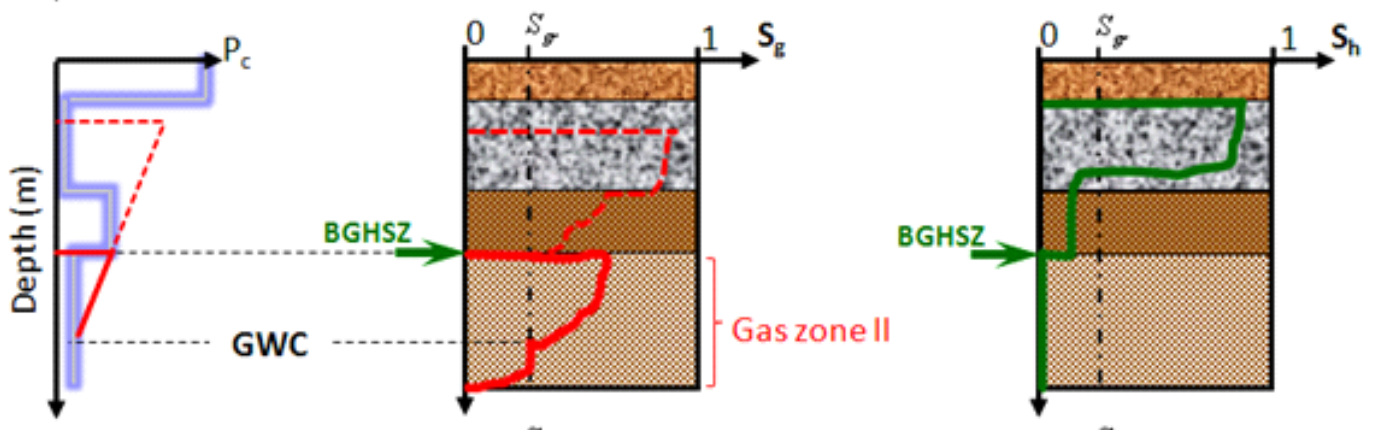

(c)
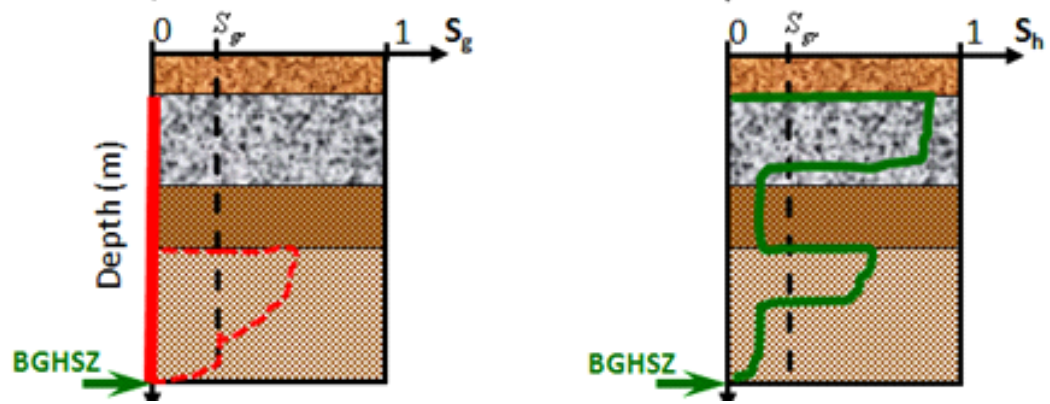

Fig. 6. Hydrate formation from a pre-established gas accumulation in a heterogeneous sediment. The gas accumulation is cooled from the top, i.e. the BGHSZ descends, and consequently converted to hydrate from top to bottom. The left column shows profile of capillary entry pressure versus depth (glowing blue line) as well as profile of capillary pressure along the gas column versus depth (red line). The middle column shows gas saturation versus depth along the sediment, and the right column shows hydrate saturation versus depth. (a) Capillary pressure, gas saturation and hydrate saturation versus depth when the BGHSZ is at the top of the gas column. (b) After little descent of the BGHSZ the gas above the BGHSZ as well as some gas migrated from below would form hydrate above the BGHSZ; the final hydrate saturation in the sediment layer newly located in GHSZ can be estimated from the box model of Figs. 2 and 3 if a value of $R_{v}$ is assumed. The migrated gas from below is replaced by aqueous phase, resulting in ascent of GWC. The capillary pressure decreases and intersects the capillary entry pressure at the top of the bottom-most layer. Therefore, the gas below this point would not contribute in further hydrate formation above this point. Capillary pressure and gas saturation of the previous step is shown in dashed line. (c) When the BGHSZ reaches the top of gas zone II, all the gas above this point is converted into hydrate. (d) The hydrate 
saturation profile $S_{h}(z)$ after the BGHSZ has descended to the lowermost part of the gas column differs significantly from $S_{g, i}(z)$ of (a).

Measurements of capillary entry pressure are rare, but grain size distributions are often available. Thus we develop a simple relationship between grain size parameters and capillary entry pressure.

The capillary entry pressure for a very well sorted granular media has been related to average grain radius, $r_{\text {avg }}$, as:

$$
P_{c, \text { entry }} \approx \frac{6 \sigma}{r_{\text {avg }}}
$$

where $\sigma$ is the interfacial tension between the two fluid phases (i.e. gaseous and aqueous phase). Eq. (22) has been shown both experimentally (Dawe et al., 1992; Mason and Mellor, 1995; Hilpert et al., 2000) and analytically (Mason and Mellor, 1995; Behseresht et al., 2009).

In poorly sorted sediments Eq. (22) is not accurate. However capillary entry pressure is correlated with permeability, which in turn is correlated with grain size distribution. Breyer's equation, Eq. (23), relates hydraulic conductivity, $K$, to grain size distribution in sandy sediments.

$$
K=6 \times 10^{-4} \frac{\rho g}{\mu} \log \left(\frac{500}{U}\right) D_{10}^{2}
$$

where $g$ is acceleration due to gravity, $\mu$ is the dynamic viscosity of the phase flowing through the sediment, $\rho$ is the mass density of the flowing phase, $D_{10}$ is the $10^{\text {th }}$ percentile of grain size in the sediment and $U$, the coefficient of grain uniformity, is defined as:

$U=\frac{D_{60}}{D_{10}}$

Permeability, $k$, is related to hydraulic conductivity by:

$k=K \frac{\mu}{\rho g}$

Combining Eqs. (23) and (25) gives:

$$
k=6 \times 10^{-4} \log \left(\frac{500}{U}\right) D_{10}^{2}
$$

Since Eq. (22) works best for very well sorted granular media, we find an "equivalent" grain size, $r_{e q}$, of a very well sorted sand that has the same permeability as a poorly sorted sediment. The permeability of very well sorted sediments with grain radius, $r$, is (Bryant et al., 1993):

$k=2.7 \times 10^{-3} r^{2}$

Combining Eq. (26) and (27) determines the "equivalent" grain size, $r_{e q}$ :

$r_{e q}=0.4714 \sqrt{\log \left(\frac{500}{U}\right)} D_{10}$ 
We next assume that the capillary entry pressure of the "equivalent well sorted sand" is a good estimate of the capillary entry pressure for the poorly sorted sediment. That is, Eq. (22) is used with $r_{a v g}=r_{e q}$ to calculate the capillary entry pressure of sediment.

\section{Application to Mount Elbert Well}

The model was applied to field data from Mount Elbert gas hydrate stratigraphic test well. As discussed earlier, Mount Elbert well indicates two zones of large gas hydrate saturation (the D and C sand Units) in the stratigraphically highest portions of those sands (Boswell et al., 2011). High resolution grain size distribution data are also available from the well (Winters et al., 2011). In addition, as mentioned earlier, researchers have suggested that such hydrate accumulations are free gas accumulations converted to hydrate after being placed in the GHSZ (Boswell et al., 2011; Collett et al., 2011). Therefore, the Mount Elbert data should be a good test of the proposed model.

Analyzing the grain size distribution in different depths of Mount Elbert well data (Winters et al., 2011) reveals that the coefficient of uniformity for majority of depths fall in the range of $1<U<20$. In addition, the grain sizes mostly fall between $60 \mu \mathrm{m}$ and $600 \mu \mathrm{m}$. Therefore, the Breyer's estimate of permeability should be applicable (Odong, 2008). Interfacial tension between gas and aqueous phases was estimated to be $65 \mathrm{mN} / \mathrm{m}$ at $2{ }^{\circ} \mathrm{C}$ and $6.4 \mathrm{MPa}$ (Jho et al., 1978). Brine salinity in Mount Elbert well is very small, 4 to $11 \mathrm{ppt}$, (Torres et al., 2011) and thus has a density very close to that of fresh water, $1000 \mathrm{~kg} / \mathrm{m}^{3}$. Based on these properties and Eq. (28), the capillary entry pressure versus depth was calculated from grain size distribution at each depth. Profiles of the $10^{\text {th }}$ and $50^{\text {th }}$ percentile of grain size in sand Units D and C are shown in Fig. 1a. The corresponding capillary entry pressure profile is shown as connected dots in Fig. 7 a. 


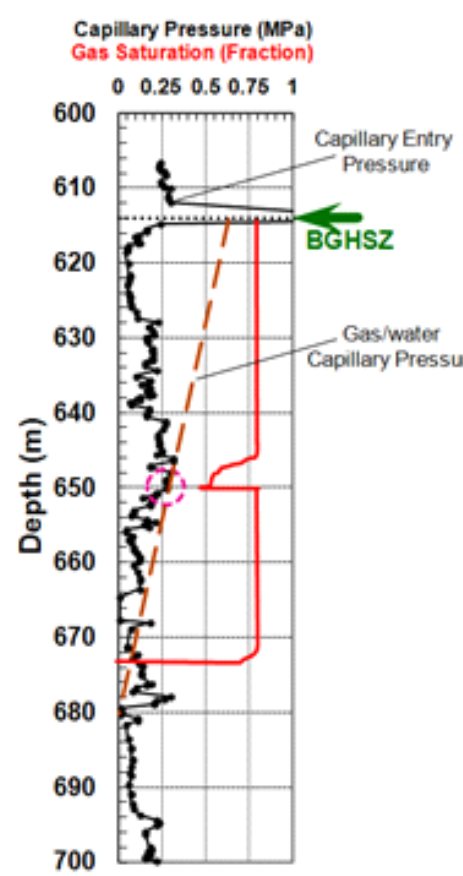

(a)

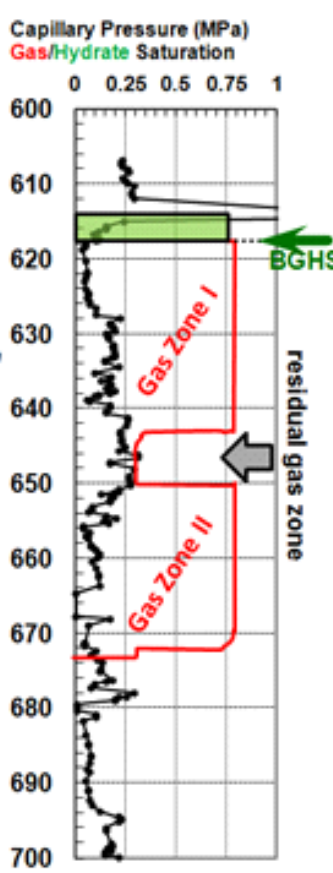

(b)

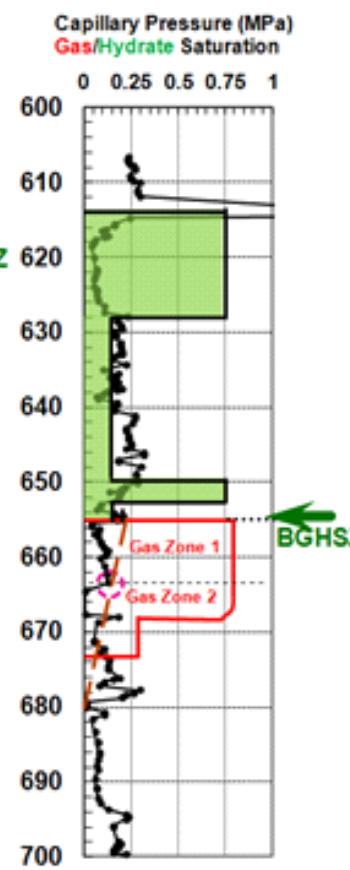

(c)

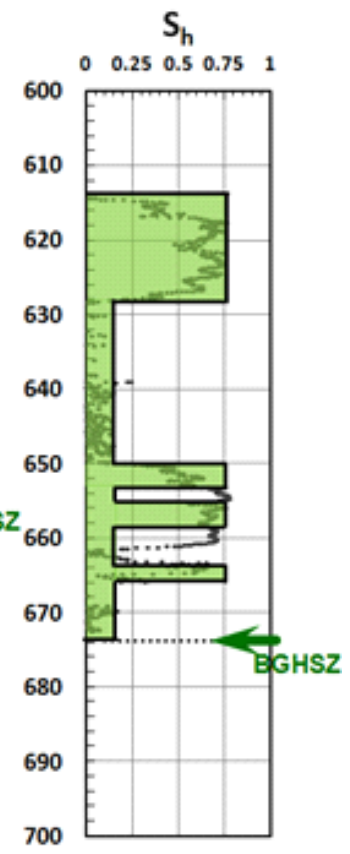

(d)

Fig. 7. (a) Capillary entry pressure, estimated from grain size distribution and Eqs. (22) through (28), versus depth (connected dots) along with estimated initial gas saturation (red solid line) when the BGHSZ was above the zone in which hydrate is currently present. Gas/water capillary pressure along the gas column is shown as dashed line. The gas saturation profile changes as the BGHSZ moves down through the gas column and hydrate forms. (b) The BGHSZ has moved 3.5 meters downward. Gas transported from the lower portion of Unit D and water imbibed from below to create gas zone I. Gas zones I and II are no longer in communication due to the capillary barrier between them (shown with an hollow circle) at $650 \mathrm{~m}$. (c) Gas saturation profile (red) when BGHSZ has moved downward through the gas column to a depth of $655 \mathrm{~m}$ and the resulting hydrate saturation (green fill). Indicated gas zones 1 and 2 are no longer communicating due to the capillary barrier (shown with an hollow circle) at $664 \mathrm{~m}$. (d) Final hydrate saturation profile (using $R_{v}=0.55$ ) after BGHSZ has moved below the bottom of gas column. The log derived hydrate saturations (Lee and Collett, 2011) are shown as dots.

We assume that the gas accumulated in the depth interval between $614 \mathrm{~m}$ and $673 \mathrm{~m}$ (i.e. within sand Units D and C) prior to the descent of BGHSZ. The estimated capillary entry pressure at depth $614 \mathrm{~m}$ (Fig. 7a) indicates that such long free gas column could be maintained by the sealing lithology at this depth. Structural traps would have also augmented such conventional gas traps as Boswell et al. (2011) describe the accumulations in Mount Elbert well to be trapped by at least one western bounding fault and a four-way fold closure. It is also assumed that the accumulation was no longer connected to the source of charge. Figure 7 a shows the estimated gas saturation profile (red solid line) in the gas reservoir prior to its conversion to hydrate. The profile is obtained from the capillarity characteristics of the host sediment and the presumed depth of the original GWC. The latter determines $P_{c}(z)$ the gas-water capillary pressure profile (dashed line in Fig. 7a), which in combination with the estimated capillary entry pressure determines the initial profile of $\mathrm{S}_{\mathrm{g}}$, similar to that explained in Fig. 2. For most of the gas column, gas saturation was estimated to be very large, close to $1-S_{w, i r r}$; which was then approximated by $1-S_{w, i r r}$ for simplicity. The smaller gas saturations between $646 \mathrm{~m}$ and $650 \mathrm{~m}$ are due to the fact that the capillary pressure is only slightly larger than the capillary entry 
pressure in that interval. The irreducible water saturation, $S_{w, i r r}$, is assumed to be $20 \%$ for the entire column (Behseresht et al., 2009). Imbibition, i.e. aqueous phase displacing gas phase, does occur when hydrate begins to form, forcing gas phase to move upward to the hydrate stability zone. The residual gas saturation at the end of the imbibition, $S_{g r}$, is assumed to be $30 \%$ (Peng et al., 2009).

This long column of gas was supported beneath the clay-rich layer observed at depth of 614 $\mathrm{m}$ in Fig. 7a. In the initial state the gas column is barely connected because the capillary pressure is very close to the capillary entry pressure value at the base of Unit D (depth $650 \mathrm{~m}$, indicated with a hollow circle).

The final input for the model is the value of $R_{v}$. For this illustration we set $R_{v}=0.55$ throughout the sediment. As described below this value makes physical sense and also gives a good match of observed hydrate saturation in Mount Elbert well. The effect of different values of $R_{v}$ will be discussed in a later section.

As the BGHSZ moves downward into the gas column the GWC rises, the gas-water capillary pressure is reduced along the gas column, and the gas saturation decreases to residual saturation below the new position of the GWC. Crucially, the gas-water capillary pressure falls below the capillary entry pressure of the sediment at $650 \mathrm{~m}$. Because the initial capillary pressure at $650 \mathrm{~m}$ is so close to the capillary entry pressure, this happens with the first increment of hydrate formation. Therefore, the originally connected gas column starts acting as two noncommunicating columns of gas almost immediately after hydrate starts forming. Thus gas that migrates to the GHSZ in Unit D comes only from within Unit D itself. Fig. 7b shows an intermediate step when the BGHSZ is at a depth of about $618 \mathrm{~m}$. Note that gas moving to fill the void due to hydrate formation now rises from the bottom of the upper portion of the accumulation, establishing a residual gas saturation between $644 \mathrm{~m}$ and $650 \mathrm{~m}$. Water must imbibe into the base of Unit D to replace the migrated gas in this 1-D model.

Figure 7c illustrates the situation after the BGHSZ has descended to a depth of about $655 \mathrm{~m}$. In addition to the initial gas saturation between $650 \mathrm{~m}$ and $655 \mathrm{~m}$, some gas from the lower portion of Unit $\mathrm{C}$ has been used to form hydrate in the top section of this unit. For $R_{v}=0.55$ the resulting hydrate saturation is 0.75 (cf. Fig. 4). The capillary pressure profile intersects the capillary entry pressure profile at a depth of $665 \mathrm{~m}$. Thus the remaining gas column comprises two disconnected regions labeled gas zone 1 and gas zone 2 (Fig. 7c). As the BGHSZ keeps moving downward water imbibes from below into the lower part of gas zone 1 to compensate for the volume of gas that moves into the top section of gas zone 1 during hydrate formation. Meanwhile, gas zone 2 saturations remain essentially unchanged from their initial profile, because the gas zone 1 was disconnected from gas zone 2 prior to hydrate formation in gas zone 1.

The hydrate saturation profile once the BGHSZ has moved to the bottom of the gas column (a depth of about $673 \mathrm{~m}$ ) is shown in Fig. 7d. Our prediction is shown as green area and the logderived hydrate saturations are shown as dots. In the imbibed portions of the gas zones (e.g. 629 $\mathrm{m}$ to $650 \mathrm{~m}$ in Unit D), $R_{v}$ is zero; therefore, when the BGHSZ descends into these portions, only aqueous phase moves to fill the void space that accompanies hydrate formation. For $S_{g r}=0.3$, Fig. 4 (curve for $S_{w, i}=0.7$ evaluated at $R_{v}=0$ ) yields a hydrate saturation $S_{h}=0.13$. Thus the model predicts large hydrate saturations of 0.75 in the upper portions of Units $\mathrm{C}$ and $\mathrm{D}$, and small hydrate saturations of 0.13 in the lower portions. 


\section{Comparing the Model Prediction with Log-Derived Observations}

In Unit $\mathrm{C}$ in Mount Elbert well the zone of major hydrate saturation, which is located at the top of the formation, shows a considerably lower reservoir quality than that of the bottom adjacent part of the formation, bearing little or no hydrate saturation. The situation is similar in Unit D. A gradual decrease in reservoir quality but an abrupt reduction in $S_{h}$ is observed in Unit $\mathrm{D}$ at around $629 \mathrm{~m}$. Though a small reduction in porosity or a small increase in shale content can lead to a significant reduction in permeability (Winters et al., 2011), nevertheless Boswell et al. (2011) consider the lower portion of Unit D (below 629m) to possess sufficient quality to have held free gas. Furthermore, although the estimated capillary entry pressure values in the lower part of Unit D are significantly larger than those in the upper, above 629m, part of the unit (Fig. 7a) they are still smaller than the gas-water capillary pressure along the gas column. Therefore, major part of the lower D Unit seems to have small enough pore entry pressures to hold large free gas saturations, yet contains much less hydrate compared to that in the upper D Unit (Fig. 1).

The model predicts a uniform, small saturation of about $13 \%$ hydrate in the depth ranges of 628-650 and 666-674 meters. The prediction is consistent with observations. The log-derived hydrate saturation is small or zero in the same intervals, while pore fluid geochemical analysis based on dissolved chloride (Torres et al., 2011) indicated the persistence of small values (10\%$20 \%$ ) of hydrate saturations in these depth ranges. The difference in hydrate saturation estimated from $\log$ interpretations and from geochemical analysis is attributed by Torres et al. (2011) to the effect of host sediment on parameterization of the saturation estimates. Lee and Collett (2011) argue that geochemically estimated hydrate saturations in Mount Elbert well may be quantitatively subject to error, as determining baseline salinity is complicated. Nevertheless, they emphasize that salinity data can certainly be used as a qualitative indicator of the presence of hydrates (Lee and Collett, 2011).

Assuming $R_{v}=0.55$ yields a very good match with the log-derived hydrate saturation profile in Unit D. The model correctly predicts the break in hydrate saturation within Unit $\mathrm{C}$ at $663 \mathrm{~m}$, but underestimates the thickness of the large hydrate saturation portion in Unit C between $650 \mathrm{~m}$ and $660 \mathrm{~m}$. The mass of methane in the hydrate exceeds the mass of methane assumed in the initial gas accumulation in Unit C (Fig. 7a). As discussed in Section 4.4, increasing the initial gas charge does not change the predicted profile in Unit $\mathrm{C}$. Thus it appears that more gas migrated into Unit $\mathrm{C}$ during hydrate conversion. One possibility is that the gas charge resumed as the BGHSZ descended, perhaps triggered by the large-scale fluid migration induced by hydrate formation. Another possible factor is the wider lateral extent of Unit $\mathrm{C}$ hydrate compared to Unit D. This could have enabled along-dip gas flow to contribute gas to the hydrate-bearing interval in Unit $\mathrm{C}$ but not in Unit D. This contribution is neglected in the 1D model, which thus provides a lower bound on the hydrate thickness.

\section{Effect of $R_{v}$ on Hydrate Saturation Profile}

The model presented here imposes no a priori constraint on the value of $R_{v}$ in the upper portions of Units $\mathrm{C}$ and D. It is of interest then to examine the effect of this parameter on the results. The value of $R_{v, \text { stoich }}$ for the Mount Elbert well case, with $S_{g, i} \approx 0.8$, is about 0.69 (see Fig. 4); therefore, for $R_{v}>0.69$ the final state will be hydrate and gas phase only. At Mount Elbert well hydrate and water only are observed, (Lee and Collett, 2011) and thus we should have had $R_{v}<R_{v, \text { stoich }}$. Our model requires the GWC to move upward and gas-water capillary pressure along the gas column to decrease during hydrate formation. A consequence of this 
decrease in capillary pressure is that some imbibition must occur throughout the gas zone, i.e. the aqueous phase saturation $\mathrm{S}_{\mathrm{w}}$ must increase. In this case the value of $R_{v}$ must be less than $R_{v, 1: 1}$ (the ratio at which $S_{\mathrm{w}}$ does not change from its initial value). For temperature and pressure conditions of the depth interval of interest, $R_{v, 1: 1}$ is about 0.59 . This expectation is consistent with the value of $R_{v}=0.55$ that gave a good match in Unit D (Fig. 7).

As $R_{v}$ decreases, less gas enters and the final hydrate saturation decreases (see Fig. 4). Regardless of $R_{v}$ the model converts the entire initial mass of methane in the gas column into hydrate. Thus, smaller $R_{v}$ leads to greater thicknesses of hydrate at smaller saturations in the upper portion of each unit and correspondingly thinner intervals in which residual gas has been converted to hydrate. Figure 8 shows the hydrate saturation profile for $R_{v}$ values of 0 (no gas movement at all), 0.23 and 0.48, along with the log-derived hydrate saturations in Mount Elbert well. Comparison with Fig. $7 \mathrm{~d}$ suggests that while $R_{v}=0.55$ is consistent with hydrate saturation profile in Unit $\mathrm{D}, R_{v}$ may have been smaller, i.e. some value between 0.23 and 0.48 which are shown in Fig. 8, as BGHSZ passed through Unit C. It can be shown that $R_{v}$ is controlled not only by the relative mobilities of gaseous and aqueous phases and capillarity properties of the host sediment but also by the rate of hydrate formation. Therefore, value of $R_{v}$ is expected to convey useful information about the multi-phase flow properties of host sediment, as well as the rate of hydrate formation. These issues will be discussed in detail in a future publication. 


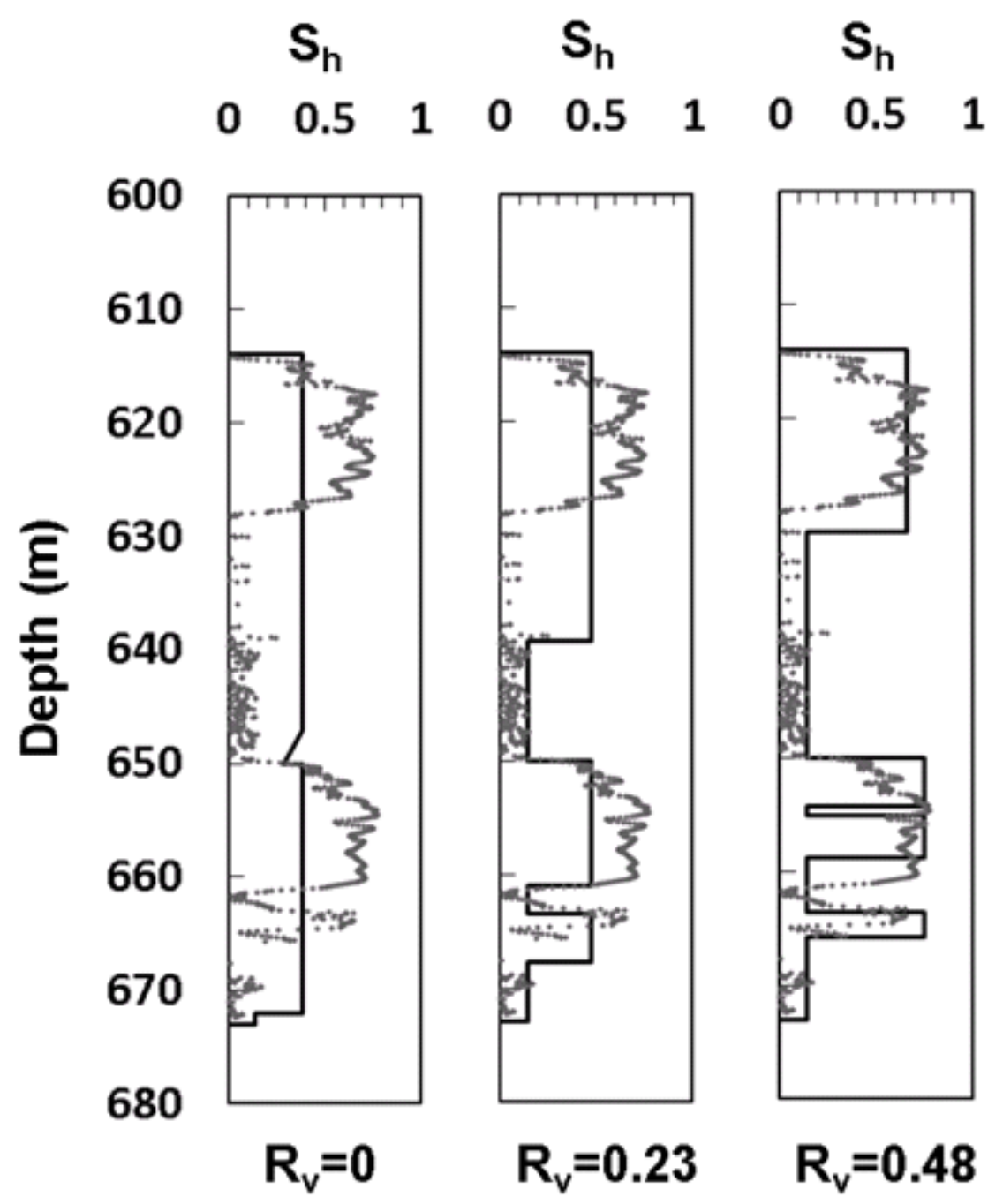

Fig. 8. Estimated hydrate saturation profile for three values of $R_{v}$ along with log-derived hydratesaturation data from Mount Elbert well (Lee and Collett, 2011). Note that mentioned values of $R_{v}$ are used for non-residual gas saturations, forming the major hydrate saturations. For hydrate formation within imbibed intervals, i.e. where the gas saturation has decreased to its residual phase value (e.g. between $630 \mathrm{~m}$ and $650 \mathrm{~m}$ in the panel for $R_{v}=0.48$ ), no gas flow is possible and $R_{v}=0$ is used in those intervals.

\section{Volume of Fluid Transported into the Hydrate-Bearing Zone}

The total transported pore volumes of fluid change linearly with the final hydrate saturation, Eq. (10), with a slope of $K_{\text {trans }} \approx 1.9$ for the assumed $P$ and $T$ within the depth interval of interest in Mount Elbert well. Eq. (10) shows that the amount of fluid transported into the hydrate-bearing zone is substantial, around 1.4 pore volumes in upper portions of units $\mathrm{C}$ and $\mathrm{D}$ in Mount Elbert well (Fig. 9). Clearly such volume changes could not be accommodated by compaction of the sediment. In the lower portions of each unit, $0.3 \mathrm{PV}$ of aqueous phase would have moved into the GHSZ to meet the stoichiometric requirement of converting gas to hydrate. In addition, $0.5 \mathrm{PV}$ of aqueous phase, the difference between $S_{g, i}$ and $S_{g r}\left(\Delta V_{w, d}^{G W C}\right.$ shown in dark blue in Fig. 9) had to enter to replace the gas transported from the imbibed zones (lower 
portion of each disconnected gas sub-column). We will report in future on mechanisms by which this fluid movement could have occurred. We will show that capillary dominated, rather than pressure-driven viscous dominated, flow, is key to the required phase flow for converting a free gas accumulation, with large initial gas saturation, to large hydrate-saturation accumulations. This would also have useful implications in determining the time-scale over which such hydrate accumulations are formed. The magnitude of fluid phase transported is also important in hydratedissociation studies, e.g. when the BGHSZ rises through an existing hydrate accumulation, and pressure build-up evaluation when hydrate dissociates, because the same volume of fluid must be exported from the sediment.

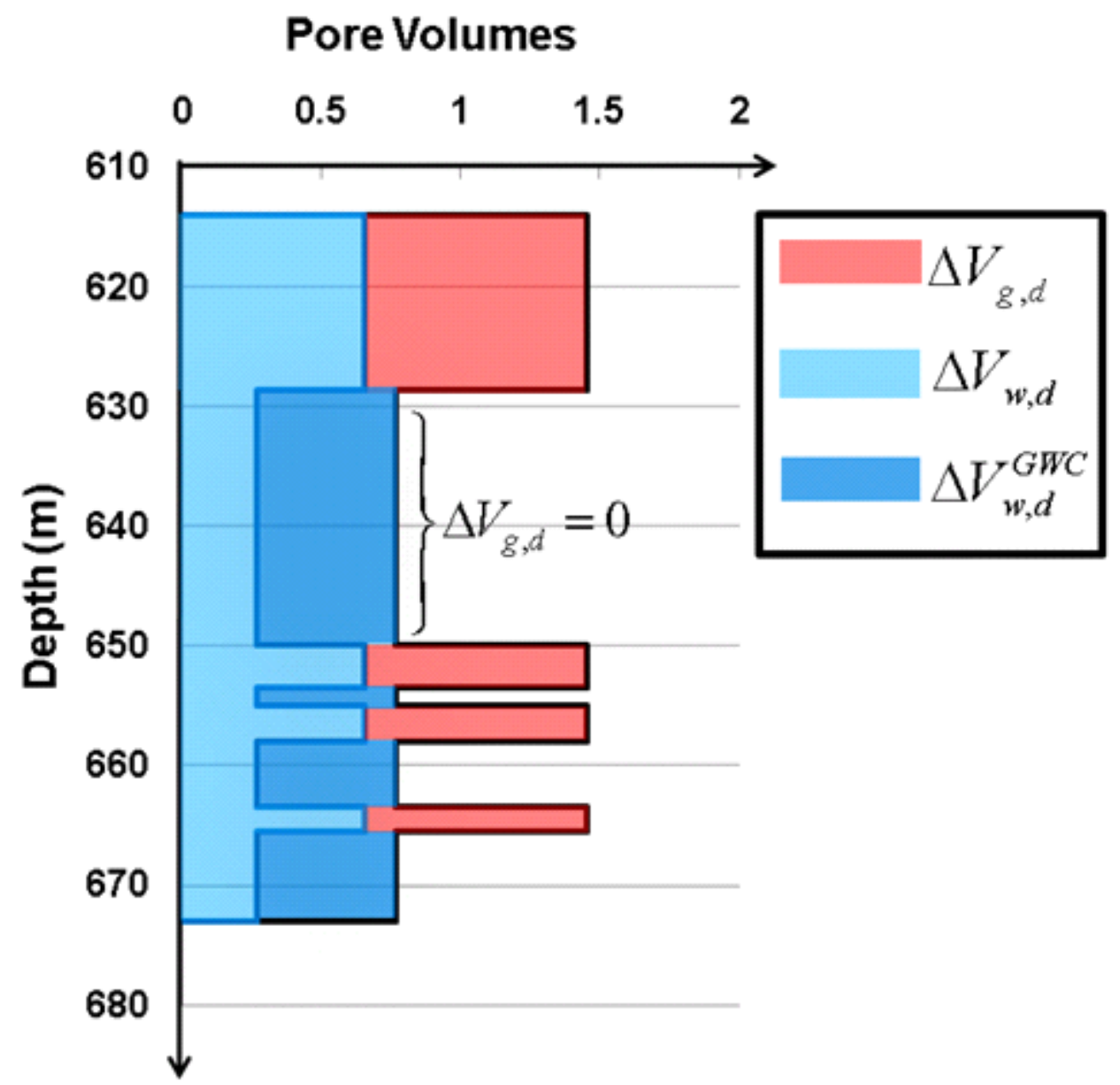

Fig. 9. The model predicts that considerable volumes of fluid (red: gas phase to GHSZ from deeper in the gas column; dark blue: aqueous phase to replace migrated gas; light blue: aqueous phase into GHSZ) have been transported during hydrate formation in Mount Elbert well. The upper portion of each unit accommodated a total of 1.4 pore volumes of gas and aqueous phases. This volume is in addition to the one pore volume initially filled with aqueous and gas phase.

\section{Effect of the Extent of Initial Gas Charge on Modern Hydrate Saturation Distribution}

We now consider two other initial gas saturation profiles: a) a partial charge that resulted in slightly thinner and consequently noncommunicating gas accumulations in Units D and C (Fig. $10 \mathrm{a}-1)$ and $\mathrm{b}$ ) a greater charge that resulted in an initially continuous gas accumulation spanning Units $\mathrm{D}$ and $\mathrm{C}$ beyond the modern hydrate-bearing interval (Fig. 10b-1). 
Figure 10a-1 shows the initial gas saturation profiles within Units $\mathrm{C}$ and $\mathrm{D}$. The column height above the GWC at $668 \mathrm{~m}$ in Unit $\mathrm{C}$ does not provide enough capillary pressure to breach the seal at the base of Unit D. Thus the gas accumulations in zones I and II are noncommunicating. Furthermore, gas only partially fills the Unit D sand because the effective GWC is at the base of Unit D, and the capillary pressure thus does not exceed the capillary entry pressure at $640 \mathrm{~m}$. Figure $10 \mathrm{a}-2$ shows the hydrate saturation profile after converting the gas accumulations (Fig. 10a-1) into hydrate. Much less hydrate forms in Unit D since much less gas is initially present. The model fails to predict the zones of minor hydrate saturation above the base of Unit D (between $640 \mathrm{~m}$ and $650 \mathrm{~m}$ ) and between $668 \mathrm{~m}$ and $673 \mathrm{~m}$ in Unit C.

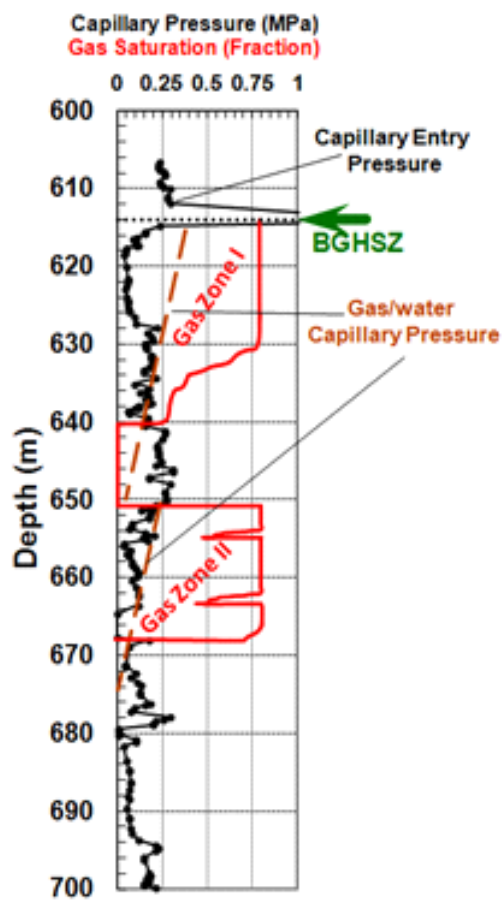

$(a-1)$

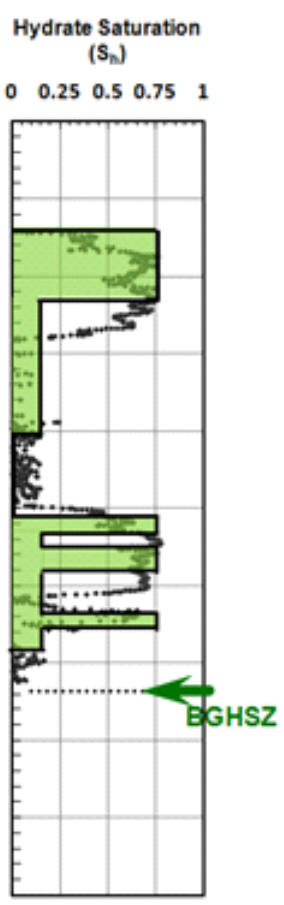

$(\mathrm{a}-2)$

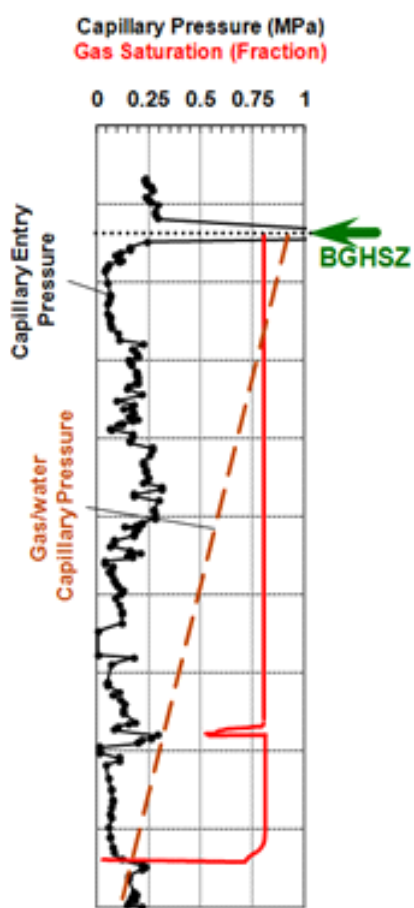

(b-1)

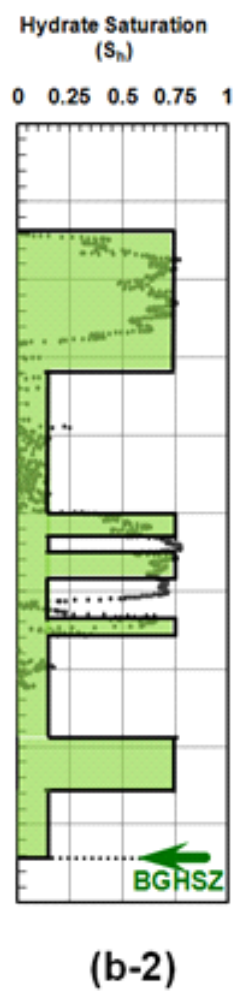

(b-2)

Fig. 10. Effect of initial gas charge on the resulting hydrate saturation profile: (a-1) shows two noncommunicating gas accumulations within the $\mathrm{C}$ and D Units of Mount Elbert well; (a-2) is the resulting hydrate accumulation after converting the gas accumulations in (a-1) into hydrate. Note that the hydrate-bearing interval in both Units C and D are underestimated. (b-1) shows a single long gas accumulation spanning through Unit D and part of Unit C when the BGHSZ is at the top of Unit D. In contrast to (a-1), the gas phase is connected between the units. The resulting hydrate accumulation (b-2) after converting this long gas column into hydrate exhibits the same profile in the upper portion of Unit C (650 $\mathrm{m}$ to $670 \mathrm{~m})$, indicating initial gas charge has little effect on the behavior. Conversion also yields a large hydrate-bearing interval below $678 \mathrm{~m}$ which is not observed in the logs. The hydrate-bearing interval in Unit D is overestimated, because the continuous gas column allows a significant amount of gas to migrate from Unit C into Unit D.

Figure 10b-2 shows the hydrate saturation profile after converting a thicker initial column of gas (Fig. 10b-1) for which the GWC is at $694 \mathrm{~m}$. In this case the hydrate saturation in Unit D is overestimated, because substantial gas moves into Unit $\mathrm{D}$ from Unit $\mathrm{C}$ before the two units are disconnected. The predicted profile between $650 \mathrm{~m}$ and $673 \mathrm{~m}$ is similar to that shown in Fig. $7 \mathrm{~d}$. This is because the gas column below $678 \mathrm{~m}$ becomes disconnected from the gas above that 
point as the GWC rises. This creates a second effective GWC at $678 \mathrm{~m}$, and when this secondary GWC rises to $673 \mathrm{~m}$, the gas within Unit D becomes disconnected from that in Unit C just as in Fig. 7. Thus the absence of hydrate in the good quality sand below $665 \mathrm{~m}$ in Unit $\mathrm{C}$ is not necessarily an indication of insufficient original gas charge. The eventual conversion of the gas below $678 \mathrm{~m}$ yields another major hydrate-bearing interval, below $678 \mathrm{~m}$, which is not observed in Mount Elbert C Unit (Lee and Collett, 2011) in carefully retrieved cores (Rose et al., 2011).

This analysis shows that the depth interval over which modern hydrate saturation is observed is expected to reflect the depth interval over which the original gas reservoir was established. That is why in the calculation above we tacitly assumed the initial gas column height was controlled by other factors (e.g. insufficient charge, a spill point elsewhere on the structure, the connection with the source simply happened to occur at $673 \mathrm{~m}$, etc.). The proposed model predicts gas hydrate columns with the same length as the original gas column but with a very different shape of saturation profile.

\section{Summary of Influence of Grain Size Variation and Phase Volume Changes on Hydrate Saturation Profiles}

A 1-D model was proposed to predict/explain the hydrate saturation profiles in "converted free gas" hydrate reservoirs considering three key elements: (i) volume change during hydrate formation within an existing gas accumulation, (ii) the descent of the base of GHSZ through the accumulation, and (iii) sedimentological variation with depth that causes variation in capillary entry pressure. Applied to a gas accumulation no longer connected to the charge, these elements combine to drive gas migration within the accumulation to the GHSZ. Substantial aqueous phase migration to and through the sediment column to the GHSZ accompanies the gas migration and hydrate formation. This leads to large hydrate saturations in the upper portion of the column and small saturations in the lower portion, even if the lower portion is sand-rich. Internal gas migration raises the gas/water contact, raising the possibility of disconnection of the remaining gas column at layers having relatively large capillary entry pressure. When this occurs, the disconnected subcolumns also yield large and small hydrate saturations in their upper and lower portions, respectively. The hydrate saturation profile resulting from these processes is nonuniform with rather sharp basal contacts, i.e. large $S_{h}$ values interspersed between small values, even if the initial gas saturation profile is uniform. The nonuniformity cannot be explained via association of major hydrate saturations with better quality sands.

The model was applied on data from Mount Elbert well located in one of the methane hydrate reservoirs in Alaskan North Slope. For a physically reasonable choice of the relative volumes of gas and aqueous phases that enter the hydrate zone, the model predictions match the field data satisfactorily. Of particular note, the model accounts for a twenty-meter interval of small hydrate saturation in fairly sand-rich sediment between two accumulations of large hydrate saturation. It also explains how in Unit $\mathrm{C}$ the major methane hydrate accumulation is at the top lower-quality sand but not in the lower better-quality deposit, even if the latter sand is originally charged with gas.

Material balance analysis shows that a considerable amount of fluid (of order 1 pore volume of gaseous and aqueous phases) needs to be transported during hydrate formation. In the 1D model analyzed here, all transport is vertical. This maximizes the influence of sedimentological control on the final hydrate distribution. As the areal extent of the gas accumulation increases or the dip of the structure increases, the contribution of along-dip flow to the required gas transport 
increases, and the 1D model yields a lower bound on the thickness of the intervals with large hydrate saturation.

\section{Gas Reservoir Conversion to Hydrate: Role of Pore-Scale Imbibition}

The preceding sections have laid out the main physical phenomena that drive the conversion of a gas reservoir into a hydrate-bearing formation. We have assumed that during conversion the gas reservoir is no longer connected to the source of gas charge, and consequently the conversion of gas to hydrate causes the capillary pressure in the gas accumulation to decrease. We are thus led to consider in more detail the fluid phase displacement that must accompany this decrease in capillary pressure.

Viewed from the pore scale, conversion of a gas reservoir to hydrate is an imbibition process. Any reduction in capillary pressure will cause the aqueous phase to imbibe into the gas-saturated portion of the formation. In the gas column below the base of the gas hydrate stability zone, this imbibition behaves classically. That is, the gas saturation decreases, following the characteristic $P_{c}-S_{w}$ curve for the sediment, and the gas-water contact at the base of the gas column rises. Imbibition at or within the hydrate stability zone introduces a novel aspect, however: the growth of hydrate at the gas/water interface makes that interface rigid. Because of the mechanical strength associated with the hydrate skin, displacing such an interface would in principle require a pressure difference over and above the capillary pressure needed to displace the geometrically equivalent fluid/fluid interface. We do not explicitly account for this mechanical strength in this work, not least because no measurements are available that would enable estimating its value. Instead, and more fundamentally, we propose in this research that the hydrate skin is likely to act as a permeable membrane, not as an impermeable barrier. Thus the aqueous phase can filter through the skin in response to a reduction in gas phase pressure.

This conceptual model leads to two useful limiting cases. In one limit, we assume water filters through the hydrate skin slowly, or, equivalently, we assume water moves very slowly from some large external source to the gas-hydrate-water interfaces. The slow arrival of water could be because of small permeability of the sediment through which the aqueous phase is transported, or because the driving force (saturation gradient or potential gradient or both) is small. The definition of "slow" is not absolute; the relevant measure is whether the rate of water supply is slow relative to the rate of hydrate formation. If so, then all water arriving at the gashydrate-water interface will be immediately incorporated into the hydrate phase, growing into the volume originally occupied by the gas phase.

In the other limiting case we assume that water filters through the hydrate skin rapidly, or equivalently that the rate of water supply is fast compared to the rate of hydrate growth. In this case, the gas/water interface is continually renewed. Thus it is possible for a traditional imbibition event to occur, in which two gas/water interfaces merge and enable gas to withdraw spontaneously from a pore as water fills the pore. Since we assume hydrate grows into the gas phase from a gas/water interface, the water in the newly imbibed pore will not be incorporated into the hydrate phase. Hydrate accumulates at an interface as long as the interface moves only incrementally in response to reductions in capillary pressure.

These two limiting cases should bracket the spectrum of behavior observed in nature. The remainder of this section explores in more detail the implications of this pore-scale perspective on hydrate growth. 


\section{Hydrate Formation and Growth in Pore Scale}

It has been proved that hydrate formation can take place either from single phase (gassaturated aqueous phase) or two phases (gaseous and aqueous phases) (Tohidi et al., 2001; Katsuki et al., 2006, 2007; Ohmura et al., 2005; Teng et al., 1995; Shindo et al., 1995). However, forming hydrate from a single phase system is unlikely to obtain a high hydrate saturation, because of the low solubility of methane in aqueous phase (Handa, 1990). Thus for reservoirs in arctic regions where $>70 \%$ hydrate saturation is often observed, a two-phase system is required.

In the porous medium, the first nucleation of hydrate crystal occurs in low energy regions. In a two-phase system, one possible nucleation site is along the interface between gas and water. Later hydrate could possibly grow into either gaseous or aqueous phase. It seems the growth direction is controlled by the availability of gas or water. That is, if gas is limited, hydrate grows into the gaseous phase (Tohidi et al., 2001; Katsuki et al., 2007), while the limitation of water would let hydrate grow into the aqueous phase (Ohmura et al., 2005; Dong Lee et al., 2005). Another possible hydrate nucleation site is on the grain surface (Katsuki et al., 2006). However, the grain surface is either exposed to gas or water, and therefore hydrate formation is restricted due to the lack of the other material.

After the first nucleation, hydrate growth requires the transportation of a material through the hydrate layer (if hydrate grows into gaseous phase, water must migrate through the hydrate layer to the gaseous phase, while growing in the other direction requires gas to migrate through the hydrate layer). Models were proposed to address such mechanism. In these models, micro defects in hydrate are considered as permeable conduits. (Mori and Mochizuki, 1997) suggested that water is able to pass through these conduits due to capillarity. Thus, hydrate formation is able to occur and grow into the gaseous phase. On the other hand, the model proposed by (Hirai et al., 1996) argued that gas molecules can transport through the hydrate layer to the aqueous phase due to diffusion, and thus hydrate is able to grow into gaseous phase. However, the diffusion rate largely limits the hydrate formation rate, and therefore unlikely to capture the physics.

The different experimental observations and model descriptions suggest an on-going scientific debate on the hydrate growth in the porous medium, a reflection of our lack of understanding of the pore-level physics. In this section we examine the implications of the concept that proposed hydrate grows into the gaseous phase, due to the fact that in this work gas is the limiting resource in the reservoir.

This section focuses on pore scale processes. To explain the boundary conditions that will be applied to the pore scale model, we motivate this section with a recapitulation of the bed-scale process. Our field-scale model for the arctic region uses the concept of a petroleum system. Figure 11 shows the conceptual model. The reservoir was originally saturated with water. It was connecting to the deep gas source through faults and fractures. A capillary seal existed on top of the reservoir, and closures were capable of trapping the initial gas in the reservoir (not shown in the figure). BGHSZ originally was above the reservoir at an arbitrary depth. Permafrost extended from the ground surface to a depth that was shallower than BGHSZ. The distance between BGHSZ and the base of permafrost was in the magnitude of several hundred meters, as suggested by (Majorowicz et al., 2008).

Over the geological time, thermogenic gas migrated upwards through these conduits (Fig. 11). Due to the low capillary entry pressure of the sediments and high capillary pressure between gas and water, it is likely that in most parts of the reservoir water was displaced to the irreducible 
value (Peng, 2011). Gas slowly accumulated beneath the capillary seal, expanded downwards and finally filled the reservoir. When gas charge was complete, by assumption the communication between the reservoir and the deep gas source was lost. Therefore it became an isolated system for gas. We assume BGHSZ was still above the reservoir during this period, and thus no hydrate formed even though both gas and water were available.

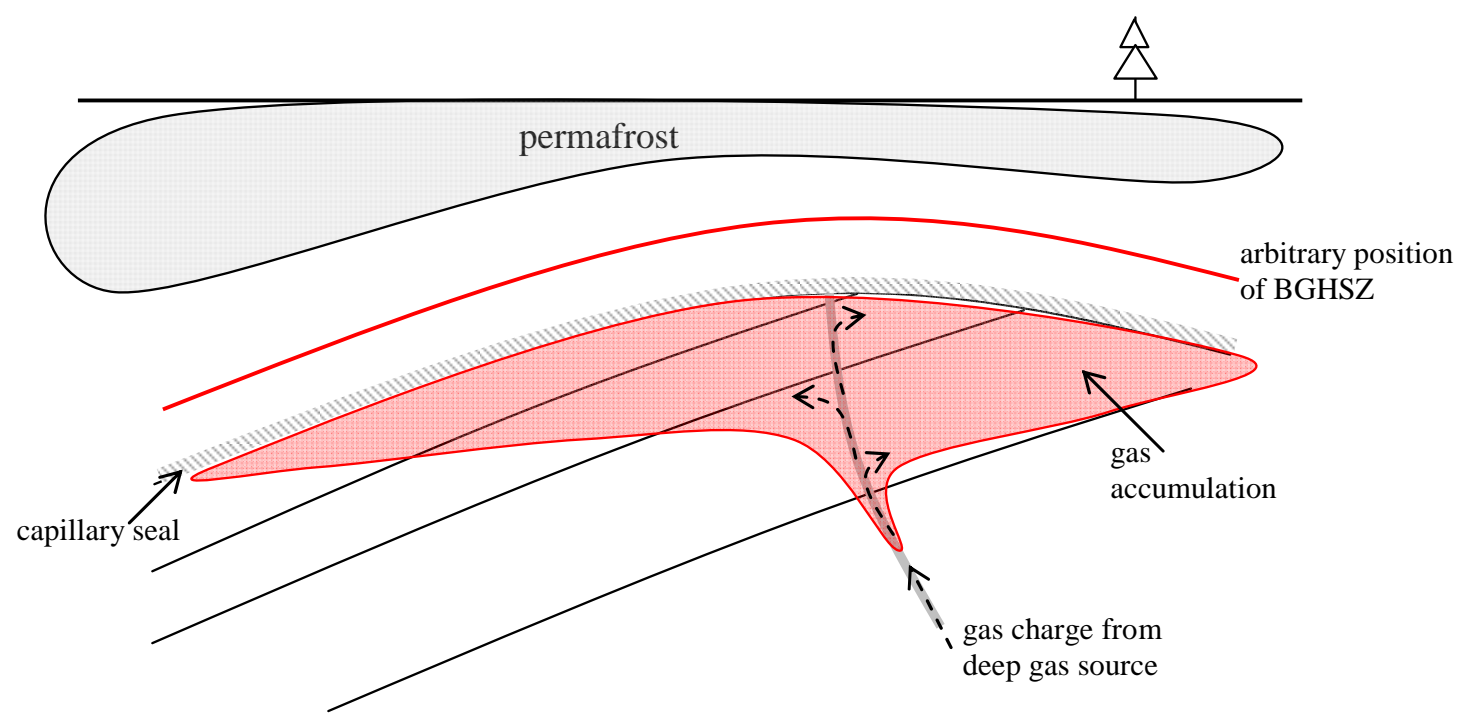

Fig. 11. A schematic of the initial gas charge in the reservoir and the position of permafrost and BGHSZ.

Gas is in the red color.

BGHSZ began to descend when gas charge in the reservoir was complete (Fig. 12). In arctic regions, it is a general trend that BGHSZ has been moving downwards in the past several million years (Majorowicz et al., 2008). When BGHSZ entered the reservoir, gas hydrate formed at BGHSZ. Hydrate formation from a two phase system (gas/water) is controlled by microscopic events, which will be investigated in the pore level (Section 2.1). The density of methane molecules in hydrate is computed by the following equation:

$\rho_{g_{-} \text {hydrate }}=\rho_{h} \frac{M_{g}}{M_{g}+N M_{w}}$

where $\rho_{g \text { hydrate }}$ is gas molecule density in the clathrate. $\rho_{h}$ is hydrate density, which is $910 \mathrm{~kg} / \mathrm{m}^{3}$ (Sloan, 2003). $M_{g}$ and $M_{w}$ are the molecular weight of gas and water, respectively. $\mathrm{N}$ is the hydration number, and in our work it is 5.75. For ideal methane hydrate clathrate Eq. (29) gives $\rho_{g_{\text {h hydrate }}}=122 \mathrm{~kg} / \mathrm{m}^{3}$, which is much larger than the methane density in the reservoirs studied in this work (For example, methane density in Mount Elbert reservoir is $\sim 55 \mathrm{~kg} / \mathrm{m}^{3}$ and in Mallik reservoir is $\sim 90 \mathrm{~kg} / \mathrm{m}^{3}$ ). This result suggests that in the reservoir condition the volume of hydrate is smaller than the volume of gas converted to form hydrate (we assume water is the unlimited phase whose volume change is not considered). Thus the transportation of free methane and water into the local porous medium is required to fill the vacancy that would otherwise be created. The newly-fed gas and water were converted into gas hydrate, and further sucked in more gas and water from other lower reservoir layers. Such positive feedback continued until no more fluid invasion could occur, at which moment the porous medium was only filled by hydrate and water (the coexistence of hydrate and gas was not observed in either reservoir). 


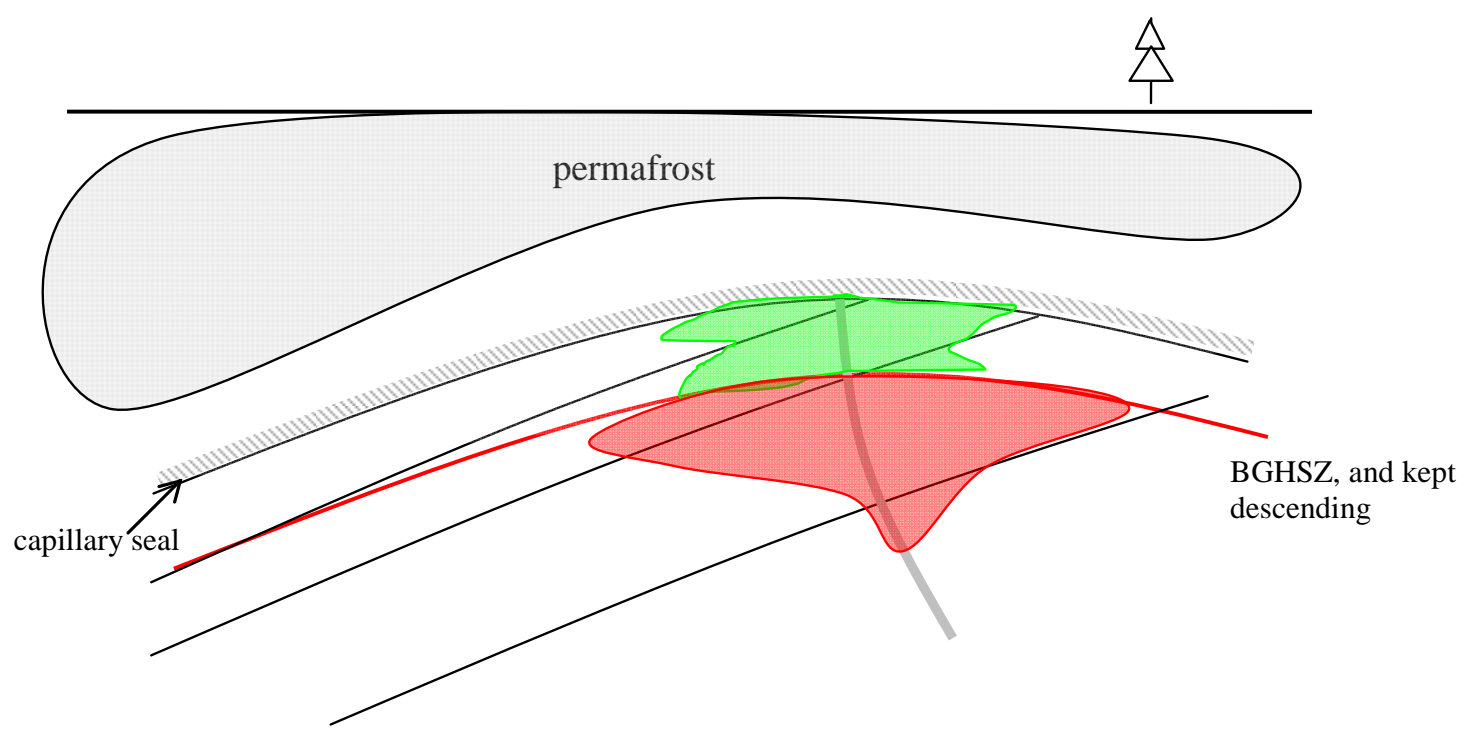

Fig. 12. A schematic of gas and hydrate distribution in the reservoir when BGHSZ was descending. Permafrost also expanded downwards. Gas is in the red color and hydrate is in the green color.

BGHSZ kept descending and converting gas and water into gas hydrate. Hydrate formation was complete when BGHSZ reached the base of the reservoir (the descending of BGHSZ from the top to the base of the reservoir took tens of thousands of years). The final hydrate profile differs from the initial gas/water distribution (Fig. 13). It has a characteristic pattern with two distinct regions of gas hydrate saturation.

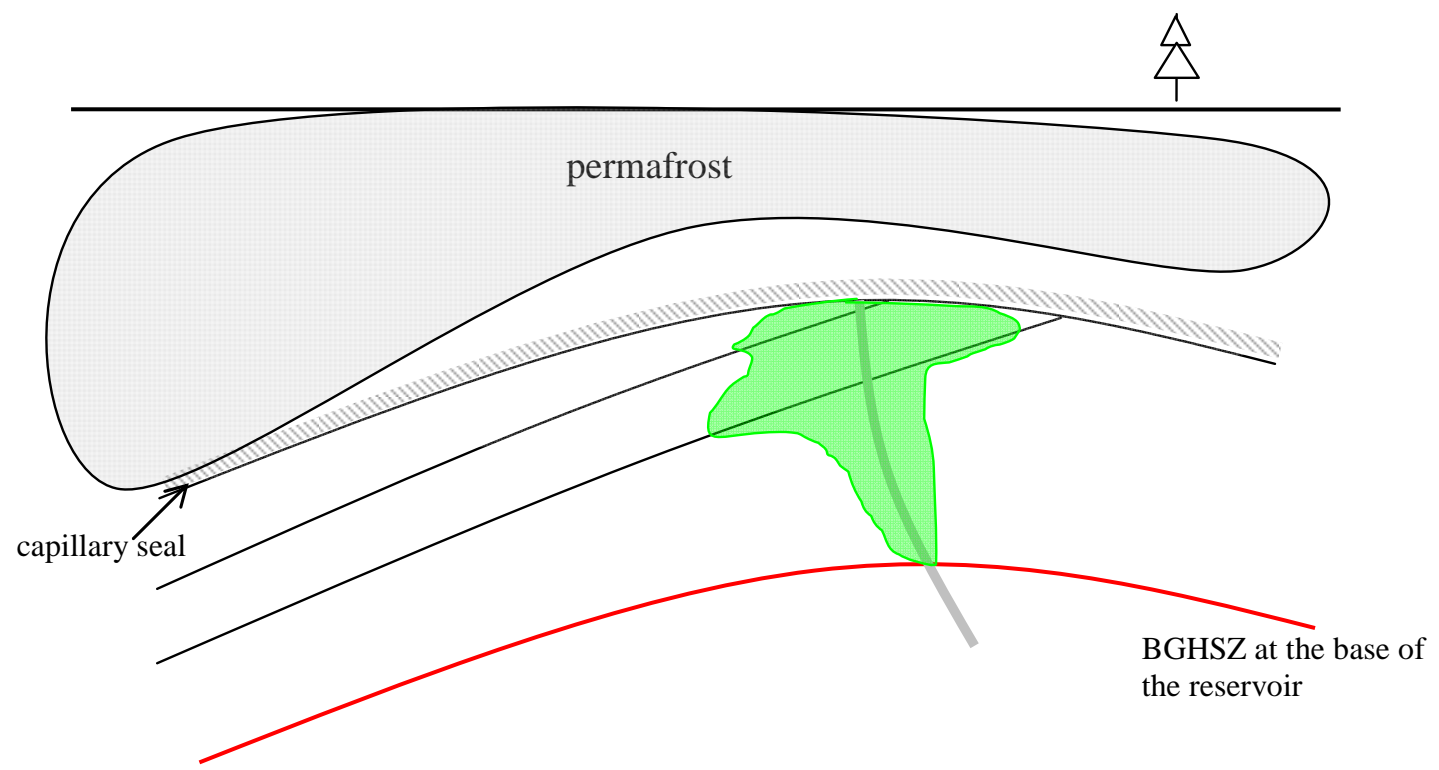

Fig. 13. A schematic of final hydrate distribution in the reservoir when BGHSZ reached the base of the reservoir. Hydrate saturation increases from base to the top of the reservoir. Permafrost expanded further to the deeper depth. 


\section{Scenarios of Hydrate Formation and Growth in Porous Medium}

The initial gas and water distribution in the porous medium is controlled by drainage endpoint, at which gas displaces water to its irreducible value. We assume hydrate formation and growth follow the pathway of conventional imbibition, and thus a 2D simulation by LSMPQS is applied to show the detailed movement of gas/water interface during imbibitions (and also hydrate growth path). LSMPQS is a fundamental numerical technique that is capable of modeling capillarity-dominated process without any geometry simplification, with its accuracy and correctness widely tested against the experimental results (Prodanović and Bryant, 2006; Prodanović et al., 2008). Two different hydrate formation scenarios are proposed by studying the imbibition process.

The hydrate formation and growth in the porous medium uses several general assumptions:

1. The gaseous phase (pure methane) and aqueous phase (water with constant salinity) are at capillary equilibrium, so that gas/water interfaces are static. Our model also assumes both gas and water are connecting to the bulk volume, and thus neither of them would be exhausted (that is, in the pore scale we do not apply the assumption of limiting gas and unlimited water). We consider the case that the salt ion diffusion rate is much higher than hydrate formation rate, and therefore salinity does not inhibit conversion.

2. The first layer of hydrate crystal forms along the interface between methane and water when the local temperature and pressure reach the phase equilibrium boundary (the hydrate-water/gas-water boundary).

3. Forming a layer of hydrate reduces the gaseous phase volume more than the aqueous phase, since volumetrically hydrate formation consumes more methane than water at typical hydrate stability conditions ( $P=5$ to $10 \mathrm{MPa}, T=273$ to $283 \mathrm{~K}$ ). Therefore, hydrate preferentially grows into the gaseous phase. In this fashion a layer of hydrate appears between gas and water, and the gas/water interface is replaced by a methane/hydrate interface, a thin hydrate layer, and a hydrate/water interface.

4. Continued growth of hydrate requires water to migrate from the aqueous phase in the sediment through microscopic defects (e.g. crystal defects within the hydrate, grain surface roughness, etc.) to the methane/hydrate interface.

5. The total vacancy (reduction in methane volume + reduction in water volume increase in hydrate volume) generated when an incremental volume of hydrate forms is filled by aqueous phase. Depending upon the connectivity of the aqueous phase (between the interface where hydrate is forming and the bulk phase of formation water) and, more critically, upon the rate at which water can migrate through the existing hydrate in step (4), the rate of this filling may be faster or slower than the rate of hydrate formation at the interface.

In this model, hydrate saturation varies. If the water supply rate is much smaller than the hydrate formation rate, hydrate saturation will reach a maximum value. On the other hand if the water supply rate is much larger than the hydrate formation rate, hydrate saturation will reach the minimum value. We describe these two limiting scenarios next.

Hydrate Saturation and Distribution in Porous Medium, Scenario A: Water Supply Rate Much Smaller than Hydrate Formation Rate

Figure 14 shows a small 2D domain. It is a simple granular medium with large open spaces. The initial aqueous and gaseous phase distribution (Fig. 14a) is obtained by simulating a 
drainage endpoint using LSMPQS. The initial hydrate nucleation happens on gas (red)/water (blue) interface. The hydrate layer does not form as a single crystal. The microscopic defects within the layer of crystals allow water to imbibe through the hydrate layer and form a thin layer of water on the hydrate surface (Fig. 14b the zoom-in figure, notice the thin water layer (blue) on the gaseous phase (green)). Since hydrate formation is rapid compared to water supply, this water layer is quickly converted into hydrate. This further reduces the gaseous phase pressure (Figs. 14b and c) and water again imbibes onto the gas-hydrate interface. During this process, we assume that the curvature of hydrate layer maintains the curvature of capillary-equilibrium between gas and water, and therefore the hydrate invasion into the gaseous phase can be approximated as the imbibition process (the curves are computed by LSMPQS simulation). This stage of the hydrate formation (Figs. 14b and c) is referred to as stage 1 .

(a) The initial methane (red) and water (blue) distribution in a granular medium (gray disks represent the grains). The distribution is in capillary equilibrium (satisfies Young-Laplace equation), obtained at the drainage endpoint by the LSMPQS simulation. The colors of the interface are only for the demonstration purpose, and should not be associated with the colors of the phases.

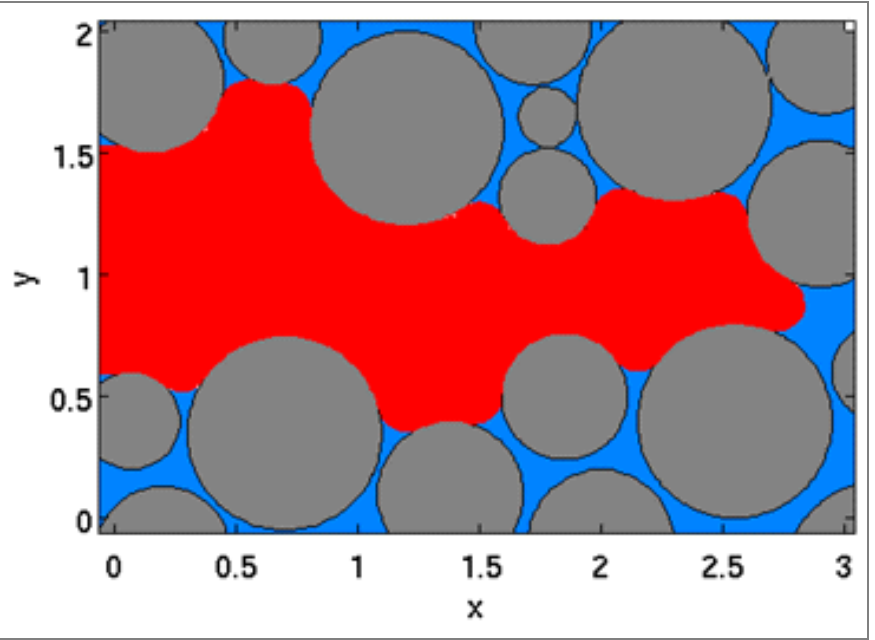

(b) Stage 1, the incremental movements of methane/water interface due to hydrate formation (phases are not colored for a better visualization). This figure shows three sequential representative locations of interfaces during hydrate formation. The arrows indicate the directions that the interface moves and in which hydrate grows. The first layer of hydrate will form at the interface. Subsequent hydrate continues to grow into the gaseous phase. A schematic (inset and zoom in below main figure) describes the mechanism by which water is supplied to maintain hydrate formation at the interface between methane and hydrate. Numerous tortuous conduits exist in the hydrate layer (green) because of the crystal defects. Water (blue) can therefore be sucked through the layer and coats the hydrate surface. When in contact with methane (red), new hydrate can form at the methane/water interface. This is the driving mechanism for hydrate "invasion" of the methane-occupied pores.

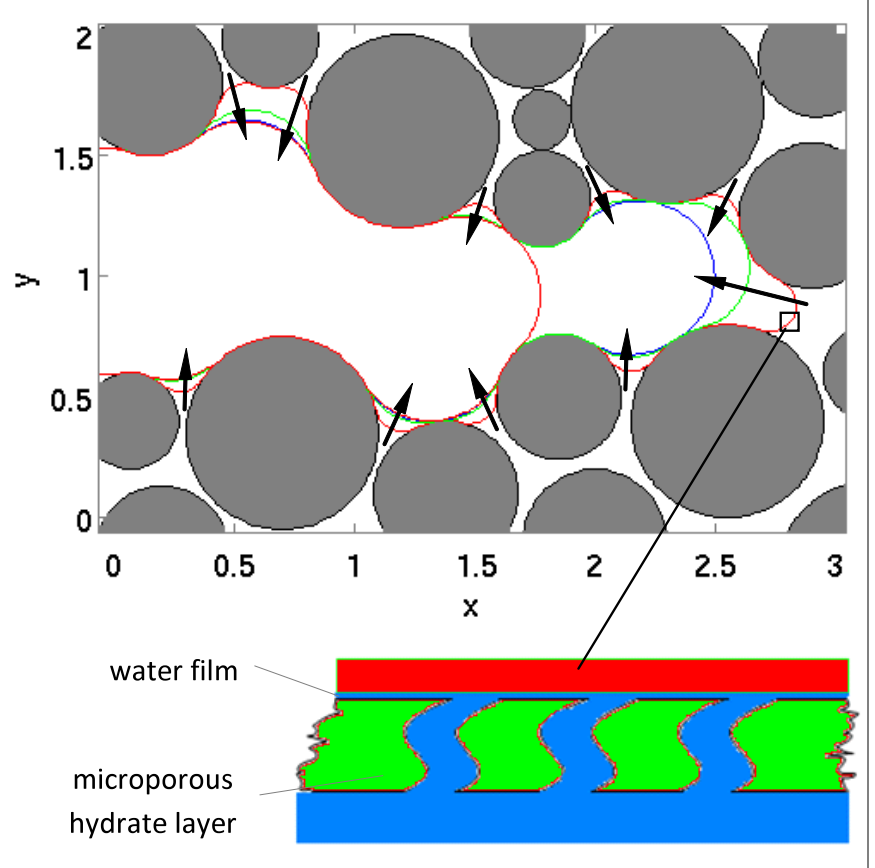


(c) Hydrate distribution (green) when the interface (indicated by the arrow) is at the critical point. From this point, an infinitesimal decrement of capillary pressure will make the interface unstable. (d) Stage 2, the incremental movement of methane-water interface (from green to red interfaces; phases are not shown). The red curve is the capillary-equilibrium curve after an imbibition jump from the position indicated by the green curve, computed as if aqueous phase were displacing gas. Because the scenario considered here assumes that water can be supplied only very slowly, the Melrose jump cannot occur. Instead gradual incremental movement from green to red curves (blue curve is an example intermediate position) happens during this stage. The rapid formation of hydrate means that similar to $\mathrm{B}$, hydrate grows from the water film on the methane/hydrate interface and gradually invades the gaseous phase.

(e) Hydrate distribution (green region) after stage 2 . The total hydrate distribution is due to the hydrate formations in stages 1 and 2 .

Hydrate occupies the entire space that was taken up by methane before. That is, hydrate distribution replaces the initial methane gas distribution.
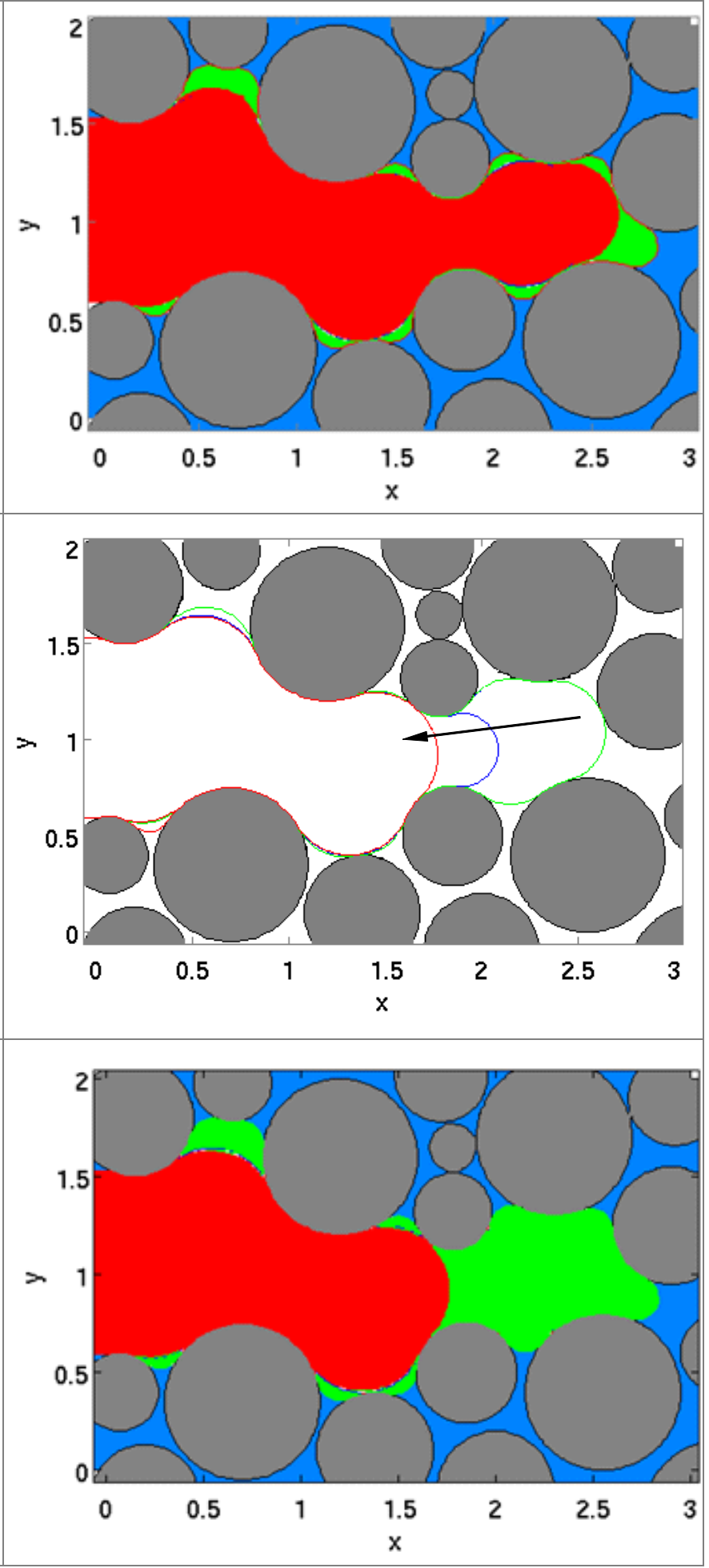
(f) Stages 1 and 2 repeat themselves cyclically, which allows the hydrate formation slowly invades the gaseous phase. The final hydrate distribution from the right figure (green is hydrate and blue is water) is identical with the initial methane distribution in (a).

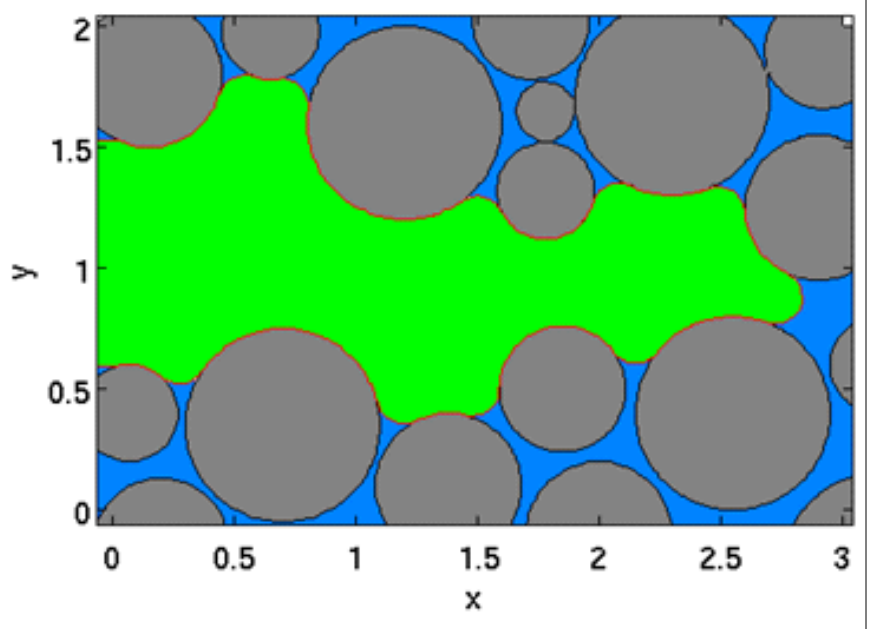

Fig. 14. Schematics of Scenario A that water supply rate is much smaller than hydrate formation rate, which gives the maximum hydrate saturation. The gray disks are sand grains, blue regions correspond to the aqueous phase, green region is hydrate, and the red region is gas phase.

The critical feature of this limiting case is that the gas/hydrate interfaces will gradually advance into the gas phase, so that adjacent interfaces eventually merge. This also means that separated gas/water interfaces merge as well, as water always coats the hydrate surface (Fig. 14b the zoom-in figure) because of capillarity. In classical imbibition, the interface will jump to a new location. This is the Melrose condition for imbibing a pore. However when two gas/hydrate interfaces (also gas/water interfaces) merge, a spontaneous Melrose imbibition event is not possible, because the fluid (water) is converted to a solid (hydrate) as soon as it reaches the gas/hydrate interface, and thus no extra water is available for the imbibition jump. In other words, the limiting water supply disables imbibition events, and only the slow and incremental movement of interface is allowed (Figs. $14 \mathrm{~d}$ and e). This increment movement gives a similar pattern as of Figs. $14 \mathrm{~b}$ and $\mathrm{c}$. That is, water is sucked through the hydrate layer and coats the hydrate surface as a thin layer, of which the water is later converted into hydrate. This gradual movement of hydrate finally allows the interface to move into the following pores. We refer to this (Figs. 14d and e) as stage 2.

This incremental motion has an important implication: all the gas phase initially present is eventually converted to hydrate, and extra gas from other layers is required for the hydrate formation since the initial gas is insufficient. The final hydrate saturation has the same pore-scale "footprint" (occupies the same pores, throats, etc.) as the initial gas saturation (Fig. 14f), and it is the maximum value that can be predicted by this model.

These two stages have the same behavior but driven by different forces. Stage 1 is controlled by the capillary equilibrium, which is independent of the water supply rate. Stage 2, which would exhibit an imbibition jump if both phases at the interface were fluids, happens only because the water supply rate is slower than hydrate formation rate on the methane/hydrate interface. These two stages happen cyclically.

\section{Hydrate Saturation and Distribution in Porous Medium, Scenario B: Water Supply Rate Much Greater than Hydrate Formation Rate}

This scenario has the same stage 1 as scenario A: hydrate slowly invades gaseous phase (Figs. 15b and c). When the gas/water interface reaches the critical point at which two interfaces merge (the end of stage 1), the fluid behavior becomes different from scenario A. The 
assumption that water supply rate is much larger than the hydrate formation rate indicates that water supply is no longer a constraint of imbibition. At the critical point, a Melrose imbibition jump takes place due to the unstable fluid/fluid interface, and invades gas-filled pores in an abrupt fashion.

(a) The initial methane (red) and water (blue) distribution in a porous medium (gray disks represent the sand grains). The distribution is in capillary equilibrium (satisfies Young-Laplace equation), obtained from the drainage endpoint by the LSMPQS simulation. The colors of the interface are only for the demonstration purpose, and should not be associated with the colors of the phases. This is the same as Fig. $14 \mathrm{a}$.

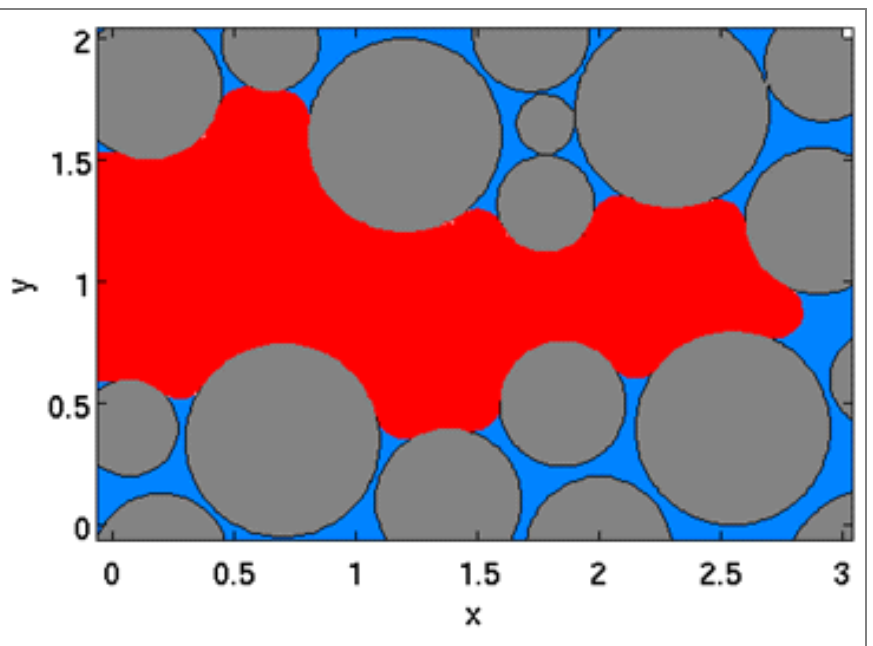

(b) Stage 1, the incremental movements of interface due to hydrate formation (from the red to blue interfaces). The arrow indicates the direction of methane/water interface movement and hydrate growth. The first layer of hydrate forms on the original methane/water interface. The zoom-in figure shows the mechanism that drives the hydrate growth. The micro defects in the hydrate layer allow water to imbibe into the gaseous phase and coat the surface of the hydrate. This thin film becomes the new nucleation cites for the hydrate formation. This cyclic process (that is, water imbibes the gaseous phase and forms new hydrate, which further allows imbibition to happen) determines that hydrate grows into the gaseous phase. This stage is the same as in Fig. 14b.

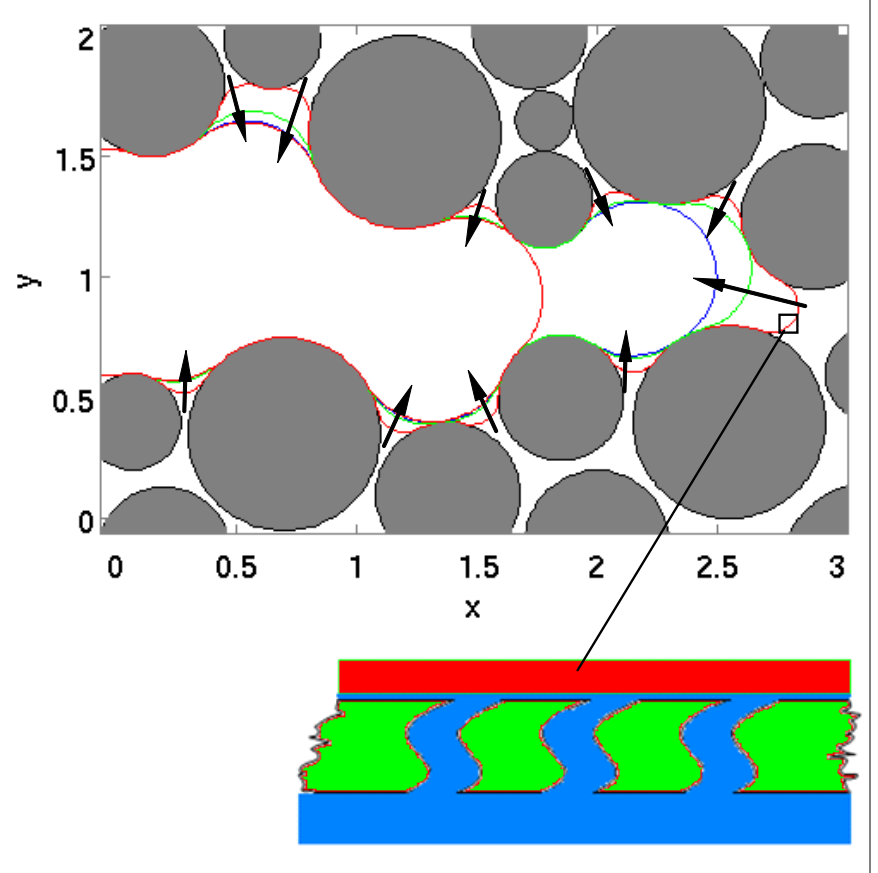


(c) Hydrate distribution (green) when the methane/hydrate interface is at the critical point. In contrast to Figure 4C, following this step, a slight decrement of curvature will result in a jump of interface to the left (Melrose imbibition jump). This is because the separate water films at the surface of the hydrate merge to form a unstable.

(d) Stage 2, the imbibition jump is an abrupt, instantaneous process. No intermediate steps, like the blue interface in Figure 4D, will be available. Thus the interface jumps as a gas/water interface to the next stable position, which is shown as the red curve. single methane/water interface, which is
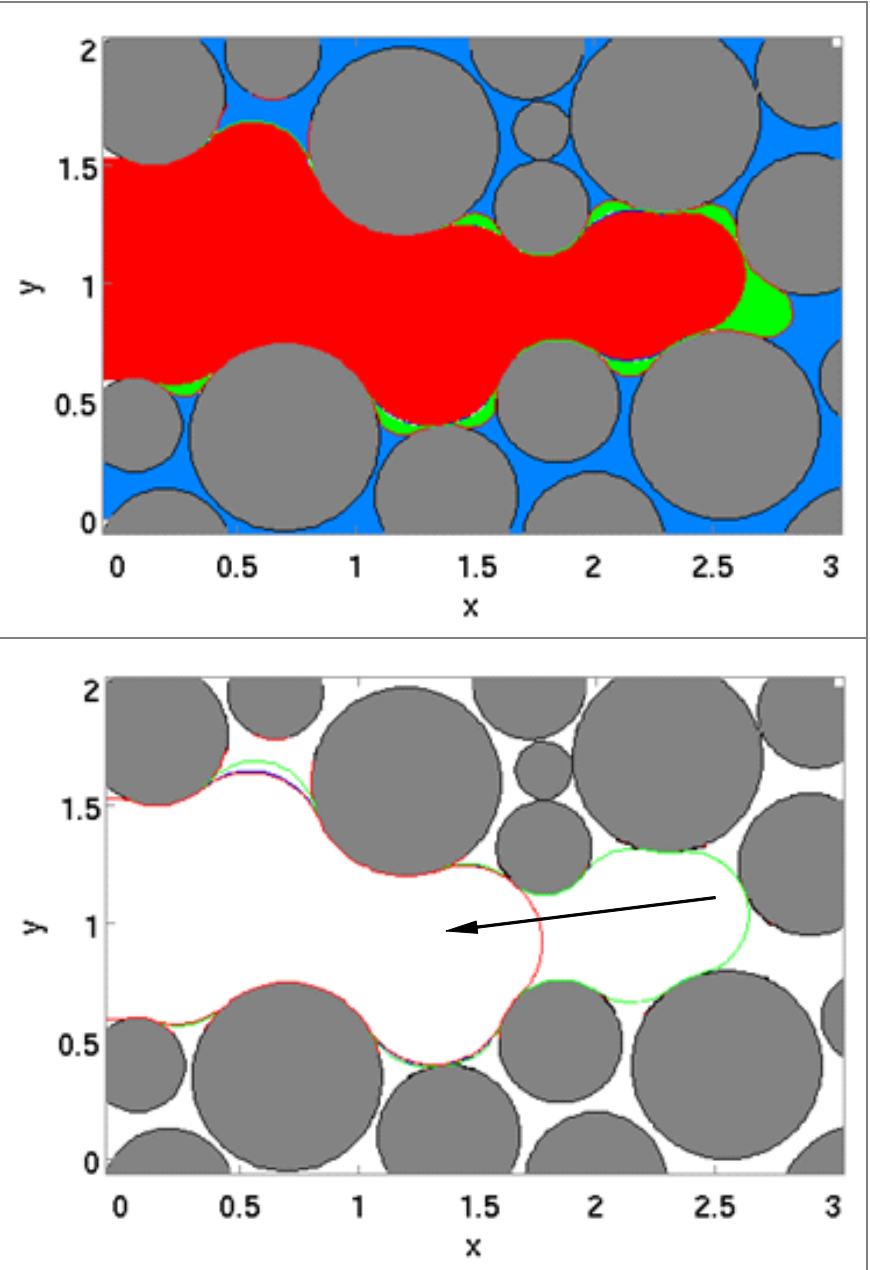

(e) Imbibition jump sucks water to fill the pore (blue). A new, stable location of the gas/water interface is reached at the end of the jump. Since this is a sudden event that gives no time for hydrate formation, water can be maintained between the two interfaces. New hydrate can form along the newly-formed gas-water interface (boundary between red and blue regions), and only grows into the gaseous phase.

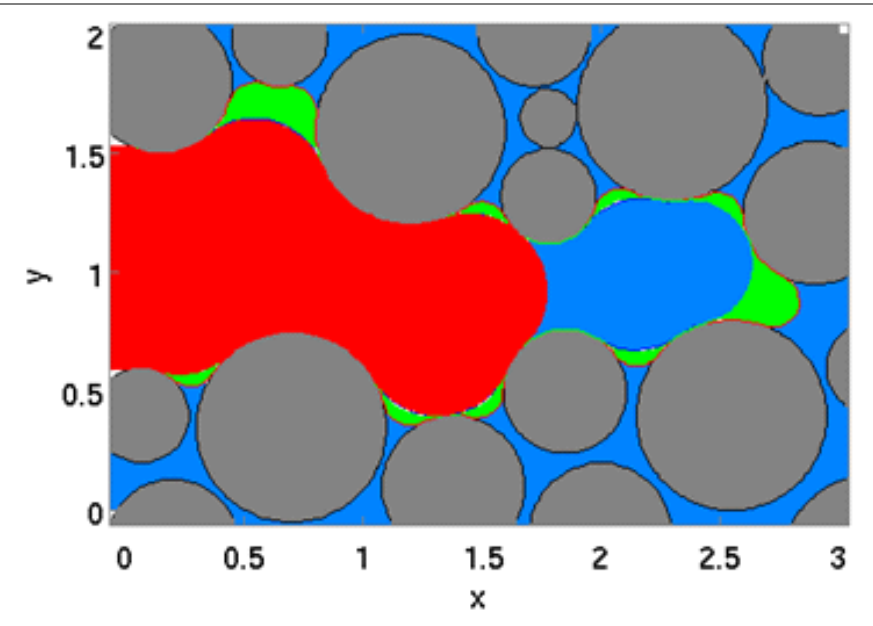


(f) Stages 1 and 2 repeat themselves cyclically. We only show here the final hydrate (green) and water (blue) distribution. The final distribution has a sandwich-like pattern: the water-filled pores are separated by hydrate shells of varying thickness. This gives the minimum hydrate saturation.

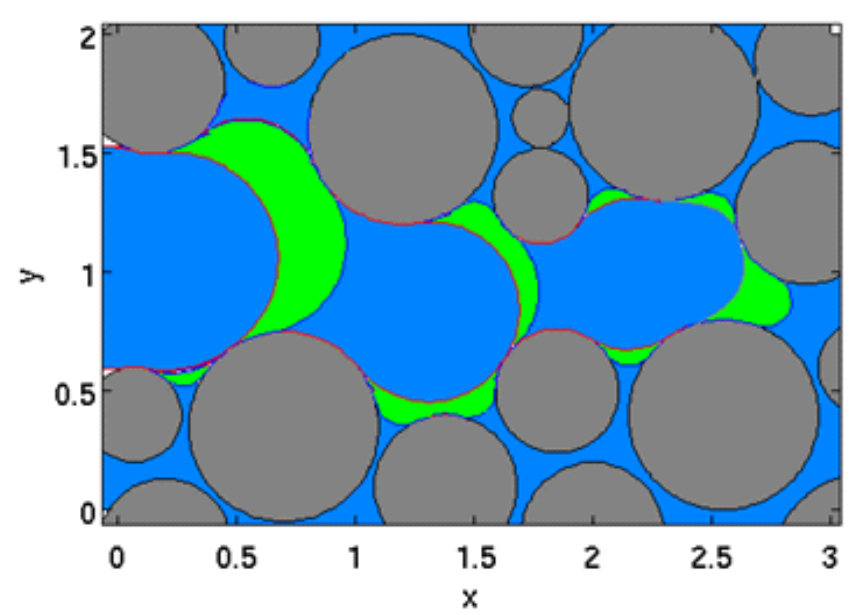

Fig. 15. Schematics of Scenario B that water supply rate is much larger than hydrate formation rate, which gives the minimum hydrate saturation. The color scheme is the same as in Fig. 14.

In Fig. 15c, Melrose jump of a gas/water interface takes place instead of the incremental movement. Consequently a large portion of the pore(s) will be filled by water. As stated by the assumption, hydrate grows only into the gaseous phase. Therefore, water invasion during the imbibition jump will not be converted into hydrate (Figs. 15d and e). Hydrate resumes growth only on the new stable locations of the gas/water interface(s), and into the gaseous phase as before. After several steps of incremental movement of the gas/hydrate interface into the gaseous phase, a Melrose imbibition jump happens again. This sequence of events leads to a sandwichlike pattern, illustrated at the imbibition endpoint in Fig. 15f: water droplets are encaged by hydrate shells in the porous medium.

It follows that this limiting case yields the minimum hydrate saturation. The original footprint of the gas phase is now occupied by water-filled pores and hydrate lenses of varying thickness, compared to the previous scenario where hydrate distribution completely follows the gas footprint. These two scenarios suggest the upper and lower limits of the hydrate saturation that could possibly happen in the porous medium: hydrate saturation is equal to the original gas saturation (upper limit, demonstrated in Fig. 14), and hydrate saturation is equal to the volume (area in 2D) swept by incremental movement (slow movement) of the interface (lower limit, demonstrated in Fig. 15). However, the requirement for the occurrence of these cases is that both methane and water are sufficient for hydrate formation (note that we do not constrain the availability of either water or methane in Scenarios A and B).

It is important to know the fraction of Melrose jump and incremental movement to estimate the hydrate saturation in Scenario B. In practice, we use network modeling to determine this value. Network modeling is a numerical tool that can be used to study drainage and imbibition (Blunt, 2001; Valvatne and Blunt, 2004; Valvatne et al., 2005; van Dijke and Sorbie, 2003). Because of the assumptions and simplifications it applies, this technique is capable of modeling fluid flow in much larger samples than LSMPQS (Peng et al., 2009). In our study, a model sediment containing 7000 monodisperse spheres is used. This model sediment is comparable to the real sediments in the hydrate reservoir, and therefore the simulation based on the model sediment is believed to represent the real fluid distribution in the porous media (Peng, 2011). By using network modeling, we obtain that Melrose jump takes 7\% saturation unit during imbibition process, and the rest of imbibition events are all incremental movements. 


\section{The Effect of Gas Trapping on Hydrate Saturation in Porous Medium}

One important behavior missing from the preceding analysis of scenarios A and B is the nonwetting phase trapping. The porous medium in Figs. 14 and 15 has large open space, and therefore does not permit trapping. In a sediments and sedimentary rocks, nonwetting phase is trapped at the imbibition endpoint, and disconnected from the bulk phase.

The trapping occurs due to two mechanisms. First, the continuous clusters of nonwetting phase are disconnected due to the imbibition happening in pores. Once the nonwetting phase loses its connection to the exit face (pores), it is trapped. Second, menisci at constraint geometries (for example, throats) merge at lower applied capillary pressure. This behavior snaps off and traps nonwetting phase in the pores. Both of the mechanisms require coalescence of separate menisci, that is, the Melrose event. A more detailed analysis can be found at (Gladkikh and Bryant, 2003).

Scenario A features a gradual invasion of hydrate, and there is no interface coalescence. Therefore, we do not consider nonwetting phase trapping in this scenario. With sufficient gas supply from other porous media, the hydrate saturation in Scenario A will be exactly the same as the initial gas saturation. On the other hand, Scenario B considers Melrose jump. Nonwetting phase will be trapped at the imbibition endpoint. When hydrate formation from the trapped gas is complete (in this case, no extra gas can be provided from the surrounding porous media), water comes in and fills the vacancy due to the hydrate formation. Much more water saturation is obtained by using this scenario.

\section{D Sedimentological Model}

Although in Figs. 11 through 13, a 2D conceptual model is used to demonstrate hydrate formation with the descending BGHSZ, the field observation suggests that hydrate profile variation is much greater in the vertical direction than the horizontal direction (Dallimore and Collett, 2005). In the Arctic hydrate reservoirs, the lateral continuity of hydrate saturation is reasonable. We therefore simplify the previous conceptual model into $1 \mathrm{D}$, which concerns only the vertical hydrate distribution. This simplification relies on the effect of grain size distribution described in the previous section. Here we extend the preceding analysis to include a highly simplified 1D model of the charging of the gas reservoirs. In effect, the model of gas charge assumes that the reservoirs are filled until the capillary barrier at the top of each reservoir is breached. It is beyond the scope of this work to consider in detail alternate schemes for gas charge, e.g. migration updip from a conductive fault. The main consequence of alternate schemes is that they need not fill the original gas reservoirs to the limits of their top seal capillary barriers. The resulting hydrate saturation profiles would still be characteristically "sandwich-like", with large saturations in the upper portion of a reservoir decreasing sharply to small saturations in the bottom portion. The difference with the model examined here would be the fraction of reservoir in which large hydrate saturation occurs.

$$
P_{C}^{e n t r y}=\frac{2 \sigma_{g w}}{R_{t h r_{-} i n s}}
$$

where $P_{C}^{\text {entry }}$ is the capillary entry pressure, $\sigma_{g w}$ is the interfacial tension between gaseous and aqueous phases. $R_{t h r_{-} i n s}$ is the throat inscribed sphere, whose value can be determined by using these approximations: 
$R_{\text {thr_ins }}= \begin{cases}\frac{1}{3} R_{\text {grain }} & \text { clean sand } \\ \frac{1}{6} R_{\text {grain }} & \text { shaly sand }\end{cases}$

where $R_{\text {grain }}$ is the median grain size of the sediment. Shale clogs the throats and therefore results in smaller throat sizes and higher capillary entry pressure. By using Eq. (31)we assume clay coats the grain uniformly in the porous medium. Equations (30) and (31) provide an approach to estimate capillary entry pressure at different layers from median grain size.

The capillary pressure between water and gas is zero at free water level (FWL), and increases almost linearly upwards. The comparison between the capillary entry pressure and capillary pressure allows the estimate of initial gas saturation in the reservoir.

\section{Grain Size Variation and Gas Charge of Mount Elbert Hydrate Reservoir}

Mount Elbert locates on the North Slope of Alaska. Cores are retrieved between $606 \mathrm{~m}$ to $760 \mathrm{~m}$, from Mount Elbert test well (Hunter et al., 2011; Rose et al., 2011). Gas hydrate was discovered from $614 \mathrm{~m}$ to $672 \mathrm{~m}$. Above $614 \mathrm{~m}$, a thick layer of clay-rich silt exists as very strong capillary seal. Modern BGHSZ is believed to be at around $900 \mathrm{~m}$, far below the depth where hydrate is observed. This observation indicates that the initial gas accumulation did not extend below this depth, and FWL (free water level) exists at $672 \mathrm{~m}$.

Figure 16 shows the median grain size and the corresponding capillary entry pressure of the sediments. Capillary pressure between water and gas is also shown for comparison, which is much greater than the capillary entry pressure. The strong contrast is due to the facts that 1) gas and water have a large density difference, and thus large capillary pressure gradient; 2) sediments have large throat openings, which lead to low entry pressure. Capillary pressure is comparable to capillary entry pressure at $650 \mathrm{~m}$. This is a potential capillary barrier to separate the reservoir into two units (Unit C and D by following Lee and Collett, 2011, and Anderson et al., 2011). 

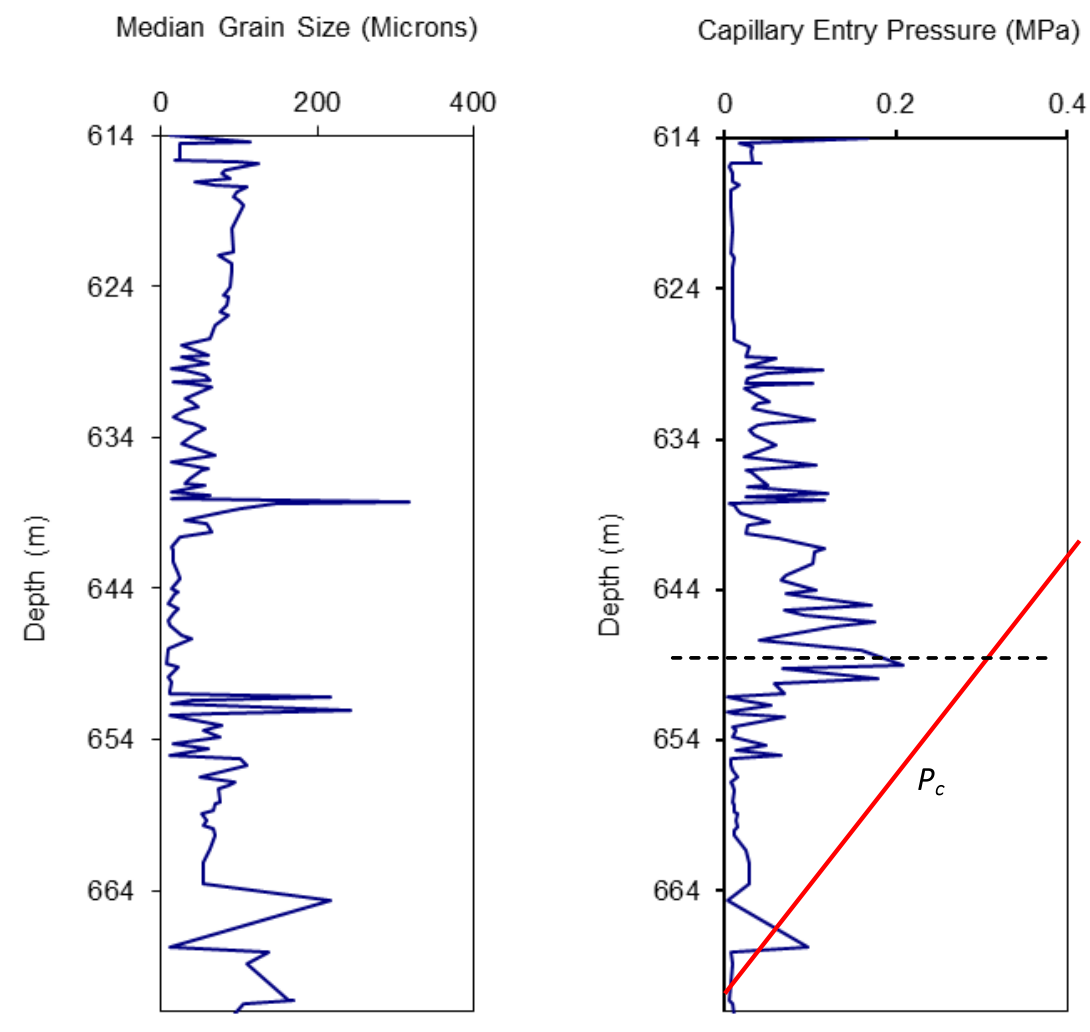

Fig. 16. The median grain size distribution and the capillary entry pressure, Mount Elbert hydrate reservoir. The capillary entry pressure is by using Eqs. (30) and (31), and the interfacial tension between water and methane to be $0.075 \mathrm{~N} / \mathrm{m}$ (this is the surface tension of water at $300 \mathrm{~K}$. This value is a weak function of salinity and pressure). The capillary pressure gradient between gas and water is $0.009 \mathrm{MPa} / \mathrm{m}$, by assuming gas density to be $50 \mathrm{~kg} / \mathrm{m}^{3}$ (at $6 \mathrm{MPa}$ and $273 \mathrm{~K}$ ) and water density to be $1000 \mathrm{~kg} / \mathrm{m}^{3}$.

At the time when BGHSZ was still above Unit D, gas generated from deeper source migrated upwards and charged these two units. When the free gas reached the capillary barrier at $650 \mathrm{~m}$, the capillary seal prohibited the further upward migration of gas. Gas therefore first accumulated in the upper region of Unit C (Fig. 17a), and expanded downwards. During the expansion, the capillary pressure at the top of Unit $\mathrm{C}$ increased. When the capillary pressure was able to exceed capillary entry pressure of the barrier between Unit C and D, gas kept migrating upwards, and accumulated from the top of Unit D (Fig. 17b). Gas slowly charged Unit D from top to bottom. This filling process continued in Unit $C$ after Unit D was saturated by gas (Fig. 17c). Similar to Unit D, gas charged from top to bottom of Unit $\mathrm{C}$. The final gas saturation profile gave a full gas column in both Unit C and D (Fig. 17d). 


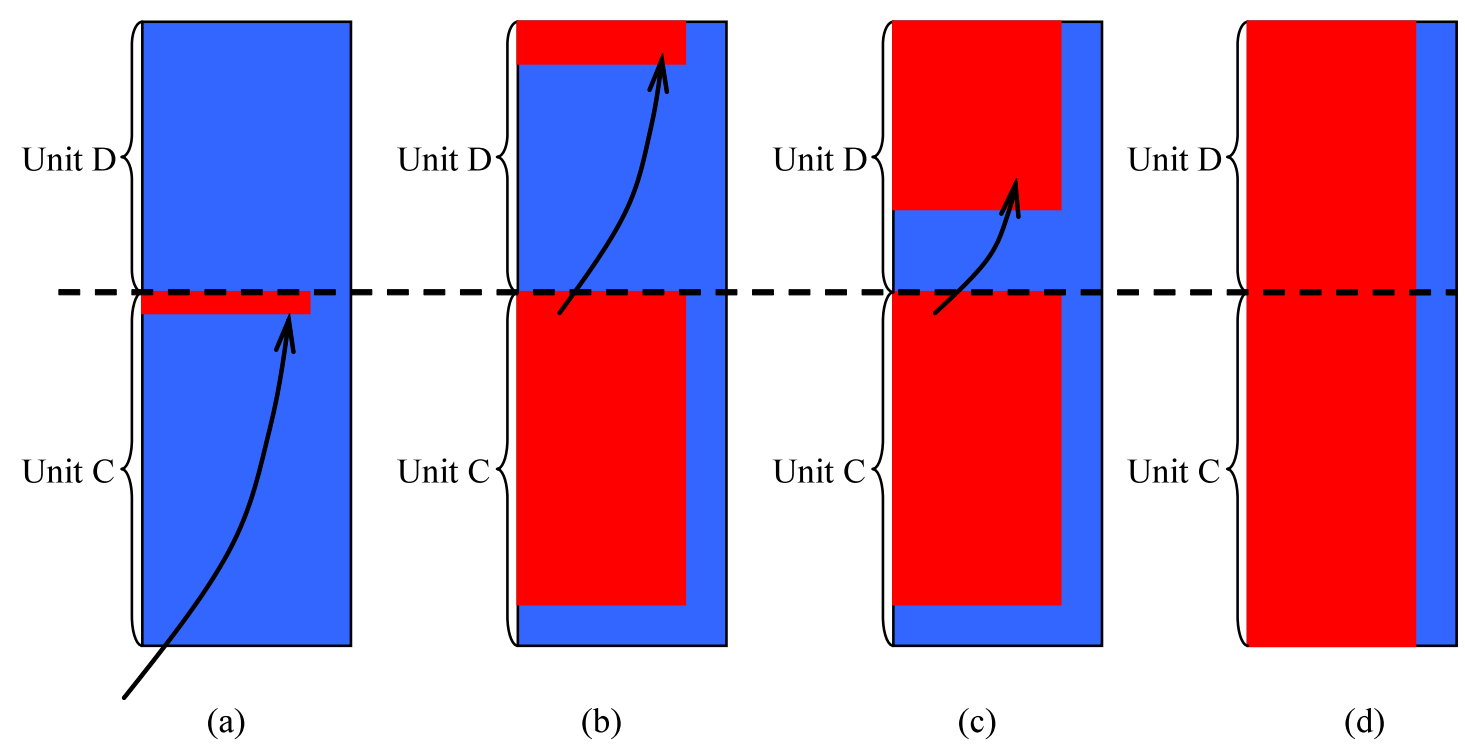

Fig. 17. Initial gas charge of Mt Elbert reservoirs subsequently converted to hydrate. (A) the upwardmovement of free gas from deeper reservoir was blocked by the capillary barrier at $650 \mathrm{~m}$. Gas accumulated beneath the capillary barrier and expanded downwards. (B) when the capillary pressure built up to exceed the entry pressure of the barrier, the upward migration restarted, and free gas began to fill the upper region of Unit D first, and expanded to the lower region (C). (D) both Unit C and D would eventually be filled up by gas.

In the current model, the initial gas saturation is uniform along the depth. This is an assumption based on the fact that capillary pressure is much higher than capillary entry pressure for most parts of the reservoir. Therefore we do not concern ourselves with the minor difference of gas saturation at different depths. We also ignore the capillary transition zone, as the length of this zone is negligible compared to the total length of the reservoir.

When BGHSZ descended and entered the gas reservoir, gas hydrate formed at BGHSZ. Depending upon the comparison between water supply rate and hydrate formation rate, different hydrate saturations were obtained (Scenarios A and B). In the following demonstration, we show only Scenario B.

Figure 18 shows the initial gas saturation, along with the capillary pressure and the sediment entry pressure. Hydrate formed at BGHSZ when it began to descend, and vacancies (due to hydrate formation) drew fluids (gas and water) from different zones: water was supplied from the capillary seal on top of the reservoir, where the permeability was comparable to the reservoir. Gas could only supply from the deepest depth where free gas was still available. At this depth, the pressure decrement due to gas loss was compensated by water invasion, from the aquifer below. The water invasion followed conventional imbibition process, where gas trapping also occurred. 


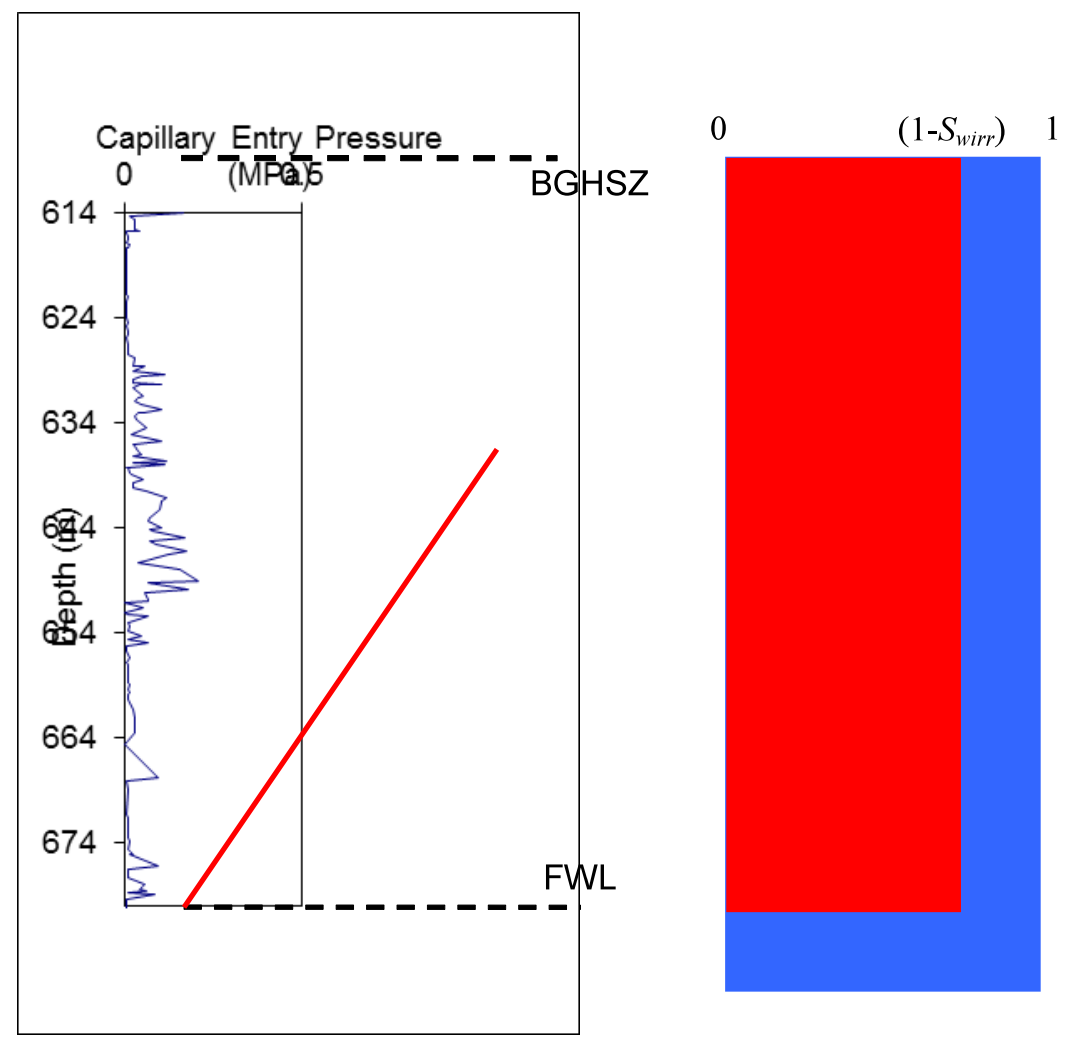

Fig. 18. The comparison of the capillary pressure and capillary entry pressure of the sediments before BGHSZ moves downwards (left), and the initial gas column (red) in the reservoir (water in blue).

The capillary pressure shifted upwards (from dashed to solid red line in Fig. 19), and reduced the difference with capillary entry pressure (Fig. 9). When the capillary pressure fell below the capillary entry pressure at $650 \mathrm{~m}$ (indicated by the arrow in Fig. 9), the gas communication between two units is terminated. We use a black line in the right panel of Fig. 9 to demonstrate this capillary barrier, which separates the gas column into upper and lower units (Unit C and D). 


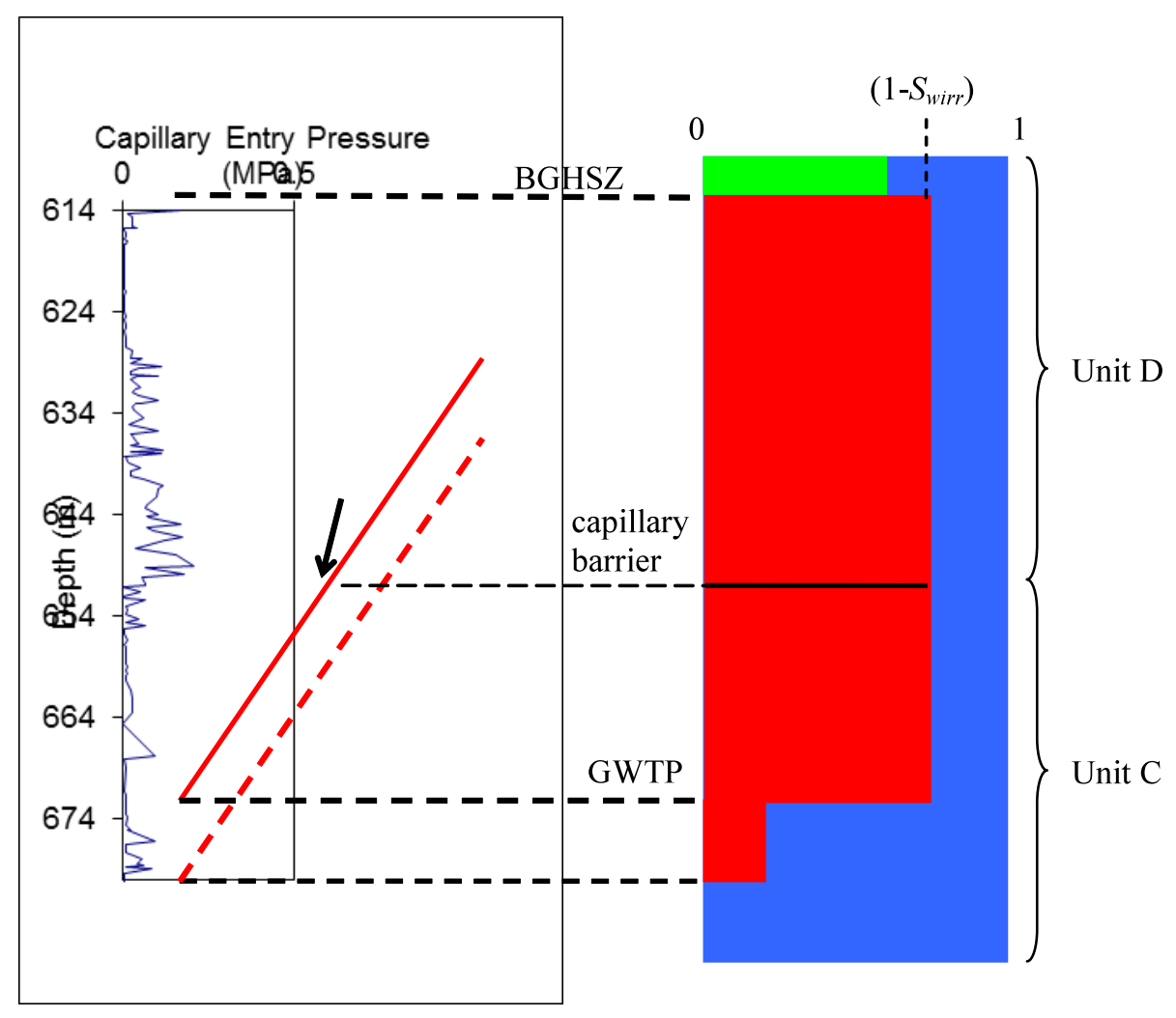

Fig. 19. The communication inside the gas column is restricted when capillary pressure decreases (from dashed to solid red line), for example due to rising gas-water contact, and becomes smaller than the capillary entry pressure. The black line on the right panel shows the possible capillary barrier that could separate the gas column into Unit C and D. GWTP stands for gas water transition point, which is the boundary between gas-saturated layers with residual-gas layers.

When BGHSZ further moved downwards, no free gas from Unit C migrated upwards due to the lack of hydraulic communication with Unit D. Instead, free gas from the bottom of Unit D (Fig. 20a) filled the vacancy created by hydrate formation and further facilitated hydrate formation. 


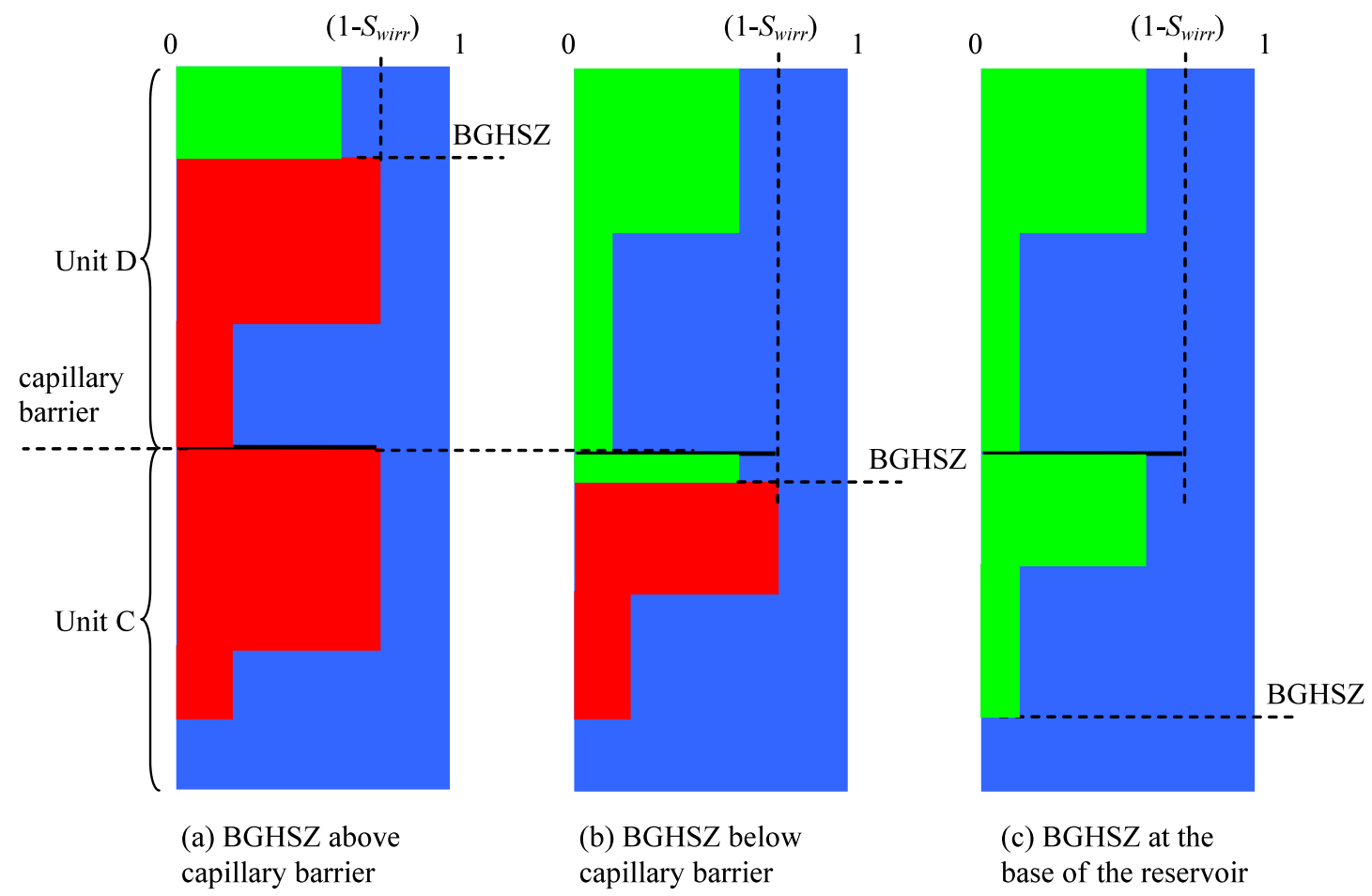

Fig. 20. Two intermediate steps when BGHSZ is above (a) and below (b) capillary barrier, and the final hydrate distribution when BGHSZ moves to the base of the reservoir (c). Almost all the free gas in (A) is supplied from the bottom of Unit D. In (b), the gas transportation is only within Unit C. The final hydrate distribution shows that Unit $\mathrm{C}$ and $\mathrm{D}$ share the similar hydrate profile.

When BGHSZ moved below the capillary barrier at $650 \mathrm{~m}$, hydrate formation and gas transportation only took place in Unit C, and followed the same process as of Unit D. Unit D and $\mathrm{C}$ had the similar hydrate distribution pattern when hydrate formation was complete (Fig. 20c).

To predict hydrate profile, we subdivide the entire reservoir into finite number of layers (for example in Mount Elbert, each unit is divided into 200 layers). When BGHSZ reaches the base of each layer, we perform the following calculation:

a. The model determines hydrate saturation in layer $i$, and calculates the equivalent amount of gas required to obtain the hydrate saturation. Here we assume that higher index means deeper layer. For Scenario A, the hydrate saturation is:

$S_{h}=1-S_{\text {wirr }}$

and for Scenario B:

$S_{h}=1-S_{n w r}\left(1-\frac{\rho_{g}}{\rho_{h}} \frac{M_{g}+N M_{w}}{M_{g}}\right)-S_{j u m p}-S_{\text {wirr }}$

where $S_{h}$ is hydrate saturation, $S_{\text {wirr }}$ is irreducible water saturation, $S_{n w r}$ is residual gas saturation. $S_{\text {jump }}$ is the volume of Melrose jump in saturation unit. $\rho_{g}$ and $\rho_{h}$ are the density of gas and hydrate, respectively.

The total mass of gas $\left(m_{g}\right)$ required to produce $S_{h}$ is computed as

$m_{g}=\rho_{h} S_{h} \phi \frac{M_{g}}{M_{g}+N M_{w}}$ 
where $\phi$ is the porosity of layer $i$.

b. The model then calculates the amount of gas needed from other layers $\left(m_{g_{-} \text {extra }}\right.$ in Eq. (35)). This is simply the total mass of gas required to form hydrate $\left(m_{g}\right)$ takes away the initial amount of gas in layer $i$.

$$
m_{g_{-} \text {extra }}=\rho_{h} S_{h} \phi \frac{M_{g}}{M_{g}+N M_{w}}-\rho_{g} S_{g} \phi
$$

where $S_{g}$ is the initial gas saturation of layer $i$.

c. Starting from the bottom layer, the model searches upwards layer by layer for available free gas that can be transported into layer $i$. For example, if layer $j(j>i)$ is already at the residual gas saturation, the model will search $j-1, j-2 \ldots(j-1, j-2>i)$, until free gas exists in that layer, say, $j-2$. If the amount of free gas in $j-2$ is not enough to compensate the amount by Eq. 35, free gas in upper layers $(j-3, j-4 \ldots$, and $j-3, j-4 \ldots<i)$ should be added until the requirement is met.

d. With the sufficient free gas coming from the deeper layer(s), layer $i$ is filled by hydrate. Then BGHSZ moves to one deeper layer $(i+1)$, and repeat steps (a) through (c). At the layers where gas saturation is already at the residual value, the model only needs to convert the residual gas into hydrate by using the following equation.

$$
S_{h}=S_{n w r} \frac{\rho_{g}}{\rho_{h}} \frac{M_{g}+N M_{w}}{M_{g}}
$$

\section{Grain Size Variation and Gas Charge of Mallik Hydrate Reservoir}

The Mallik hydrate reservoir is located on the coast of Beaufort Sea, northwest Canada (Dallimore and Collett, 2005). Most of the hydrate is found 300 to $700 \mathrm{~m}$ below the permafrost. Several test wells have been drilled since 2002, among which well 5L-38 is extensively studied. Research indicates hydrate occurrence between $890 \mathrm{~m}$ and $1110 \mathrm{~m}$. BGHSZ is located at 1100 $\mathrm{m}$. In Well 5L-38 a total of $120 \mathrm{~m}$ of hydrate column is identified, much longer than Mount Elbert.

Different from Mount Elbert, Mallik reservoir was likely to be separated into different units initially. Figure 21 shows a petrophysical description along the depth of 5L-38. At $941 \mathrm{~m}$ (the upper dashed line), the main-mode pore size (main-mode represents the dominant mode from the pore size distribution) and the gas permeability are all at their local minimum values. Since mercury porosimetry was used for the pore size determination, 'pore size' in Fig. 21 is in fact the throat size defined in this work. Therefore for $0.5 \mu \mathrm{m}$ throat size, assuming gas/water interfacial tension to be $0.075 \mathrm{~N} / \mathrm{m}$ we calculate the capillary entry pressure of this sediment to be $0.6 \mathrm{MPa}$. Using $0.009 \mathrm{MPa} / \mathrm{m}$ capillary pressure gradient, a $67 \mathrm{~m}$ of gas column, the same as the length of Unit B, must be established to overcome this capillary barrier. Therefore, an entire column of gas in Unit B will establish enough capillary pressure to barely exceed the capillary barrier at the base of Unit A. 


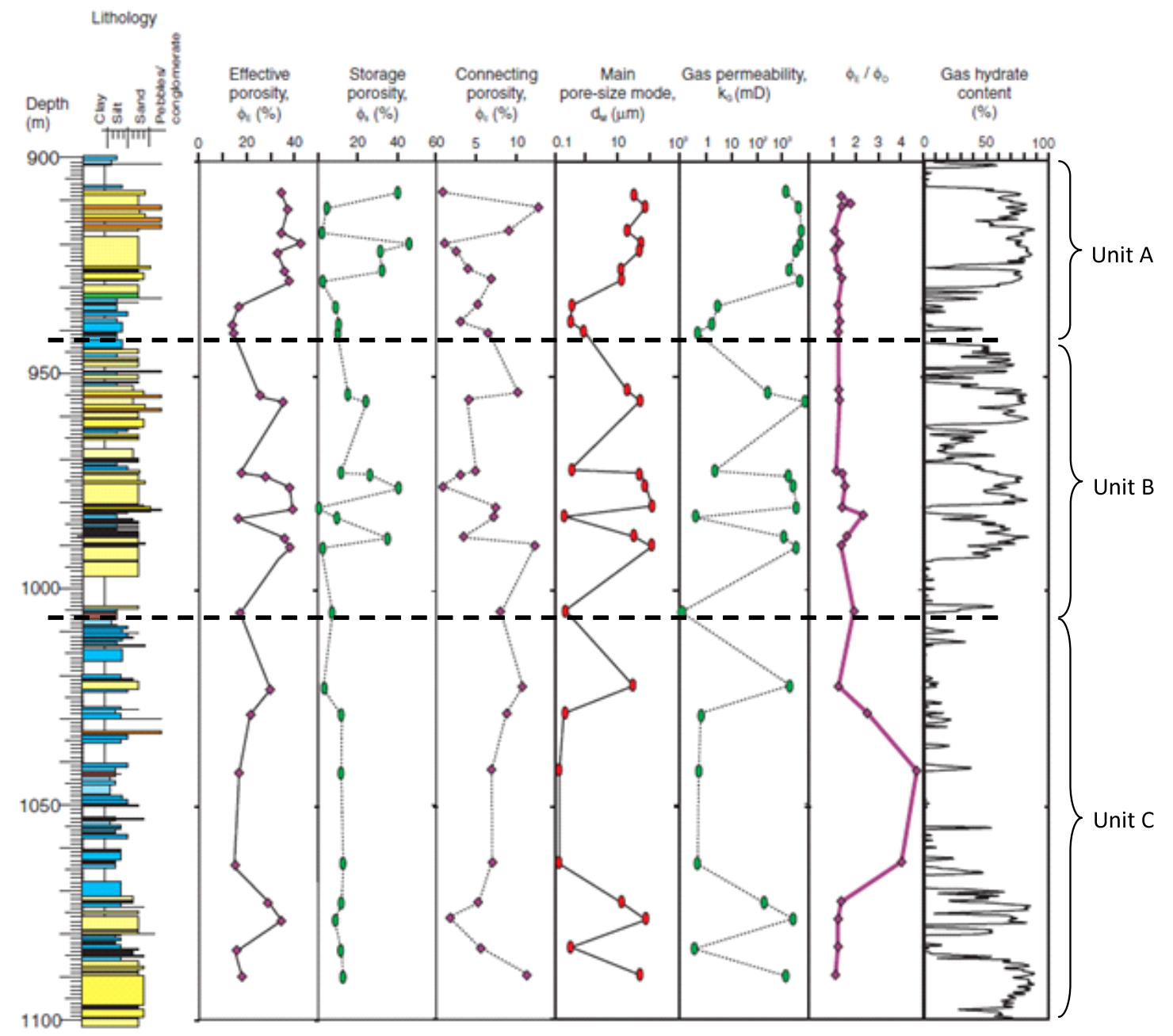

Fig. 21. The petrophysical properties from well 5L-38, Mallik hydrate reservoir. The properties are based on the lab measurements. From (Katsube et al., 2011).

At $1006 \mathrm{~m}$, near the base of Unit B, the gas permeability is at the smallest value of the entire depth, and also the main-mode pore size $(0.2 \sim 0.3 \mu \mathrm{m})$ is at its local minimum. Based on the same analysis as above, it therefore requires 1 to $1.5 \mathrm{MPa}$, or $111 \sim 167 \mathrm{~m}$ of gas column to overcome such barrier. This is close to the length of Unit C $(95 \mathrm{~m})$, considering our estimate of the 'pore size' from Fig. 21 is only approximate. We thus presume that once continuous gas column is established in Unit $\mathrm{C}$, the capillary pressure at the base of Unit B would be just sufficient to overcome the capillary entry pressure at $1006 \mathrm{~m}$.

The capillary barrier between Unit B and C separates the good lithology (Unit B) from the bad (sand-poor) lithology (Unit C). Below the barrier, except for an abnormal point $(\sim 1020 \mathrm{~m})$, the petrophysical properties (from 1006 to $1072 \mathrm{~m}$ ) all indicate very tight sediments. Along with the lithology description from (Uchida et al., 2005), we infer that water, either capillary-bound or clay-bound, dominates this region. The gas saturation only just exceeded the critical saturation in Unit $\mathrm{C}$ (this value is the minimum gas saturation that can maintain a continuous gas cluster to allow gas flow through the porous medium) to enable the gas migration pathway. Therefore, the upper part of Unit $\mathrm{C}$ allowed gas to migrate to the units above but was not drained to large gas 
saturation. This relatively small initial gas saturation explains the low hydrate saturation observed in this part of Unit C.

Another evidence of the capillary barriers is the capillary entry pressure of the sediments (Fig. 22), which is obtained by using the median grain size and Eqs. (30) and (31). In Mallik reservoir, due to the high content of shale, calculating the capillary entry pressure, especially at the shaly regions, is subject to large error. However, Fig. 22 still provides a relative comparison of the entry pressure at different depths, and therefore should only used for identifying different regions.

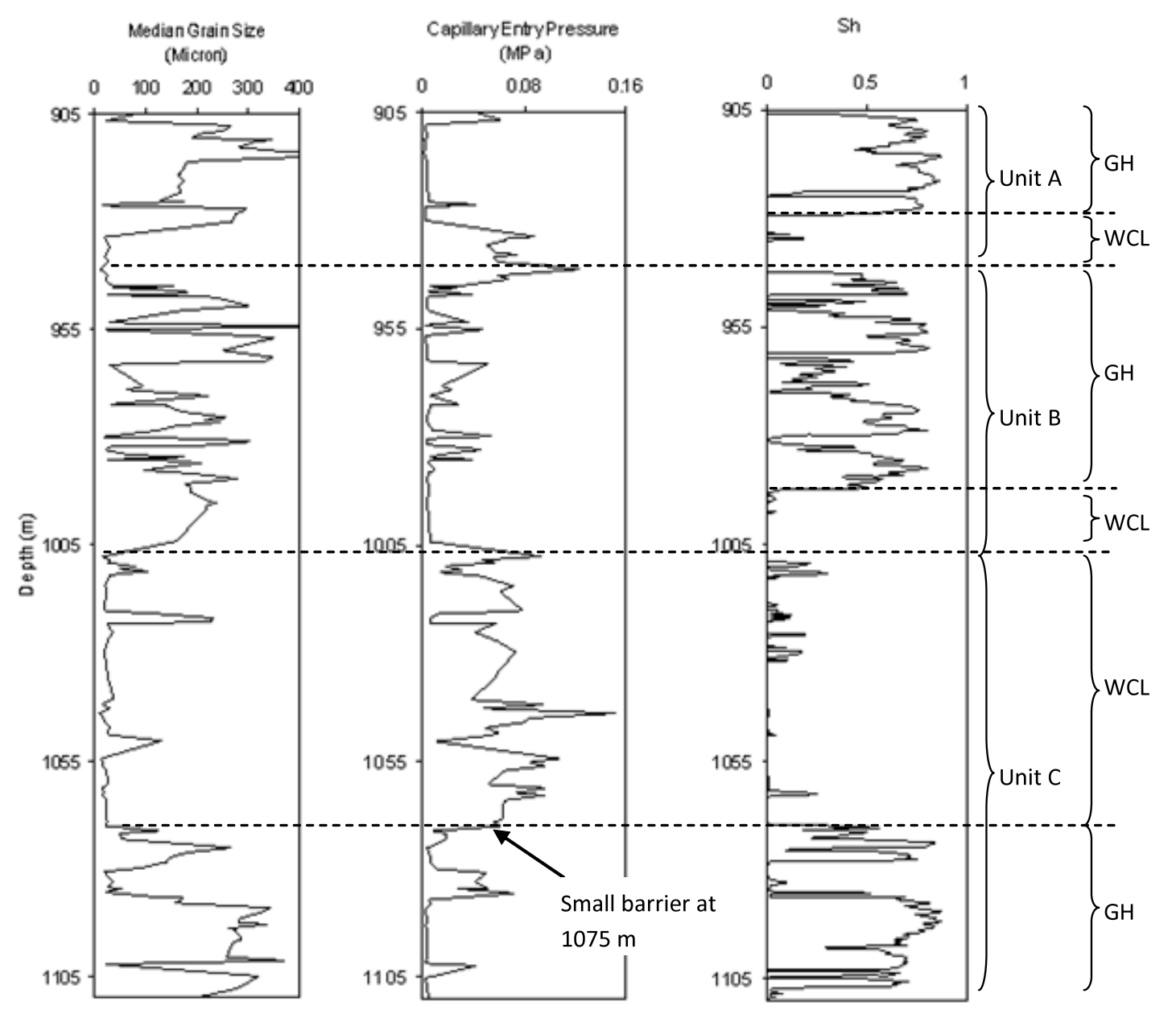

Fig. 22. The identification of different reservoir units from the capillary entry pressure, From well 5L-38, Mallik gas hydrate reservoir. The capillary entry pressure is computed by using Eqs. (30) and (31). This figure should only be used for the identification of different regions in Mallik gas hydrate reservoir, not for quantitative values of entry pressures.

To summarize the hypothesized initial distribution of gas saturation at Mallik, we assume the thermogenic gas from deeper sources migrated upwards through fractures. The migrating gas first encountered capillary barriers at $1075 \mathrm{~m}$ (Figs. 22 and 23a), thereby filling Unit C-GH (Figs 23a and 23b). The drainage curve for Unit C-GH sediments, Fig. 24, permitted buildup of large gas saturation. When the capillary pressure exceeded the entry pressure of WCL region, free gas began to migrate upwards into WCL, Fig. 13b (Recall that in Fig. 22, the capillary entry pressure 
of Unit C-WCL region and its distribution along the depth are not to scale.) The drainage curves of Unit C-WCL have different characteristics than Unit C-GH and Unit B. Because of the wider grain size distribution and higher content of clay in Unit C-WCL, these sediments have higher entry pressure and much higher irreducible water saturation (Fig. 24).

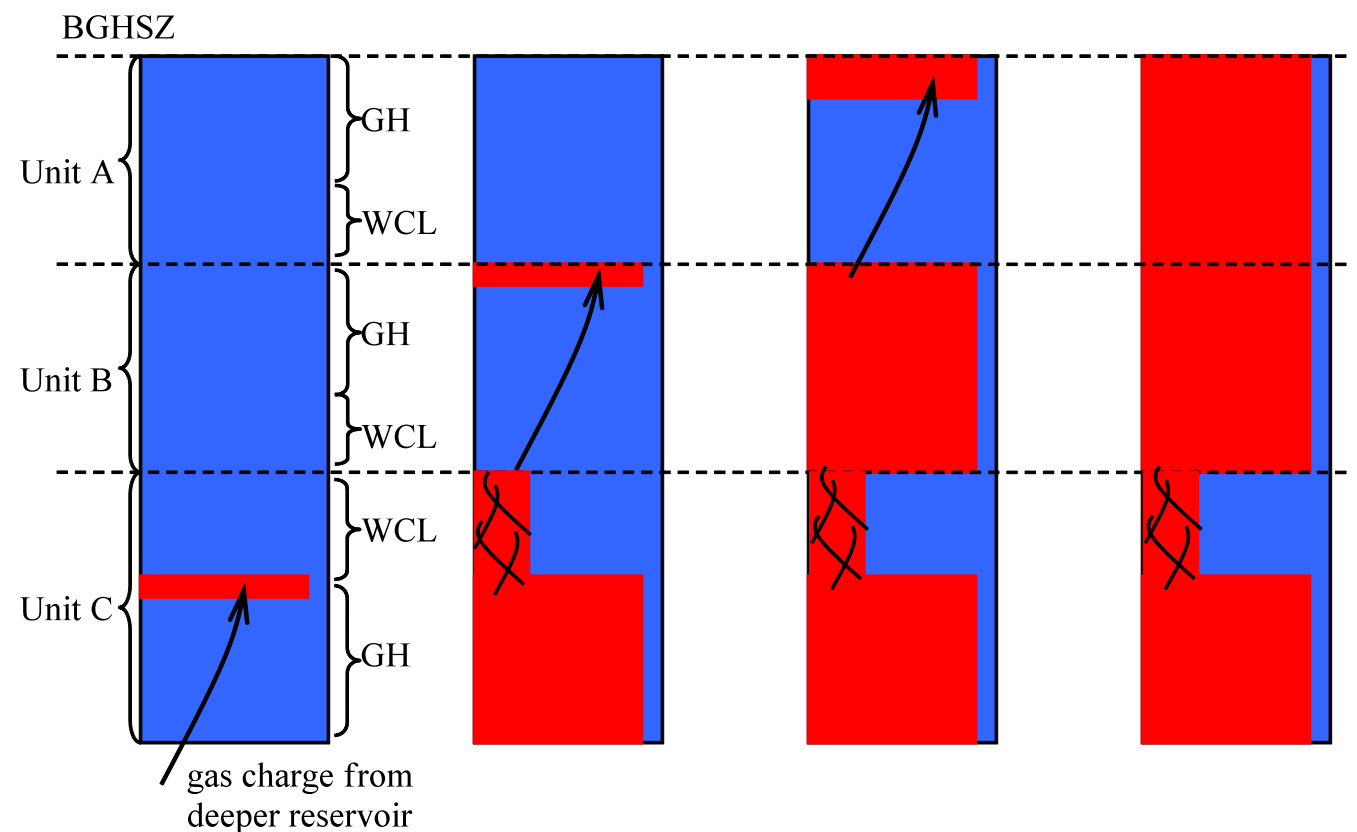

(a)

(b)

(c0

(d)

Fig. 23. Initial gas charge in the reservoir. (a) Gas invaded Unit C-GH from deeper gas source. (b) Gas filled Unit C-GH from top to bottom. When the capillary pressure exceeded the capillary entry pressure of Unit C-WCL (see WCL and GH regions in Fig. 22), gas migrated upwards through the pathways. However, the capillary pressure was not enough to allow gas to displace water in the sediments. At Unit B, gas was contained under the capillary barrier between Unit A and B, and expanded to the lower region. (c) When Unit B was almost filled up by gas, the capillary pressure exceeded the entry pressure of the barrier. Therefore, gas started to charge Unit A. Still the gas column accumulated at the top of Unit A and expanded downwards. (d) When gas charge was complete, Unit A, B and Unit C-GH were fully saturated with free gas. Unit C-WCL had small gas saturation (the minimum value to maintain the continuous cluster).

Therefore, gas column was incapable of fully draining Unit C-WCL, but was capable of maintaining the minimum saturation that would allow gas transport. When Unit $\mathrm{C}$ was filled by gas (95 m of gas column, with large saturation in Unit $\mathrm{C}-\mathrm{GH}$ and small but connected saturation in Unit C-WCL), the gas phase capillary pressure at the base of Unit B was just enough to exceed the capillary entry pressure of that sediment, and thus free gas entered Unit B (Fig. 23b). 


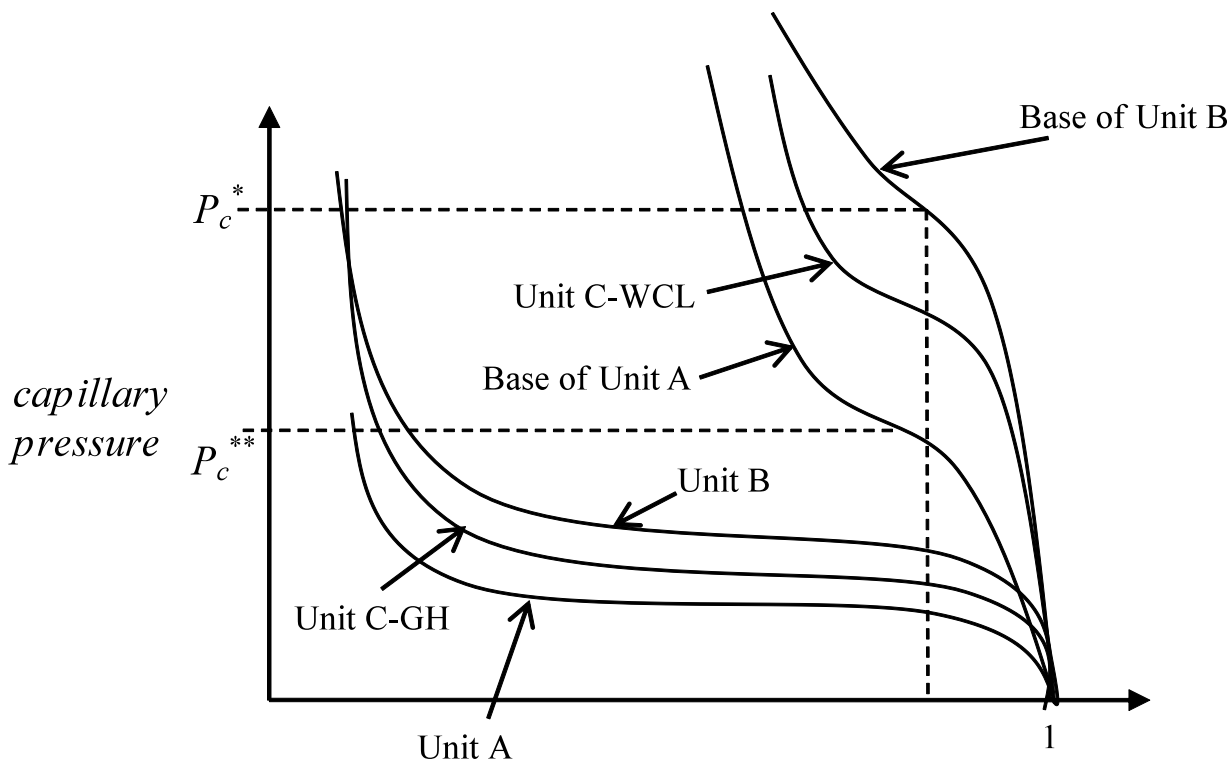

Fig. 24. Capillary entry pressure at different regions of Unit A, B and C. $P_{c}{ }^{*}$ corresponds to the capillary pressure at the base of Unit B when $95 \mathrm{~m}$ gas column occupies Unit C. At this capillary pressure, $S_{w}$ is still large in Unit C-WCL, while Units C-GH and B are drained to $S_{w, i r r} . P_{c}^{* *}$ corresponds to the capillary pressure at the base of Unit A when $67 \mathrm{~m}$ gas column occupies Unit B. At this capillary pressure, Unit A will drain to $S_{w, i r r}$.

This behavior is also demonstrated by comparing the schematic drainage curves of Unit $\mathrm{B}$ and C in Fig. 24. The drainage curves for Unit B and Unit C-GH have a low entry pressure, and also a sharp percolation. These features arise because the sediments in these regions have larger grain size and relatively narrower grain size distribution than those in WCL region, whose drainage curves in Fig. 24 have higher entry pressure and do not exhibit sharp percolation. Unit C-WCL also has larger irreducible water saturation at drainage endpoint. The capillary pressure at the base of Unit B ( $P_{c}{ }^{*}$, corresponding to $95 \mathrm{~m}$ of gas column in Unit $\mathrm{C}$ ) was just sufficient to break the capillary barrier at that depth. Once free gas entered Unit B, it displaced water to the irreducible value in accordance with the drainage curve in these coarser-grain sediments (Fig. 24).

The gas column continued to build up, until close to the base of Unit B. Because the capillary pressure at the base of Unit B was only just enough to allow gas to enter Unit B, the capillary pressure at the top of Unit B corresponds only to the thickness of the gas accumulation in this unit. As discussed above, in this situation capillary pressure at the top of Unit B was just enough to exceed the capillary entry pressure at the base of Unit A. In Fig. $24, P_{c}{ }^{* *}$ is the capillary pressure at the top of Unit B when the entire Unit B is filled by gas. When $P_{c}{ }^{* *}$ overcomes the capillary entry pressure of the barrier, gas could easily fill Unit A because of its low percolation threshold. It then allowed gas to charge Unit A (Fig. 23c). No grain size measurements are available above Unit A, so we presume the capillary barrier at the top of Unit A is enough to hold the gas column in Unit A, which is about $35 \mathrm{~m}$. At each layer except for Unit C-WCL, the initial gas saturation is set to be the constant $\left(1-S_{\text {wirr }}\right)$, because the capillary pressure between water and gas required to enter the base of each unit is more than enough to drain water to the irreducible saturation in the sands below each capillary barrier (Fig. 24).

We assume Unit A and B are not connected and thus apply the hydrate formation model to them separately. More precisely, it is assumed that the gas communication between Unit A and B 
is barely available before hydrate formation, that is, the capillary pressure at the barrier between the two units only barely exceeds the capillary entry pressure of the barrier. When hydrate first formed at the top of Unit A-GH, the upward movement of gas would reduce the gas column height in Unit B. Thus the capillary pressure at the base of Unit A immediately dropped below the entry pressure, and terminated the gas communication between Units A and B. The same phenomenon occurred within Unit $\mathrm{C}$ and $\mathrm{D}$ in Mount Elbert discussed in the previous section.

As BGHSZ descends, hydrate starts to form at BGHSZ. The scenario follows the discussion of Mount Elbert: hydrate formation generates vacant spaces, and the invading gas and water to fill the vacant spaces also become the source for further hydrate generation. Two scenarios are considered, depending on the competition between water supply rate and hydrate formation rate, which yield upper and lower bounds of hydrate saturations. In the schematic of Fig. 25, we illustrate scenario B, that is, water supply rate is much greater than hydrate formation rate, so that water invasion by Melrose jump fills part of the void space. Free gas from the bottom of Unit A migrates to fill the void space, and allows for the water invasion happening below GWTP. During this process, the state of Unit B does not change. The capillary barrier at the base of Unit A prohibits the gas communication between the two units.

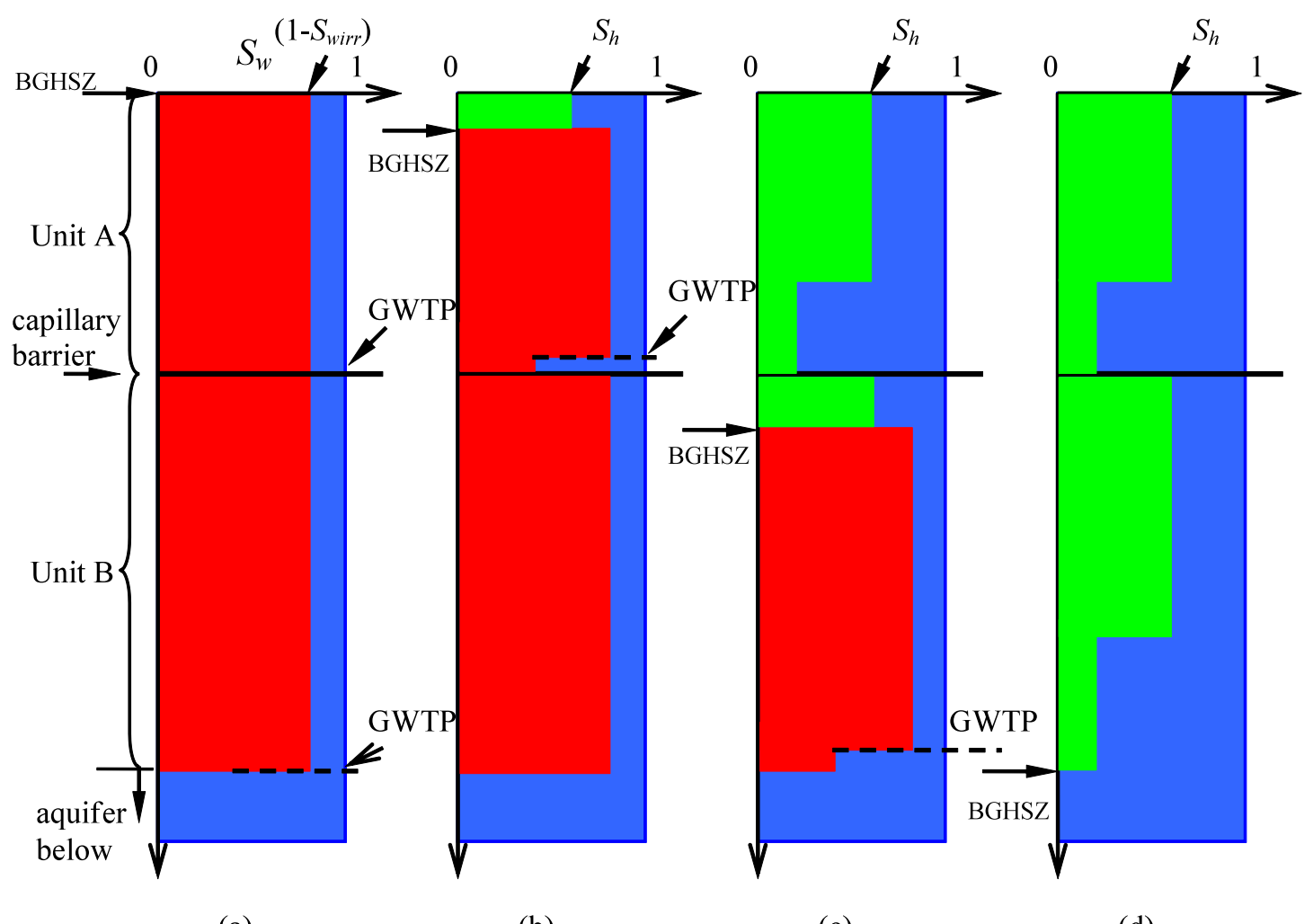

(a)

(b)

(c)

(d)

Fig. 25. A schematic of hydrate formation in Unit A and B. (a), (b), (c), and (d) show the distribution of hydrate, water and gas as BGHSZ moves downwards. It follows the similar procedure as Mount Elbert case shown in Fig. 20.

Gas in Unit A is converted into hydrate from the top down as BGHSZ moves downwards. Due to the gas redistribution within Unit A during the hydrate formation, the final hydrate distribution contains GH and WCL regions, similar to the case of Mount Elbert. GH region has the maximum hydrate saturation, while WCL region only has the hydrate saturation that is 
converted from the residual gas. Figure 25c is an intermediate step after BGHSZ moves below the capillary barrier. The same hydrate formation process repeats in Unit B, and gives a final hydrate distribution as shown in Fig. 15d.

\section{Predicting Hydrate Distribution with Pore-Scale Imbibition Model}

We predict hydrate distribution in Mount Elbert and Mallik sites by applying the imbibition models described above as the BGHSZ descends through the initial gas saturation profile, while accounting for the possibility of the gas column being disconnected vertically during conversion. Two limiting cases are evaluated, which yield the maximum (Scenario A) and minimum (Scenario B) hydrate saturation according to whether the rate of water supply is slow or fast relative to the rate of hydrate growth, respectively. The gas reservoirs are assumed not to be connected to the deeper source during the descent of BGHSZ. The model predictions for both limiting cases are compared with the hydrate saturation profiles inferred from logs.

\section{Predicted Hydrate Distribution in Mount Elbert Hydrate Reservoir}

Several important parameters must be specified in order to apply the models. $S_{\text {wirr }}$ and $S_{n w r}$ are set to be $15 \%$ and $32 \%$, respectively. These are the values from network modeling of a model sediment that accounts for periodic boundary condition, which is believed to represent the fluid distribution in the field (Peng, 2011). $S_{n w r}$ is only relevant when Melrose jump occurs (Scenario B). The saturation change associated with Melrose jumps, $S_{j u m p}$, is $7 \%$, from Section 2.1 . The density of methane $\left(\rho_{g}\right)$ is computed by using the EOS proposed by Soave, Redlich and Kwong (Sandler, 2006). Hydrate density is $910 \mathrm{~kg} / \mathrm{m}^{3}$, with the hydration number being 5.75 (Sloan, 2003). The water density is assumed to be $1000 \mathrm{~kg} / \mathrm{m}^{3}$. The porosity of the sediment varies with lithologies, and can be found in (Winters et al., 2011).

Several approaches are employed to obtain a reasonable field estimate of the hydrate saturation in the reservoir condition. These approaches include interpretations from density, resistivity, and acoustic logs (Lee and Collett, 2011), and also from core-derived chloride concentrations (Torres et al., 2011). These data are cross-verified, and thus are considered to reflect the real hydrate distribution in Unit C and D.

Figure 26 shows a comparison of the model-predicted hydrate distribution with the field data. The left panel is Scenario A where water supply rate is much smaller than hydrate formation rate. Based on the previous analysis, hydrate therefore can fill up the volume of all the original gas, and the hydrate saturation in the GH region becomes the same as the initial gas saturation in the sediment, which is $85 \%\left(1-S_{\text {wirr }}\right)$. This overestimates the saturation value from the field observation. 


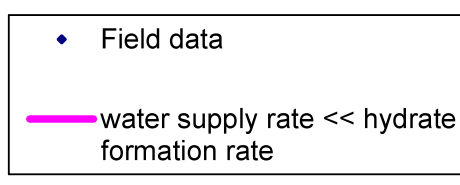

hydrate saturation

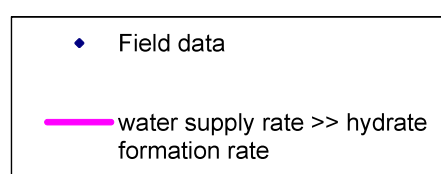

hydrate saturation

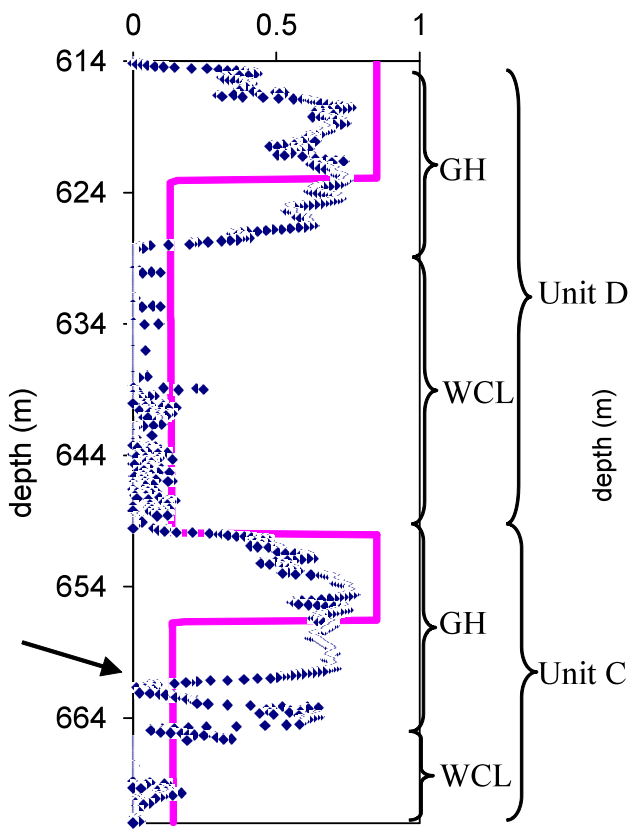

Scenario A

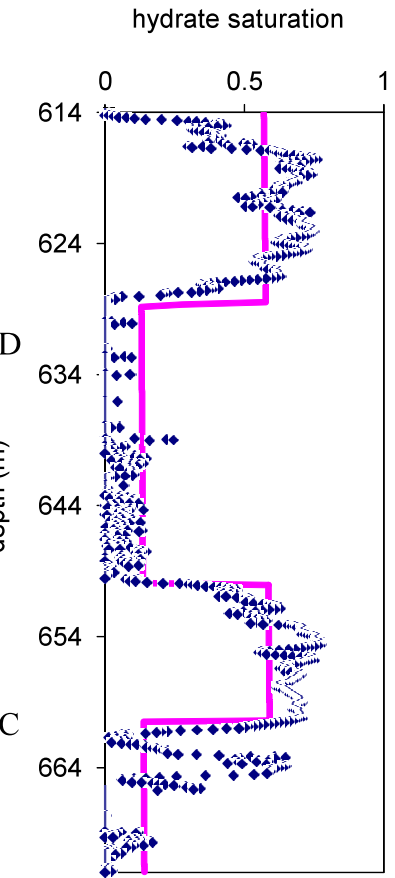

Scenario B

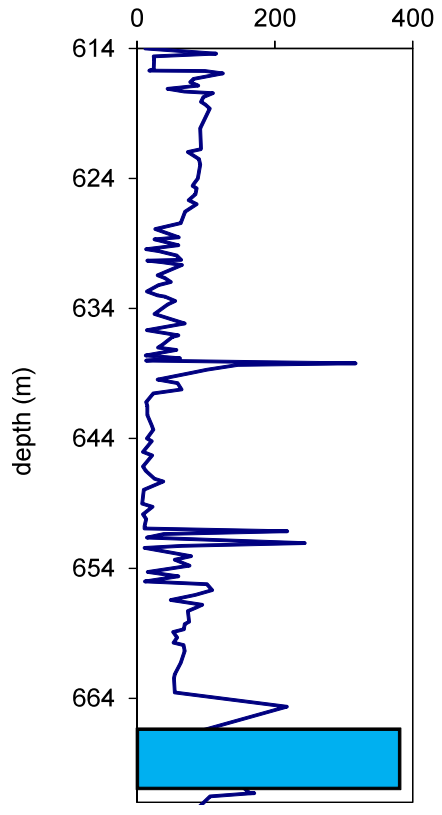

Fig. 26. A comparison of model prediction and field data of hydrate distribution, Mount Elbert hydrate reservoir (left and middle panels for slow and fast water supply, respectively) based on the pore-scale imbibition model, and the median grain size distribution along the depth (right panel).

Hydrate formation in GH region of each unit drains the free gas from WCL region, and leaves behind only the residual gas. Under the in-situ temperature and pressure, $32 \% S_{n w r}$ yields about $10 \% S_{h}$, the remaining $20 \%$ void space will be filled by water. Because Unit C and D are assumed to have no communication (Fig. 19), the same pattern of hydrate distribution is observed in both units, with higher saturation region $(\mathrm{GH})$ on top and the lower saturation region (WCL) at the bottom.

When the water supply rate is greater than the hydrate formation rate (scenario B, middle panel of Fig. 26), another imbibition event (Melrose jump) occurs. In this scenario, the void space created owing to hydrate formation will be filled by both hydrate (because of incremental movement) and water (because of Melrose jump), which gives lower hydrate saturation than Scenario A. Furthermore, gas trapping occurs at imbibition endpoint. Melrose jump disconnects gas bubbles from the bulk phase. Such behavior further increase water saturation, and gives the lower bound of hydrate saturation in the porous medium.

The hydrate saturation predicted from Scenario B is about 58\%, a much smaller value than Scenario A. With the same amount of initial gas in place, the lengths of GH region for two scenarios are different. The length in Scenario B is much longer than Scenario A, a reflection of the different maximum hydrate saturations.

The field data (blue squares in Figure 16) have the similar pattern, where GH region lays on top of WCL region. The highest hydrate saturation is around $80 \%$, lower than $85 \%$ (scenario A) 
but much higher than 58\% (scenario B). However, sediments with this saturation do not prevail along the entire $\mathrm{GH}$ region. In Unit D-GH and C-GH the average hydrate saturation is about $57 \%$ and $60 \%$, respectively. Thus Scenario B not only predicts similar hydrate saturation with the field data, but also the length of GH region. The length of GH region from prediction shows 14 $\mathrm{m}$ of Unit D-GH and $10 \mathrm{~m}$ of Unit C-GH. The corresponding values from the field data are $14 \mathrm{~m}$ of Unit D-GH and $16 \mathrm{~m}$ of Unit C-GH. We obtain a satisfactory agreement in Unit D-GH for both average hydrate saturation and unit length; however, we significantly underestimate the length of Unit C-GH.

The comparison between Scenario A and field data does not yield good agreement (Fig. 26a). The estimated hydrate saturation is much higher than, and the estimated length of GH region is much smaller than, the field data. Such comparison suggests that hydrate formation does not simply fill up gas-filled pores, but involves more complex processes (Melrose jump, Scenario B). The final hydrate saturation is a result of both of the incremental movement (hydrate filling) and Melrose jump (water filling). The good comparison between Scenario B and the field data (Fig. 26b) confirms this conclusion.

A hydrate saturation discontinuity exists at about $661 \mathrm{~m}$ (indicated by the black arrow in Fig. 26a). Our model does not predict hydrate distribution at this layer. The model estimation from Scenario B only predicts that Unit C-GH extends to $660 \mathrm{~m}$, and below that depth hydrate saturation drops to a very low value.

It is a common argument that hydrate saturation is a strong function of lithology: good lithology (clean sands with large grains) is the ideal environment for hydrate preservation (Rose et al., 2011; Winters et al., 2011), and therefore should have large hydrate saturation. In Mount Elbert case, between $662 \mathrm{~m}$ and $672 \mathrm{~m}$ (the deepest depth where hydrate is observed) we observe a good lithology (right panel of Fig. 26). However, large hydrate saturation only extends to 665 $\mathrm{m}$. Between $665 \mathrm{~m}$ and $672 \mathrm{~m}$ (indicated by the blue box) only a small amount of hydrate is observed. Based on our model prediction, we argue that such behavior is due to the fluid redistribution during hydrate formation. As demonstrated by Figs. 18 through 20, hydrate formation draws free gas from the base of each unit. Even though initially the entire unit is charged to the same gas saturation, the gas saturation is reduced to the residual value in WCL regions. The residual gas only yields a low hydrate saturation in the porous medium. Thus even though good lithology exists in WCL region it need not host high hydrate saturations after conversion of the gas reservoir.

\section{Predicted Hydrate Distribution in Mallik Hydrate Reservoir}

We use the same EOS as in the previous section on Mt. Elbert to compute gas density. Most of the properties are the same as specified in the previous section. The only different value is the hydration number. For this hydrate reservoir, this value was measured by (Ripmeester et al., 2005), who gave an average value of 6.1 over the depth. Porosity information is obtained at individual depths from (Winters et al., 2005).

The left panel of Fig. 27 shows the comparison between simulation by Scenario A and field data. In Unit A, the model prediction agrees with the maximum hydrate saturation observed in the field (85\%). However, only several layers could reach such high saturation. Only less than 20 $\mathrm{m}$ thickness of Unit A-GH is predicted, while the field data show $29 \mathrm{~m}$. The model prediction is $1 / 3$ smaller than the field observation. In Unit A-WCL, we predict roughly $20 \%$ hydrate saturation in Unit A-WCL, which is converted from the residual gas saturation $\left(S_{n w r}=32 \%\right)$. The field data, on the other hand, shows very low hydrate saturation. Since the logging data (where 
hydrate saturation data are derived from) might not be reliable when hydrate saturation is low, we do not attempt to compare the hydrate saturation in WCL region with the field data. This is also the case in Unit B-WCL.

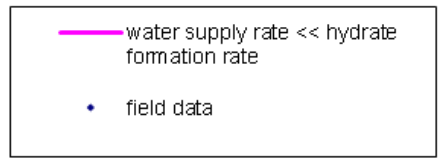

hydrate saturation

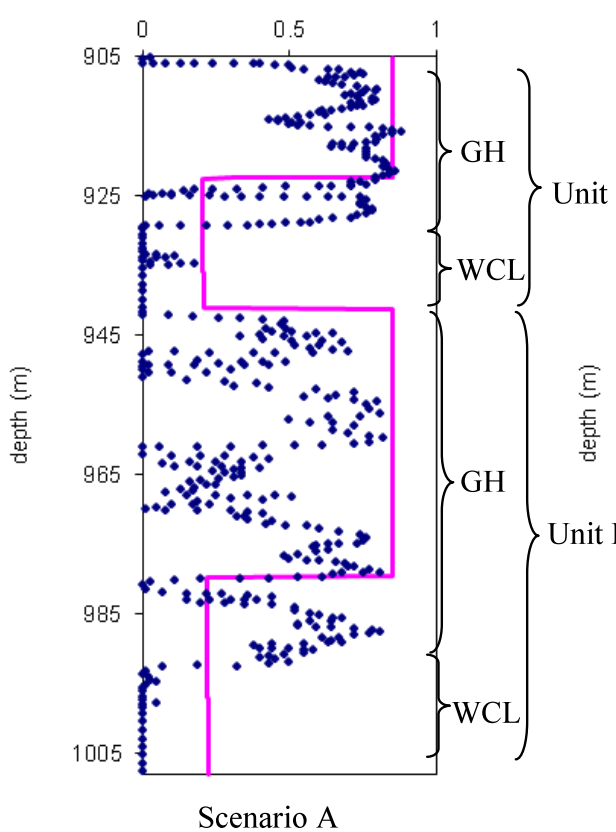

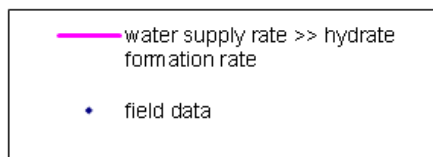

hydrate saturation

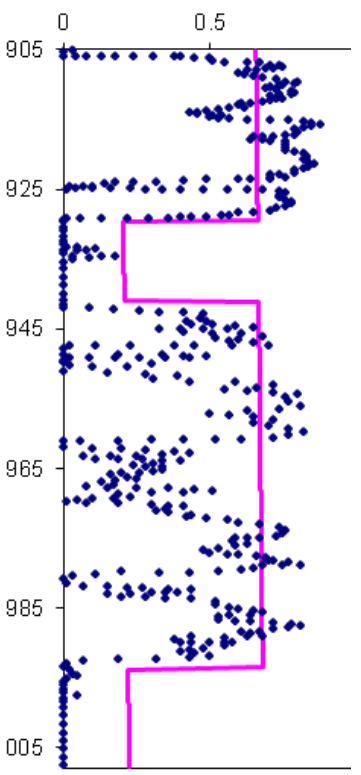

Scenario B

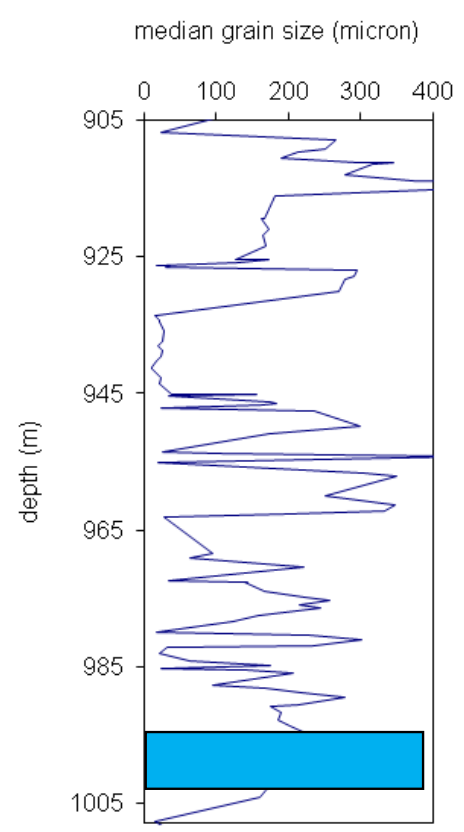

Fig. 27. A comparison of predicted and measured hydrate distribution, Mallik hydrate reservoir (left and middle panels for slow and fast water supply, respectively) based on the pore-scale imbibition model, and the median grain size distribution along the depth (right panel).

The hydrate saturation from field data has more variations in Unit B-GH. It varies between $30 \%$ to $85 \%$, which is largely dependent on the facies. In general, maximum hydrate saturation occurs in the clean sands, while the organic rich or dolomite-cemented sands have less hydrate content. Silty sands have the minimum hydrate saturation due to the higher content of clay and the secondary porosities associated with it (Winters et al., 2005). Our model does not take into consideration these features and predicts uniform hydrate saturation in Unit B-GH (left panel of Fig. 27). The prediction underestimates the thickness of GH region. By using Scenario A, we only predict $38 \mathrm{~m}$ of Unit B-GH, while the field data give $53 \mathrm{~m}$ of this region.

We study the hydrate distribution by using scenario B in the middle panel of Fig. 27. This scenario assume water supply rate to be much greater than hydrate formation rate, and therefore allows for water invasion. The maximum hydrate saturation by this scenario is lower than scenario A. It has a more satisfactory agreement with the field data. In Unit A-GH, the model prediction suggests $63 \%-65 \%$ hydrate saturation, which well matches the average saturation of the field data. The model also yields $15 \mathrm{~m}$ of the $\mathrm{GH}$ region, same with the field observation. We have a similar comparison in Unit B-GH: The model predicts almost uniform hydrate saturation $\left(S_{h}=65 \% \sim 68 \%\right)$ and similar thickness of Unit B-GH. 
Similar to Mount Elbert, we observe cases where good lithology does not correspond to high hydrate saturation. Between 1000 and $1005 \mathrm{~m}$ (indicated by the blue box) of the Mallik reservoir, clean sands with large grain size are identified. However, little hydrate is discovered in this region. Based on our analysis, we argue that this behavior is the consequence of fluid redistribution during hydrate formation. In WCL region, free gas that originally fills the porous medium is transported upwards to fill the vacancy in the shallower layers during hydrate formation. Thus only residual gas is left behind to form hydrate. This process creates hydrate depletion zone, which does not have a strong correlation with the lithology in the reservoir.

\section{Influence of Model Assumptions on Hydrate Saturation Profiles}

\section{Salinity Effect}

In the above model we assumed that solute diffusion rate is much faster than hydrate formation rate, so that the buildup of salinity in the aqueous phase in the hydrate stability zone does not inhibit hydrate formation. We now consider the opposite limiting case, in which solute does not diffuse away. Then salinity excluded from the hydrate accumulates in the remaining aqueous phase and thereby may limit the ultimate hydrate saturation.

In Mount Elbert hydrate reservoir, the background water salinity is $0.47 \mathrm{wt} \%$, which is likely to be the salinity when hydrate began to form $2.5 \mathrm{Ma}$ years ago (Torres et al., 2011). For Scenario A, we start from $S_{\text {wirr }}=15 \%$, and obtain $85 \%$ hydrate saturation. Expressed in saturation units, the total volume of water required to generate this amount of hydrate is $77.3 \%$, calculated from hydrate and water densities, which are $910 \mathrm{~kg} / \mathrm{m}^{3}$ and $1000 \mathrm{~kg} / \mathrm{m}^{3}$. Salt ions excluded from $77.3 \%$ water will remain in a volume of aqueous phase equivalent to $15 \%$ saturation when hydrate formation is complete. The salinity of the remaining aqueous phase therefore increases from its initial value of $0.43 \%$ to a final value of $2.6 \%$. For Scenario B, the same procedure can be applied, and the salinity in the aqueous phase after conversion is computed as $1.0 \%$, and tabulated in Table 1 .

Table 1. Salinity Buildup During Hydrate Formation Without Salt Ion Diffusion, Mount Elbert Hydrate Reservoir

\begin{tabular}{ccccc}
\hline & $\begin{array}{c}\text { Hydrate } \\
\text { saturation, }\end{array}$ & $\begin{array}{c}\text { Water } \\
\text { saturation, } \\
S_{w}=1-S_{h}\end{array}$ & $\begin{array}{c}\text { Initial salinity } \\
\text { (weight percentage) }\end{array}$ & $\begin{array}{c}\text { Salinity after hydrate } \\
\text { formation } \\
\text { (weight percentage) }\end{array}$ \\
\hline A & $S_{h}$ & $15 \%$ & $0.43 \%$ & $2.6 \%$ \\
$\mathrm{~B}$ & $85 \%$ & $42 \%$ & $0.43 \%$ & $1.0 \%$ \\
\hline
\end{tabular}

In Mallik hydrate reservoir, to our best knowledge there is no information of the water salinity at the time when hydrate formed. For the sake of illustration let us suppose that the salinity at the time of hydrate formation was $2.3 \%$, the same as the present-day value (Matsumoto et al., 2005). Based on the above analysis, we can also calculate the salinity after hydrate formation. The results are tabulated in Table 2. 
Table 2. Salinity Buildup During Hydrate Formation Without Salt Ion Diffusion, Mallik Hydrate Reservoir

\begin{tabular}{ccccc}
\hline & $\begin{array}{c}\text { Hydrate } \\
\text { saturation, }\end{array}$ & $\begin{array}{c}\text { Water } \\
\text { saturation, }\end{array}$ & $\begin{array}{c}\text { Initial salinity } \\
\text { (weight percentage) }\end{array}$ & $\begin{array}{c}\text { Salinity after hydrate } \\
\text { formation } \\
\text { (weight percentage) }\end{array}$ \\
\hline Scenario & $S_{h}=1-S_{h}$ & $S_{w}$ & $2.3 \%$ & $15.3 \%$ \\
$\mathrm{~B}$ & $85 \%$ & $15 \%$ & $2.3 \%$ & $5.5 \%$ \\
\hline
\end{tabular}

Figure 28 shows the maximum salinity under which hydrate can be stable in Mount Elbert and Mallik reservoirs. For Mount Elbert, hydrate is stable until salinity reaches up to $8 \%$, which is much higher than the maximum salinity we predict by assuming no salt ion diffusion (Table 1). Therefore for both scenarios in Mount Elbert case, salt accumulation does not inhibit hydrate formation.

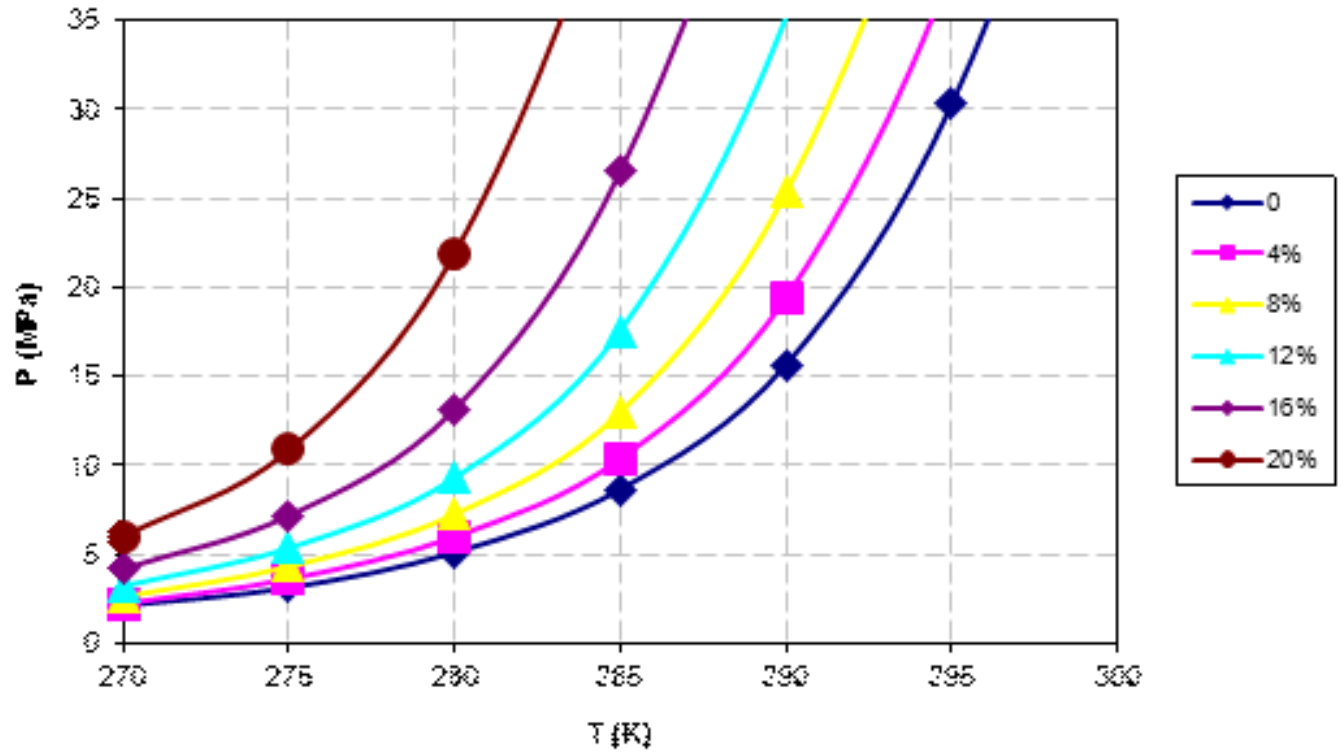

Fig. 28. GHSZ (gas hydrate stability zone) variation as a function of pressure, temperature and salinity. At each salinity, above the curve hydrate is stable, while below the curve hydrate is unstable. The pressure and temperature range of Mount Elbert and Mallik reservoirs are shown. See http://www.geochemmodel.org/models/ch4-sea/ (Sun and Duan, 2007).

In Mallik reservoir, temperature ranges from 280 to $285 \mathrm{~K}$, and pressure ranges from 8 to 10 $\mathrm{MPa}$. For Scenario A, the entire reservoir is in a region where hydrate is unstable: the salinity increases to $15.3 \%$ in Table 2 , and the curve of $16 \%$ salinity lies well above the box showing Mallik conditions. This observation suggests that for Scenario A, 85\% hydrate saturation cannot be obtained without salt ion diffusion. Scenario B, on the other hand, only has $5.5 \%$ salinity. The entire reservoir is therefore in the region where hydrate is stable. Salt ion accumulation does not inhibit hydrate formation in Scenario B.

The comparison suggests that in Scenario A the hydrate saturation should be smaller than $85 \%$. We thus would gain a similar profile with that of Scenario B (lower hydrate saturation, but longer length of $\mathrm{GH}$ region), and better comparison with the field observation. 


\section{The Direction of Hydrate Growth in the Porous Medium}

It is an assumption in our model that hydrate grows into the gaseous phase once the first nucleation occurs at the gas/water interface. Here we discuss another possibility. That is, hydrate grows into the aqueous phase instead of the gaseous phase. Based on the density of the phases, an irreducible water saturation of $15 \%$ will be converted into only $16.5 \%$ hydrate, leaving the rest of the pore space to be filled by gas $(83.5 \%)$. This result does not match the field observation from two aspects. First, the hydrate saturation in the GH region, for both reservoirs, is more than $50 \%$. Thus the prediction $(16.5 \%)$ is much lower than the field observation. Second, from the field observation only hydrate and water coexist, and no free gas is present. These two observations invalidate the possibility that hydrate grows into the aqueous phase.

\section{Summary of Influence of Pore-Scale Imbibition on Hydrate Saturation Profiles}

The imbibition model is a novel method for predicting the saturation profile of a hydrate accumulation resulting from conversion of a gas reservoir in the Arctic. The model relies on the basic physics of pore-scale processes occurring during hydrate formation. It does not account for larger-scale considerations such as the source of water supplied to the hydrate stability zone, nor the factors that control the rate of supply. Instead the model invokes the two limiting cases regarding supply rate. The results should bound what is observed in nature.

The pore scale model is inspired by the conventional imbibition process: the reduction in capillary pressure induced by hydrate formation allows water to advance into pores as gas withdraws. In the hydrate stability zone, an unconventional feature arises: hydrate forms on gaswater interfaces. Crucially, we assume the hydrate skin on a gas-water interface is permeable, allowing water to imbibe through the skin and come into contact with the gas phase. The model assumes that hydrate grows only into the gaseous phase.

The rate of permeation of water through the hydrate skin plays a significant role in determining hydrate saturation. Two limiting cases were studied. Scenario A limits the water supply rate (relative to the rate of hydrate formation), and thus prohibits the Melrose event by which most pores are filled during convention imbibition; this prohibition also eliminates the possibility of disconnecting gas phase within clusters of pores, so gas trapping does not occur. Consequently, hydrate grows to occupy exactly the same "footprint" occupied by the initial gas saturation. The final hydrate saturation and distribution is therefore identical to the initial gas. Scenario B, on the other hand, allows water supply freely, i.e. rapid compared to rate of hydrate formation. In this case the hydrate formation process at the pore scale looks more like conventional imbibition, where both Melrose jumps and the disconnection and trapping of gas in pores take place. The model identifies incremental movement of gas-hydrate-water interfaces in pore throats and at pendular rings during imbibition as well as Melrose events, in which water invades a pore or cluster of pores in a spontaneous jump. Hydrate is assumed to continue to grow at interfaces as they move incrementally. But since hydrate grows into the gas phase, the waterfilled pores cannot be converted to hydrate. On the other hand all the trapped gas is converted into hydrate. The net effect is that Scenario B yields a smaller hydrate saturation, and the hydrate occupies only a subset of the initial "footprint" of the gas phase.

The pore scale model is based on the presumption that both gas and water are sufficient for hydrate formation. This does not preclude the other assumption in the field scale, that is, the reservoir gas is the limiting phase. In this model, we assume a conventional gas reservoir before hydrate formation occurs. Gas is disconnected from the source. Thus when BGHSZ moves downwards into the reservoir (originally above the reservoir), water invasion has to occur to 
compensate the fluid loss and pressure decrease due to hydrate formation, and changes the initial fluid distribution in the field scale. Based on this mechanism, a characteristic hydrate distribution pattern is obtained. The distribution features two distinct regions: the one with high hydrate saturation and the one with low hydrate saturation. Depending on the reservoir connectivity, the pattern is often repeated in the reservoir.

Both Mallik and Mount Elbert hydrate reservoirs have hydrate distribution profiles that fall between the limiting cases predicted by the imbibition model. In fact model predictions based on Scenario B gives reasonable agreement with the field data. This suggests that the rate of water supply was large during the descent of BGHSZ.

\section{Gas Reservoir Conversion to Hydrate: Role of Pressure-Driven Fluid Phase Transport at Bed- Scale}

Overview

The immediately preceding section describes the behavior associated with conversion of gas reservoirs to hydrate reservoirs from the pore-scale perspective. That perspective did not account explicitly for the mechanism by which gas and water were supplied to the hydrate stability zone. We now turn to examine this transport problem from the bed scale. The basic premise is simple: the same decrease in gas phase pressure that drives imbibition (by reducing the capillary pressure) can also drive viscous flow of both gas and aqueous phases to the hydrate stability zone. In the earlier sections of this report that treated the overall volumetric changes associated with hydrate formation, we saw that the fraction of fluid flow made up by the gas phase controlled the hydrate saturation in the upper portions of the reservoirs. Here we develop a model of pressure-driven transport and determine whether the calculated phase fluxes yield the hydrate saturation profile observed in Mt Elbert.

The model is based on the same conceptual model for the conversion process used in previous sections. In summary, the conversion results from the descent of the base of gas hydrate stability zone (BGHSZ) through gas accumulated in a sediment. Given the measured grain size distribution in the sediment column, the initial gas phase saturation is estimated from the profile of capillary entry-pressure with depth. The gas accumulation is assumed to be isolated so that methane transport occurs only within it. We will show that if water transport occurs only by cocurrent flow of gaseous and aqueous phases up to the hydrate stability zone (HSZ), it is not possible to create large hydrate saturations from large initial gas saturations. This is because typical relative permeability curves impose strong limitations on water flux. Thus the large hydrate saturations observed in Mt. Elbert and Mallik above the current BGHSZ suggest another form of water flow: water moves down through accumulated hydrate from above. This requires the aqueous phase to remain connected within the hydrate-bearing sediment. The ratio of aqueous phase permeability in the hydrate-bearing sediment to the aqueous phase relative permeability at residual gas saturation determines hydrate saturation profile.

We begin this section by setting the context for the bed-scale transport model. The goal is to recapitulate essential features from the preceding sections of this report, so that the model development is self-contained.

\section{Context for Pressure-Driven Transport Model}

Substantial amount of research has been conducted to provide predictive models of hydrate formation in sediments. Available models can be divided into three main categories: (1) models 
predicting hydrate formation from methane-saturated water as it enters the gas hydrate stability zone (GHSZ) or by providing methane from a biogenic source in the GHSZ (Ginsburg 1998; B. Buffett 2000; Hensen \& Wallmann 2005; Bhatnagar et al. 2006; Bhatnagar et al. 2007; Bhatnagar et al. 2008; Phirani et al. 2009), (2) models assuming hydrate formation at the interface between gas phase and aqueous phase as the methane gas phase enters the GHSZ (Xiaoli Liu \& P. B. Flemings 2007; X Liu \& P. Flemings 2006; Torres et al. 2004), and (3) model presuming that the base of GHSZ moves through pre-established gaseous and aqueous phases instead of presuming fluxes of fluid(s) into the GHSZ (Behseresht \& Bryant 2011b). One motivation for the latter model was that some hydrate accumulations in the arctic such as that in Mt. Elbert, located in the southeastern portion of the Milne Point Unit (MPU) within the Alaska North Slope (ANS), are believed to be pre-established free gas columns converted into hydrate after being placed in the GHSZ (Boswell et al. 2009; T. S. Collett 1993; T. Collett 2002).

Adapting the latter model, Behseresht and Bryant (2011) achieved satisfactory matches with the hydrate saturation distribution from field data. They used a stoichiometric fixed-pore-volume model of hydrate formation from water and methane. Through considering the vertical variation in capillary entry pressure, i.e. variation in grain size distribution, as well as volume reduction during hydrate formation, leading to continuous flow of fluid phases during hydrate formation, they could mechanistically explain/predict the currently observed hydrate saturation distribution in the Mt. Elbert well deposits. Notably, the model explains why in unit C (Fig. 29a) the major methane hydrate accumulation is at the top lower-quality sand rather than the bottom betterquality deposit, and how a nearly uniform initial gas saturation profile leads to a sandwich-like hydrate saturation profile. 


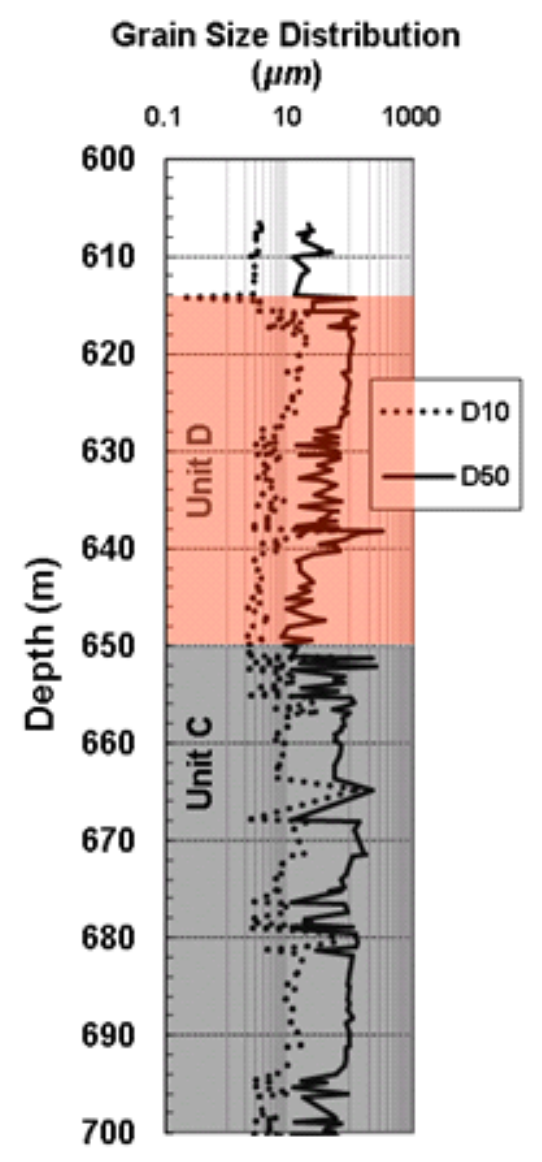

(a)

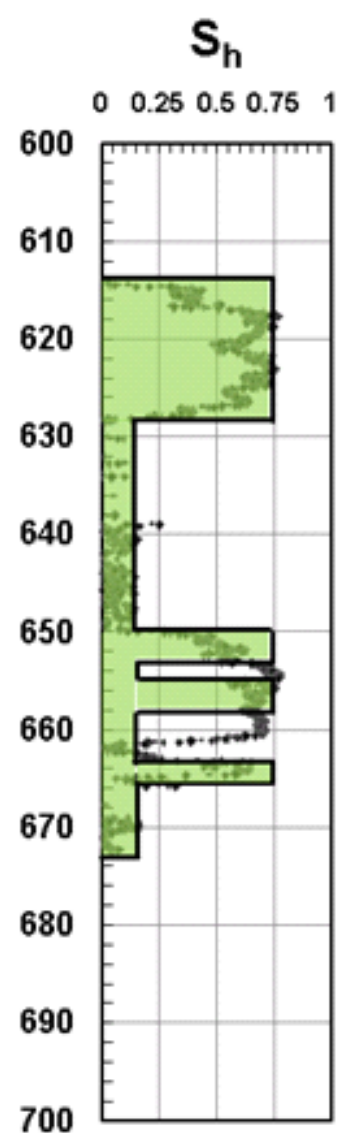

(b)

Fig. 29. (a) The $10^{\text {th }}\left(\mathrm{D}_{10}\right)$ and $50^{\text {th }}\left(\mathrm{D}_{50}\right)$ percentile of grain size extracted from core data (Rose et al. 2011) versus depth in Mt. Elbert well. The depth range shown, 600-700m, includes two sand units informally designated as sand unit D and C by Collett (T. S. Collett 1993). (b) Predicted final hydrate saturation (green area) by Behseresht and Bryant model forms a sandwich of large and small values. An $R_{v}$ value of $\approx 0.55$ was used in this case. The log derived hydrate saturations are shown as dots (Behseresht \& Bryant 2011b).

It was shown that to have a hydrate saturation distribution as currently observed in Mt. Elbert, abundant amount of fluids, up to 1.4 pore volumes for Mt. Elbert case, should have been transported into the hydrate-bearing zones. It was also shown that the gas phase volume fraction, $R_{v}$ in Eq. (37), of the total transported fluid volume, $\Delta V_{g}+\Delta V_{w}$, is directly related to the final hydrate saturation in a sediment zone, given the initial fluid phase saturations, $S_{w, i}$ and $S_{g, i}$. (Behseresht \& Bryant 2011b). That work did not propose a mechanism for the fluid transport, however.

$$
R_{v}=\frac{\Delta V_{g}}{\Delta V_{g}+\Delta V_{w}}
$$

In this section we review some key elements of the stoichiometric model applied to model hydrate formation in porous media and then discuss an important new aspect of the model: What parameters control the value of $R_{v}$ and thus the resulting hydrate saturation in the host sediments. It is assumed that the alternative to fluid movement as a result of volume reduction associated with hydrate formation, which is compaction or grain rearrangement within the sediment, can be 
neglected (Behseresht \& Bryant 2011b). We also assume that methane that moves to the GHSZ is subsequently converted to hydrate. For simplicity we also assume that $\mathrm{CH}_{4}$ is the only constituent of the gas phase and the only guest molecule in the hydrate. We assume that the aqueous phase has a small initial salinity and that any buildup in salinity caused by incorporation of $\mathrm{H}_{2} \mathrm{O}$ into hydrate is rapidly dissipated into an unlimited reservoir of brine. The gas column is assumed to be no longer connected to its source and thus the gas mass is fixed. Finally, the heat diffusion coefficient is about 500 times larger than the salinity transport coefficient, so we neglect the effect of heat transfer on limiting hydrate formation.

Figure 30 shows a box of sediment with total pore volume $V_{\text {tot }}$ containing an initial water saturation and methane saturation of $S_{w, i}$ and $S_{g, i}$, respectively. For the sake of visualization, the gas and aqueous phases are shown as if they occupied separate subvolumes. The box of sediment is assumed to be open to methane and water flow to compensate for the volume reduction associated with the hydrate formation inside the box. It is assumed that the thermodynamic condition, $P$ and $T$, is appropriate for hydrate formation inside the box and hydrate keeps forming until one of the constituents inside the box, water or methane, is entirely consumed. The final hydrate saturation, $S_{h, f}$ inside the box is governed by the proportion of gas and water entering the sediment, i.e. $R_{v}$, as shown in Eq. (38).

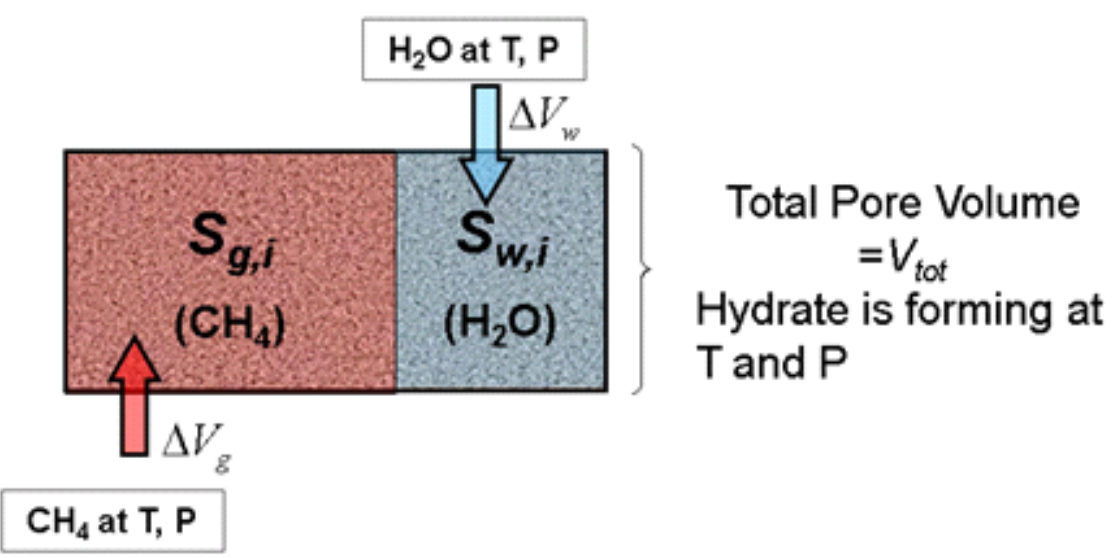

Fig. 30. Sediment box with total pore volume $V_{\text {tot }}$ filled with water and methane. The sediment is under a pressure/temperature suitable for hydrate formation. The box is open to water and methane flow from outside.

$S_{h, f}=\left\{\begin{array}{cc}\frac{\bar{V}_{h}}{R_{v}\left(\bar{V}_{h}-\bar{V}_{g}-N \bar{V}_{w}\right)+\bar{V}_{g}} S_{g, i} & ; R_{v}<R_{v, \text { stoich }} \\ 1 & ; R_{v}=R_{v, \text { stoich }} \\ \frac{\bar{V}_{h}}{\left(\bar{V}_{h}-\bar{V}_{g}\right)\left(1-R_{v}\right)+N \bar{V}_{w} R_{v}} S_{w, i} & ; R_{v}>R_{v, \text { stoich }}\end{array}\right.$

Here $\bar{V}_{h}, \bar{V}_{w}$ and $\bar{V}_{g}$ are hydrate molar volume, water molar volume and methane molar volume at pressure $P$ and temperature $T$. $R_{v, \text { stoich }}$ is defined as a value of $R_{v}$ for which $S_{h, f}=1$. It can be shown that $R_{v, \text { stoich }}$ is related to the initial gas saturation by Eq. (39).

$R_{v, \text { stoich }}=\frac{\bar{V}_{g}-\bar{V}_{h} S_{g, i}}{\overline{\bar{V}}_{g}+N \bar{V}_{w}-\bar{V}_{h}}$ 
Given an initial gas/water saturation inside the sediment box in Fig. 30, the dimensionless volume of fluids transported into the sediment, $\Delta V_{g, d}$ and $\Delta V_{w, d}$, is related to the final hydrate saturation inside the sediment by Eqs. (40) and (41). Dimensionless volumes are in units of pore volume and are calculated by dividing the absolute volumes, $\Delta V_{g}$ and $\Delta V_{w}$, by the total pore volume of the sediment, $V_{\text {tot }}$.

$$
\begin{aligned}
& \Delta V_{g, d}=R_{v}\left(\frac{N \bar{V}_{w}+\bar{V}_{g}}{\bar{V}_{h}}-1\right) S_{h, f} \\
& \Delta V_{w, d}=\left(1-R_{v}\right)\left(\frac{N \bar{V}_{w}+\bar{V}_{g}}{\bar{V}_{h}}-1\right) S_{h, f}
\end{aligned}
$$

The gas phase fraction denoted by $R_{v, 1: 1}$ represents a neutral point between imbibition (i.e. increase in $S_{w}$ from its initial value, $S_{w, i}$ ) occurring during hydrate formation for $R_{v}<R_{v, 1: 1}$ and drainage (i.e. decrease in $S_{w}$ from its initial value, $S_{w, i}$ ) occurring for $R_{v}>R_{v, 1: 1}$. They showed that the value of $R_{v, 1: 1}$ is independent of initial saturations (Eq. (42)):

$$
R_{v, 1: 1}=\frac{\bar{V}_{g}-\bar{V}_{h}}{N \bar{V}_{w}+\bar{V}_{g}-\bar{V}_{h}}
$$

In summary, this model allows the full range of behavior, from only aqueous phase entering the sediment box to fill the volume $\left(R_{v}=0\right)$ to only gas phase entering $\left(R_{v}=1\right)$. For $0<R_{v}<$ $R_{v, 1: 1}$ the aqueous phase coexists with hydrate at the final state and $S_{w, f}=1-S_{h, f}<S_{w, i}$, so that imbibition occurs during hydrate formation. For $R_{v 1: 1}<R_{v}<R_{v, \text { stoich }}$, aqueous phase and hydrate coexist at the final state, but some drainage has occurred, so that $S_{w, f}=1-S_{h, f}>S_{w, i}$. Finally, for $R_{v, \text { stoich }}<R_{v}<1$, the gas phase coexists with hydrate at the final state, with $S_{g, f}=1-S_{h, f}$. The situation commonly encountered in regions between the base of permafrost and the BGHSZ is an aqueous phase coexisting with hydrate. The hydrate phase can be present in trace amounts, $S_{h}<$ 0.10 , or in rich layers (many meters of sediment) with $0.5<S_{h}<0.8$. In the latter case, of order one pore volume of gas and aqueous phases must move into the layer, with roughly equal volumes of each phase.

\section{Flow Model for Gaseous and Aqueous Flow into the GHSZ}

The driving force for fluid phase movement is the reduction in volume occupied by the gas/aqueous/hydrate phases when hydrate forms from gas and aqueous phase components. We wish to determine gas phase and aqueous phase movements independently, and thereby determine the value of $R_{v}$. To do this it is first necessary to know the mechanisms through which gaseous and aqueous phase arrive into the zone of hydrate formation while hydrate is forming in an interval newly introduced into the GHSZ. Figure 31 illustrates a sand unit with a gas column established below a seal at depth, $h_{1}$. 


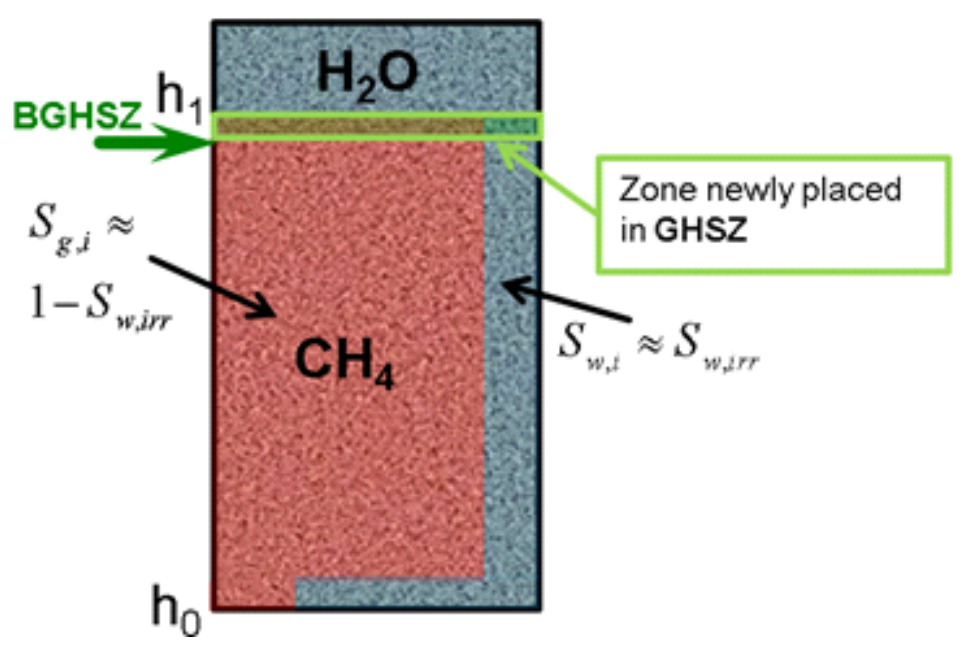

Fig. 31. A sand unit having a seal at depth, h1 is shown. A gas accumulation is established below the seal. The BGHSZ was above the gas column when the gas column was established. The BGHSZ is descending through the sediment column. The zone recently placed in the GHSZ is shown within a green fill on the sediment column.

The BGHSZ has just moved into the gas-bearing zone and hydrate can form inside the zone shown with a green box. Because of the very low ratio of aqueous to gas phase mobilities, steady co-current flow of aqueous phase and gas phase from below the BGHSZ cannot supply the amount of water required, $\Delta V_{w, d}$ (Behseresht \& Bryant 2011a). Instead, a piston-type movement of gas would arise, with the aqueous phase following much more slowly from below, as shown in Fig. 32.

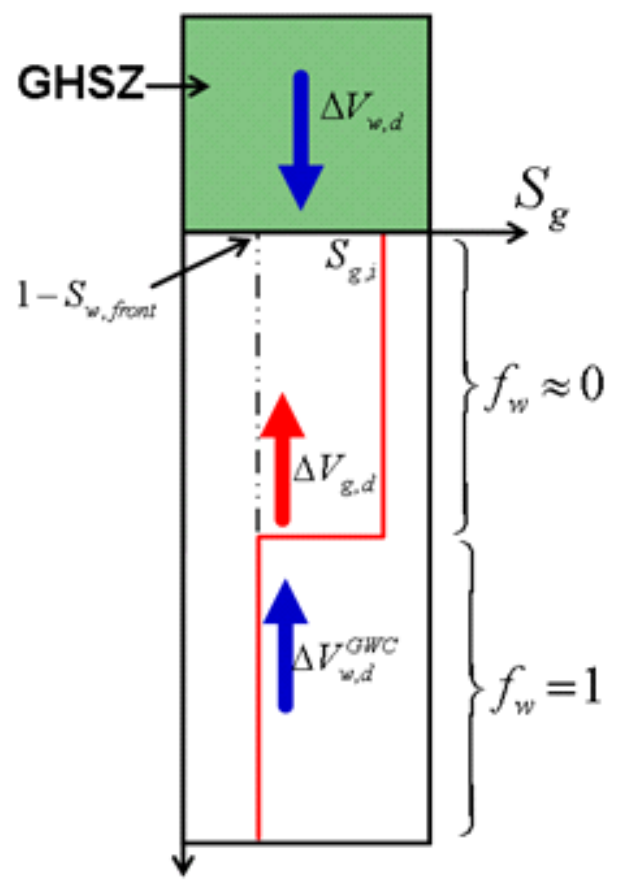

Fig. 32. Schematic for the transported volumes of aqueous and gas phase when hydrate is forming at the base of GHSZ. Fractional flow theory proves it unlikely that the required amount of aqueous phase, $\Delta V_{w, d}$, has been transported through an upward co-current flow with the gas phase. Instead we suppose it to have come through the hydrate-bearing sediment above BGHSZ. 
Because aqueous phase viscosity is much larger than that of the gas phase, the aqueous phase mobility $\lambda_{w}$ is much smaller than that of the gas phase $\lambda_{g}$ for almost any reasonable set of relative permeability curves (Behseresht \& Bryant 2011a). Consequently, the saturation just upstream of the front, $S_{w, f r o n t}$, would be almost the same for all initial water saturations: $S_{w, \text { front }} \approx 1-S_{g r}$, where $S_{g r}$ is the residual gas saturation. The corresponding fractional flow of water would be $f_{w, \text { front }} \approx 1$. Just ahead of the front, the water saturation is $S_{w}=S_{w, i}$ and $f_{w} \approx 0$. Thus only gas is arriving $\left(f_{g}=1-f_{w} \approx 1\right)$ at the BGHSZ. This piston type movement of a methane bank ahead of the water bank can provide the upper zones in which hydrate is forming with the needed gas, $\Delta V_{g, d}$. Simultaneously this piston type movement of aqueous phase would require the transport of water, $\Delta V_{w, d}^{G W C}$, to replace transported gas from below and thereby raise the gas-water contact $(G W C)$. But clearly the cocurrent flow cannot have transported water into the zone of hydrate formation.

The need for considerable amount of water, $\Delta V_{w, d}$, during hydrate formation requires a different route for water flow. We propose that water moves down through accumulated hydrate from the unfrozen water above (Fig. 32). (Such water exists, because the base of permafrost is always above the BGHSZ.) For this to happen the water phase must remain connected within the hydrate-bearing sediment. This requires the final hydrate saturation at the upper zones to be less than $1-S_{w, i r r}$ so the aqueous phase is connected at the final water saturation. Behseresht and Bryant (2011b) predict that this condition will arise, because imbibition occurs in the upper zones as the GWC rises and causes the capillary pressure to decrease as the height of the gas column decreases.

In the following, the model illustrated in Fig. 32 is formulated and applied on one of the sand units in Mt. Elbert well. Gas is assumed to have a piston type cocurrent flow. Figure 33 shows the sand unit in Fig. 31 when the BGHSZ has moved down through a portion of the original gas column. The figure shows the situation once the BGHSZ has moved from depth, $h_{1}+L_{h}$ to $h_{1}+L_{h}+\Delta L_{h}$. The gas accumulation between $h_{1}$ and $h_{1}+L_{h}$ has been fully consumed to form hydrate and the pore space is filled with hydrate and water only, i.e. $R_{v}<R_{v, \text { stoich }}$. As hydrate forms in the $\Delta L_{h}$ interval newly placed in the GHSZ, the flow of aqueous phase, $q_{w}$, and gas phase, $q_{g}$, takes place. Aqueous phase pressure is assumed hydrostatic and the pressure at depths $h_{1}$ and $h_{0}$ are assumed undisturbed, i.e. hydrostatic, by the change in pressure due to the volume change associated with the hydrate formation in the $\Delta L_{h}$ interval (Fig. 33). At the moment shown in Fig. 33 , the GWC is at a depth of $h_{0}-L_{w}$. As hydrate forms in the $\Delta L_{h}$ interval, GWC keeps moving upward to accommodate the transport of gas to the zone of hydrate formation above. Permeability to aqueous phase flow in the hydrate-bearing zone in the interval $\left[h_{1}, h_{1}+L_{h}\right]$ is denoted as $k_{w, h}$. The permeability to gas phase flow in the interval $\left[h_{0}-L_{w}, h_{0}-L_{t}\right]$ is denoted as $k_{g}$ and the permeability to aqueous phase flow below the depth of $h_{0}-L_{w}$ is shown as $k_{w}$. 


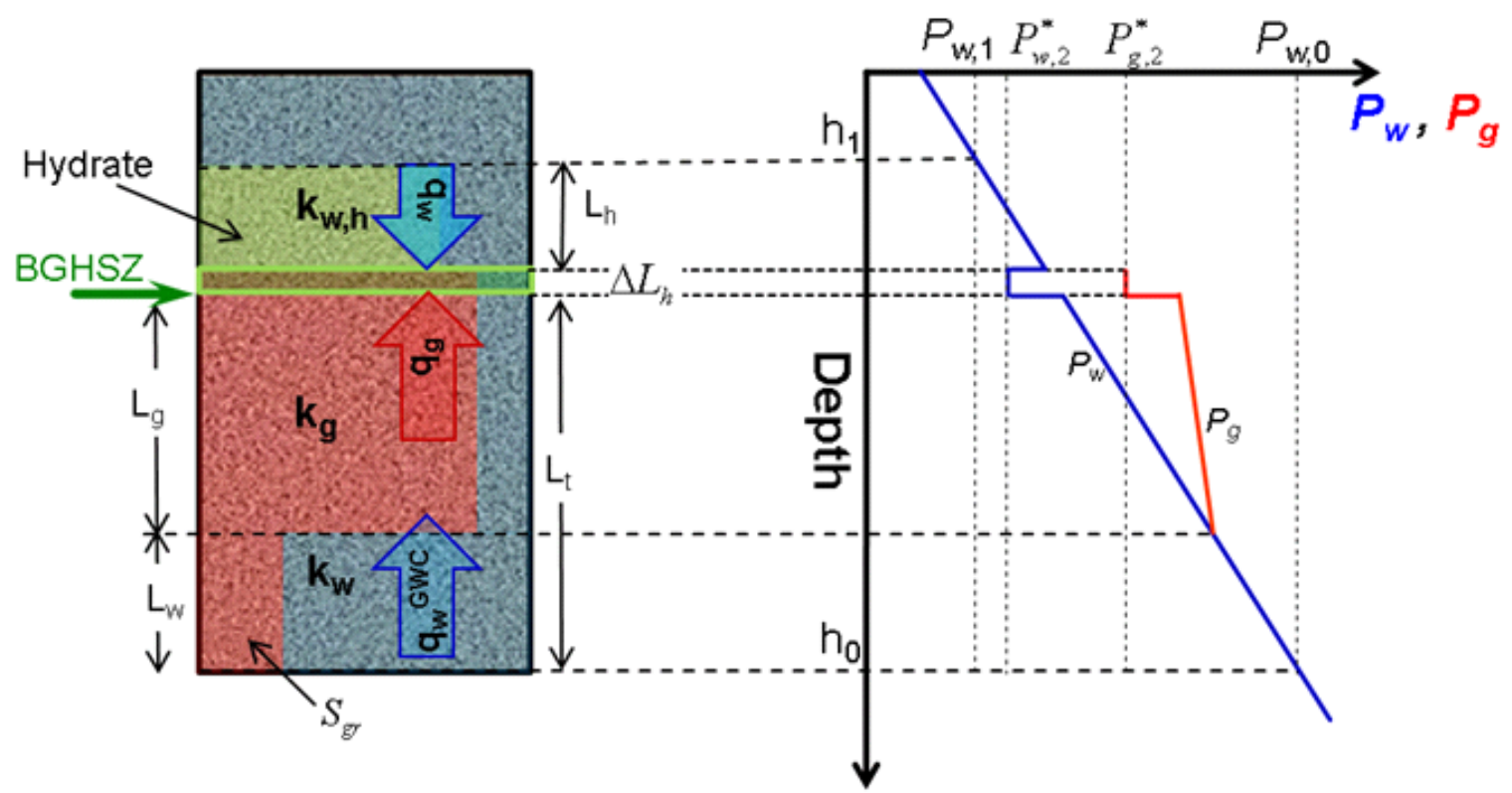

Fig. 33. The sand unit of Fig. 31 when the BGHSZ has moved down through a portion of the gas column. There is $L_{h}$ meters accumulation of hydrate above the current location of the BGHSZ. The GWC has moved upward (compare to Fig. 31) to depth $h_{0}-L_{w}$. The gas below the GWC has been transported upward and the gas saturation is $S_{g r}$. The phase pressure state inside the sediment is shown by a companion graph of pressure versus depth. The capillary pressure $P_{g}-P_{w}$ is zero at the GWC. The decrement in pressure at the BGHSZ indicates schematically the effect of the phase volume reduction accompanying hydrate formation.

The volume reduction associated with hydrate formation in the interval $\Delta L_{h}$ in Fig. 33 is assumed to decrease the aqueous phase pressure at the BGHSZ to a value $P_{w, 2}^{*}$ from its original hydrostatic value $P_{w, 2}=P_{w, 1}+\rho_{w} g L_{h}$. For an increment of hydrate, $d S_{h}$, the corresponding consumed amount of water and gas is calculated from Eqs. (43) and (44) (Behseresht \& Bryant 2011a).

$$
\begin{aligned}
& d S_{w}^{\text {used }}=\frac{N \bar{V}_{w}}{\bar{V}_{h}} d S_{h} \\
& d S_{g}^{\text {used }}=\frac{\bar{V}_{g}}{\bar{V}_{h}} d S_{h}
\end{aligned}
$$

For the initial fluid phase saturations, a total fluid compressibility can be calculated as:

$$
c_{t}=S_{w, i} c_{w}+S_{g, i} c_{g}
$$

If no fluid were to enter the interval $\Delta L_{h}$ then the pressure change, $d P$, associated with the consumed volume of gaseous and aqueous phase, is calculated from Eq. (46).

$d P=-\frac{1}{c_{t}} \frac{d V}{V_{\text {tot }}}=-\frac{1}{c_{t}} d S=-\frac{1}{c_{t}}\left(d S_{w}^{u s e d}+d S_{g}^{u s e d}-d S_{h}\right)$

Combining Eqs. (43), (44) and (46) gives:

$$
d P=\frac{-1}{c_{t}}\left(\frac{N \bar{V}_{w}+\bar{V}_{g}}{\bar{V}_{h}}-1\right) d S_{h}
$$


Therefore, the pressure, $P_{w, 2}^{*}$, after a decrement of $d S_{w}^{u s e d}$ and $d S_{g}^{u s e d}$ is calculated as:

$$
P_{w, 2}^{*}=\left[1-\frac{1}{P_{w, 2} \times c_{t}}\left(\frac{N \bar{V}_{w}+\bar{V}_{g}}{\bar{V}_{h}}-1\right) d S_{h}\right] P_{w, 2}=\left[1-\frac{1}{\left(P_{w, 1}+\rho_{w} g L_{h}\right) c_{t}}\left(\frac{N \bar{V}_{w}+\bar{V}_{g}}{\bar{V}_{h}}-1\right) d S_{h}\right]\left(P_{w, 1}+\rho_{w} g L_{h}\right)
$$

The reduction in pressure will induce flow in both gaseous and aqueous phases. From Darcy's law, the downward flow of water through the hydrate-bearing zone, $q_{w}$, would be:

$$
q_{w}=\frac{A k_{w, h}}{\mu_{w}}\left[\frac{P_{w, 1}-P_{w, 2}^{*}}{L_{h}}+\rho_{w} g\right]
$$

where $A$ is the cross sectional area, $g$ is the acceleration due to gravity and $\rho_{w}$ is density of aqueous phase. Substituting Eq. (48) in (49) after simplifying gives:

$$
q_{w}=A \frac{1}{c_{t}} \frac{N \bar{V}_{w}+\bar{V}_{g}}{\bar{V}_{h}} \frac{k_{w, h}}{L_{h} \mu_{w}} d S_{h}
$$

A check for the above equation is that if no hydrate forms, $d S_{h}=0$, and thus $q_{w}=0$, as is expected.

For the upward piston type flow of gas and water from below Darcy's law can be applied as:

$$
\left\{\begin{array}{l}
q_{w}^{G W C}=\frac{A k_{w}}{\mu_{w}}\left[\frac{P_{w, 0}-P}{L_{w}}-\rho_{w} g\right] \\
q_{g}=\frac{A k_{g}}{\mu_{g}}\left[\frac{P-P_{g, 2}^{*}}{L_{g}}-\rho_{g} g\right]
\end{array}\right.
$$

where $P$ is the flowing water pressure at the GWC and can be calculated from Eqs. (53) and (54). For the vertical flow of gaseous and aqueous phases we make the usual fractional flow theory assumption that:

$q_{w}^{G W C}=q_{g}$

Combining the two equations in Eq. (51) and the equation in Eq. (52) gives:

$$
\left\{\begin{array}{l}
P_{w, 0}-P=q_{g} \frac{L_{w}}{A \lambda_{w}}+\rho_{w} g L_{w} \\
P-P_{g, 2}^{*}=q_{g} \frac{L_{g}}{A \lambda_{g}}+\rho_{g} g L_{g}
\end{array}\right.
$$

Adding the two equations in Eq. (53) and simplifying gives:

$$
q_{g}=\frac{A}{\frac{L_{w}}{\lambda_{w}}+\frac{L_{g}}{\lambda_{g}}}\left[\left(P_{w, 0}-P_{g, 2}^{*}\right)-\left(\rho_{w} L_{w}+\rho_{g} L_{g}\right) g\right]
$$


Similar to $P_{w, 2}^{*}$, the corresponding pressure for the gas phase in the hydrate formation zone, $P_{g, 2}^{*}$, when an increment of hydrate, $d S_{h}$, forms can be expressed in terms of the equilibrium gas pressure, $P_{w, 2}+\Delta \rho_{w} g L_{g}$, and Eq. (47):

$$
\begin{aligned}
P_{g, 2}^{*} & =\left(1-\frac{1}{P_{g, 2} \times c_{t}} \frac{N \bar{V}_{w}+\bar{V}_{g}}{\bar{V}_{h}} d S_{h}\right) P_{g, 2} \\
& =\left(1-\frac{1}{\left(P_{w, 0}-\rho_{w} g L_{t}+\Delta \rho g L_{g}\right) c_{t}} \frac{N \bar{V}_{w}+\bar{V}_{g}}{\bar{V}_{h}} d S_{h}\right)\left(P_{w, 0}-\rho_{w} g L_{t}+\Delta \rho g L_{g}\right)
\end{aligned}
$$

Combining Eqs. (44), (54) and (55) gives the upward gas flow as:

$$
q_{g}=\frac{1}{c_{t}} \frac{N \bar{V}_{w}+\bar{V}_{g}}{\bar{V}_{h}} \frac{A}{\frac{L_{w}}{\lambda_{w}}+\frac{L_{g}}{\lambda_{g}}} d S_{h}
$$

We now have the rate of aqueous phase transport to the BGHSZ from above, Eq. (50), and the rate of gaseous phase transport to the BGHSZ from below, Eq. (56). We use these in the next section to determine $R_{v}$ as a function of the phase relative permeabilities.

\section{Calculating $R_{v}$ from Aqueous and Gaseous Phase Flows}

For this one-dimensional flow, Eq. (57) holds.

$$
\frac{q_{g}}{q_{w}}=\frac{u_{g}}{u_{w}}
$$

By definition, $R_{v}=\frac{q_{g}}{q_{g}+q_{w}}$ and thus:

$\frac{u_{g}}{u_{w}}=\frac{R_{v}}{1-R_{v}}$

Based on the Buckley-Leverett shock (Buckley \& Leverett 1942), shown in Fig. 32, the upward Darcy velocity of gaseous phase, $u_{g}$, can be expressed in terms of the water front speed, $d L_{w} / d t$ as in Eq. (59).

$u_{g}=\left(S_{g, i}-S_{g r}\right) \phi \frac{d L_{w}}{d t}$

where $\phi$ is the average porosity of the porous media through which the flow is taking place. Dividing Eq. (56) by Eq. (50) gives the left side of Eq. (57) from which $R_{v}$ can be calculated:

$$
\frac{u_{g}}{u_{w}}=\frac{k_{w}}{k_{w, h}} \frac{L_{h}}{M L_{t}+L_{w}(1-M)}
$$

where $M$ is the mobility ratio defined as:

$$
M=\frac{k_{w} / \mu_{w}}{k_{g} / \mu_{g}}
$$




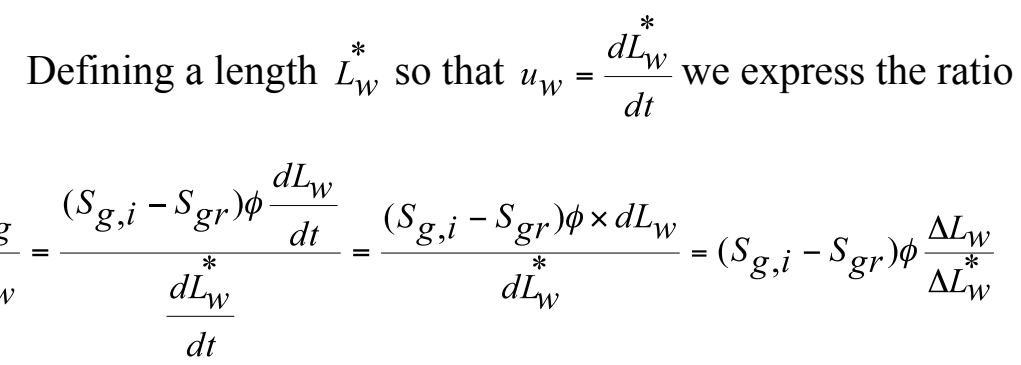$$
R_{v} \text { can be evaluated from Eqs. (58) and (62) as: }
$$$$
R_{v}=\frac{\left(S_{g, i}-S_{g r}\right) \phi \Delta L_{w}}{\left(S_{g, i}-S_{g r}\right) \phi \Delta L_{w}+\Delta L_{w}^{*}}
$$

Combining Eqs. (60) and (62) gives:

$$
\begin{aligned}
& \frac{\left(S_{g, i}-S_{g r}\right) \phi \times d L_{w}}{d L_{w}^{*}}=\frac{k_{w}}{k_{w, h}} \frac{L_{h}}{M L_{t}+L_{w}(1-M)} \\
& \Rightarrow \frac{d L_{w}}{d L_{w}^{*}}=\frac{\xi}{M L_{t}+L_{w}(1-M)}
\end{aligned}
$$

where,

$$
\xi=\frac{k_{w}}{k_{w, h}} \frac{L_{h}}{\left(S_{g, i}-S_{g r}\right) \phi}
$$

The dynamic quantity in is the thickness of the hydrate zone $L_{h}$ and the overall permeability of the hydrate-bearing interval, $k_{w, h}$. Thus Eq. (64) relates the rate at which the gas-water contact moves to the thicknesses of the hydrate zone, the remaining gas column $L_{t}$ and the residual gas zone $L_{w}$. The influence of the relative permeability curves appears in the mobility ratio $M$ because of co-current flow in the gas column. These curves also determine the ratio of aqueous phase permeability below the GWC, $k_{w}$, to the aqueous phase permeability in the hydrate-bearing sediment, $k_{w, h}$, which affects the magnitude of in Eq. (66). Rearranging and integrating Eq. (65) gives:

$$
\int_{0}^{\Delta L_{w}}(1-M) L_{w} d L_{w}+\int_{0}^{\Delta L_{w}} M L_{t} d L_{w}=\int_{0}^{\Delta L_{w}^{*}} \xi d L_{w}^{*}
$$

Hence,

$$
\Delta L_{w}^{*}=\frac{1}{\xi}\left[\frac{(1-M)}{2} \Delta L_{w}^{2}+M L_{t} \Delta L_{w}\right]
$$

Based on the above discussion, when the BGHSZ moves down from $h_{1}+L_{h}$ to $h_{1}+L_{h}+\Delta L_{h}$, the following algorithm can be used to calculate $R_{v}$ and thus hydrate saturation, $S_{h}$, in the $\Delta L_{h}$ interval newly introduced in the GHSZ:

a. Start with an initial guess for $R_{v}=R_{v, t e m p}$ 
b. Calculate $\Delta L_{w}$ from Eq. (34).

The required pore volume of gas required to be transported into the $\Delta L_{h}$ interval, $\Delta V_{g, d}$, which is a function of $R_{v}$ can be calculated from Eq. (40). This amount of gas will be provided through sweeping $\Delta L_{w}$ meters of the gas column by the water front from below; therefore:

$$
A \phi\left(S_{g, i}-S_{g r}\right) \Delta L_{w}=A \phi \Delta L_{h} \Delta V_{g, d}
$$

Hence,

$$
\Delta L_{w}=\frac{\Delta V_{g, d}}{S_{g, i}-S_{g r}} \Delta L_{h}
$$

c. Calculate $\Delta L_{w}^{*}$ from Eq. (68).

d. Update the value of $R_{v, \text { temp }}$ using Eq. (63).

e. If $\left|R_{v} / R_{v, \text { temp }}-1\right|>0.001$ go to Step a.

f. $R_{v}=R_{v, \text { temp }}$ is the final calculated $R_{v}$ for forming hydrate in the interval $\Delta L_{h}$.

From the calculated value of $R_{v}$, the final hydrate saturation in the $\Delta L_{h}$ new hydrate-bearing interval is calculated from Eq. (38) Hence this model provides an a priori method of estimating $R_{v}$, the gas phase fraction of the total phase volume arriving at the BGHSZ. We use the above algorithm to predict the hydrate saturation distribution, given the initial distribution of phase saturations (before descent of BGHSZ), the $P$ and $T$ of the GHSZ, the residual gas saturation, the relative permeabilities of gaseous and aqueous phases, and the aqueous phase relative permeability when hydrate occupies sediment pore space.

$$
k_{w, h}=\frac{L_{h}+\Delta L_{h}}{\frac{L_{h}}{k_{w, L_{h}}}+\frac{\Delta L_{h}}{k_{w, \Delta L_{h}}}}
$$

There are several models to estimate the aqueous phase permeability in the presence of hydrate. Here, we assume that hydrate fills center of the pores and thus a pore-filling permeability model applies. Let the permeability of non-hydrate-bearing sediment in the $\Delta L_{h}$ interval be denoted $k$. Based on the pore-filling model (Kleinberg et al. 2003), if the hydrate saturation in the interval is $S_{h}$, the permeability to aqueous phase of the hydrate-bearing $\Delta L_{h}$ interval, $k_{w, \Delta L h}$ is calculated from Eq. (72).

$$
k_{w, \Delta L_{h}}=\frac{\left(1-S_{h}\right)^{n+2}}{\left(1+\sqrt{S_{h}}\right)^{2}} k
$$

where $n$ is the hydrate saturation exponent. Neglecting the effect of capillary pressure, the saturation exponent increases from $n=0.4$ at $S_{h}=0.1$ to unity at $S_{h}=1$ (Spangenberg 2001; Kleinberg et al. 2003).

\section{Application of Transport Model to Mt Elbert}

The above model is applied on the D sand unit of Mt. Elbert well. First, the profile of absolute permeability of the sand unit versus depth is established. For the grain size distribution in Mt. Elbert well, Eq. (73) can be used to estimate the permeability at each depth as a function of grain size distribution at that depth: 
$k=6 \times 10^{-4} \log \left(500 \frac{D_{10}}{D_{60}}\right) D_{10}^{2}$

where, $D_{10}$ and $D_{60}$ are the $10^{\text {th }}$ and $60^{\text {th }}$ percentile of the grain diameter distribution, respectively (Behseresht \& Bryant 2011b). Figure 34 shows the estimated permeability versus depth in the D sand unit based on the grain size distribution shown in Fig. 29a.

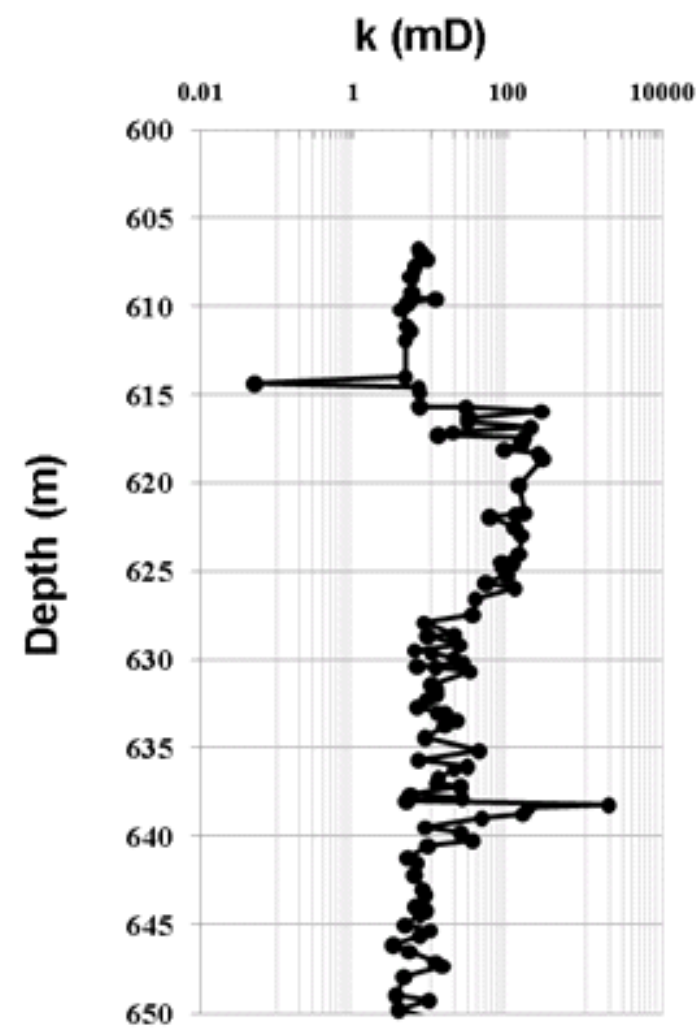

Fig. 34. Estimated permeability from Eq. (73) based on grain size distribution data for the D sand unit in Mt. Elbert well. Grain size variation versus depth is shown in Fig. 29a.

To calculate the mobility ratio, $M$, of aqueous to gaseous phase, for the upward piston type co-current flow, the end-point relative permeabilities of aqueous and gaseous phase are needed. Here a typical pair of end-point relative permeability values for water-wet sediments is used: 0.3 for water and 0.7 for methane, as shown on the typical relative permeability curve for water-wet sediment in Fig. 35. Dynamic methane viscosity, $\mu_{g}$, is estimated to be $1.2 \times 10^{-5} \mathrm{~Pa} . \mathrm{s}$ at the current depth-averaged pressure and temperature of the sand unit $\left(P=6.5 \mathrm{MPa}\right.$ and $\left.T=2^{\circ} \mathrm{C}\right)$ (Huang et al. 1966). At the same thermodynamic condition, viscosity of water, $\mu_{w}$, is estimated to be $1.7 \times 10^{-3}$ Pa.s (Stanley \& Batten 1969). Thus $M$ is $3 \times 10^{-3}$. Note that due to the hundred-fold difference in gas and aqueous phase viscosities, we have $M<<1$ for typical ratios of end point relative permeabilities. 


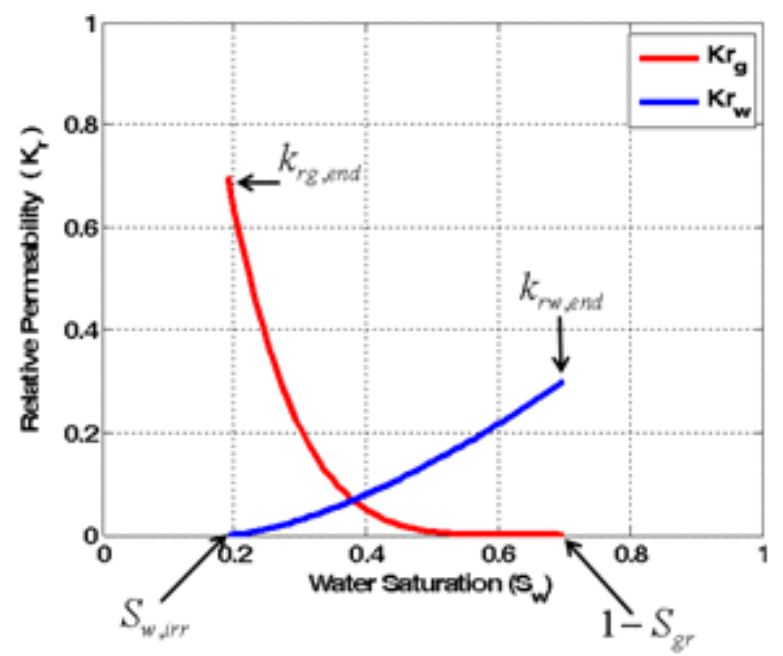

Fig. 35. Typical relative permeability curves for a water-wet sediment; red represents the relative permeability of gaseous phase and blue represents that of the aqueous phase. The end point relative permeabilities, along with the end point saturations are shown on the graph.

We apply the above model (Fig. 31), starting with the same initial gas saturation profile as that estimated by Behseresht and Bryant (2011b) based on the capillary entry pressure profile calculated from the grain size distribution at each depth. The initial gas saturation profile in the D sand unit can be approximated by that shown in Fig. 31 having $h_{0}=650 \mathrm{~m}, h_{1}=614 \mathrm{~m}, S_{w, i r r}=$ 0.18 and $S_{g r}=0.3$ (Behseresht et al. 2009).

Figure 36 a shows the calculated $R_{v}$ versus depth with a solid black line as the BGHSZ moves down through the methane/water bearing sediment. The reason that $R_{v}$ values increase with depth from the upper part of the unit is that as the BGHSZ moves downward and the length of hydratebearing sediment, $L_{h}$, increases, the overall permeability of the hydrate bearing sediment, $k_{w, h}$ decreases. This reduces the water flow rate from above which is equivalent to an increase in $R_{v}$. The $R_{v}$ in the imbibed zone below $630 \mathrm{~m}$, swept by water to the end-point saturation $1-S_{g r}$, is zero. This is because when BGHSZ descends into the residual gas zone, no more gas is available to flow upwards as all gas saturation below BGHSZ is at residual. Examination of Eqs. (63) and (68) shows that the calculated $R_{v}$, and hence the predicted hydrate saturation profile, is independent of the porosity, $\phi$. In fact, for a given initial gas water saturation, the ratio $k_{w} / k_{w, h}$ is the main parameter governing $R_{v}$ under a fixed thermodynamic condition (i.e. at fixed $T$ and $P$, hence $\bar{V}_{g} / N \bar{V}_{w}=$ const ). 
(a)

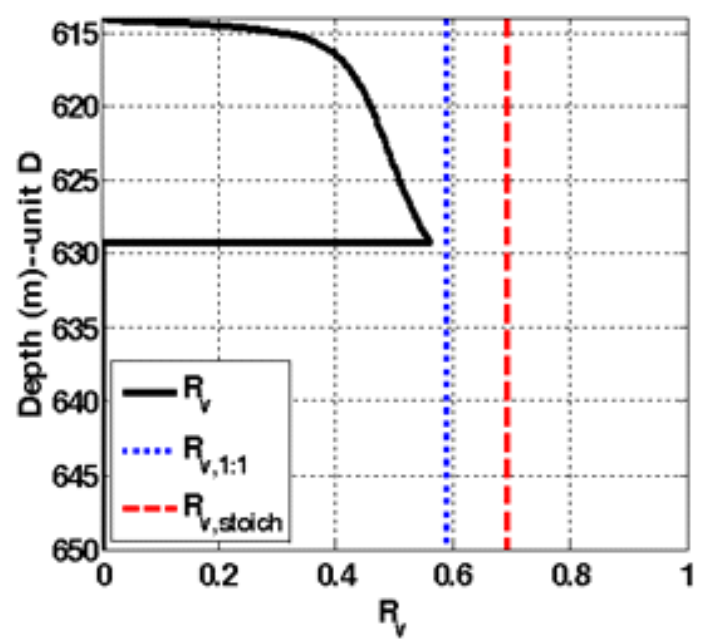

(b)

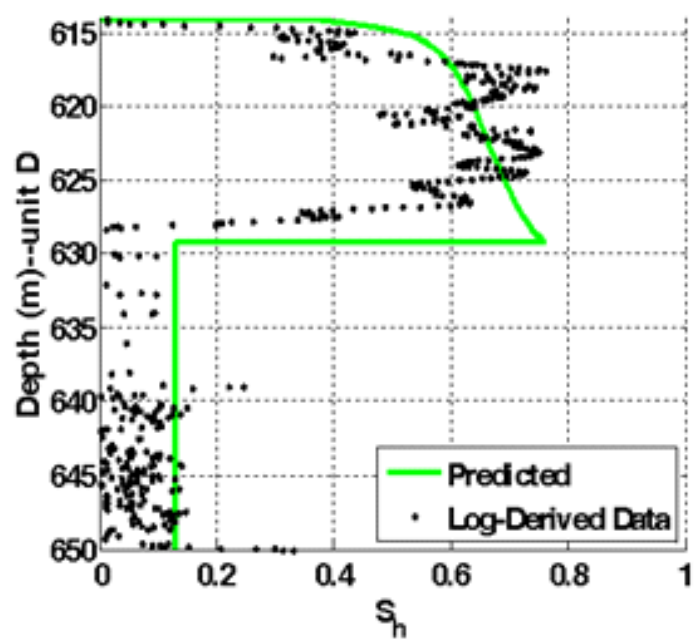

Fig. 36. Application of the pressure-driven fluid transport model to the D sand unit in Mt. Elbert well using an end-point $k_{r, w}=0.3 . k_{w, h}$ is determined from Eq. (71). (a) $R_{v}$ versus depth shown in solid black line. $R_{v}$ is zero below $629 \mathrm{~m}$ because the gas saturation dropped to residual there as gas moved upward to the BGHSZ. The values of $R_{v, 1: 1}$ and $R_{v, \text { stoich }}$ are shown in dotted line and dashed line, respectively. (b) Predicted hydrate saturation profile in green solid line. Dots represent the log-derived hydrate saturation data (Rose et al. 2011).

The calculated value of $R_{v}$ determines the hydrate saturation at each depth. Figure 36b shows the predicted hydrate saturation profile along with the log-derived hydrate saturation data of the D sand unit in Mt. Elbert well (Rose et al. 2011). Note that the hydrate saturation profile shown in green solid line (Fig. 36b) evolved from an initially uniform gas saturation profile with $S_{g, i} \approx 1-S_{w, \text { irr }}=0.8$ filling almost the entire D sand unit. The agreement with the measured values of $S_{h}$ is reasonable. The trend of increasing $S_{h}$ from $614 \mathrm{~m}$ to $620 \mathrm{~m}$ is evident, as is the small but nonzero $S_{h}$ between $630 \mathrm{~m}$ and $650 \mathrm{~m}$.

Applying the model on D sand unit in Mt. Elbert well with a smaller end-point relative permeability for aqueous phase compared to that in Fig. $35, k_{w}=0.1$, predicts a longer hydrate column but at smaller saturation as shown in Fig. 37. The reason is that the smaller aqueous phase end-point relative permeability slows the rate at which gas can move to the BGHSZ, causing $R_{v}$ to be smaller, and hence $S_{h}$ to be smaller. The mass of gas converted to hydrate is the same as in Fig. 36, so the smaller $S_{h}$ requires a longer column in the upper hydrate-bearing zone than in Fig. 36. 
(a)

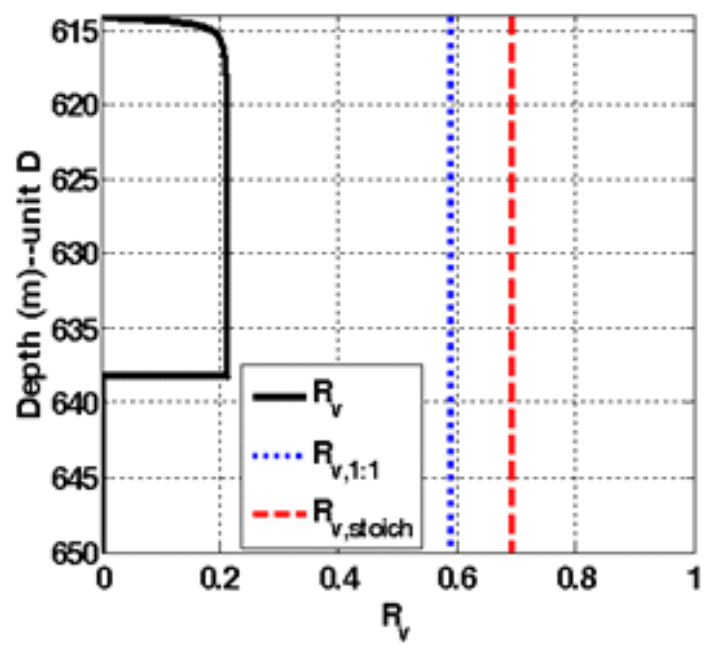

(b)

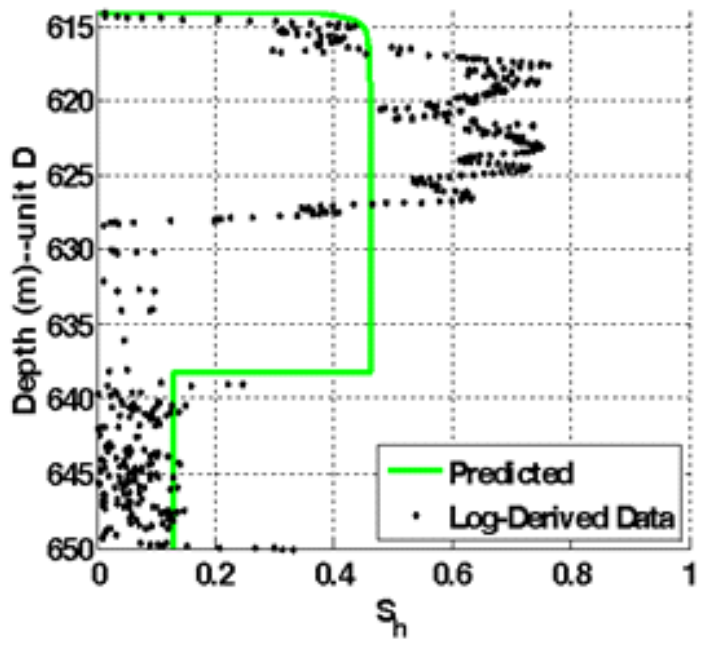

Fig. 37. Application of the pressure-driven fluid transport model to the D sand unit in Mt. Elbert well using an end-point $k_{r, w}=0.1$ yields smaller $R_{v}$ and correspondingly thicker upper zone of hydrate at a smaller saturation than in Fig. 36. (a) $R_{v}$ versus depth shown in solid black line. (b) Predicted hydrate saturation profile in green solid line. Dots represent the log-derived hydrate saturation data (Rose et al. 2011).

Figure $38 \mathrm{~b}$ shows the predicted hydrate saturation profile when the end-point relative permeability to aqueous phase is changed from 0.3 (in Fig. 35) to 0.5 . The larger value enables more rapid transport of gas to the BGHSZ, causing $R_{v}$ to increase. This results in larger hydrate saturations. This reduces the aqueous phase permeability in the hydrate-bearing sediment, causing $R_{v}$ to increase further. In the present model, this feedback leads to complete conversion of aqueous phase saturation to hydrate, yielding $S_{h}=1$ in the upper part of the column. This prevents subsequent transport of water from above the BGHSZ. Thus the hydrate saturation below this point corresponds only to converting the existing initial water saturation, and a large gas saturation is predicted to remain unconverted (pink box in Fig 38). Neither the hydrate saturation profile nor the presence of a gas saturation is consistent with observations.

(a)

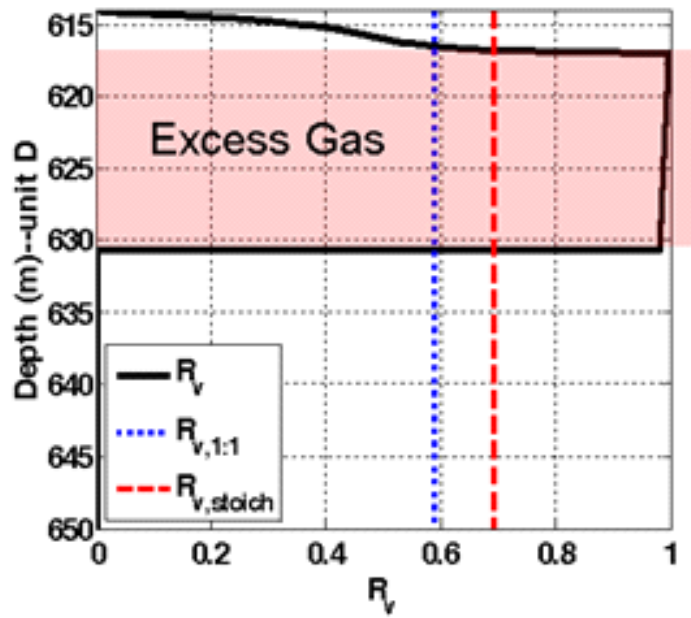

(b)

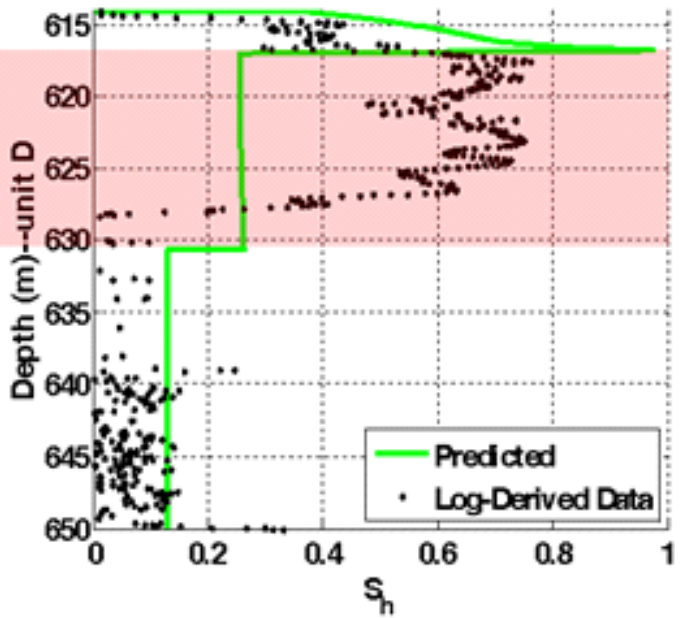

Fig. 38. Applying the fluid model along with the stoichiometric model on the D sand unit in Mt. Elbert well using an end-point $k_{r, w}=0.5$ yields a qualitatively different behavior than Figs. 36 and 37 . (a) $R_{v}$ versus 
depth shown in solid black line. Calculated $R_{v}$ from the flow model exceeds $R_{v, s t o i c h}$ in part of the sand unit which is shown with a red fill. (b) Predicted hydrate saturation profile in green solid line. The zone identified with red fill contains hydrate and gas only which is in contradiction to the observed profile in field having hydrate and water only.

\section{Discussion of Pressure-Driven Transport Model}

Comparing the above figures it is evident that Mt. Elbert agrees more with $k_{r w, e n d}=0.3$, Fig. 36. Therefore, the proposed model is consistent with typical relative permeability curves and typical end-point relative permeabilities. However, the model is sensitive to the endpoint aqueous phase effective permeability $k_{r w, \text { end }}$. In fact, the ratio of $k_{r w, \text { end }} / k_{w, h}$ is also crucial to the behavior of the model. The same sensitivity shown in Figs. 37 and 38 would also apply if the value of permeability to aqueous phase in the hydrate-bearing sediment $k_{w, h}$ were to change by a factor of two. Laboratory experiments reported in the literature suggest that this permeability is highly sensitive to hydrate saturation, and in any case it will also depend strongly on the variation in grain size within the hydrate-bearing sediment. Moreover the model contains a feedback loop, in that larger $k_{r w, \text { end }}$ yields larger hydrate saturations, which cause smaller values of $k_{w, h}$. This is the cause of the increase in $S_{h}$ with depth between 614 and $629 \mathrm{~m}$ in Fig. 36, and between $614 \mathrm{~m}$ and $617 \mathrm{~m}$ in Fig. 38. The hydrate saturation profiles predicted for smaller or larger values of $k_{r w, e n d} / k_{w, h}$ shown in Figs. 37 and 38 respectively, are not observed at Mt Elbert (M. W. Lee \& T. Collett 2011).

Numerous hydrate accumulations in the Alaska North Slope exhibit saturation profiles qualitatively similar to Mt Elbert. Yet the pressure-driven transport model yields the behavior observed at Mt Elbert only for a rather narrow range of choices of $k_{r w, e n d} / k_{w, h}$. The range of choices seems particularly narrow considering the likely range of values in different accumulations. For these reasons, it seems unlikely that pressure-driven flow is responsible for supplying gas and aqueous phases to the hydrate stability zone during conversion. Physically, the problem with this model is that pressure-driven flow cannot supply aqueous phase from below the BGHSZ. The gas phase is supplied from below the BGSHZ at a rate proportional to $k_{r w, e n d}$ while the aqueous phase must be supplied from above at a rate proportional to $k_{w, h}$. The ratio $k_{r w, e n d} / k_{w, h}$ fixes the relative rates of gas and aqueous phase transport. These rates must be rather finely balanced to yield the observed saturation profiles.

The pressure-driven model neglects an important aspect of fluid flow in porous media, especially when water saturation approaches $S_{w, i r r}$ : capillary pressure gradients causing an extra water suction to keep the aqueous phase connected, i.e. $S_{w} \geq S_{w, i r r}$. This is in analogy with the observed induced water suction during frost heave formation, which keeps the aqueous phase connected for continued heave formation (Penner 1959; Hermansson \& Spencerguthrie 2005). Formation of hydrate is analogous in several respects to frost heave formation (Cook et al. 2008; J. Y. Lee et al. 2011; Rees et al. 2011). Once the aqueous phase saturation becomes close to the $S_{w, i r r}$, further decrement of the aqueous phase saturation due to hydrate formation would cause a large capillary pressure gradient. This would transport aqueous phase to the zone to counteract the decrement in saturation. Because the gradient $d P_{c} / d S_{w}$ is largest near $S_{w, i r r}$, this mechanism is likely to prevent $R_{v}$ from increasing beyond $R_{v, 1: 1}$. Figure 39 shows the model prediction for the same parameters as in Fig. 38, but assuming $R_{v}$ cannot exceed $R_{v, 1: 1}$. The predicted hydrate saturation matches the log-derived data and predicts an excess water situation (having hydrate and water only) which is expected. 
(a)

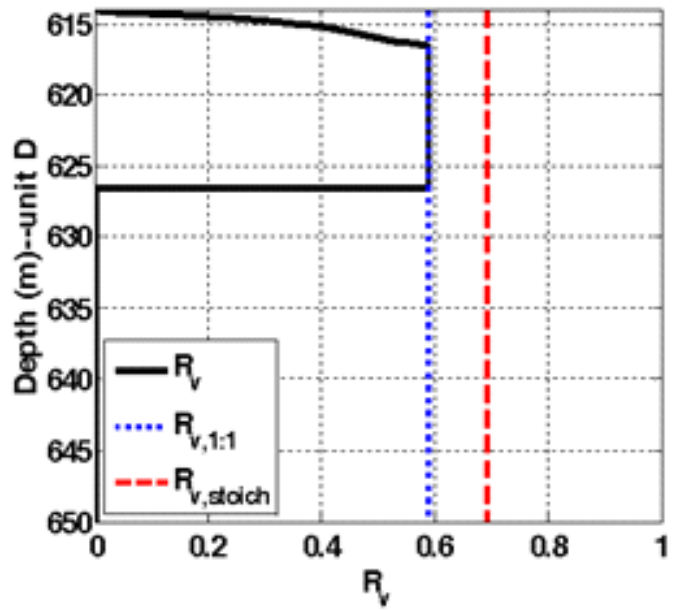

(b)

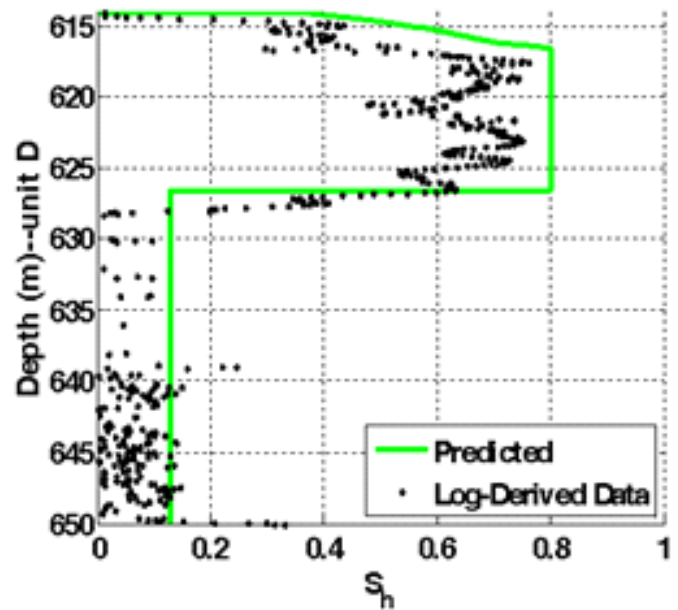

Fig. 39 Applying a constrained pressure-driven fluid transport model along with the stoichiometric model on the D sand unit in Mt. Elbert well using an end-point $k_{r, w}=0.5$; the constraint is that $R_{v}$ is not allowed to exceed the value $R_{v, 1: 1}$. The predicted hydrate saturation profile overestimates the measurements in the upper part of the unit and yields a slightly thinner accumulation. The small hydrate saturation below $627 \mathrm{~m}$ is the result of converting residual gas saturation to hydrate.

Of course the constraint of $R_{v}$ is entirely ad hoc. The fact that overriding the pressure-driven flow model in this way yields a saturation profile close to what is observed raises the interesting hypothesis that capillarity-driven transport is a more general means of accounting for the behavior during conversion to hydrate. This observation is the motivation for the next section of this report.

\section{Summary of Applicability of Pressure-Driven Transport Model for Conversion of Gas Reservoirs to Hydrate}

The conversion of a gas accumulation into hydrate reduces the volume occupied by fluid phases and thus drives the flow of fluid phases toward the zone of hydrate formation. Assuming this flow is driven by gradient in potential has significant implications for the applicability of the model. In particular, a co-current flow of gas and aqueous phases from below a descending base of gas hydrate stability zone (BGHSZ) upward into the hydrate stability zone (GHSZ) cannot provide the required amount of water for hydrate formation. This is because the rising gas phase forms a sharp front, in accord with fractional flow theory, ahead of which only gas flows. Thus the pressure-driven model must transport water to the GHSZ from above. That is, water moves down into the GHSZ through accumulated hydrate from overlying aquifers, while gas rises to the GHSZ from the remaining gas reservoir.

With suitable choices of the key transport coefficients (effective permeability to aqueous phase at the residual gas endpoint, and effective permeability to aqueous phase of sand containing large hydrate saturation), the countercurrent transport of gas and aqueous phases provides the large volumes of both components needed to form large hydrate saturations observed in Arctic sediments. The large saturations occur only in the upper portion of the original gas accumulation, with the thickness and the saturation depending on the ratio of aqueous phase end point relative permeability to aqueous phase permeability in hydrate-bearing sediment. A column of small hydrate saturations (10-15\%) forms below the upper zone; this lower zone is where residual gas phase saturation was established as gas flowed upward to the 
GHSZ and aqueous phase imbibed. During hydrate formation in the imbibed zones the only phase flowing into the GHSZ due to the volume change is the aqueous phase.

For plausible endpoint relative permeabilities for aqueous phase and for a reasonable choice of a relationship between water effective permeability and hydrate saturation, the model prediction agrees with behavior observed in the Mt. Elbert well. However the model prediction is highly sensitive to the ratio of these transport coefficients. Small variations in grain size distribution, end point relative permeability, or hydrate saturation dependence yield large variations in predicted hydrate profiles, including qualitatively different behavior, e.g. preservation of a large gas phase saturation. Thus the pressure-driven fluid transport model is unlikely to provide a robust explanation of the mechanism of conversion. Instead it offers useful insight into the likely role of capillarity-driven transport, which is discussed in the next section of this report.

\section{Gas Reservoir Conversion to Hydrate: Role of Capillarity-Driven Fluid Phase Transport at Bed-Scale}

The preceding section shows that pressure-driven, viscous-dominated flow is unlikely to account for observed hydrate saturation distributions at Mt Elbert or elsewhere in the subpermafrost accumulations. In this section we generalize the fluid flow model to account for capillarity-driven transport. For this application, saturation gradients provide the driving force. The model is otherwise the same as described in the preceding section. Here we recapitulate the main features of that model and include the relevant terms for capillarity-driven transport.
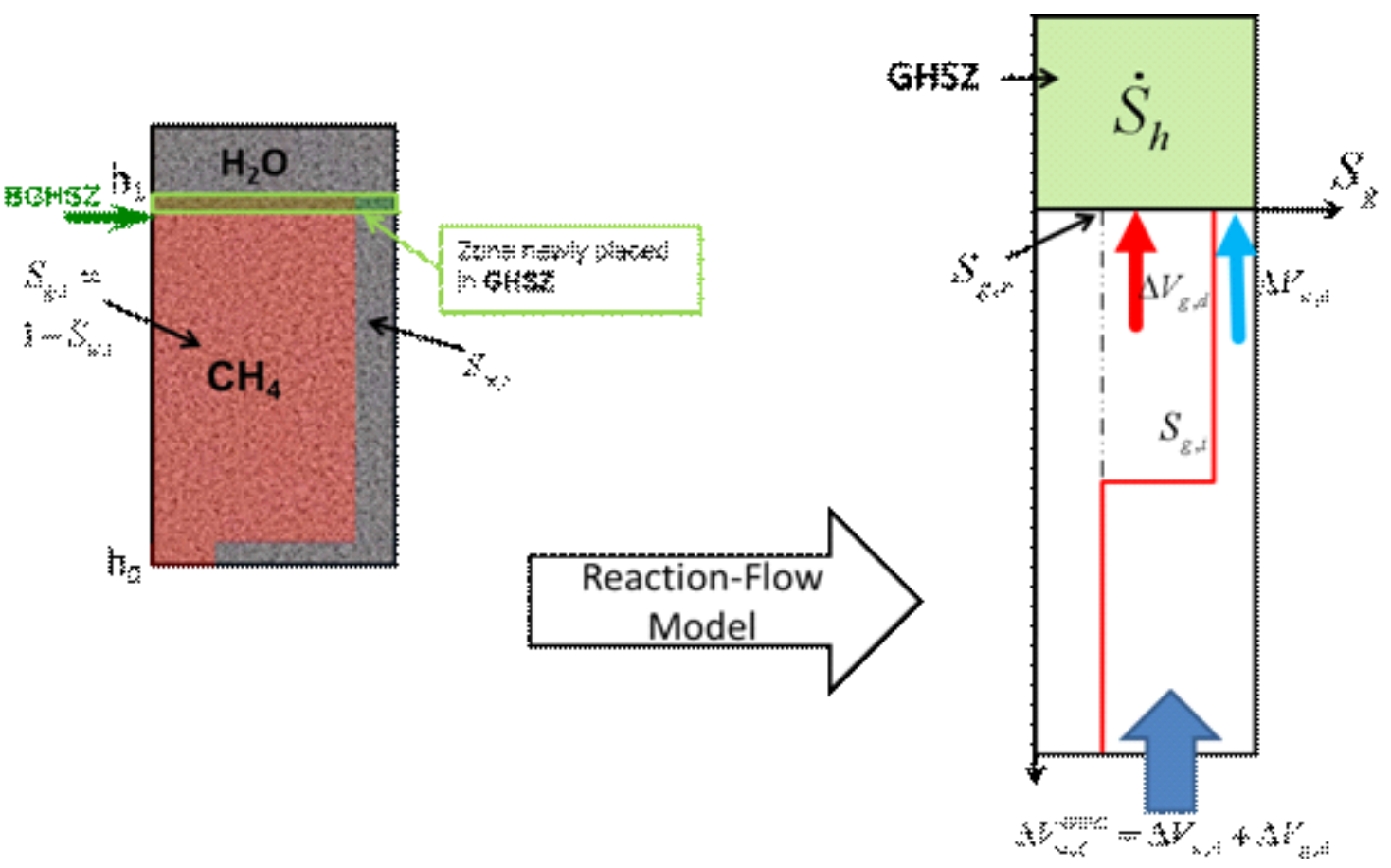

Fig. 40. Schematic of fluid flow induced by hydrate formation during descent of BGHSZ.

Assume that flow of gaseous and aqueous phases occurs only from below in response to the generation of hydrate, as shown in Fig. 40. The flow of gaseous, $u_{g}$, and aqueous phase, $u_{w}$, are as follows: 


$$
\begin{aligned}
& u_{w}=-k \lambda_{w}\left(\frac{\partial P_{w}}{\partial z}-\rho_{w} g\right) \\
& u_{g}=-k \lambda_{g}\left(\frac{\partial P_{g}}{\partial z}-\rho_{g} g\right)
\end{aligned}
$$

where,

$$
\lambda_{i}=\frac{k_{r, i}}{\mu_{i}} \quad ; i=w, g
$$

As mentioned in earlier sections, the difference between the gaseous phase pressure, $P_{g}$, and aqueous phase pressure, $P_{w}$, is known as capillary pressure, $P_{c}$. Therefore, the gradients of the two phase pressures are related as follows:

$\frac{\partial P_{g}}{\partial z}=\frac{\partial P_{w}}{\partial z}+\frac{\partial P_{c}}{\partial z}$

Defining $u_{T}$ as the total flux of gaseous and aqueous phases combined with Eq. (77) gives:

$$
u_{T}=u_{w}+u_{g}=-k \lambda_{w}\left(\frac{\partial P_{w}}{\partial z}-\rho_{w} g\right)+-k \lambda_{g}\left(\frac{\partial P_{w}}{\partial z}+\frac{\partial P_{c}}{\partial z}-\rho_{g} g\right)
$$

Eq. (78) can be further simplified as follows:

$$
u_{T}=-k\left(\lambda_{w}+\lambda_{g}\right) \frac{\partial P_{w}}{\partial z}+k\left(\lambda_{g} \rho_{g}+\lambda_{w} \rho_{w}\right) g-k \lambda_{g} \frac{\partial P_{c}}{\partial z}
$$

Solving for the aqueous phase pressure gradient from Eq. (79), and substituting in Eq. (74) gives:

$$
u_{w}=\underbrace{\frac{\lambda_{w}}{\lambda_{w}+\lambda_{g}} u_{T}}_{\text {Viscous }}+k \underbrace{\frac{\lambda_{w} \lambda_{g}}{\lambda_{w}+\lambda_{g}} \frac{\partial P_{c}}{\partial z}+k \frac{\lambda_{w} \lambda_{g}}{\lambda_{w}+\lambda_{g}}\left(\rho_{w}-\rho_{g}\right) g}_{\text {Capillary }} \underbrace{+\lambda}_{\text {Gravity }}
$$

As indicated in Eq. (80), three contributions to the water flux are viscous (pressure driven), capillary (saturation gradient driven) and gravity (buoyancy).

Furthermore, the mass balance equations for both gaseous and aqueous phases are as follows:

$$
\begin{aligned}
& \phi \frac{\partial}{\partial t} S_{w}+\frac{\partial}{\partial z} u_{w}+\alpha_{w} \dot{S}_{h}=0 \\
& \phi \frac{\partial}{\partial t} S_{g}+\frac{\partial}{\partial z} u_{g}+\alpha_{g} \dot{S}_{h}=0
\end{aligned}
$$

$\alpha_{w}$ and $\alpha_{g}$ are stoichiometric conversions relating hydrate formation rate to consumption rate of water and methane; for simplicity we assume pure phases so that aqueous and gaseous phase saturations are synonymous with water component and methane component concentrations, respectively: 
$\alpha_{w}=\phi \frac{N \bar{V}_{w}}{\bar{V}_{h}}$

$\alpha_{g}=\phi \frac{\bar{V}_{g}}{\bar{V}_{h}}$

where $\phi$ is porosity and $\bar{V}_{w}, \bar{V}_{h}$ and $\bar{V}_{g}$ are the molar volumes of water, hydrate and methane, respectively.

Summation of Eqs. (81) and (82) gives:

$\phi \frac{\partial}{\partial t}\left(S_{w}+S_{g}\right)+\frac{\partial}{\partial z}\left(u_{w}+u_{g}\right)+\left(\alpha_{w}+\alpha_{g}\right) \dot{S}_{h}=0$

Substituting $S_{w}+S_{g}=1-S_{h}$ in Eq. (85) and simplifying gives:

$(\alpha-\phi) \dot{S}_{h}+\frac{\partial}{\partial z} u_{T}=0$

where $\alpha=\alpha_{w}+\alpha_{w}$.

The final system of equations is as follows:

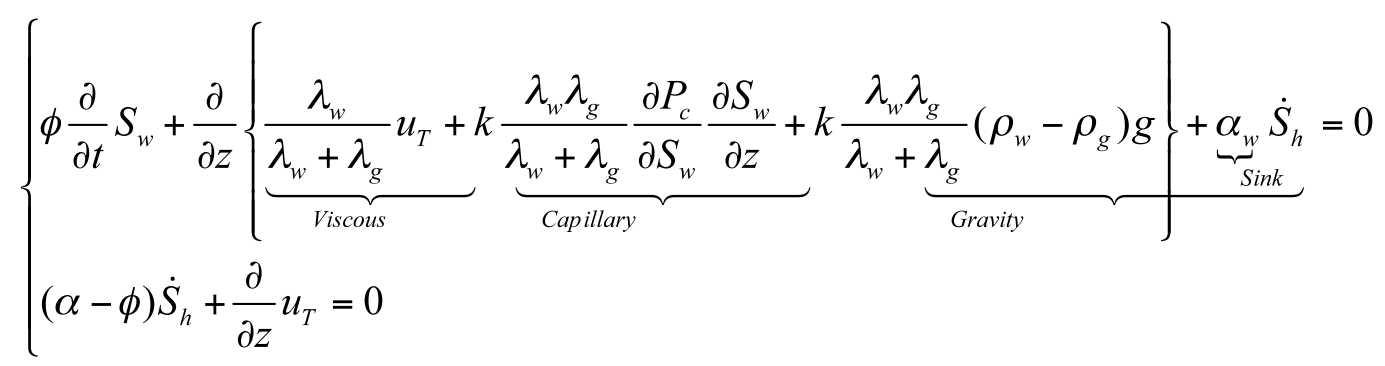

The solution to Eq. 87 depends on several rock and fluid properties: the relative permeability curves (for the phase mobilities), the capillary pressure curve (for the gradient of capillary pressure vs. saturation) and phase densities (for buoyancy). All flows are driven by rate of hydrate formation, which is prescribed as a boundary condition in the model. The domain includes the gas reservoir plus a finite volume of aquifer below the gas/water contact, which is assumed to be at constant pressure equal to hydrostatic. The top of the gas reservoir is assumed to be sealed to fluids (both gas and water).

The model assigns the rate of hydrate formation in a finite volume of sediment. It computes fluxes in the gas/water region below the BGHSZ and fluxes within the GHSZ. The latter depends on the permeability to fluids in the presence of hydrate, for which we use an empirical correlation. Note that $\dot{S}_{h}=f(z, t)$ is a function of space and time. In fact we impose a moving boundary condition on the above system of equations to mimic the descent of the BGHSZ happened in response to surface temperature cooling in the Arctic.

$$
\dot{S}_{h}(z, t)=\left\{\begin{array}{lll}
\dot{S}_{h} & ; & z \leq z_{B G H S Z} \\
0 & ; & z>z_{B G H S Z}
\end{array}\right.
$$

where $z_{B G H S Z}$ is the depth of the BGHSZ which in turn is a function of time, $z_{B G H S Z}=f(t)$. 
In the following results, the applied rates of descent of the BGHSZ are assumed constant. It is also possible to assign variable, with time $t$, rates of descent of the BGHSZ. The main output of the model is profiles of flux and saturation for each phase. The three contributions to the flux of each fluid phase can also be extracted.

\section{Illustration of the pressure-driven and capillarity-driven model}

In this section we consider an initial gas reservoir through which gaseous and aqueous phase saturations are distributed based on capillarity/gravity equilibrium. Then a top section of the gas reservoir is placed inside the GHSZ and the emerged gaseous and aqueous phase fluxes are predicted. We show that the rate of hydrate formation multiplied by the rate of descent of the BGHSZ plays a key role in determining the final hydrate saturation as well as the final state of the system, i.e. hydrate coexists with aqueous phase vs hydrate coexists with gas phase. To simplify the interpretation of the model, we fix the level of the BGHSZ at 5 meters below the seal (top of the gas column) and use three different magnitudes for the rate of hydrate formation, $\dot{S}_{h}$.

In Cases 1, 2 and 3 the capillarity and relative permeability characteristics of the host sediment are fixed as shown in Fig. 41 and 42.

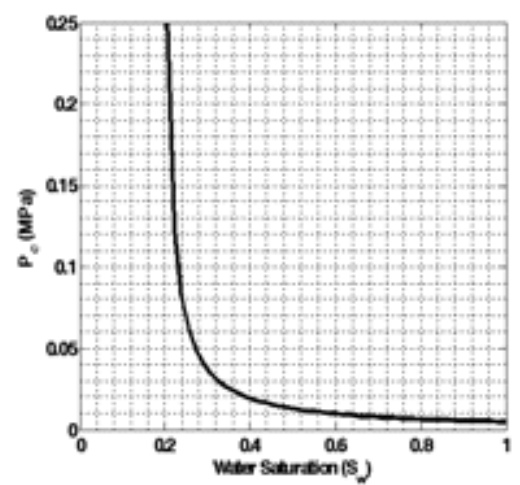

Fig. 41. Rock properties used in capillarity-driven flow model: Capillary pressure vs water saturation.

In case 4 , the same rate of hydrate formation as that in Case 2 is used, but different capillarity and relative permeability characteristic, as shown below, are used. The host sediment in Case 4 is more water-wet and exhibits a sharper capillary percolation compared to that of Cases 1,2 and 3.

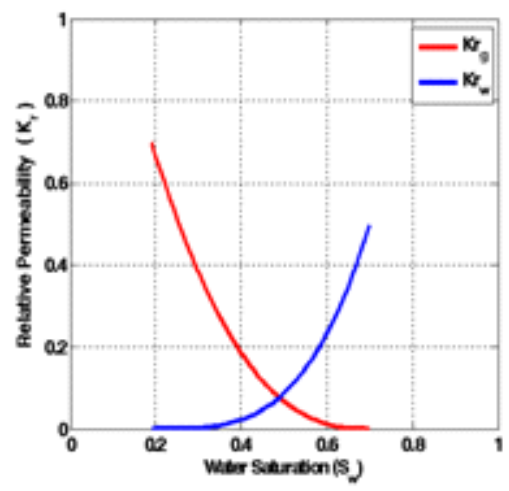

Fig. 42. Rock properties used in capillarity-driven flow model: Gas and water relative permeability curves. 


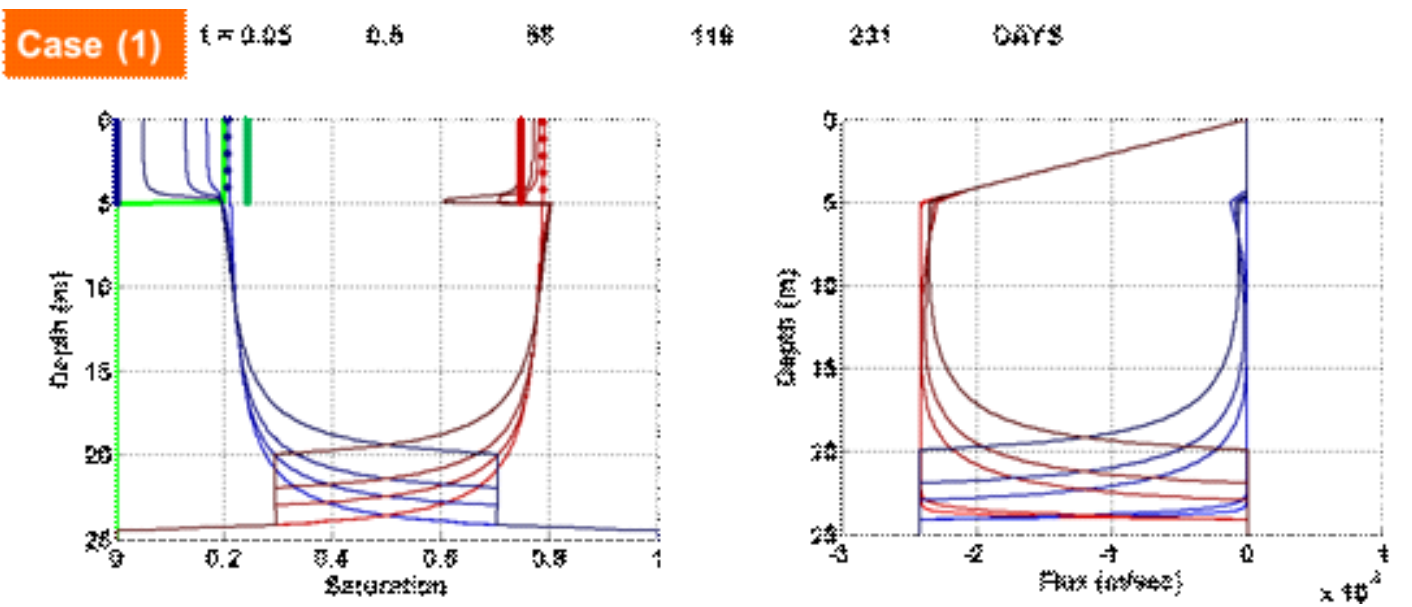

Fig. 43. Model results for Case 1, in which large hydrate growth rate imposed in a $5 \mathrm{~m}$ layer of sediment at the top of a $25 \mathrm{~m}$ thick formation. The lower 20 meters of the formation are below the BGHSZ. (left). Saturation profiles (blue $=$ water, red $=$ gas, green $=$ hydrate) at the times indicated at the top of the image. Darker shades correspond to later times. Initial and final saturations in the $5 \mathrm{~m}$ layer shown as dotted and heavy lines, respectively. (right) Phase fluxes (blue $=$ water, red $=$ gas) in the formation at the times indicated at top of image. Darker shades correspond to later times. Negative values of flux mean upward flow. The flux of water phase is forced to nearly zero between $5 \mathrm{~m}$ and $15 \mathrm{~m}$ by the large flux of gas phase, which has much larger mobility. Thus hydrate formation ends after all the water in the top $5 \mathrm{~m}$ of the sediment is consumed (note final saturations in left panel, indicated by heavy lines in top $5 \mathrm{~m}$ ).

\section{Case 1}

A large rate of hydrate growth $(6 \mathrm{~m} / \mathrm{y})$ is imposed in the example shown in Fig. 43. Large growth rates require large fluxes of gaseous and aqueous phases. These can be provided only by pressure driven flow; the product of saturation gradient and the slope of the $P_{c}-S_{w}$ characteristic curve is simply not large enough to provide large water flux. Thus the large rate of hydrate growth leads to a viscous-dominated fractional flow in which gas flux overwhelms the capacity for water flux. The GWC rises at the speed of the classical Buckley-Leverett shock front (Fig. 43, left panel); above the GWC the saturation profile has a short transition zone then is essentially constant. The flux of aqueous phase is negligible above the GWC (Fig. 43, right panel). Since only gas is arriving at the GHSZ at $5 \mathrm{~m}$ depth, hydrate formation is limited to the water available in the GHSZ. The final state in the GHSZ shows a modest $S_{h}$ of $25 \%$ (heavy green line, Fig. 43 left panel) and gas saturation of 75\% (heavy red line, Fig. 43, left panel), slightly below the initial value of $80 \%$. 

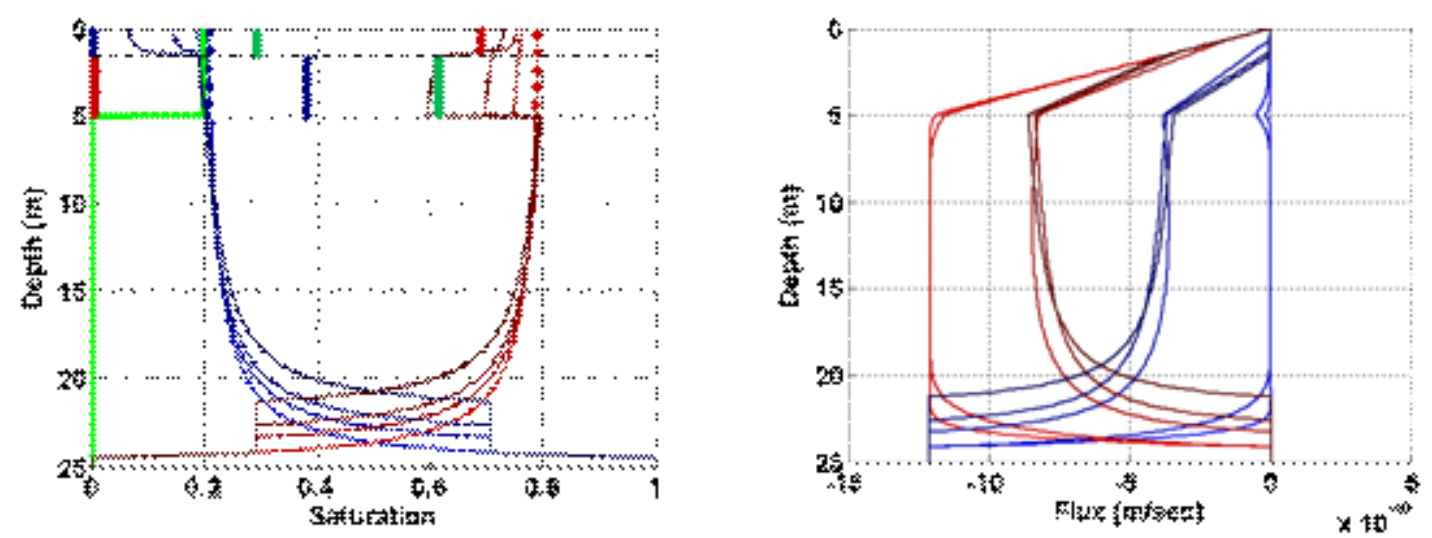

Fig. 44. Model results for Case 2, in which rate of hydrate growth is moderate. (left). Saturation profiles (blue $=$ water, red $=$ gas, green $=$ hydrate). Darker shades correspond to later times. Initial and final saturations in the $5 \mathrm{~m}$ layer shown as dotted and heavy lines, respectively. (right) Phase fluxes (blue = water, red = gas) in the formation at the times indicated at top of image. Darker shades correspond to later times. Negative values of flux mean upward flow. The flux of water phase at early times is essentially zero because the saturation gradient is too small to sustain the rates required by hydrate growth; thus in the top $1.5 \mathrm{~m}$ of the GHSZ, all the water is consumed to form hydrate. At later times the water phase saturation gradient can sustain the needed upward flux of $4 \times 10^{-10} \mathrm{~m} / \mathrm{s}$, while the upward gas phase flux is twice the water flux. The final saturation of hydrate is large (62\%), and the final water saturation is $38 \%$.

\section{Case 2}

Moderate rates of hydrate growth $(0.3 \mathrm{~m} / \mathrm{y})$ require moderate fluxes of water and gas phases (Fig. 44). These can be provided by a combination of pressure-driven and capillarity-driven flow. Though the saturation profiles are similar to Case 1, the flux profiles are quite different, with water flux being about half the gas flux. The water and gas fluxes reach a steady value in the upper part of the domain below the GHSZ; they vary with time in the lower part as the GWC rises. As a result hydrate formation proceeds with all the gas arriving at the GHSZ being converted to hydrate. Thus in contrast to Case 1, the final state in the lower $3 \mathrm{~m}$ of the GHSZ in Case 2 shows a large $S_{h}$ of $62 \%$ and no remaining gas saturation; instead the final water saturation is $38 \%$. The top $2 \mathrm{~m}$ of the GHSZ show a similar final state to Case 1 because during early time the flux of water to the top $2 \mathrm{~m}$ could not be sustained. 


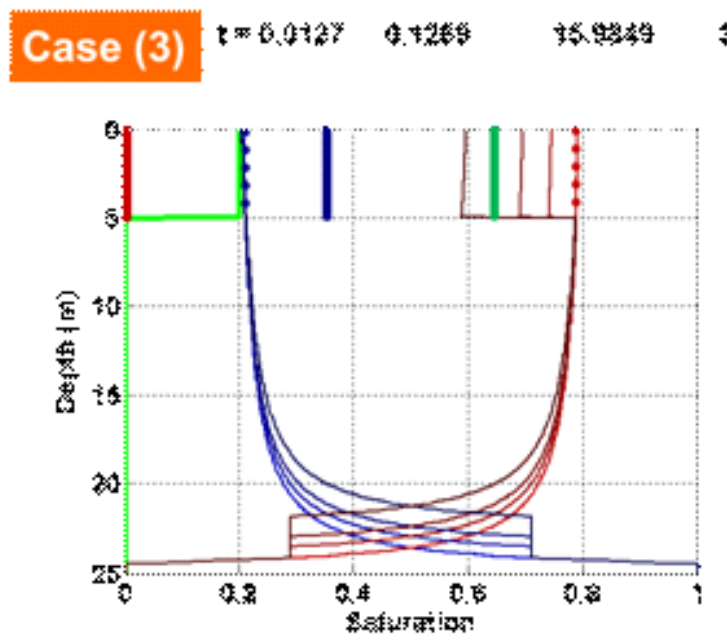

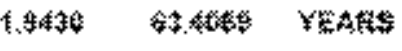

Fig. 45. Model results for Case 3, in which small hydrate growth rate is imposed. (left). Saturation profiles (blue $=$ water, red $=$ gas, green $=$ hydrate) at the times indicated at the top of the image. Darker shades correspond to later times. Initial and final saturations in the $5 \mathrm{~m}$ layer shown as dotted and heavy lines, respectively. (right) Phase fluxes (blue = water, red = gas) in the formation at the times indicated at top of image. Darker shades correspond to later times. Negative values of flux mean upward flow. The flux of water phase quickly approaches a steady value of $1 \times 10^{-10} \mathrm{~m} / \mathrm{s}$, while the upward gas phase flux is $1.4 \times 10^{-10}$ $\mathrm{m} / \mathrm{s}$.

\section{Case 3:}

If the rate of hydrate growth is small $(0.06 \mathrm{~m} / \mathrm{y})$, then the required fluxes of gas and water are proportionately smaller, as seen in Fig. 45 (right panel). In this case, the pressure-driven flow is quite small, and the saturation-gradient driven flow accounts for most of the aqueous phase flux. The systems quickly approaches a pseudo-steady state, in which the GWC rises slowly while the saturation profiles above the GWC stay nearly the same shape (Fig 45, left panel). Thus the fluxes of both phases are nearly constant (Fig 45, right panel). In other words, the small steady rate of hydrate growth imposed on the system leads to small steady capillarity-dominated fluxes of fluids into the GHSZ. These steady phase fluxes determine the value of $R_{v}$, the fraction of total flux which is gas phase, to approach 0.59 at a depth of $5 \mathrm{~m}$, where the fluids enter the GHSZ. The flux of water is almost entirely due to capillarity.

As described in the discussion of volumetric considerations earlier in this report, the value of $R_{v}$ and the initial gas saturation determine the final hydrate saturation and the saturation of the other phase (gas or water). During the times shown here, the water saturation in the GHSZ (top 5 $\mathrm{m}$ of formation) is remaining steady at just over its initial value of $20 \%$, and the hydrate saturation increases as the gas saturation decreases. Thus from Fig. 4 we expect the final $S_{h}$ to be $80 \%$. But in the transport model we find $S_{h}$ is about $65 \%$ (heavy green line in left panel of Fig 45). The difference is due to the relative permeability curves, which show a residual gas saturation of 0.30 (Fig. 42). Thus the pressure-driven transport of gas into the GHSZ stops when $S_{g}$ reaches 0.3 in the GHSZ, and subsequently only water flows into the GHSZ from below. During this period $R_{v}$ is zero, and the remaining gas phase is converted to hydrate. Consequently the final hydrate saturation is smaller than expected from Fig. 4, since Fig. 4 ignores any transport limitations. In effect Fig. 4 assumes zero residual gas saturation, and thus provides an upper bound on the final hydrate saturation. 
We may also place the results of the capillarity-driven transport model for Case 3 in the context of the pore-scale imbibition model developed earlier in this report. The volumetric model depicted in Fig. 4 neglects all considerations of transport mechanism, and a value of $R_{v}=0.59$ in this model yields a final hydrate saturation that corresponds to the initial gas saturation. The pore-scale imbibition model ignores transport at the bed scale. Scenario A of this model, in which water arrives very slowly to the gas/hydrate/water interface, also yields a final hydrate saturation equal to the initial gas saturation, cf. Fig. 14. Scenario A assumed no limitation on the gas phase transport. Thus a common factor in these predictions of large final hydrate saturation is the absence of constraints on gas transport. In contrast the capillarity-driven flux model explicitly accounts for both phase fluxes at the bed scale and therefore must account for the effect of residual gas saturation. It thus yields a smaller final hydrate saturation as discussed above. Similarly, Scenario B of the pore-level imbibition model accounted for the possibility of trapping residual gas, Fig. 15. This yields a smaller final hydrate saturation; compare left and middle panels of Fig. 27, for example. The common factor to the predictions of not-quite-as-large final hydrate saturation is the constraint on gas transport that applies when gas saturation declines to residual.

This analysis shows that the pore-scale imbibition model can be incorporated into the bedscale transport model described in this section via the residual gas saturation. If pore-scale considerations suggest that water reaches the gas/hydrate/water interface in individual pores slowly compared to the rate of hydrate growth at that interface, then the effective residual gas saturation is zero. It is clear that moving the gas relative permeability curve endpoint to $S_{w}=1$ in Fig. 42 would yield a prediction of $S_{h}=80 \%$ in the transport model. The pore-scale imbibition model scenarios show that the characteristic rock properties for the gas/hydrate/water system need not be the same as for the gas/water system. Thus we conclude that the capillarity-driven transport model should in general account for two sets of curves, one set that applies in the GHSZ where three phases (gas, water, hydrate) can co-exist in the pore space, and another conventional set that applies in the not-yet-converted gas reservoir below the GHSZ.
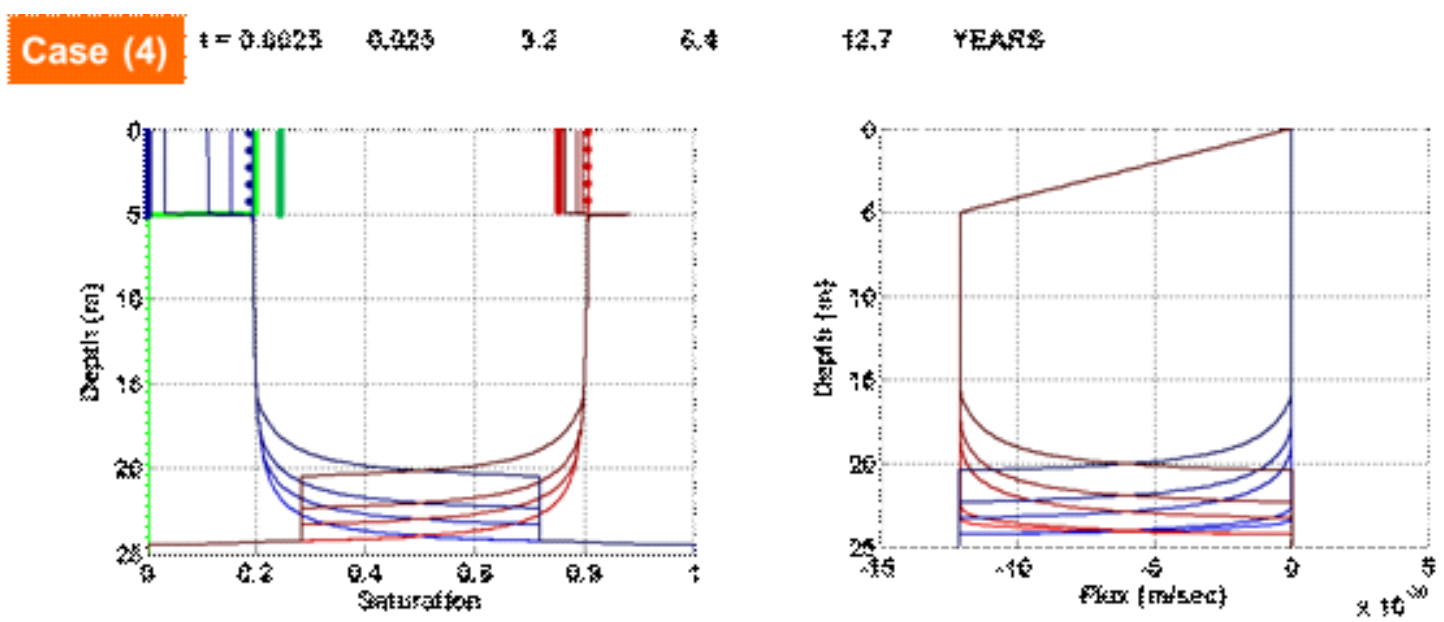

Fig. 46. Model results for Case 2 with a flatter capillary pressure curve. (left). Saturation profiles (blue $=$ water, red $=$ gas, green $=$ hydrate). Darker shades correspond to later times. Initial and final saturations in the $5 \mathrm{~m}$ layer shown as dotted and heavy lines, respectively. (right) Phase fluxes (blue = water, red $=$ gas) in the formation at the times indicated at top of image. Darker shades correspond to later times. Negative values of flux mean upward flow. In contrast to Case 2 (Fig. 44) the flux of water phase is essentially zero because $d P_{c} / d S_{w}$ is smaller. Thus the capillarity-driven flux is too small to sustain the rates required by hydrate growth, and all the water in the GHSZ is consumed to form hydrate. 


\section{Case 4}

To confirm the importance of the contribution of capillarity to the water phase flux, we rerun Case 2 with a shallower capillary pressure curve. This means that the gradient of $P_{c}$ vs $S_{w}$ is smaller. Consequently the capillarity-driven flux is smaller for same saturation gradient. The smaller capillarity-driven flux of water proves insufficient to supply the imposed rate of hydrate growth. Hence the flow in this case reverts to the viscous dominated flow of gaseous and aqueous phases as in Case 1. Therefore, the gas flux becomes dominant and prevents any water reaching the GHSZ. Thus the resulting hydrate saturation is about $25 \%$, and a gas saturation remains in the GHSZ. Comparing Cases 2 and 4 shows that for the same rate of hydrate formation, the final state of the system is determined by the characteristic capillarity and relative permeability curves of the host sediment.

If in nature the rate of hydrate formation is limited by the rate of descent of the BGHSZ, which is in order $10^{-3} \mathrm{~m} / \mathrm{y}$ or less for the relevant cooling period for the Alaska North Slope, then we are in a regime like Case 3 where capillarity driven flux is the primary means of transporting fluid to GHSZ. We conclude that the proportion of gas and water fluxes to the GHSZ (the quantity $R_{v}$ ) is controlled by the relative permeability and capillary pressure characteristics of the domain. For typical capillary and relative permeability characteristics the value of $R_{v}$ converges to values a little less than $R_{v, 1: 1}$ and remains at this value until $S_{g}$ reaches $S_{g r}$ at which point $R_{v}$ goes to zero. Thus we get $S_{h}$ not quite as large as $S_{g, i}$ in the top of the gas reservoir. The rest of the profile can therefore be estimated with the same average value of $R_{v}$, using the simpler model that accounts only for volumetric changes and sedimentology, described in previous section of this report.

\section{Summary of Applicability of Capillarity-Driven Transport Model for Conversion of Gas Reservoirs to Hydrate}

We conclude that capillarity-driven transport to the GHSZ from below is the key mechanism of fluid transport when a gas reservoir is converted to hydrate by a descending BGHSZ. The model computes fluxes due to buoyancy, pressure gradient and saturation gradient in response to the volume changes associated with hydrate formation. The model predicts that capillarity-driven flux is dominant mode of water transport and that this flux is sufficient to supply the needs of relatively slow rates of hydrate formation. The model yields an a priori value of $R_{v}$, the volumetric parameter (fraction of total fluid phases movement made up by gas) which determines the final hydrate saturation, given the initial gas saturation. For typical multiphase flow characteristics (relative permeability and capillary pressure) the predicted $R_{v}$ gives a large hydrate saturation close to those observed in Mt. Elbert and Mallik wells.

Only if the rate of descent of BGHSZ is large (and hence the rate of hydrate generation is large) is the rate of water flux too small to supply the need. In this case the model predicts smaller hydrate saturations and large gas saturation. Since gas saturations are not observed in the GHSZ at Mt. Elbert and Mallik wells, this suggests that the rate of fluid transport may control the rate of hydrate formation if the BGHSZ descends rapidly. This is consistent with the experimental results presented in the next section, in which the BGHSZ was moved $1 \mathrm{~m}$ in 15 days or about $25 \mathrm{~m} / \mathrm{y}$. Hydrate formation occurred at a steady rate that was considerably lagged the rate of cooling.

These considerations suggest that in nature, fluxes of gas and water from below the GHSZ establish saturation profiles that balance the gradients due to buoyancy, pressure and capillarity. At any given location of the BGHSZ, the balance leads to a characteristic value of $R_{v}$, the 
fraction of fluid phase transport made up of gas. This value combined with the presumed value of residual gas saturation in the GHSZ leads to hydrate saturations less than or equal to the initial gas saturation at that location, depending on the magnitude of residual saturation. This is the reason that the simpler bed-scale model (presented in earlier sections of this report, and which accounts for volumetric changes and sedimentological variation for a user-prescribed value of $R_{v}$ ) correctly predicts the Mt. Elbert well saturation profile when the appropriate value of $R_{v}$ is given.

\section{Gas Reservoir Conversion to Hydrate: Experimental Validation of Fluid Transport in Response to Hydrate Formation}

\section{Descending Bottom of Methane Gas Hydrate Stability Zone Experiment Description}

Overview

In collaboration with Dr. Timothy Kneafsey of Lawrence Berkeley National Laboratory, an experiment was conducted in which the base of gas hydrate stability was slowly moved down a sandpack containing gas and water. The apparatus was built in Dr. Kneafsey's lab at LBNL, and the experiment was conducted there in the summer of 2011.

A pressure vessel was packed with alternating 'fine' and 'coarse' fractions of silica sand (US Silica F-110, 120 $\mu \mathrm{m}$ mean diameter), which were previously separated using a mechanical sand shaker. The pressure vessel was saturated with de-ionized water, filling the pore space. A vacuum was drawn on the reservoir before it was pressurized with methane to $\sim 800 \mathrm{psig}$. To establish initial gas saturation in the sand pack, a valve on the bottom of the pressure vessel was opened and water was allowed to flow into the reservoir, replaced by methane gas at the top and mid point of the sand pack. About $360 \mathrm{~g}$ of water was present in the reservoir at the end of the water displacement. Thus the corresponding volume of methane, $\sim 360 \mathrm{~mL}$, had been distributed in the sand pack. Porosity of the sandpack is $\approx 36 \%$. The vessel cooling jackets were progressively switched from $10^{\circ} \mathrm{C}$ to $1.5^{\circ} \mathrm{C}$ coolant from top to bottom, bringing a larger top portion of the sand pack into the hydrate stability zone each time. A cooling jacket was only switched over once minimal incremental hydrate formation was taking place.

A summary of different steps of the apparatus setup is as follows:

- 3"' diameter, 40"' long, 4554mL vessel dry packed with alternating layers of 'fine' and 'coarse' sand, leaving $1633 \mathrm{~mL}$ pore volume $\rightarrow$ Porosity: $\emptyset \approx 36 \%$.

- Vessel vacuum saturated with de-ionized water (1633mL of water added to system)

- $3707 \mathrm{~mL}$ reservoir evacuated, then pressurized with methane $(3707 \mathrm{~mL}$ of methane at $18^{\circ} \mathrm{C}$ and 800 psig added to system)

- System closed while $300 \mathrm{~mL}$ of water drained and pumped from vessel to reservoir, leaving $300 \mathrm{~mL}$ of gas in vessel

- Vessel uniformly cooled to $10^{\circ} \mathrm{C}$, then each cooling loop changed to $1.5^{\circ} \mathrm{C}$ coolant from top to bottom progressively

\section{Grain Size Distribution}

Silica sand (US Silica F-110, 120 $\mu$ m mean diameter) was separated into a 'fine' fraction and a 'coarse' fraction using a mechanical sand shaker. To measure the grain size distribution of each fraction, three $\sim 100 \mathrm{~g}$ samples of each fraction were each further separated by grain size. 
Two different stacks of six progressively smaller sieves were used in a mechanical sand shaker. The results of these measurements are shown in Figs. 47 and 48.

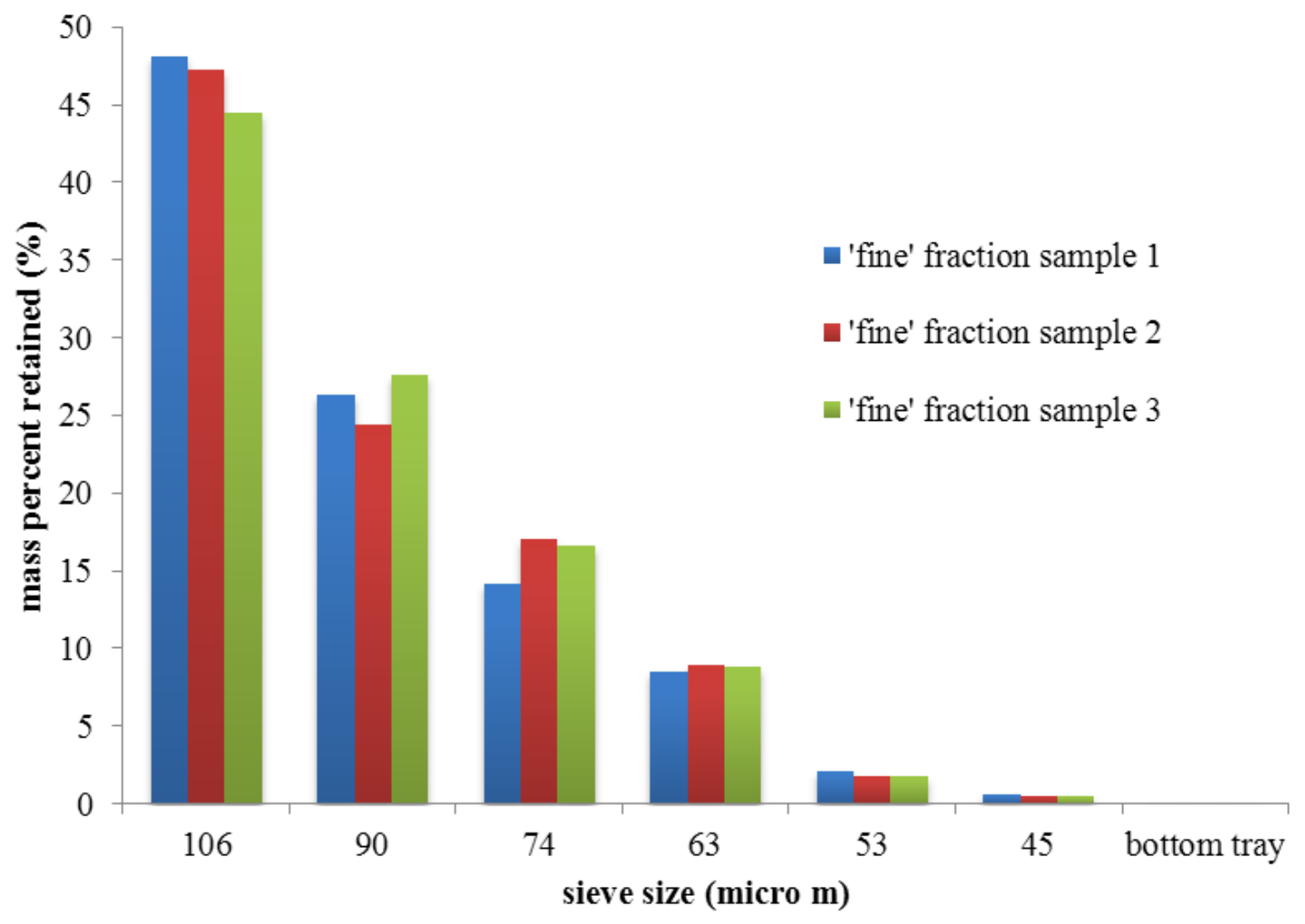

Fig. 47. Fine fraction mass percent sand retained versus sieve size. 


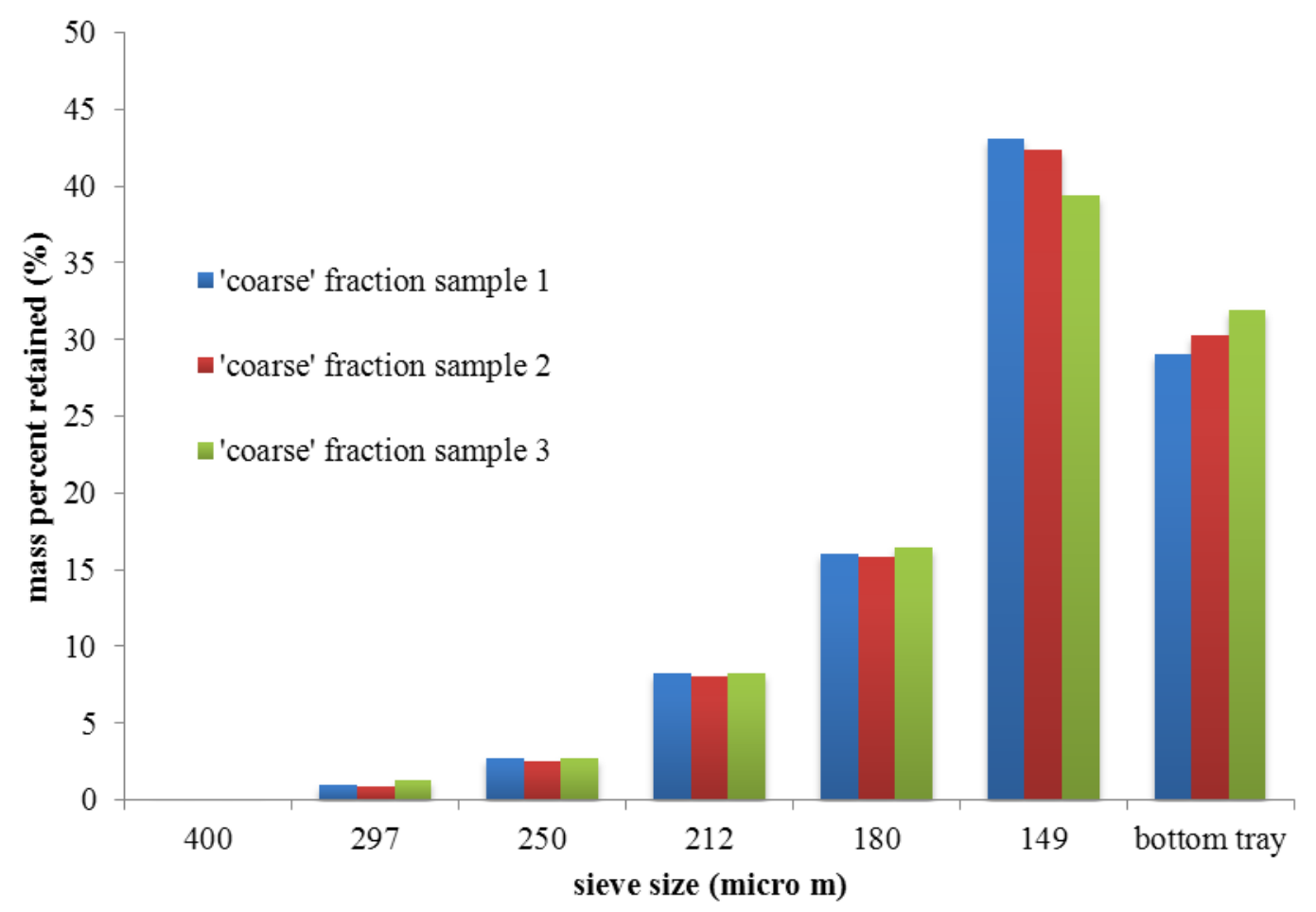

Fig. 48. Coarse fraction mass percent retained versus sieve size.

\section{Drainage Capillary Pressure Curve}

To measure the drainage capillary pressure versus water saturation curve of each fraction, three $\sim 60 \mathrm{~g}$ samples of each fraction were tested using the 'porous plate method'. The samples were placed in cups with porous ceramic bases (soil retainer assemblies, part number 0676, Soilmoisture Equipment Corp.). The samples were then vacuum saturated with de-ionized water, weighed and placed in a vessel fitted with a porous plate ( 5 bar pressure plate extractor, part number 1600, Soilmoisture Equipment Corp.). See Fig. 49. 


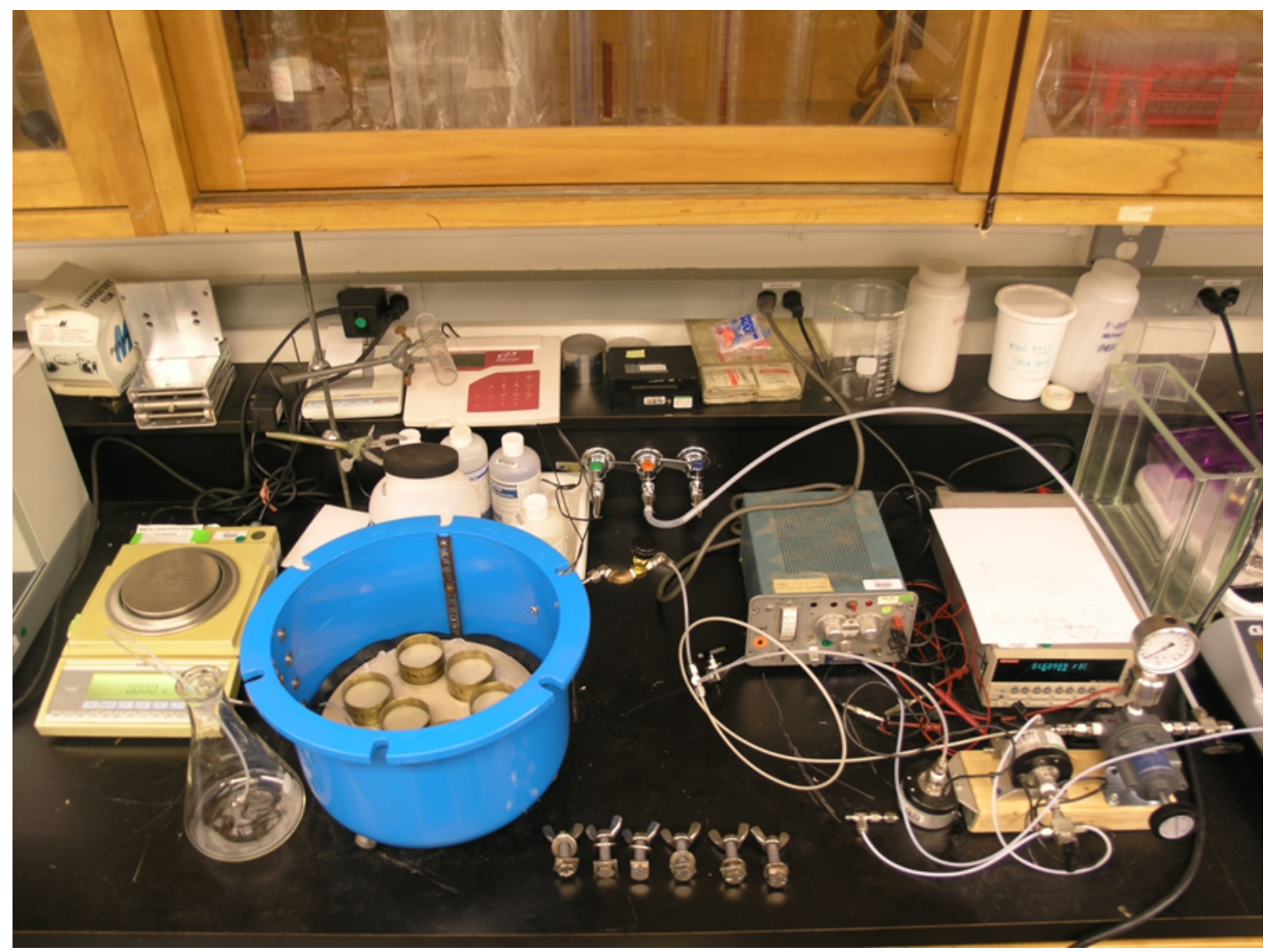

Fig. 49. The porous plate capillary pressure vessel and associated equipment in the laboratory of Dr. Tim Kneafsey at Lawrence Berkeley National Laboratory.

The vessel was closed and pressurized with laboratory air at controlled pressure via a forward pressure regulator. Displaced de-ionized water accumulated in a flask. The system was left at each pressure for sufficient time to reach capillary equilibrium, determined by observation of no further water displacement from the vessel. At this time the vessel was opened and each sample cup weighed. The cups were then returned to the vessel and the air pressure increased by $\sim 0.5$ psi. This process was repeated up to a pressure of 3 psi.

The results of these measurements are shown in Fig50. 


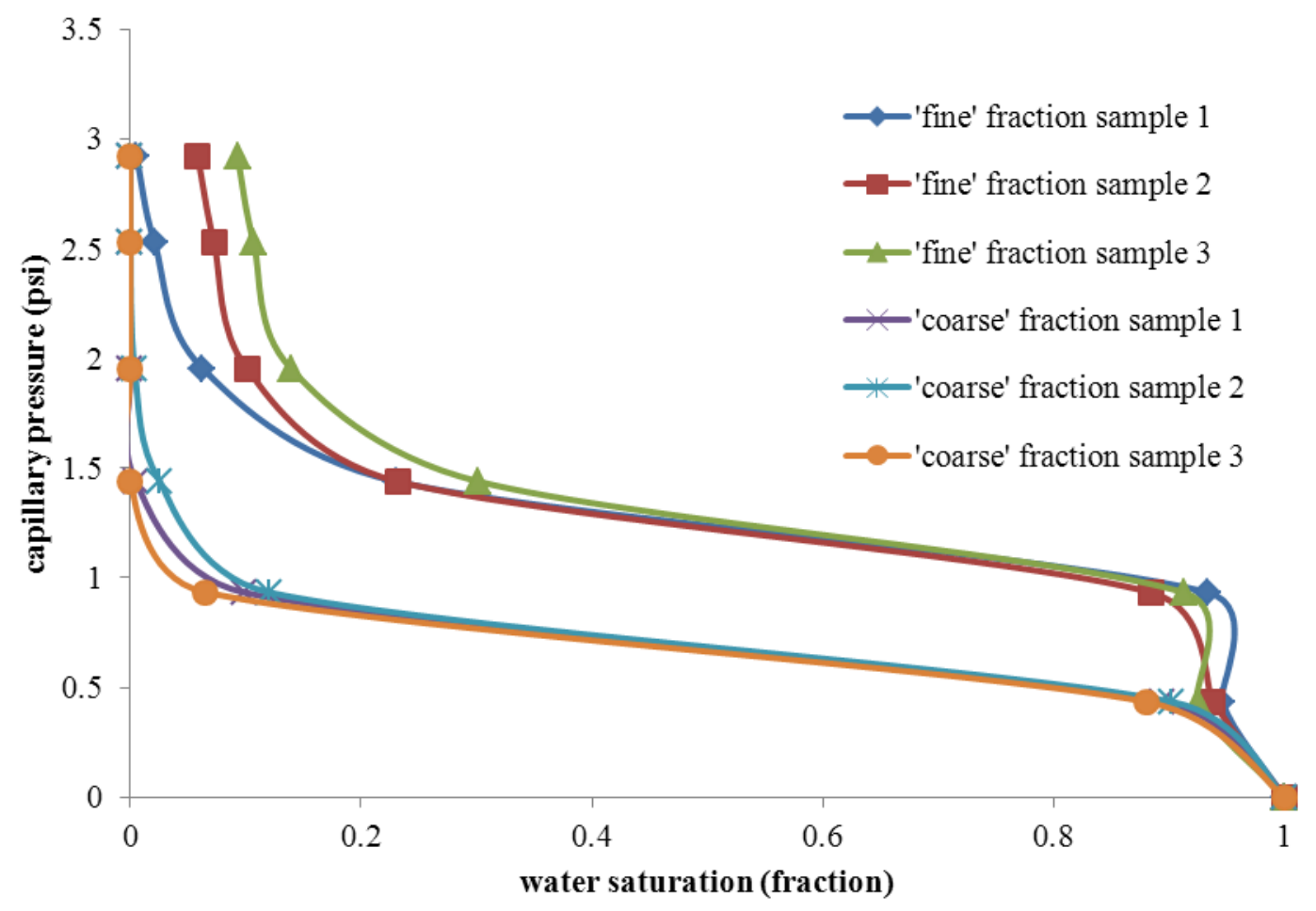

Fig. 50. Capillary pressure versus water saturation for fine and coarse fractions of sand.

\section{Experimental Hardware Description}

Two 'thermocouple strings' were constructed to allow the measurement of temperature at multiple points along their length. Each 'thermocouple string' was a 3/8 inch OD aluminum tube, fitted with ten type $\mathrm{T}$ thermocouples that were each spaced 4 inches apart, filled with epoxy. The two thermocouple strings were fitted to an end cap of a 3 inch diameter $\mathrm{x} 40$ inch long aluminum pressure vessel, one in the center and one offset from center. These twenty thermocouples were labeled T1 to T10, top to bottom, for the sensors in the center mounted thermocouple string and T11 to T20, top to bottom, for the sensors mounted in the outer thermocouple string. Two pressure transducers were fitted to the vessel, one at each end and labeled upper and lower.

The pressure vessel was mounted vertically. Ten discrete cooling jackets were constructed from multiple wraps of soft rubber tubing. These were labeled 1 to 10 from top to bottom. The vessel and cooling jackets were insulated with closed cell foam. Two chillers were filled with ethylene glycol. One chiller was set at $10{ }^{\circ} \mathrm{C}$ (a temperature outside the hydrate stability zone) and the other set at $1.5{ }^{\circ} \mathrm{C}$ (a temperature inside the hydrate stability zone for the range of pressures expected). The chillers were connected to the ten cooling loops via insulated tubing, manifolds, valves and $\mathrm{Y}$ connectors, allowing the coolant temperature circulated through each cooling jacket to be selected independently. A thermocouple was placed in the ethylene glycol tank of the chiller set to $1.5^{\circ} \mathrm{C}$ and labeled chiller temperature.

A one-gallon pressure vessel, termed the 'reservoir' (part number 304L-HDF4-1GAL, Swagelok) was fitted to a stand and set at an angle of $\sim 45$ degrees from horizontal. The vessel was placed on a balance (Sartorius). A thermocouple was placed on the reservoir and labeled reservoir temperature.

The top of the reservoir was connected to the top of the pressure vessel and a point halfway between the top and bottom of the pressure vessel. The bottom of the reservoir was connected to 
the bottom of the pressure vessel. Flexible PEEK pressure lines were used for the connections to the reservoir to minimize external forces applied, allowing the mass of the reservoir and fluids to be accurately recorded by the balance. A schematic and photo of the experimental setup are shown in Figs. 51 and 52.

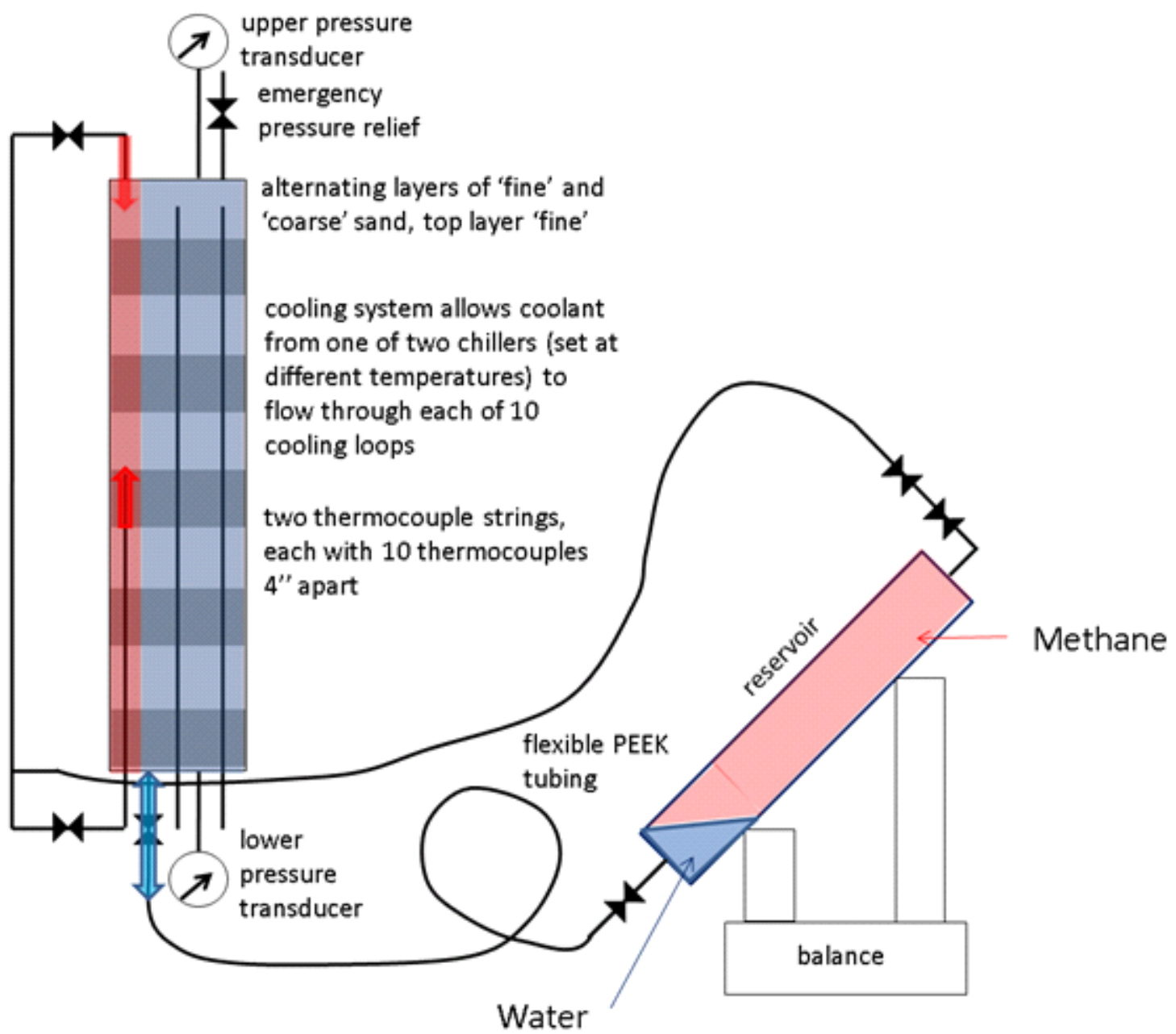

Fig. 51. Schematic of the descending BGHSZ experiment. 


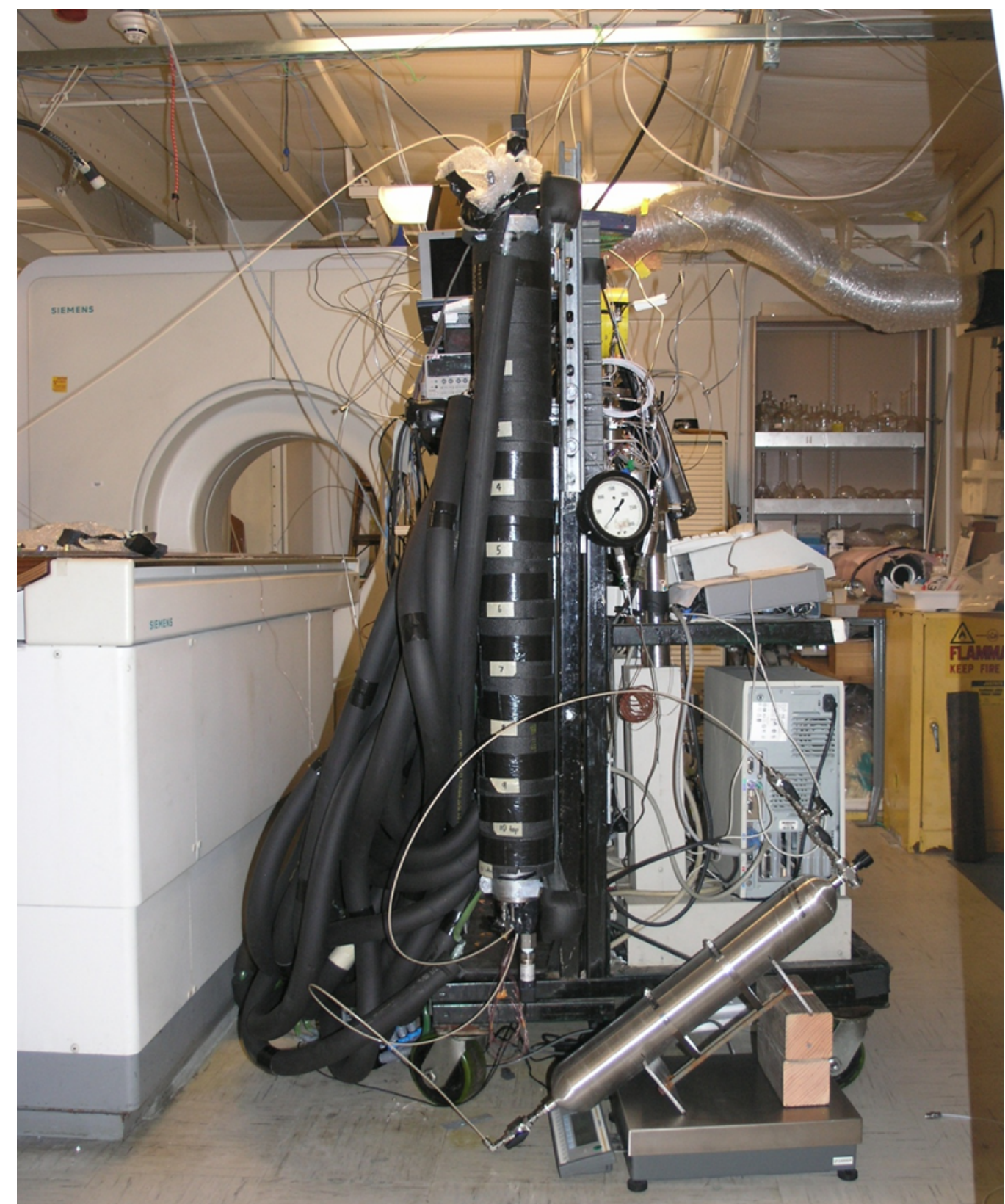

Fig. 52. Descending BGHSZ experimental setup in the laboratory of Dr. Tim Kneafsey at Lawrence Berkeley National Laboratory.

The twenty-two thermocouples and two pressure transducers were logged using a Keithley data logger writing to an Excel spreadsheet. The balance was logged using a software wedge writing to a text file.

\section{Setting up the Sand Pack, Water and Methane}

The vessel was packed with alternating fractions of coarse and fine fractions of silica sand. Each fraction was poured into the pressure vessel in small stages and tamped with a plastic rod. The height and mass of each fraction of sand is shown in Table 3. 
Table 3. Height and Mass of Each Alternating Sand

Fraction

\begin{tabular}{cccc}
\hline & Fraction & Height (in) & Mass (g) \\
\hline Top & fine & 4.6 & 641.8 \\
& coarse & 3.9 & 741.0 \\
& fine & 4.0 & 751.8 \\
& coarse & 3.4 & 670.0 \\
& fine & 3.9 & 706.5 \\
& coarse & 3.3 & 734.3 \\
& fine & 4.5 & 795.3 \\
& coarse & 4.0 & 751.5 \\
Bottom & fine & 4.5 & 883.2 \\
& coarse & 3.9 & 1057.6 \\
\hline
\end{tabular}

The pressure vessel internal volume minus that occupied by internals was $4551.1 \mathrm{~mL}$. The volume of sand packed into the vessel was $2918.1 \mathrm{~mL}$, calculated from the total mass and density $\left(\rho_{\text {sand }}=2.65 \mathrm{~g} / \mathrm{mL}\right)$. The difference was $1633.0 \mathrm{~mL}$ of pore volume or an average of $35.9 \%$ porosity.

The sand filled pressure vessel was evacuated with a vacuum pump and then scanned with a medical computed tomography (CT) scanner. The pressure vessel was then saturated with deionized water, filling the pore space with 90.64 moles of water $\left(\rho_{\text {water }}=1.00 \mathrm{~g} / \mathrm{mL}\right)$. The sand and water filled pressure vessel was then re-scanned with the CT scanner. A vacuum was drawn on the reservoir before it was pressurized with methane to $\sim 800$ psig.

Methane density data versus temperature and pressure was obtained from the NIST Chemistry WebBook, which uses a custom equation of state for methane (Setzman and Wagner, 1991). Methane density was calculated across the temperature and pressure range of interest for the experiment at isotherms from $0{ }^{\circ} \mathrm{C}$ to $22{ }^{\circ} \mathrm{C}$ in one-degree increments and across a pressure range of 644.7 psia to 854.7 psia. Each isotherm (density versus pressure) was fitted with a linear trend-line. The slope and intercept of each trend-line was plotted versus temperature and quadratic functions fitted. This allowed a simple equation of state proxy for methane to be formulated, one that matches the NIST data to $0.2 \%$ for density. Using the equation of state proxy described, the reservoir temperature and upper pressure transducer pressure, along with the reservoir volume of $3706.8 \mathrm{~mL}, 9.53$ moles of methane was supplied to the system during pressurization.

The gas phase was assumed to be pure methane, since at such low temperatures the water vapor pressure contributes a very small mole fraction of water to the vapor phase. The solubility of methane in water is very small and as such methane dissolution into the aqueous phase is neglected.

\section{Experimental Procedure}

To establish initial gas saturation in the sand pack, a valve on the bottom of the pressure vessel was opened and water was allowed to flow into the reservoir, replaced by methane gas at the top and mid point of the sand pack. Once the indicated mass on the balance reached close to a steady state due to capillary equilibrium, an ISCO syringe pump was used to withdraw small (20 $\mathrm{mL}$ and $50 \mathrm{~mL}$ ) volumes of methane from the reservoir and inject them into the top of the sand pack, leading to further displacement of water into the reservoir. After a small number of cycles of methane reinjection, no significant change in mass indicated on the balance was observed, 
indicating methane breakthrough. At this time hydraulic connectivity was re-established between the bottom of the reservoir and the bottom of the pressure vessel.

$359.7 \mathrm{~g}$ of water was present in the reservoir at the end of the gravity and gas injection driven water displacement, with the balance remaining in the sand pack. Thus the same volume of methane, $359.7 \mathrm{~mL}$, had been distributed in the sand pack.

All pressure vessel cooling jackets were set to the $10{ }^{\circ} \mathrm{C}$ chiller and the sand pack cooled from room temperature. After reaching a steady state of $10{ }^{\circ} \mathrm{C}$, the pressure vessel cooling jackets were progressively switched from $10^{\circ} \mathrm{C}$ to $1.5^{\circ} \mathrm{C}$ coolant from top to bottom, bringing a larger top portion of the sand pack into the hydrate stability zone each time. A cooling jacket was only switched over once minimal incremental hydrate formation was taking place.

At one point the pressure had dropped sufficiently that the system was re-pressurized to ensure adequate methane availability for hydrate formation.

The temperatures measured on the outer thermocouple string are shown in Fig. 53. From $359980 \mathrm{~s}$, cooling loop 2 has been switched to $1.5^{\circ} \mathrm{C}$ coolant. Cooling loop was already circulating $1.5{ }^{\circ} \mathrm{C}$ coolant at that time. From $392600 \mathrm{~s}$ two distinct spikes in temperature are seen, as a result of heat produced during rapid hydrate formation.

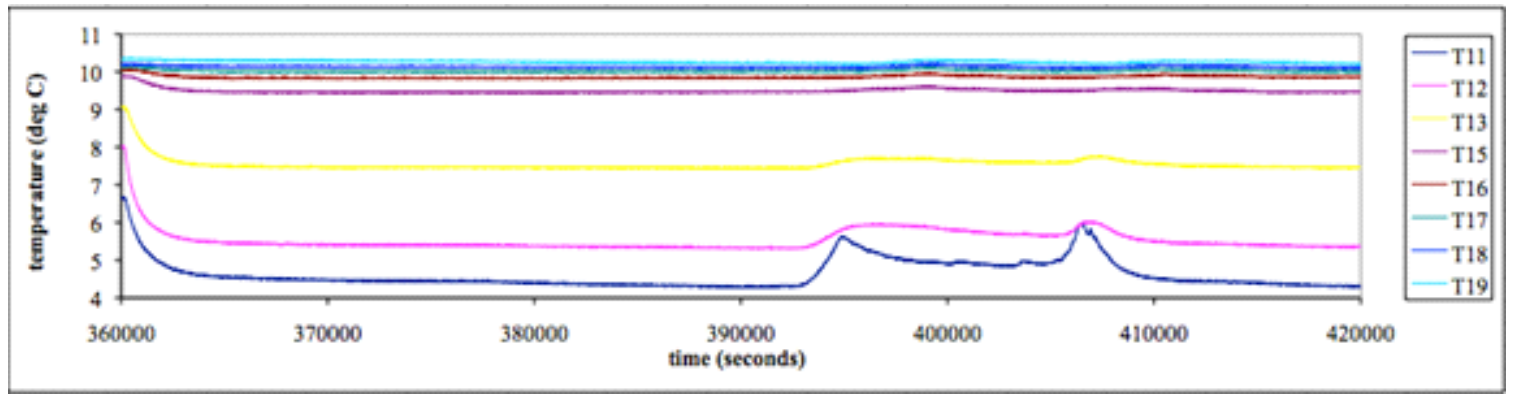

Fig. 53. Outer thermocouple string temperatures versus time.

The variations in reservoir temperature, driven by the ambient conditions prevailing in the room, are shown in Fig. 54.

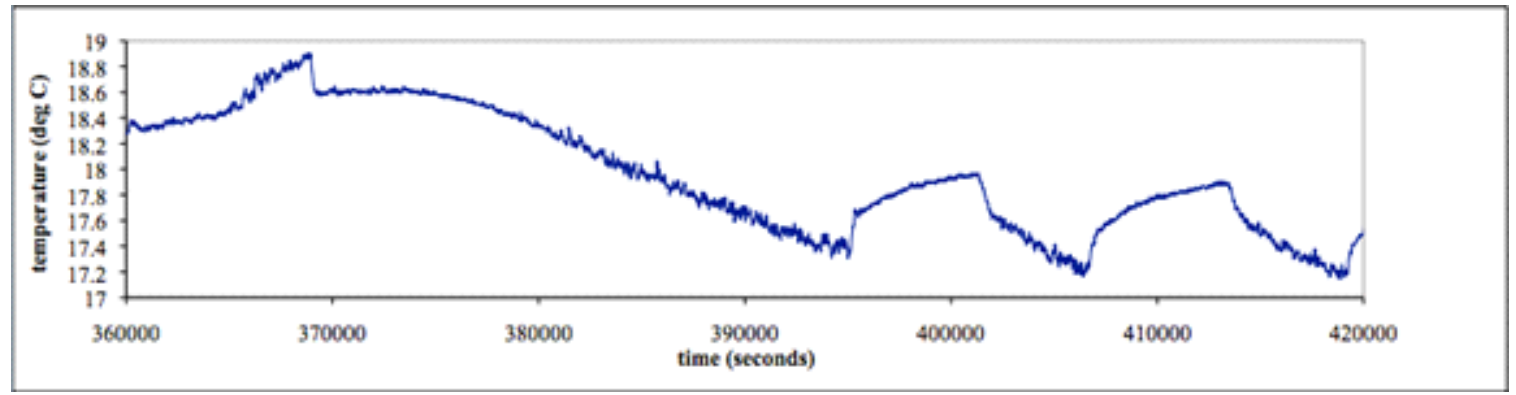

Fig. 54. Room temperature versus time.

The system pressure drop associated with the formation of gas hydrate is shown in Fig. 55. 


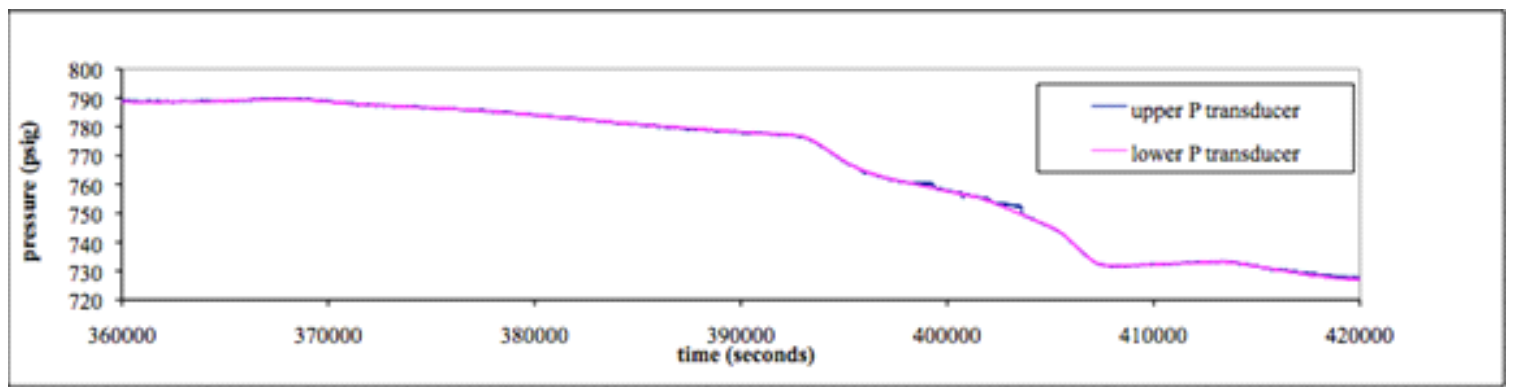

Fig. 55. Pressure versus time.

The indicated mass on the balance is shown in Fig. 56.

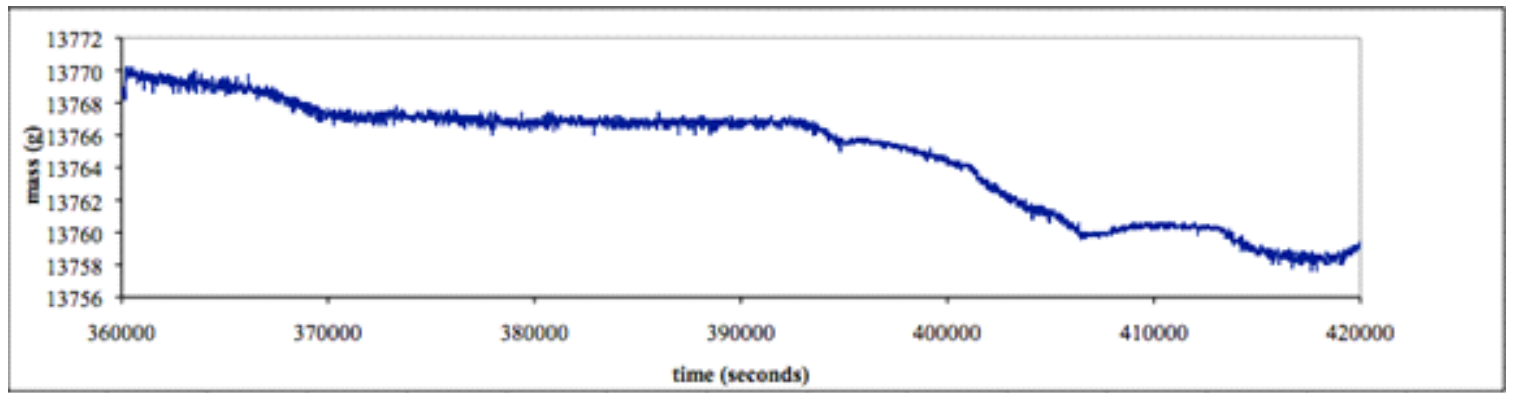

Fig. 56. Indicated mass on balance versus time.

Once the entire sand pack had been brought into the hydrate stability zone, the pressure vessel was scanned once more with the CT scanner, as shown in Fig. 57. 


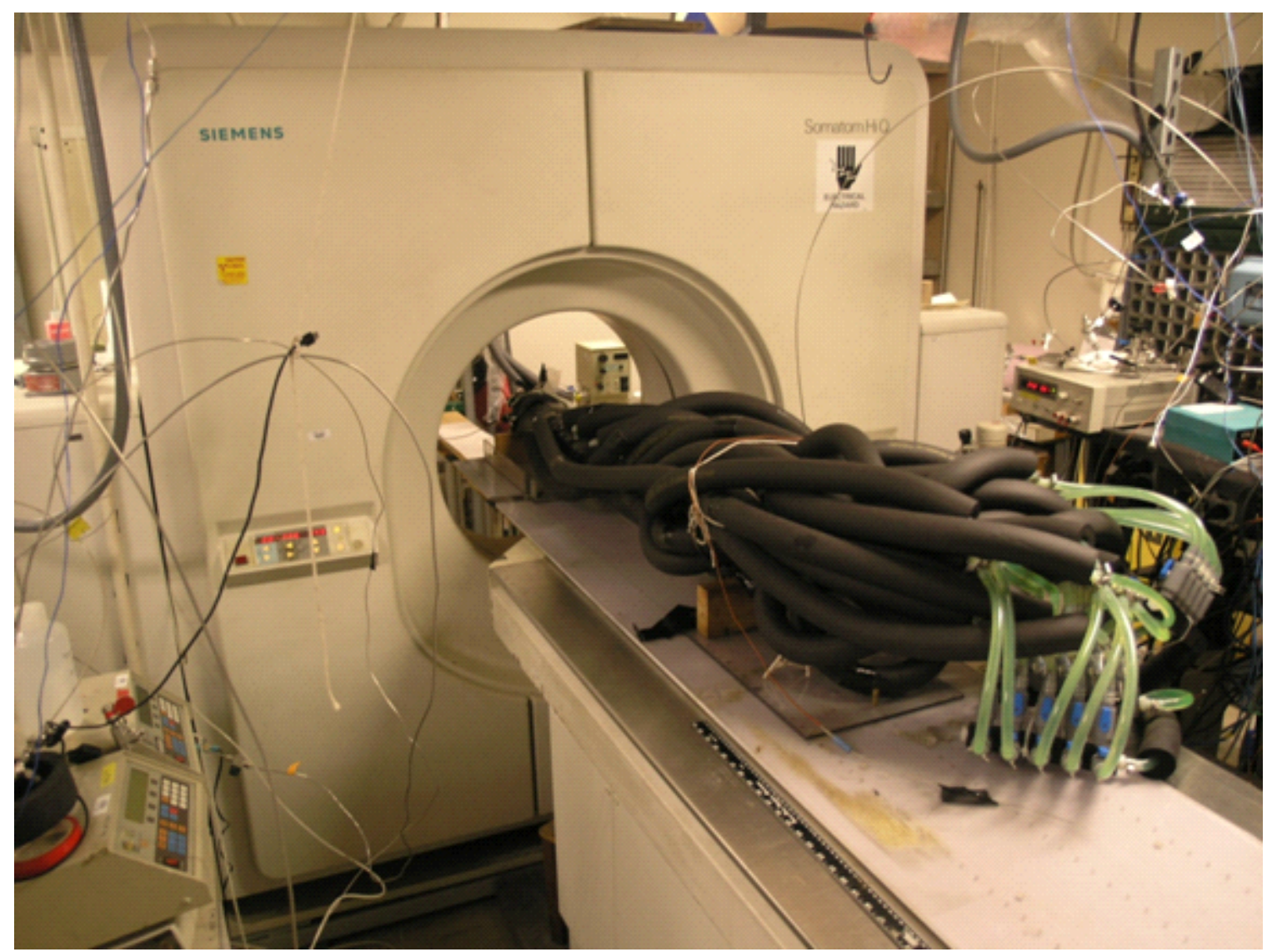

Fig. 57. Scanning pressure vessel, sand pack and hydrate with medical CT scanner in the laboratory of Dr. Tim Kneafsey at Lawrence Berkeley National Laboratory.

\section{Calculations from Raw Data}

At any time during the experiment, the mass indicated on the balance is a sum of the following:

$m_{\text {balance }}=m_{\text {reservoir }}+m_{\text {waterreservoir }}+m_{\text {methanereservoir }}+m_{\text {verticalline }}$

where:

- $\quad m_{\text {balance }}=$ mass indicated on balance $(\mathrm{g})$

- $\quad m_{\text {reservoir }}=$ mass of reservoir $(\mathrm{g})$

- $m_{\text {waterreservoir }}=$ mass of water in reservoir $(\mathrm{g})$

- $m_{\text {methanereservoir }}=$ mass of methane in reservoir $(\mathrm{g})$

- $m_{\text {vertical line }}=$ vertical component of force applied to reservoir, as apparent mass $(\mathrm{g})$

From the volume of the reservoir, the prevailing gas density from the equation of state proxy detailed above and assuming a water density, $\rho_{\text {water }}=1.00 \mathrm{~g} / \mathrm{mL}$, an expression for the mass of water in the reservoir at any time during the experiment can be arranged:

$m_{\text {waterreservoir }}=\frac{m_{\text {balance }}-m_{\text {reservoir }}-m_{\text {verticalline }}-3706.8 * \rho_{\text {methane }}}{1-\rho_{\text {methane }}}$ 
The results from Eq. 90 are shown in Fig. 58.

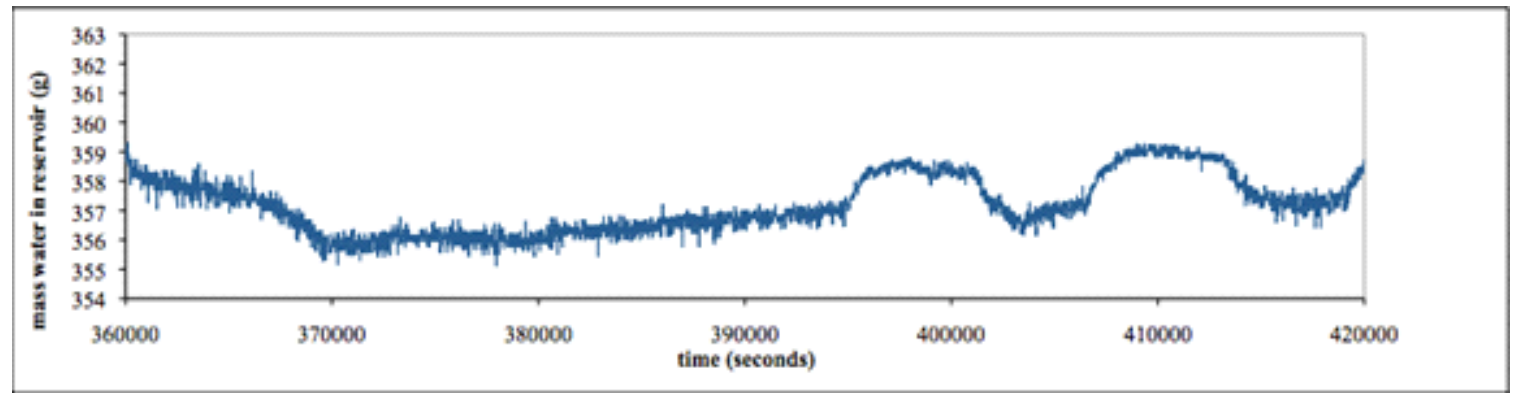

Fig. 58. Mass of water in reservoir versus time.

To approximate the quantity of hydrate formed at any time during the experiment, it was assumed that hydrate occupies the same volume as the water consumed during its formation. This assumption leads to the volume occupied by methane gas remaining unchanged in the presence of hydrate formation. The equation of state proxy was used for gas density, along with the gas volume, $3706.8 \mathrm{~mL}$, to calculate the moles of methane present in gas phase and by difference to the total moles of methane in the system, that present in hydrate. The results of this calculation are shown in Fig. 59.

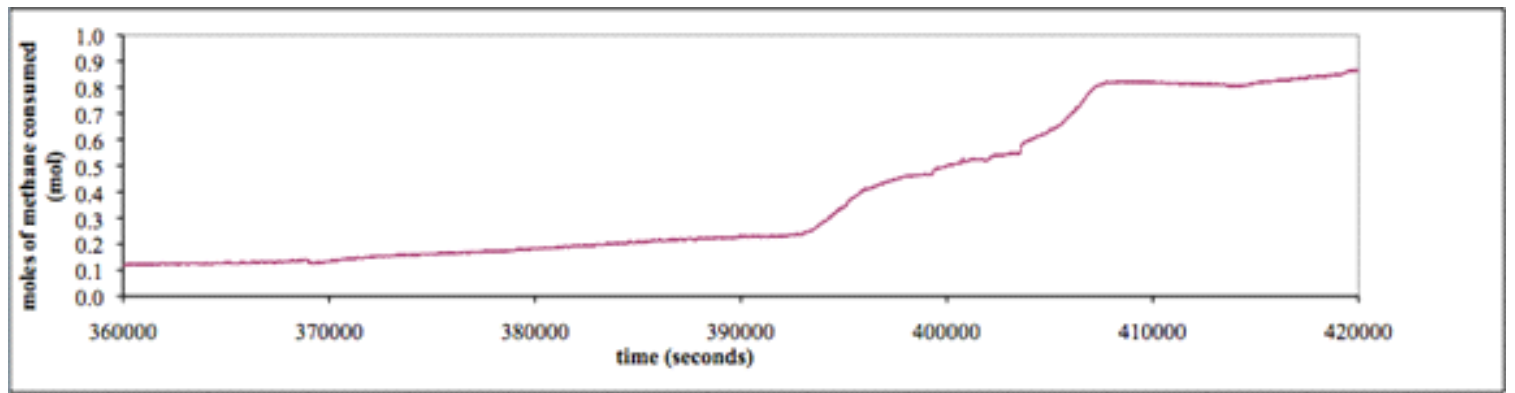

Fig. 59. Methane consumed versus time.

The location of the BGHSZ at the end of each period of cooling was determined from a linear interpolation of the location of the equilibrium temperature read by the outer thermocouple string, at the prevailing pressure. The results are presented in Table 4. 
Table 4. BGHSZ Location and Quantity of Hydrate Formed After Each Period of Cooling

\begin{tabular}{ccccc}
\hline $\begin{array}{c}\text { Bottom Cooling } \\
\text { Loop Circulating } \\
1.5^{\circ} \text { C Coolant }\end{array}$ & Time (s) & $\begin{array}{c}\text { Distance from Top } \\
\text { of Sediment Column } \\
\text { to BGHSZ (in) }\end{array}$ & $\begin{array}{c}\text { Cumulative Moles of } \\
\text { Hydrate Formed at End } \\
\text { of Time Period (mole) }\end{array}$ & $\begin{array}{c}\text { Cumulative } \\
\text { Volume of Hydrate } \\
\text { Formed }\left(\mathrm{cm}^{3}\right)\end{array}$ \\
\hline 1 & $351940-359980$ & 3.7 & 0.07 & 9.7 \\
2 & $359980-446260$ & 8.1 & 0.85 & 113.7 \\
3 & $446260-529080$ & 11.8 & 1.73 & 232.6 \\
3 & $529080-544080$ & 15.2 & 1.80 & 241.2 \\
4 & $544080-705240$ & 17.4 & 2.46 & 330.4 \\
5 & $705240-783060$ & 20.8 & 3.03 & 406.4 \\
6 & $783060-862840$ & 24.5 & 3.18 & 452.2 \\
7 & $862840-943720$ & 28.0 & 3.38 & 472.6 \\
8 & $943720-982960$ & 32.3 & 3.52 & 483.5 \\
9 & $982960-1029780$ & 36.0 & 3.60 & 502.2 \\
\hline 10 & $1029780-1114100$ & 39.9 (entire column) & 3.74 & \\
\hline
\end{tabular}

Note: the system was re-pressurized from $529080 \mathrm{~s}$. The entries at $529080 \mathrm{~s}$ show the location of the BGHSZ and moles of hydrate formed just prior to re-pressurization.

At select times of interest, an accurate calculation of the quantity of hydrate formed was completed. This was done by again using the equation of state proxy for methane, though applied to two separate volumes of gas phase methane. At the temperature indicated by the reservoir thermocouple and the pressure indicated by the upper pressure transducer, one volume of methane occupies the volume of the reservoir not occupied by the volume of the water in the reservoir.

$n_{\text {methane }}=\frac{\left(3706.8-\frac{m_{\text {water }}}{\rho_{\text {water }}}\right)}{v_{\text {methane }}}$

where

- $\quad n_{\text {methane }}=$ number of moles of methane in reservoir (mol)

- $\quad v_{\text {methane }}=$ molar volume of methane $(\mathrm{mL} / \mathrm{mol})$

The remainder of the methane is present in the sand pack in gas phase below the BGHSZ or in hydrate phase within the GHSZ. A fractional term apportioning methane between the two phases was solved for, such that the volume of liquid water plus gas phase methane plus gas hydrate was equal to the total pore volume.

pore volume $=V_{\text {watersandpack }}+V_{\text {methanesanpack }}+V_{\text {hydrate }}$

where

- $\quad$ pore volume $=$ pore volume $(\mathrm{mL})$

- $\quad V_{\text {watersandpack }}=$ volume of water in sand pack $(\mathrm{mL})$

- $V_{\text {methanesandpack }}=$ volume of methane in gas phase $(\mathrm{mL})$

- $\quad V_{\text {hydrate }}=$ volume of hydrate $(\mathrm{mL})$

A hydration number of 6 and hydrate density of $0.925 \mathrm{~g} / \mathrm{mL}$ was assumed. The quantity of methane hydrate formed at the end of each time period calculated by this approach is included in Table 4. This method is more accurate than the approximate method used to produce Fig. 59. However, when comparing the two calculation techniques for all but the first calculation, a 
maximum percentage difference in the number of moles of hydrate calculated is $5 \%$, which is relatively small. The first calculation is based on such a small change in pressure that other effects may dominate the results calculated.

\section{Results-Distribution of Density at End of Experiment}

Figure 60 shows the results of preliminary processing of the CT images acquired in the water-saturated sandpack prior to gas invasion (left panel of Fig. 60) and after the experiment ended, i.e. when the entire column had been cooled to be in the GHSZ and no more water remained in the reservoir (right panel of Fig. 60). The left panel reveals minor variations in packing density within the column. The right panel shows two main regions of relatively low density material (cold colors) in the two coarse-sand layers in the top half of the column. Smaller amounts of low-density material are present in the two uppermost fine-sand layers (orange colors). The preliminary image processing cannot unequivocally attribute the low density regions to hydrate rather than gas. However it is clear that hydrate formed in the experiment, and if that hydrate were concentrated in the upper coarse-sand layers then the CT image would look very much like the right panel in Fig. 60. Thus for the purpose of analyzing this experiment, we will assume that regions of maximum density (yellow) are sand completely saturated with water, while regions of less than maximum density contain hydrate and water. 


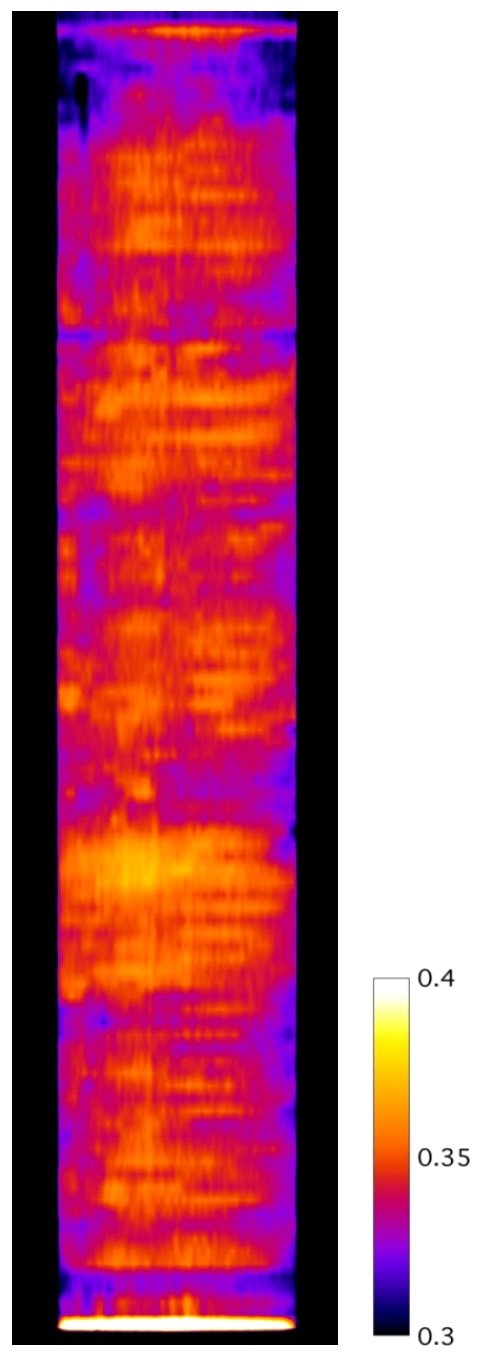

(a)

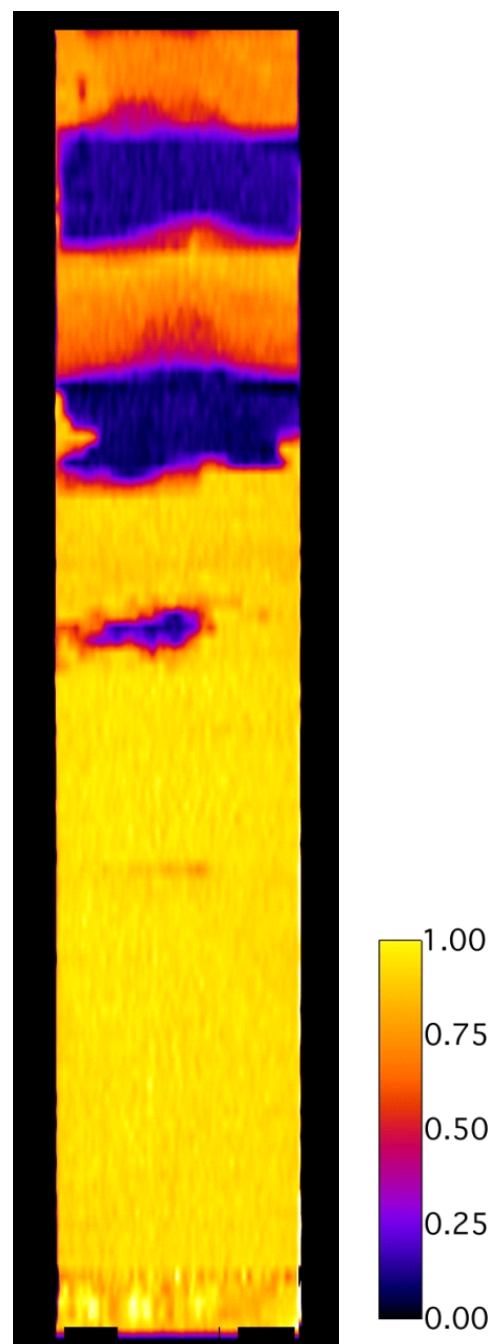

(b)

Fig. 60. (a) The porosity calculated from CT scans of the sand pack prior to hydrate formation. (b) The 'total saturation' of the sand pack after hydrate formation is complete, also calculated from CT scans. This is mathematically the saturation if only water and gas are present. This calculation is an intermediate step to calculating the hydrate saturation. What it shows clearly is greater hydrate saturation in the 'coarse' layers than in the 'fine' layers, for the top half of the column. For the bottom half of the column there does not appear to be a significant difference, though this will be investigated further.

\section{Fluid Phase Transport Model (During Hydrate Formation)}

\section{Initial condition}

An average gas saturation of $22 \%$ is initially placed into the sandpack before cooling starts. Based on the capillarity characteristics of the coarse and fine grained sections it is possible to estimate the layer-by-layer distribution of the overall $22 \%$ gas saturation. Figure 61 shows the gas saturation profile which satisfies the capillarity/gravity equilibrium along with the characteristic capillary curves of the fine and coarse sections. Based on the capillary curves it is expected that a large gas saturation, i.e. $S_{g}=0.9$, is established within the coarse layers and a much smaller gas saturation, i.e. $S_{g}=0.1$, within the fine grained layers. Based on the CT images 
of the sandpack at the end of experiment the bottom half of the sandpack is almost fully saturated with water. Therefore, the gas phase must have channeled through the bottom half of the sandpack establishing a very low gas saturation, e.g. $S_{g}=2 \%$, just enough to make a connected path of gas phase throughout the sandpack. An evidence of such connected path of gas throughout the sandpack is that the top and bottom pressure transducers essentially read the same pressure values during major part of the experiment. Note that the average gas saturation of the gas profile shown in Fig. 61 is also $22 \%$.

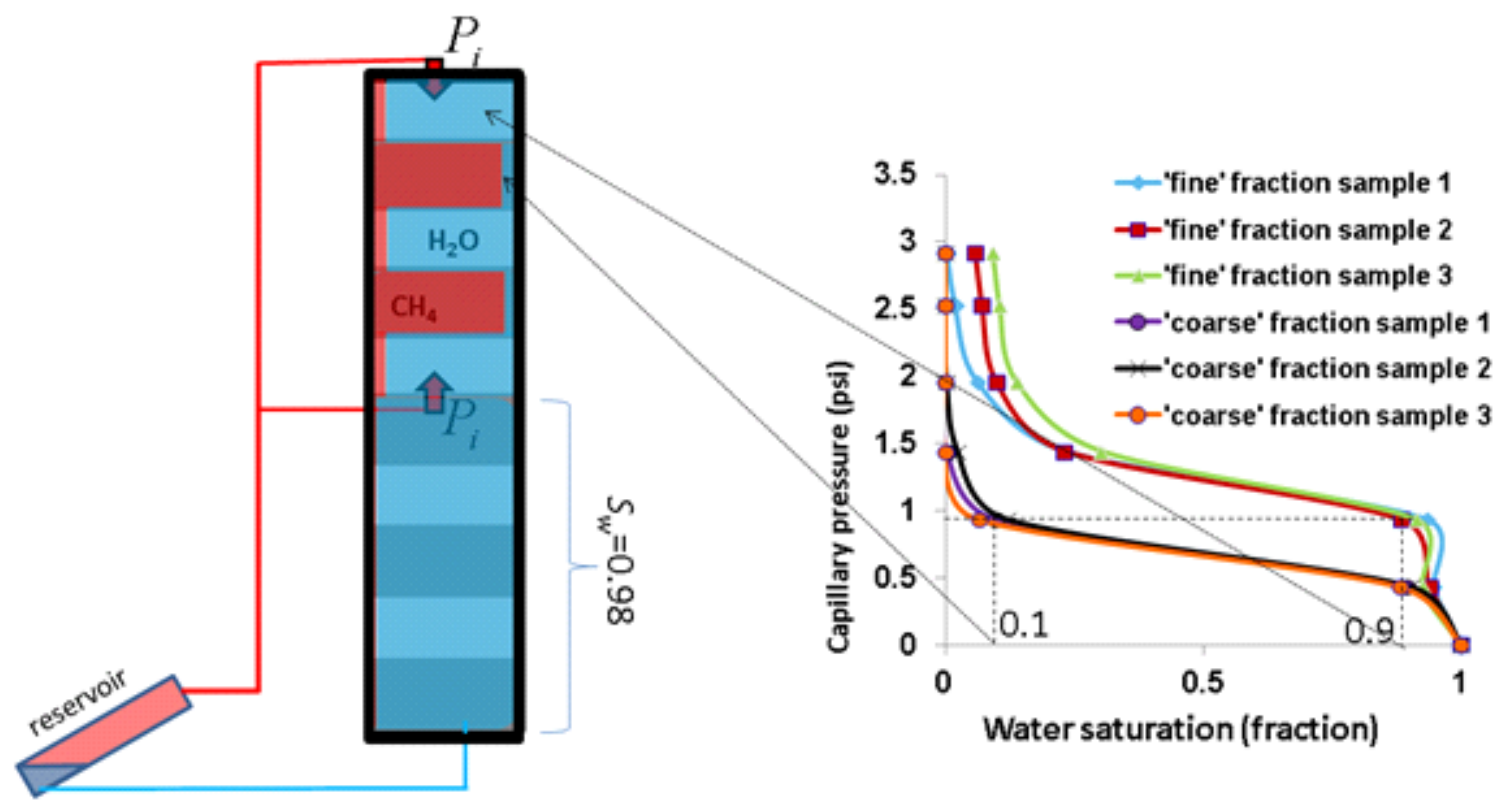

Fig. 61. The initial gas saturation profile within the sandpack determined from the characteristic capillary curves of the coarse and fine layered sections.

The graph of final hydrate saturation for the three initial gas saturations of interest here is shown in Fig. 62. As discussed in previous sections hydrate formation from gas and water requires flow of gaseous and aqueous phase into the GHSZ. The gaseous/aqueous phase proportion of the transported phase was shown to have a first order effect on the final hydrate saturation profile. 


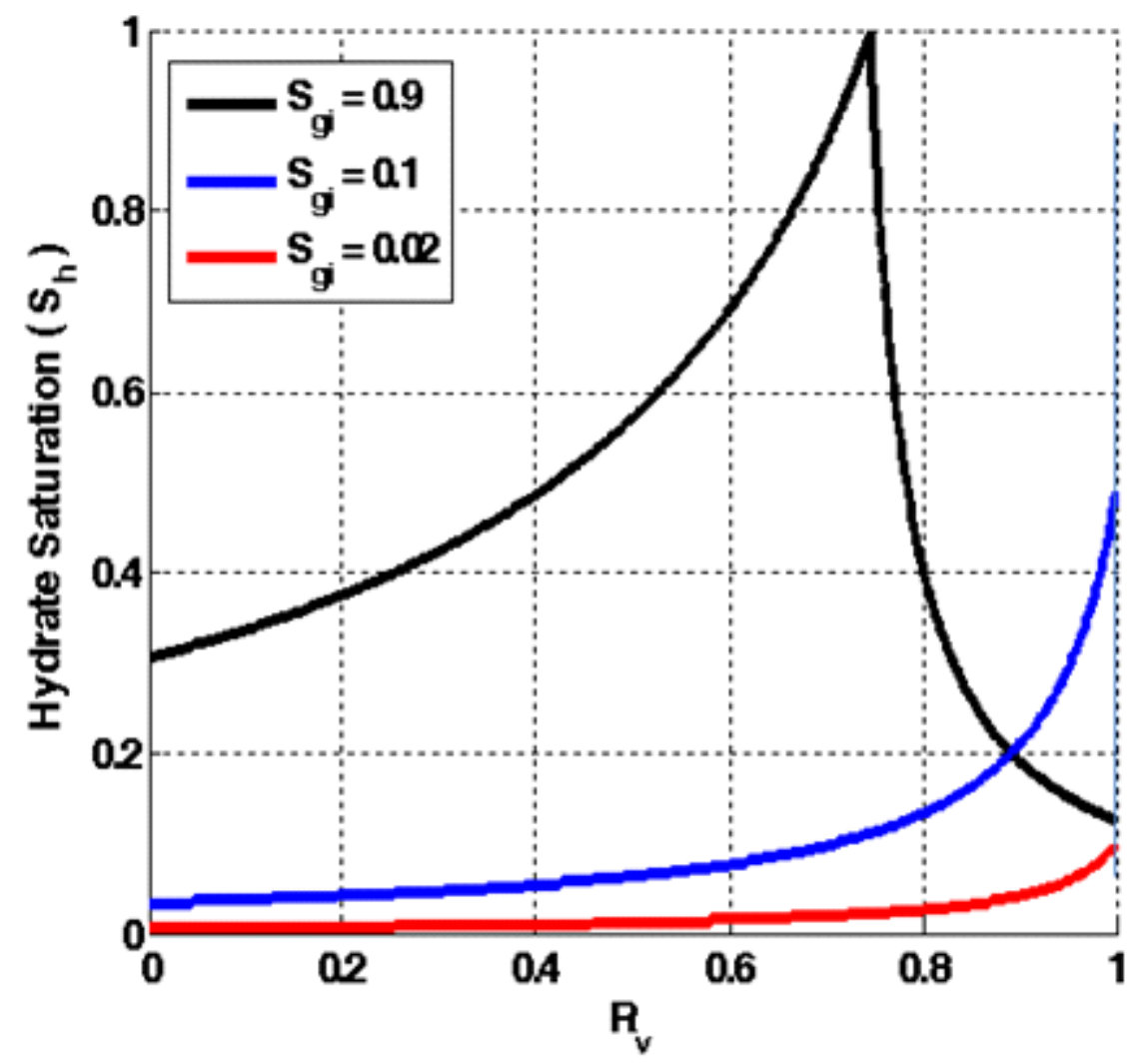

Fig. 62. Final hydrate saturation, $S_{h}$, versus gas volume fraction of the total phase fluxes, $R_{v}$, for the initial gas saturations in the layers shown in Fig. 61 . Methane density is $\sim 40 \mathrm{~kg} / \mathrm{m} 3$ at the operating temperature $\mathrm{T} \sim 5^{\circ} \mathrm{C}$ and average pressure of 750 psi.

\section{Formulation of the Driving Force}

Assume hydrate is forming in a portion of the sandpack containing an initial uniform gas saturation. We adopt a quasi-steady state method to estimate the gas/water fluxes transported into the hydrate-formation zone during hydrate formation. We denote the pressure (gas pressure) inside the hydrate-formation zone as $P_{j}$ (containing $n_{j}$ moles of gas), and the pressure of the gas elsewhere inside the system as $P_{i}$ (containing $n_{i}$ moles of gas). Obviously, before any hydrate is forming and the system is in equilibrium $P_{i}=P_{j}$; therefore, no flow is taking place when no hydrate is forming.

Assume that at time $t^{-}$when gas saturation is $S_{g}^{t^{-}}$(containing $n_{j}^{t^{-}}$moles of methane) and water saturation is $S_{w}^{t^{-}}$, a saturation increment of hydrate, $d S_{h}$, forms inside the hydrate formation zone. For this to happen, an incremental saturation of gas $d S_{g}^{u s e d}=\frac{\bar{V}_{g}}{\bar{V}_{h}} d S_{h}$ and an increment $d S_{w}^{\text {used }}=N \frac{\bar{V}_{w}}{\bar{V}_{h}} d S_{h}$ water were consumed. $\bar{V}_{g}, \bar{V}_{w}$, and $\bar{V}_{h}$ are molar volumes of 
methane, water and methane hydrate, respectively. The number of moles of gas inside the hydrate formation zone would also drop to $n_{j}^{t}=n_{j}^{t^{-}}-\frac{d S_{h}}{\bar{V}_{h}}$. The gas phase is assumed to expand and fill the void space instantly and thus $S_{g}^{t}=S_{g}^{t^{-}}+d S_{w}^{u s e d}-d S_{h}$. Therefore, the pressure at the hydrate formation zone would drop. Since the system pressure is controlled by the gas phase, the pressure at the hydrate-formation zone would drop to:

$$
P_{j}^{t}=P_{i}^{t}\left(\frac{S_{g}^{t^{-}}-d S_{g}^{u s e d}}{S_{g}^{t^{-}}+d S_{w}^{u s e d}-d S_{h}}\right)=P_{i}^{t}\left(\frac{S_{g}^{t^{-}}-d S_{g}^{u s e d}}{S_{g}^{t}}\right)
$$

assuming the ideal-gas law is valid.

The number of gas moles outside the hydrate formation zone and thus its pressure is assumed to remain unchanged, $n_{i}^{t}=n_{i}^{t^{-}}$and $P_{i}^{t}=P_{i}^{t^{-}}$, right before gas starts flowing to the hydrate formation zone.

$P_{j}^{t}<P_{i}^{t}$ implies a gas flow from outside the zone of hydrate-formation into the hydrate formation zone. Moreover, the aqueous phase would also experience this drop of pressure and would flow towards the zone of hydrate-formation. Flow of gas and water takes place until the pressure outside and inside of the hydrate-stability zone becomes equal again, i.e. $P_{i}=P_{j}$.

\section{Formulation of the Phase Fluxes}

We consider two cases for the mode of fluid transport. First we assume no capillarity-driven flow so that only pressure-driven viscous dominated flow occurs. We further assume that the saturation profile below the GHSZ does not change as hydrate forms and fluids flow to the GHSZ. We will show that in this case the proportion of gaseous phase flow, $R_{v}$, is not consistent with the large observed (inferred from CT image, Fig. 60 right panel) hydrate saturation within coarse layers. In the second case, we consider capillarity-driven flow together with pressuredriven flow, the former resulting from a capillary pressure gradient imposed by aqueous phase consumption during hydrate-formation. We will show that for the time-scale of this experiment the capillary-driven flow must have played a key role in forming large hydrate saturations from initial large gas saturations within the coarse layers as observed at the end of the experiment.

\section{Case 1: Viscous Dominated Flow}

Flow rate for water and gas is estimated from Darcy equation at each step. The increment in potential difference that each phase experiences would be $P_{i}-P_{j}$. Based on the apparatus setup, aqueous phase is fed only from below the hydrate formation zone while gas phase is supplied from the top and from the midpoint of the sandpack. The water flow rate in the infinitesimal time interval $[t, t+d t]$ is assumed constant, $q_{w}=\frac{k k_{r w}}{\mu_{w}} A \frac{P_{i}^{t}-P_{j}^{t}}{\frac{L}{2}+L_{2}}$, where $L$ is the length of the sandpack. Similarly, gas flow rate in the time interval $[t, t+d t]$ would be: 
$q_{g}=\frac{k_{1} k_{r g}}{\mu_{g}} A \frac{P_{i}^{t}-P_{j}^{t}}{L_{1}}+\frac{k_{2} k_{r g}}{\mu_{g}} A \frac{P_{i}^{t}-P_{j}^{t}}{L_{2}}$

Using the above gas and aqueous phases flow rates, $P_{i}^{t+d t}$ is calculated as:

$$
P_{i}^{t+d t}=P_{i}^{t}\left(1-\frac{\frac{q_{g} d t}{\bar{V}_{g}}}{n_{i}^{t}}\right)
$$

Since gas and water both would flow towards the hydrate formation zone, and since water is the incompressible phase, $S_{g}^{t+d t}$ would decrease from $S_{g}^{t}$ by the amount of water (pore volumes) that is supplied to the hydrate formation zone, i.e. $S_{g}^{t+d t}=S_{g}^{t}-q_{w} d t / P V_{H S Z}$, wherein $P V_{H S Z}$ denotes the pore volume inside the hydrate formation zone. In fact, both water flow and the moles of gas transported, $q_{g} d t / \bar{V}_{g}$, into the hydrate formation zone contribute to increasing $P_{j}$ (while $P_{i}$ is decreasing due to loss of moles of gas). Therefore, $P_{j}^{t+d t}$ is calculated as in Eq. (96).

$$
P_{j}^{t+d t}=P_{j}^{t}\left(\frac{\frac{n_{j}^{t}+\frac{q_{g} d t}{\bar{V}_{g}}}{S_{g}^{t+d t}}}{\frac{n_{j}^{t}}{S_{g}^{t}}}\right)=P_{j}^{t}\left(\frac{S_{g}^{t}}{S_{g}^{t+d t}}\right)\left(\frac{n_{j}^{t+d t}}{n_{j}^{t}}\right)
$$

Note that, this increase in $P_{j}$ and the corresponding decrease in $P_{i}$ continues until $P_{i}=P_{j}$ and the gas/water flow stops. This analysis is carried forward through incremental time steps, $d t$, and the system approaches an equilibrium state until another increment of hydrate $d S_{h}$ forms and the above can be applied similarly.

\section{Application of Pressure-Driven Viscous Flow Model to Stage 2 of Experiment}

To illustrate the application of the above model, we analyze the hydrate formation within the coarse layer when the BGHSZ is at depth 8.1 " from the top of the sandpack. Since we only consider the pressure-driven viscous dominated flow, $R_{v}$ is basically governed by the ratio of phase mobilities, $\frac{\lambda_{g, e f f}}{\lambda_{w, e f f^{+}} \lambda_{g \text { ef }}}$. Note that since we have two inlets for the gas phase and only one inlet for water from below, $R_{v}$ is slightly greater than $\frac{\lambda_{g, e f f}}{\lambda_{w, e f f}+\lambda_{g \text { ef }}}$. 
Inferred data from the experiment corresponding to this step is presented in Fig. 63. The number of moles of methane consumed was inferred from the pressure change in the system (which was measured by two pressure transducers, one at the top and one at the bottom). The following table summarizes the amount of methane that was used and the resulting amount of methane hydrate. Density of water was assumed to be $\rho_{w}=1000 \mathrm{~kg} / \mathrm{m}^{3}$, density of methane was assumed to be $\rho_{h}=914 \mathrm{~kg} / \mathrm{m}^{3}$ (Sloan, 1998) and the density of methane at $\mathrm{P}=788 \mathrm{psig}$ (system pressure right before hydrate formation starts) was calculated to be $\rho_{g} \approx 44 \mathrm{~kg} / \mathrm{m}^{3}$. During the analysis as the pressure of the gas is decreasing, the density value and thus the methane molar volume is updated. Molar volumes of water, hydrate and methane at $\mathrm{P}=788 \mathrm{psig}$ and $\mathrm{T}=5^{\circ} \mathrm{C}$ are reported in Table 5.
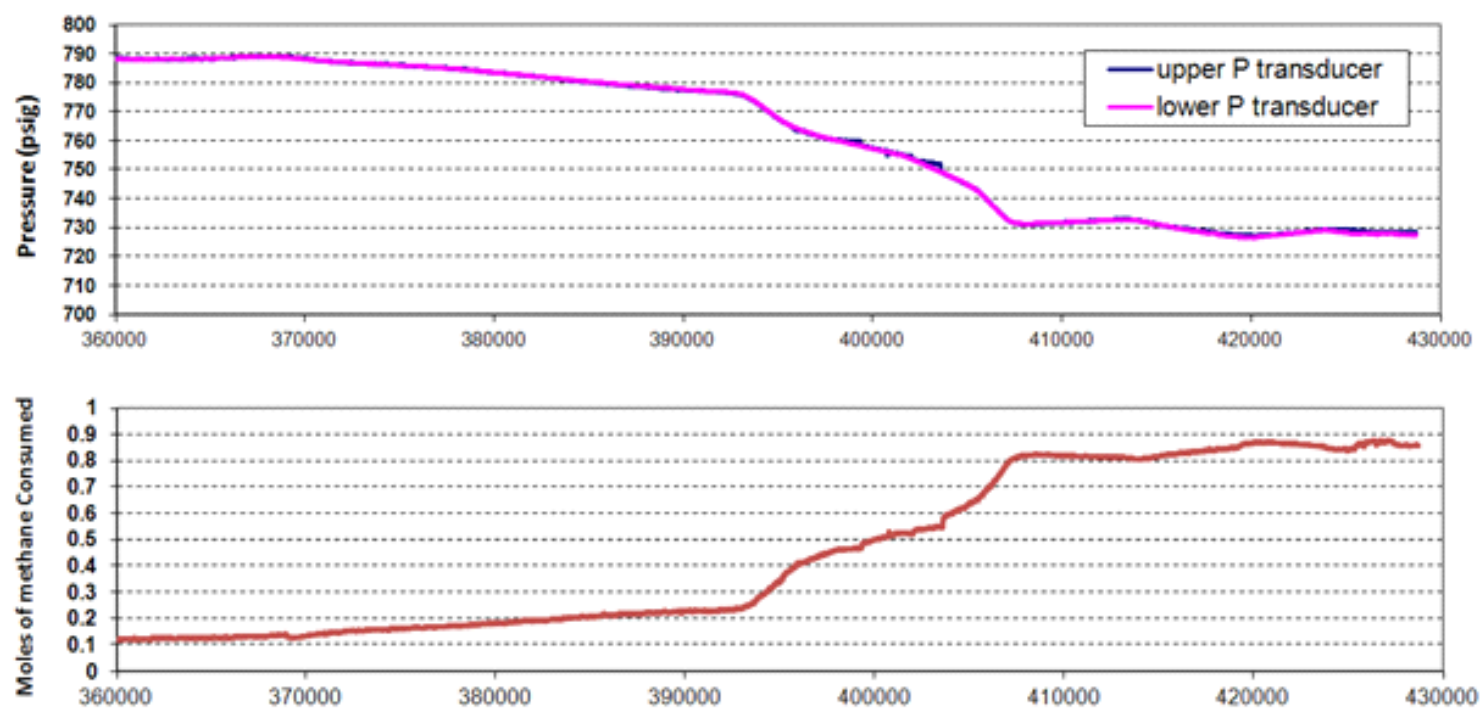

Fig. 63. Experiment results: BGHSZ descends from $z=0$ to $z=20.6 \mathrm{~cm}$ and stays at $20.6 \mathrm{~cm}$ for about 24 hours. Horizontal axis shows time in seconds. The moles of methane consumed (bottom panel) are computed from the pressure in the reservoir (top panel) and the mass of water in the reservoir (not shown).

Table 5. Experiment Data: Hydrate is Assumed to Form Within the Height Interval $[0,20.6] \mathrm{cm}$ of the Sandpack

\begin{tabular}{c|c|c|c|c|c|c}
\hline \multicolumn{3}{c|}{$\begin{array}{c}\text { Molar volumes } \\
(\mathrm{m} 3 / \mathrm{kmole})\end{array}$} & \multicolumn{2}{c}{$\begin{array}{c}\text { HSZ pore } \\
\text { volume }\end{array}$} & \multicolumn{3}{|c}{$\begin{array}{c}\text { Inferred information from experimental data } \\
\bar{V}_{w}\end{array}$} & $\bar{V}_{g}$ & $\bar{V}_{h}$ & $P V_{H S Z}\left(\mathrm{~cm}^{3}\right)$ & $\begin{array}{c}\text { Moles of } \mathrm{CH}_{4} \\
\text { converted (moles) }\end{array}$ & $\begin{array}{c}\text { Average Hydrate } \\
\text { Saturation, } S_{h}\end{array}$ & $\begin{array}{c}\text { Water Entered } \\
\text { Sandpack (g) }\end{array}$ \\
\hline 0.018 & 0.363 & 0.136 & 336.4 & $\approx 0.8$ & $\approx 33 \%$ & $\approx 12$ \\
\hline
\end{tabular}

We impose the hydrate-formation rate inferred from the experiment data as a boundary condition on the model. The goal in doing this is to determine from the model how the fluids phases will move in response to this condition. An average hydrate saturation of $S_{h}=0.33$ was formed in the GHSZ in the time interval [3.6 4.2] $\times 10^{5} \mathrm{sec}$; therefore, an average rate of hydrate formation (sat unit/sec) may be extracted. However, based on the graph of consumed moles of methane versus time (Fig. 63) it is clear that there are three different major rates of hydrate formation over the course of time within the interval [3.6 4.2] $\times 10^{5} \mathrm{sec}$ : slow between $3.6 \times 10^{5} \mathrm{~s}$ and $3.9 \times 10^{5} \mathrm{~s}$, fast between $3.9 \times 10^{5} \mathrm{~s}$ and $4.08 \times 10^{5} \mathrm{~s}$, and slow again for the remainder of the 
interval. Therefore, in the modeling the rate of hydrate formation appropriate to each time interval was imposed. An intrinsic permeability of 10 Darcy was assumed for the sandpack and the relative permeability shown in Fig. 64 was used.
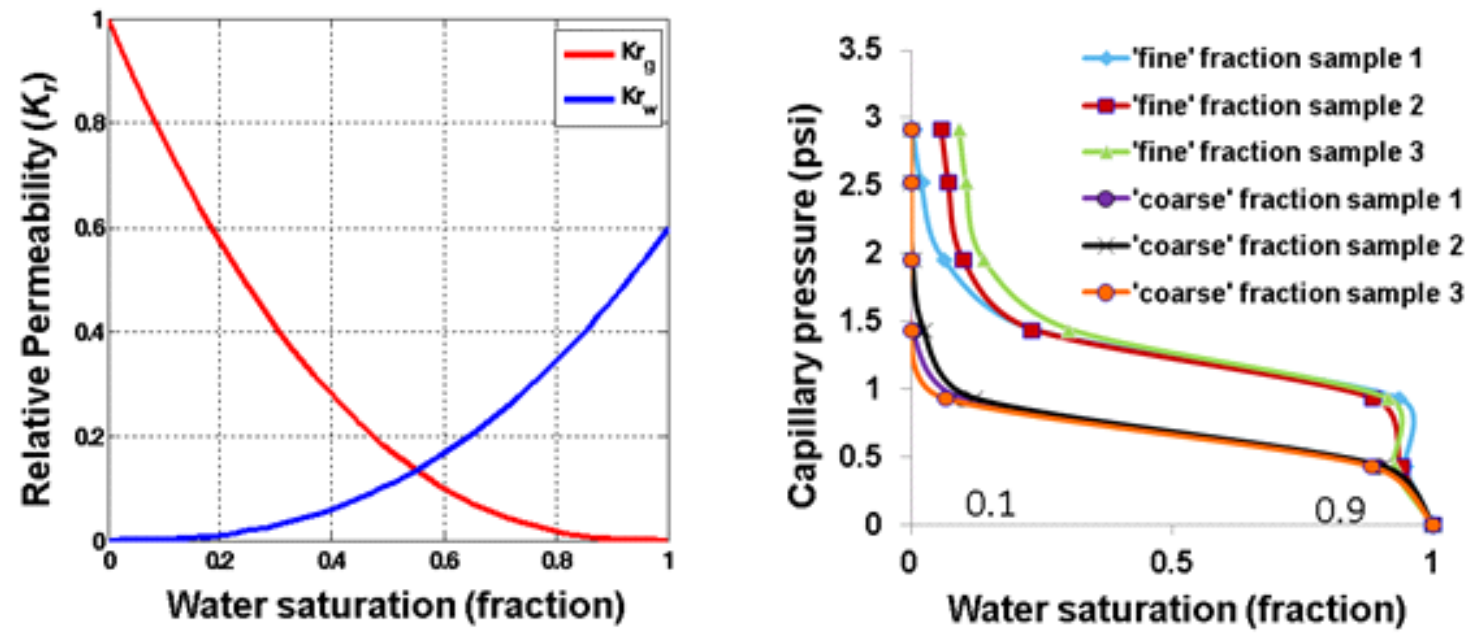

Fig. 64. Left: Assumed characteristic relative permeability curves for both coarse and fine layers; Right: Experimental capillary curves of fine and coarse layers based on porous plate method.

The effective mobilities used for flow into the top coarse layer, shown in Fig. 65, are calculated using the harmonic average the mobility of the layers below the coarse layer as follows:

$$
\begin{aligned}
& \lambda_{w, \text { ef } f}=\frac{0.8}{\frac{0.5}{\left.\lambda_{w}\right|_{S_{g}=0.02}}+\frac{0.2}{\left.\lambda_{w}\right|_{S_{g}=0.1}}+\frac{0.1}{\left.\lambda_{w}\right|_{S_{g}=0.9}}} \\
& \lambda_{g, \text { ef } f}=\frac{0.3}{\frac{0.2}{\left.\lambda_{g}\right|_{S_{g}=0.1}+\frac{0.1}{\left.\lambda_{g}\right|_{S_{g}=0.9}}}}
\end{aligned}
$$

There will be another effective mobility calculated for gas flow from above which can be calculated similar to Eq. (98). The effective mobilities of gaseous and aqueous phase flowing from below are $\lambda_{g, e f f}=394.5$ and $\lambda_{w, e f f}=8.7$; consequently $R_{v}$ is almost 1 . 


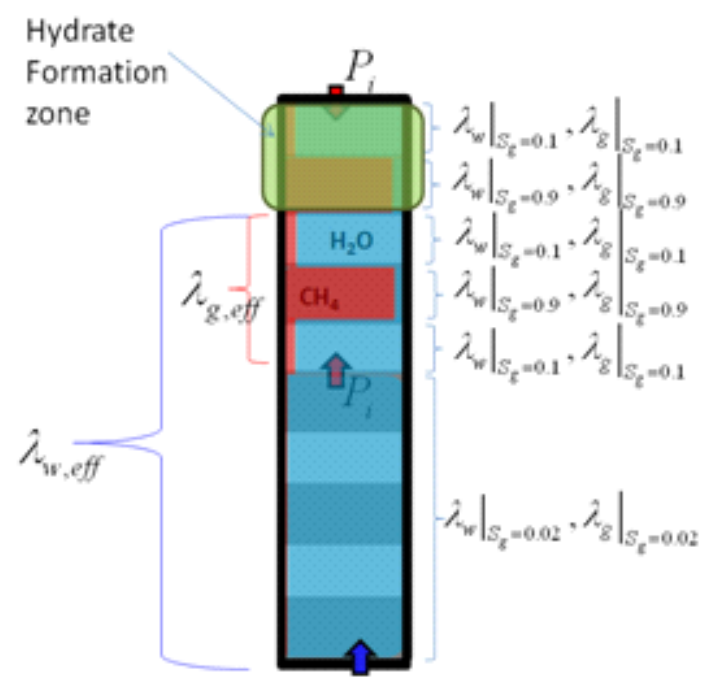

Fig. 65. Illustration of the effective mobilities used in the viscous dominated flow calculations.

Using these mobilities, the model predicts that hydrate formation stops at about $13 \%$ hydrate saturation, (Fig. 66), far less than the observed average value of 33\% (Table 3). This limited hydrate saturation of $13 \%$ is what we would expect from Fig. 62 and $R_{v}$ nearly unity. In effect the GHSZ runs out of aqueous phase because the water phase mobility is so much smaller than the gas phase mobility. Note that the model predicts that the remaining pore space in the layer would be filled with gas, not water. However, based on the final CT-scan images a large hydrate saturation of about $80 \%-90 \%$ was established within the top coarse layer sections. This indicates that viscous dominated flow cannot explain the experimental observations. 

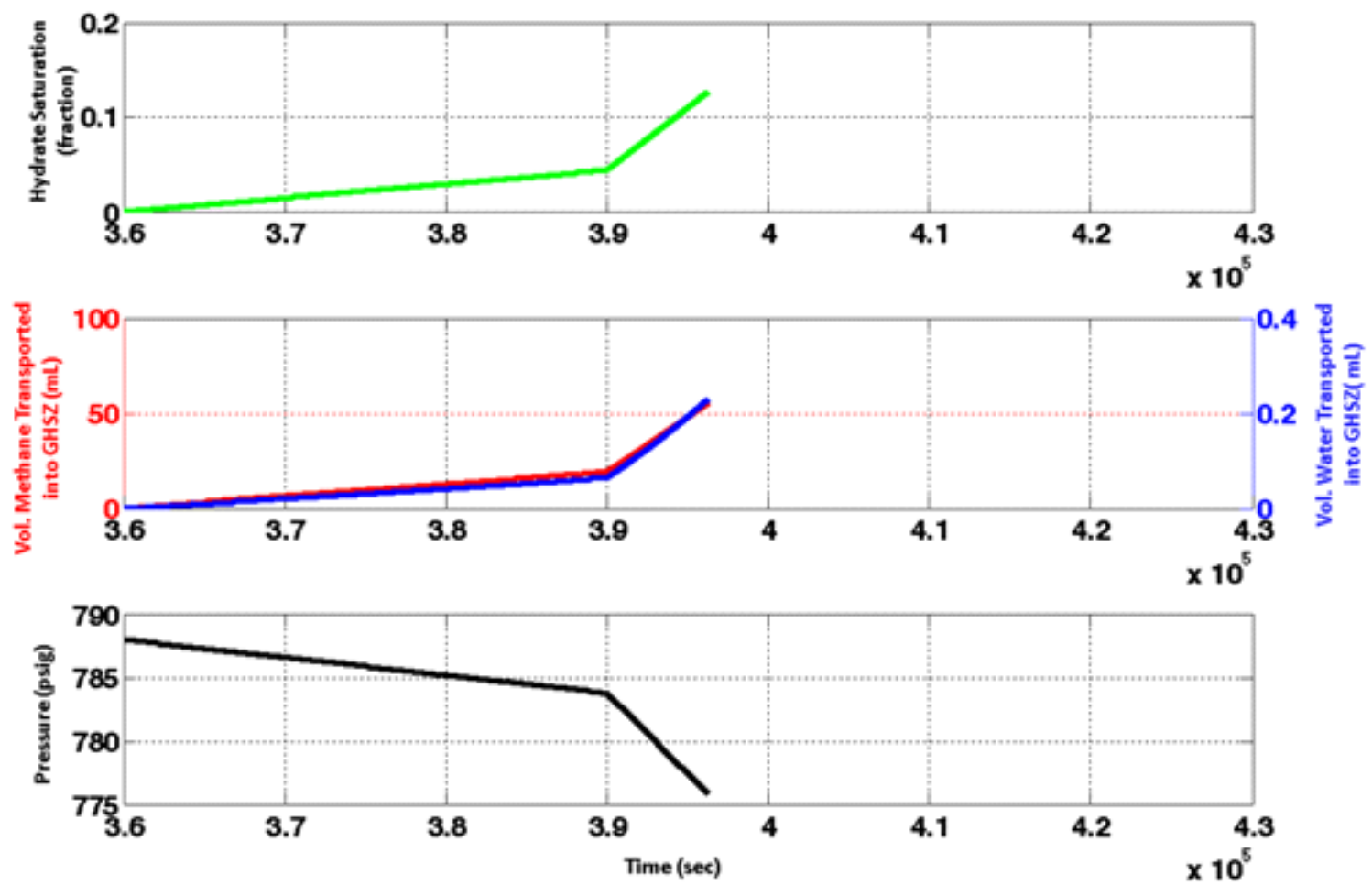

Fig. 66. Simulation results for hydrate formation within the coarse layer between $[9.4 \mathrm{~cm} 20.6 \mathrm{~cm}]$ when only viscous dominated flow is taken into account. BGHSZ descends from $z=0$ to $z=20.6 \mathrm{~cm}$ and stays at $20.6 \mathrm{~cm}$ for about 24 hours. Rate of hydrate formation determined from Fig. 63 was imposed on the model as a boundary condition.

\section{Case 2: Pressure-Driven + Capillarity-Driven Flow}

This case accounts for another contribution to aqueous phase flow towards the GHSZ, the flow due to a capillary-pressure gradient imposed by aqueous phase consumption during hydrate formation. Similar to the discussion above, when a saturation increment of hydrate, $d S_{h}$, forms inside the GHSZ, $d S_{g}^{u s e d}=\frac{\bar{V}_{g}}{\bar{V}_{h}} d S_{h}$ saturation of gas and $d S_{w}^{u s e d}=N \frac{\bar{V}_{w}}{\bar{V}_{h}} d S_{h}$ saturation of water were used to form the latter increment of hydrate. The gas phase is assumed to expand and fill the void space instantly and thus $S_{g}^{t}=S_{g}^{t^{-}}+d S_{w}^{u s e d}-d S_{h}$. The water saturation decreases to $S_{w}^{t}=S_{w}^{t^{-}}-d S_{w}^{u s e d}$ at the same moment. This reduction in water saturation would be equivalent to an increase of the capillary pressure from the initial capillary pressure, i.e. equilibrium state. The capillarity contribution to water flow is:

$u_{w, c a p}=k \frac{\lambda_{w} \lambda_{g}}{\lambda_{w}+\lambda_{g}}\left(\frac{\partial P_{c}}{\partial z}+\left(\rho_{w}-\rho_{g}\right) g\right)$

Note that in the above equation downward direction is the + direction. When the system is in equilibrium we have $-\frac{\partial P_{c}}{\partial z}=\left(\rho_{w}-\rho_{g}\right) g$; therefore, $u_{w, c a p}=0$. When an increase in capillary 
pressure takes place due to a reduction in aqueous phase saturation $-\frac{\partial P_{c}}{\partial z}>\left(\rho_{w}-\rho_{g}\right) g$ and thus a nonzero flux of water flows towards the GHSZ due to a gradient in capillary pressure.

Figure 67 shows the modeling results when we account for the capillary-driven flows. A clear increase in magnitude of water flowing into the GHSZ is observed in Fig. 67 compared to that in Fig. 66. This substantial increase in the amount of water flux is due to the capillaritydriven flow. At the time, $t=420,000 \mathrm{sec}$ when $S_{h}=33 \%$, the gaseous and aqueous phase saturations are $S_{g}=58.54 \%$ and $S_{w}=8.46 \%$, respectively. Note that the initial gaseous and water saturations were $S_{g, i}=90 \%$ and $S_{w, i}=10 \%$, and thus it is clear that gaseous phase saturation is decreasing much faster than that of aqueous phase. In fact, capillary-dominated flow is crucially contributing toward maintaining the water saturation to make formation of large hydrate saturations possible.
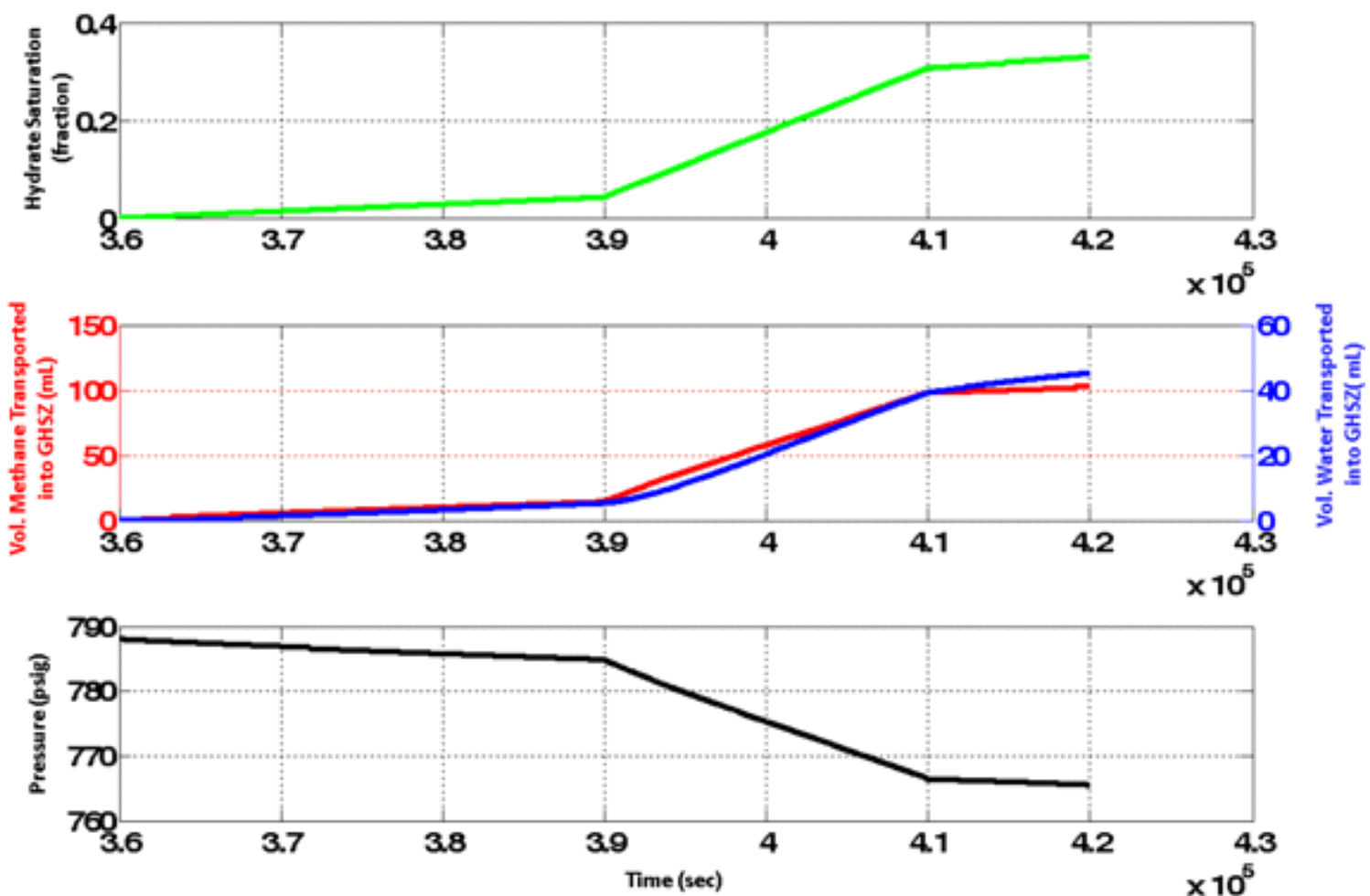

Fig. 67. Simulation results for hydrate formation within the coarse layer between $[9.4 \mathrm{~cm} 20.6 \mathrm{~cm}] \mathrm{when}$ both viscous dominated and capillarity-driven flows are taken into account. BGHSZ descends from $z=0$ to $z=20.6 \mathrm{~cm}$ and stays at $20.6 \mathrm{~cm}$ for about 24 hours. Rate of hydrate formation was adopted from the experiment.

\section{Is Hydrate Formation Controlled by the Rate of Fluid Transport?}

The preceding analysis shows that capillarity-driven movement of water enables flow at rates sufficient to supply the observed rate of hydrate formation. Moreover the model indicates that neglecting capillarity-driven movement causes the GHSZ to be water-starved. This is a limiting case as very large saturation gradients would obviously arise long before the water saturation in the layer were completely consumed. This suggests that even if the flow were pressure-driven (viscous dominated) for some period of time, the system would evolve to a state in which 
capillarity-driven flow must occur. These considerations raise an interesting speculation: the rate at which hydrate can be formed from a gas accumulation is ultimately limited by the rate at which the aqueous phase can be supplied to the GHSZ.

The experiment provides some support for this speculation. The images on the following pages show the moles of gas converted into methane hydrate in each step of descent of the BGHSZ as well as the mass of water in the reservoir versus time. In the first three steps, methane is consumed intermittently at large rates $\left(>3 \times 10^{-5} \mathrm{gmol} / \mathrm{s}\right)$, while the net amount of water sucked into the sandpack from the reservoir is small. In fact the mass of water in the reservoir fluctuates rather wildly in steps 2 through 4 . In fact sometimes water enters the reservoir from the sandpack. The model shows that large rates of hydrate formation cannot be sustained with only viscous flow. The fluctuations in the water flux between reservoir and sandpack may be a manifestation of this limit, as the system may have become starved of water in various locations during the intervals of rapid hydrate formation. The starvation would have caused hydrate formation to stop, halting the requirement for influx of fluids. Meanwhile local capillarity-driven flow restored water in other locations, enabling hydrate formation to resume.

A transition from viscous-dominated to flow with a significant contribution from capillarity seems to have occurred after step 4. Unlike steps 1 through 4, the behavior does not change qualitatively during steps 5 through 8 . The rate of hydrate formation is steady, decreasing slowly with time and accompanied by a steady draining of water from the reservoir into column. The rate of hydrate formation is much smaller throughout this period than it was during the largerate-of-formation intervals in steps 2 and 3. The small rate was not for lack of gas to be converted; at the end of step 4, the cumulative gas converted was just $64 \%$ of the value at the end of the experiment. Nor is the small rate due to lack of hydrate stability: the BGHSZ is far below the upper portion of the column where the hydrate is forming, and no salinity builds up during hydrate formation because the aqueous phase is distilled water. The simplest explanation for the late-stage behavior is that the system has settled into a balanced state. The rate of hydrate formation is just large enough to impose pressure and capillary pressure gradients that in turn are just large enough to induce the rates of gas and water transport needed to support hydrate formation. Since even the smaller rate of hydrate formation cannot be supplied only by pressuredriven viscous flow, this suggests that capillarity-driven flow of water is essential to the formation of hydrate. Moreover, the rate of capillarity-driven flow determines the rate of hydrate formation. 
Rate of Hydrate formation $=5 \times 10^{-6}$ (moles hydrate/second)
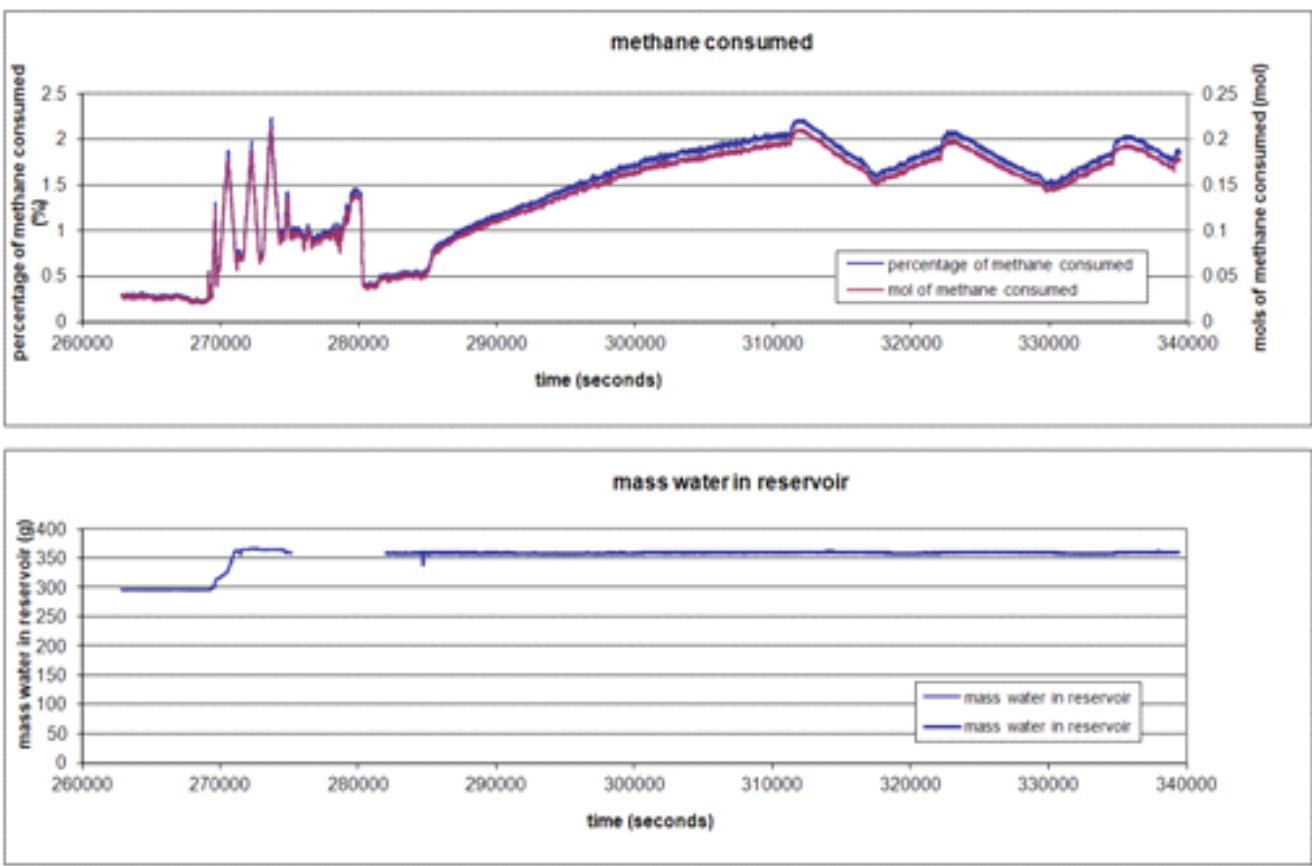

Rate of Hydrate formation =Shown on Graph
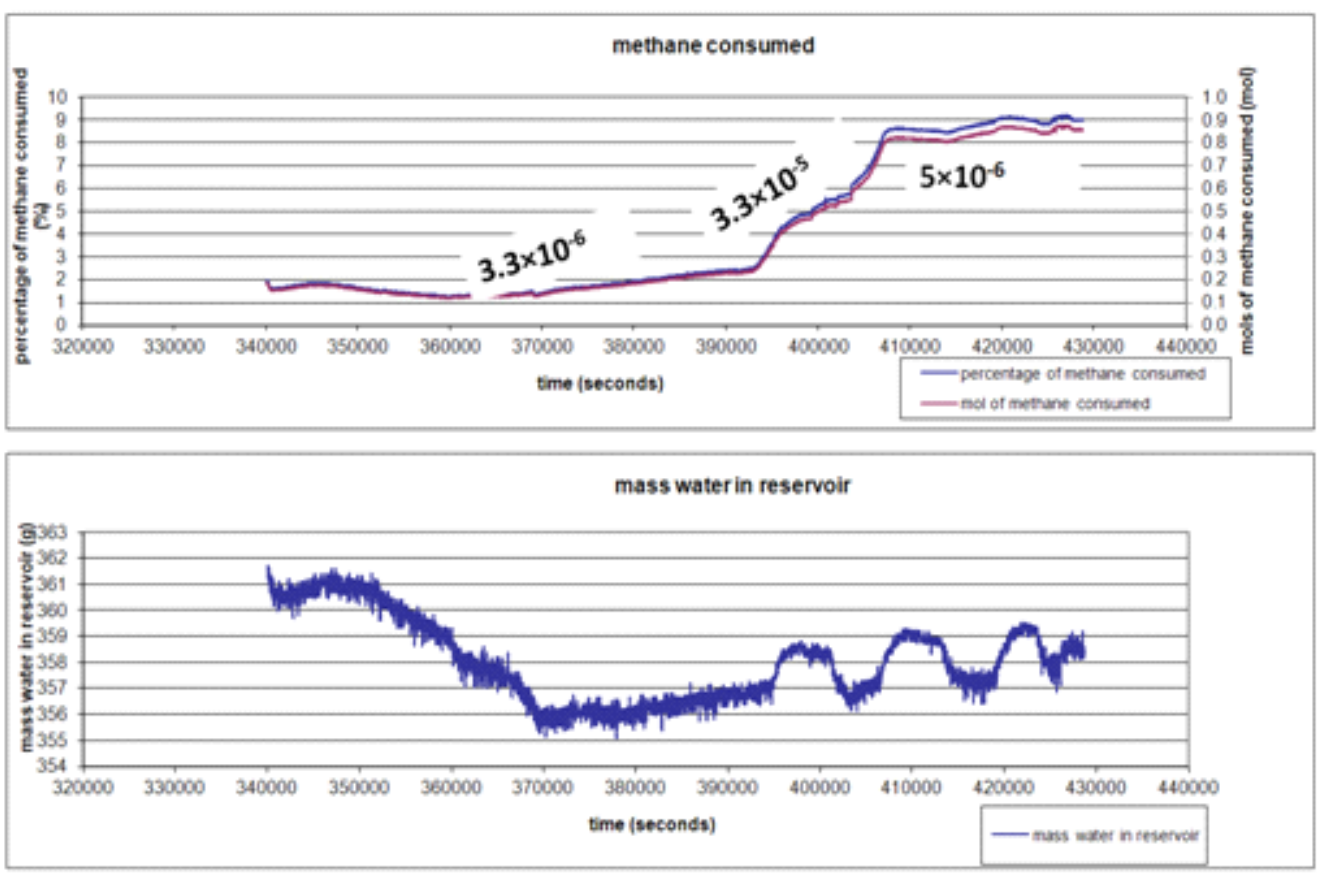

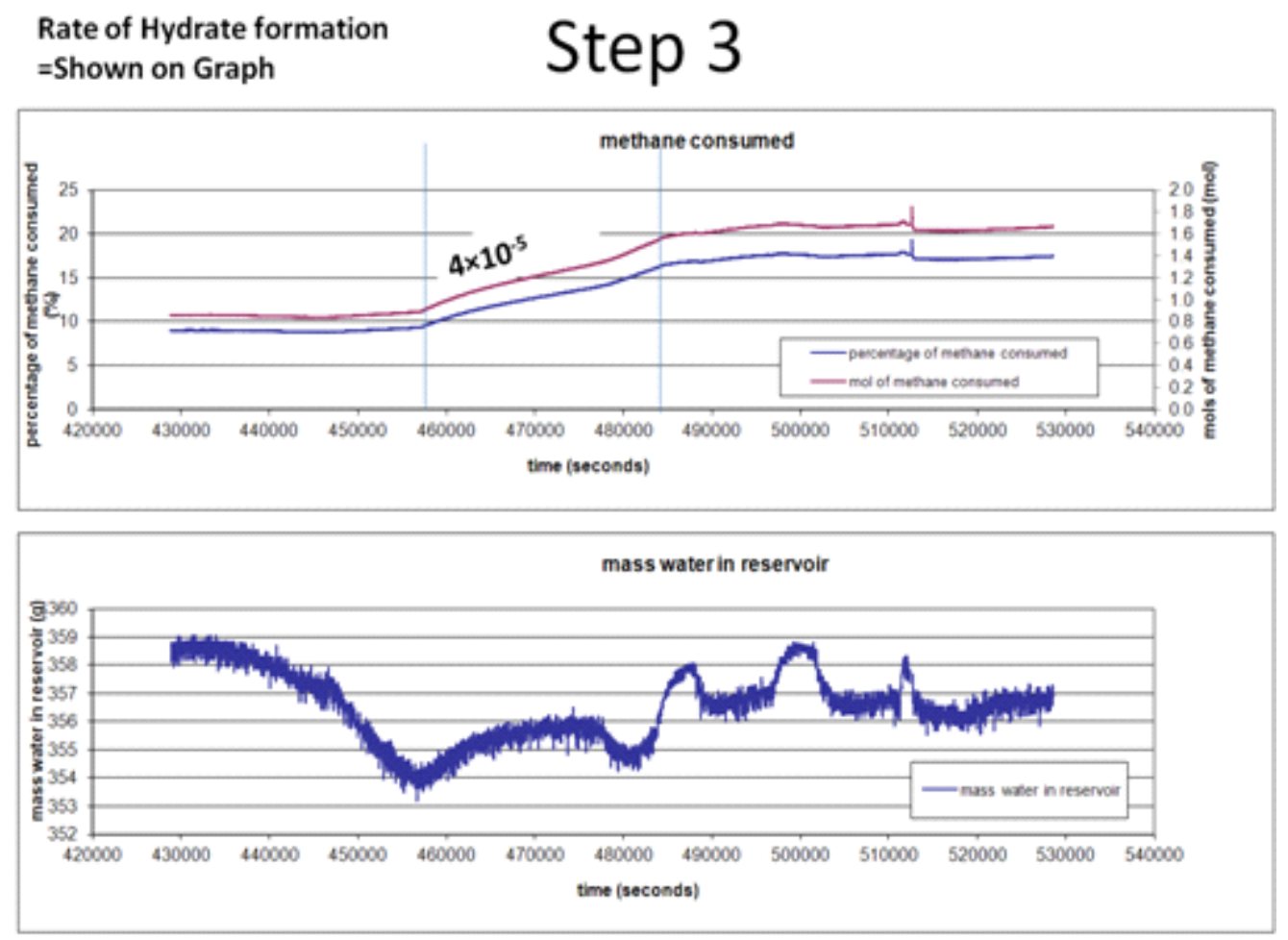
Rate of Hydrate formation
$=3.6 \times 10^{-6}$ (moles hydrate/second) Step 4
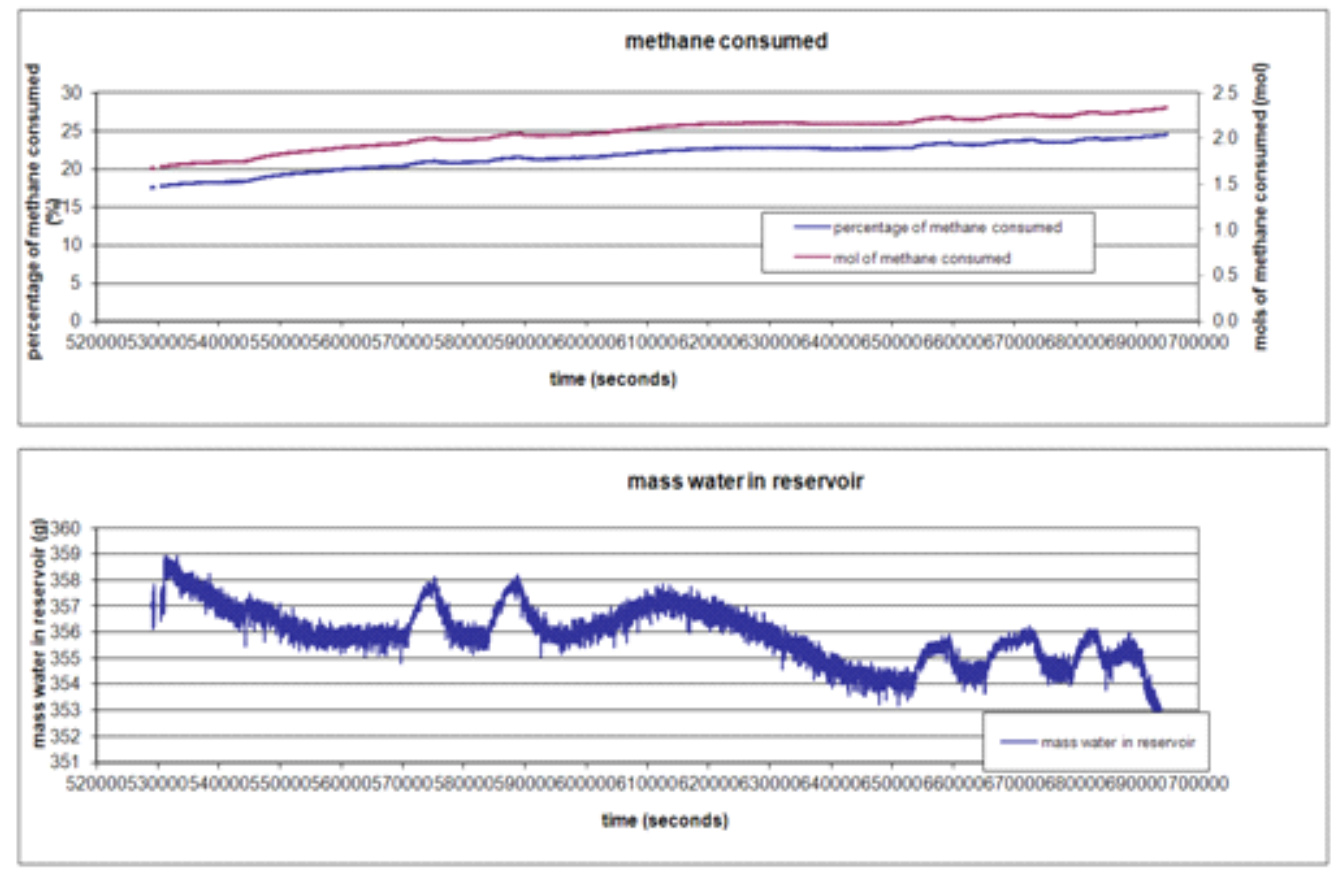